International Scientific Organization

http://iscientific.org/

Chemistry International

www.bosaljournals.com/chemint/

\title{
Bioassays based on higher plants as excellent dosimeters for ecotoxicity monitoring: A review
}

\author{
Munawar Iqbal1 ${ }^{1,}$, Mazhar Abbas' ${ }^{2}$, Jan Nisar ${ }^{3}$, Arif Nazir ${ }^{1}$ and Ahmad Zaman Qamar ${ }^{4}$ \\ 1Department of Chemistry, The University of Lahore, Lahore, Pakistan \\ ${ }^{2}$ Jhang-Campus, University of Veterinary \& Animal Sciences Lahore, Pakistan \\ ${ }^{3}$ National Centre of Excellence in Physical Chemistry, University of Peshawar, Peshawar 25120, Pakistan \\ Department of Chemistry and Biochemistry, Southern Illinois University, Carbondale, Illinois, United States \\ *Corresponding author's E. mail: bosalvee@yahoo.com
}

\section{A R T I C L E I N F O}

Article type:

Review article

Article history:

Received January 2017

Accepted June 2018

January 2019 Issue

Keywords:

Industrial waste

Contaminated soil

River water

Allium cepa

Cytotoxicity

Genotoxicity

Mutagenicity

\section{A B S T R A C T}

All sorts of pollution on the planetcan be traced back to development of industries and the most important among them is water pollution. Clean technologies, management practices and regular monitoring of effluents could be helpful to minimize the contamination of watersheds. Allium cepa (A. cepa) has been recognized as a promising genetic model to detect the toxicity of industrial wastewater, contaminated soil, river water, nuclear contamination and even for those systems which are considered non toxic. A. cepa is distinguished as a low cost test, easy to handle and sensitive to both in vivo and in vitro models. It offers the detection of damages in genetic material quantitatively and the results can be generalized for other biological and ecological systems. Moreover, the pollutants can be classified on the basis of this test present in industrial effluents and their mechanism of action on genetic material. This review focuses on the studies undertaken to evaluate the toxicity of industrial wastewater, contaminated river and soils.

(c) 2019 International Scientific Organization: All rights reserved.

Capsule Summary: The applications of bioassays for ecotoxicity monitoring are regarded as excellent technique and Allium cepa was exemplified for ecotoxicity monitoring of variety of systems.

Cite This Article As: M. Iqbal, M. Abbas, J. Nisar, A. Nazir and A. Z. Qamar. Bioassays based on higher plants as excellent dosimeters for ecotoxicity monitoring: A review. Chemistry International 5(1) (2019) 1-80.

\section{INTRODUCTION}

From last few decades, there is a growing concern of pollution, which not only affect the animal and plant, but also human beings as well. The water bodies serve as temporary or final receptors of diverse variety of contaminants, which consequently contaminate the entire watershed and adjoining territories (Barbério et al., 2009; Bianchi et al., 2011; Ghodake et al., 2011; Kumari et al., 2011; Salles et al.,
2016). The rapid stride in industrialization is major concern and at present is a severe threat to the survival of living organisms and jeopardizing the ecological balance (Abbas et al., 2015; Iqbal et al., 2013a; Iqbal, 2016; Iqbal et al., 2016b; Islam et al., 2015; Manzoor et al., 2013; Mushtaq et al., 2016; Nadeem et al., 2016a; Rashid et al., 2016; Tabrez and Ahmad, 2011; Ullah et al., 2013; Younas et al., 2015).

Among the damages caused by chemical agents to exposed organisms, genotoxic and mutagenic effects have shown to be worrying, which can lead to several health 
problems and also affect future generations due to inheritable alterations in genetic material (Leme and MarinMorales, 2009). Genetic toxicology is involved in detecting compounds capable of causing DNA damage with the aim of understanding potential biological consequences and molecular mechanisms of genetic material. Mutagens screening in environmental samples using in vivo method is reliable to measure the extent of pollution load and this biological assessment is compulsory for effluent discharge from industries before being mixed with watersheds and adjoining territories (Akinboro et al., 2011; de Souza Pohren et al., 2013; Hemachandra and Pathiratne, 2015; Kannangara and Pathiratne, 2015b; Rodrigues et al., 2010).

The biological tests, especially short-term bioassays on bacteria or higher plants is the best way to detect and estimate the pollution load in any matrices approach (Gana et al., 2008; Mesi and Kopliku, 2012). Many plant species have a wide range of applications as indicators of cytogenetic and mutagenic effects of environmental physical and chemical polluting agents (Çavuşoğlu et al., 2011; Juchimiuk and Maluszynska, 2005).

The cytogenetic tests in plants are relatively inexpensive and can easily be handled and have shown good correlation with other bio-testing systems (Fiskesjo, 1988) and is also a rapid and sensitive method for environment monitoring. The plants like Vicia faba, Zea mays, Tradescantia, Nicotiana tabacum, Crepis capillaries, Pisum sativum, Hordeum vulgare and Allium cepa (A. cepa) have been used for this purpose (Akintonwa et al., 2009; Andrade et al., 2008; Bagatini et al., 2009; Chandra et al., 2005; do Canto et al., 2013; Fatima and Ahmad, 2006b; Goncharuk et al., 2011; Iqbal et al., 2015a; Iqbal and Bhatti, 2014, 2015a; Iqbal et al., 2014; Iqbal and Nisar, 2015; Srivastava et al., 2005).

\section{Advantages of $A$. cepa test versus other biological assays}

A. сера characteristics make it an excellent genetic model to assess environmental pollutants and can be used for monitoring chemical and physical toxic agents. The application of $A$. cepa is not only due to the sensitivity to detect mutagens, but also to the possibility of assessing several genetic endpoints ranges from point mutation to chromosome aberrations (CA) in meristematc cells as well as $\mathrm{F}_{1}$ generation (Leme and Marin-Morales, 2009; Paz et al., 2006). First of all Leven (Levan, 1938) introduced the A. cepa test for mitotic study since that time it is being used frequently. The $A$. cepa test is considered an excellent presentative of in vivo biological test, where the roots grow in direct contact with the substance of interest and enabling to detect the possible damage to the DNA and results can be generalized for divsrse animal and plant biodiversity as a model. Additionally, the structural aberration and numerical chromosomal alterations can be directly visualized. Moreover, A. cepa is distinctive regarding its efficiency as compared to other bioassays, good correlation with other test-systems, easy handling, low-cost and have ideal size as well as chromosome number $(2 \mathrm{n}=16)$. $A$. cepa can be used for the indication of toxic compounds (mutatoxic, cytotoxic and genotoxic etc) and due to its kinetic characteristics of proliferation as compared to Vicia faba, Zea mays, Tradescantia, Nicotiana tabacum, Crepis capillaries, Pisum sativum and Hordeum vulgare, is more suitable for chromosomes and nuclear study (Leme et al., 2008; Leme and Marin-Morales, 2009; Magdaleno et al., 2008; Rank, 2003; Smaka-Kincl et al., 1996; Souza et al., 2009).

Furthermore, cytogenetic tests performed are suitable for identification of harmful effects of particular known substances in various concentrations over different exposure of time and is considered one of the best/sensitive method to measure harmful effects of any agent of interest as compared to other physical, chemical, saprobiological, radiological methods on genetic level. The biological tests are indispensable for the evaluation of reactions of living organisms to the complex environmental pollution and for an indication of potential synergistic effects of various pollutants, while physical and chemical analyses provide nothing, but only determination of presence and concentrations of different pollutants. Moreover, they are also laborious and time consuming (Bianchi et al., 2011; Chandra et al., 2005; da Costa et al., 2012b; Guzy et al., 2012; Leme and Marin-Morales, 2008, 2009; Liman et al., 2010a; Smaka-Kincl et al., 1996; Souza et al., 2009).

A. cepa assay is efficient and reliable test systems for the rapid screening of chemicals for mutagenic and clastogenic effect evaluation (Rank and Nielsen, 1997b). This test generally provides estimate of the total toxic effect resulting from the treatment of root tip cells by mixture of wastes (Fiskesjo, 1993). The micronucleus (MN) and chromosome aberration (CA) tests provide a fast opportunity to screen genotoxic effects of chemical substances which are present in the environment. Moreover, A. cepa is being often used due to the knowledgement of its cell cycle duration and its reaction in the presence of many known mutagenic agents and on the basis of clastogenic and aneugenic effects, the action mechanism of toxic agent on genetic material can also be evaluated (Evseeva et al., 2003; Njoku et al., 2015). In situ application of $A$. cepa is easy as compared to various other biological test (Kovalchuk et al., 1998). The chromosomal features favor carrying out the CA's test, not only for toxic effect assessment, but also for understanding the action mechanisms of the test agent (Fiskejo, 1985; Rank and Nielsen, 1997b). A. cepa along with chromosome damages and cell division cycle disturbances also facilitates the evaluation of aneuploidy risks (Leme and Marin-Morales, 2008). The results of the $A$. cepa test are known to be well correlated with the data obtained from prokaryotic and eukaryotic systems (Houk, 1992; Leme and Marin-Morales, 2009). According to Fiskejo (Fiskejo, 1985), A. cepa test has similar sensitivity to that of the algal and human lymphocyte and carcinogenity assays in rodents showed the correlation of $82 \%$ with A. cepa (Rank and Nielsen, 1994). In another study, the effects of infusions of Maytenus ilicifolia Mart. and Bauhinia candicans Benth with A. cepa and bone marrow cells of Wistar rats showed similar results (Camparoto et al., 
2002). Cytotoxicities have been assessed by the $A$. cepa roots tip cell were also found similar to in vitro animal toxicity tests (Poonkuzhali et al., 2011b). In a comparative study, Ma et al. (Ma et al., 1995) reported that the A. cepa test was more sensitive and efficient for the detection of environmental pollutants. In this regard, the response of $A$. cepa and Vicia faba root was studied on the basis of chromosome length, peak sensitivity of the mitotic cells and the regions of the root tip where the MN were formed. Higher efficiency was demonstrated when the MCN frequencies were scored from the $\mathrm{F}_{1}$ cells in both $A$. cepa and Vicia treated with formaldehyde, mitomycin $\mathrm{C}$ and maleic hydrazide. The linear regression dose-response curves were established in both $A$. cepa and Vicia cell systems and the coefficients of correlations, slope values were used to verify the reliability and efficiency of these two plant cell systems. Based on the dose-response slope value, the $A$. cepa root test was proved to be more efficient test system.

The present review highlights the application of $A$. cepa for the evaluation of industrial wastewater toxicity and its efficiency in evaluating genotoxic and mutagenic agents present in industrial wastewater such as oil refinery, textile and dying industry, pulp and paper mill and metallurgy etc as well as the contaminated soil and watersheds with industrial wastewater.

\section{Endpoints studied in $A$. cepa to evaluate the toxicity}

For $A$. cepa root tip assay, the procedure and endpoints studied for toxicity monitoring are shown in Fig. 1. Endpoints are characterized by the genetic categories studied to assess the toxicity of system. For this purpose, following four microscopic genetic endpoints are reported for the assessment of toxicity in A. cepa, namely, mitotic index (MI), chromosonal aberrations (CA), nuclear aberrations (NA) and micronuclei (MN) (Leme and Marin-Morales, 2009). Additionally, some researchers have also used macroscopic characteristics such as number of root, length of root and root physical appearance for the indication of toxicity (Bianchi et al., 2011; Fazili and Ahmad, 2014; Lerda et al., 2010; Olorunfemi et al., 2011b; Olorunfemi et al., 2015b; Radić et al., 2010; Roa et al., 2012; Siddiqui et al., 2011; TORRES et al., 2014). These end points are used for the evaluation of cytotoxicity, genotoxicity and mutagenicity of the pollutant.

\section{Cytotoxicity evaluation}

The MI is very important endpoint for the evaluation of toxicity and is based on the number of dividing cell in cell cycle and mostly researchers used it as a cytotoxicity indicator (Abdel Migid et al., 2007; Carita and Marin-Morales, 2008; Leme and Marin-Morales, 2008; Porras Torres et al., 2013; Smaka-Kincl et al., 1996). For normal cell division, the MI must be equal to control and MI lowers than the control indicates the abnormality in cell division. The higher value of MI is also an indication of abnormal growth such as cell proliferation and un-control growth versus negative control (Carita and Marin-Morales, 2008; Hoshina and Marin-
Morales, 2009). So, the reduction as well as acceleration in MI are important indicators for the assessment of cytoxicity of contaminants (Leme and Marin-Morales, 2009).

The inhibition of MI may be attributed to the effect of environmental chemicals on DNA/protein synthesis of the biological system (Andrade et al., 2008; Chandra et al., 2005). A decrease in MI below 22\% versus control causes lethal effects on test organisms, while a decrease below 50\% (cytotoxic limit value) usually has sublethal effects (Abdel Migid et al., 2007). Therefore, the inhibition of root growth in fact is a measure of the inhibition of cell division, measured as a decrease in MI (Marcano et al., 2004). The decrease in MI due to the exposure of wastewater/any other physical or chemical agent indicates the presence of cytotoxic agent and can be used for the estimation of pollution level in the sample (Smaka-Kincl et al., 1996). For example, in a study to evaluate the toxicity of Pterocaulon polystachyum, Knoll et al. (Knoll et al., 2006a) correlated the inhibition of cell division to the flavonoids in the infusions tested and the authors demonstrated that with an increase in the concentration of the infusions of $P$. polystachyum, lower mitotic index values were recorded (Knoll et al., 2006a). Therefore, due to greater sensitivity of $A$. сера root to the toxic effects of physical or chemical agents in wastewater, various researchers used the MI as a cytoxicity indicator (Abu and Ezeugwu, 2008; Andrade et al., 2008; Arya and Mukherjee, 2014; Bianchi et al., 2011; Carita and Marin-Morales, 2008; Çelik and Aslantürk, 2009; Chandra et al., 2005; da Costa Machado Matos Carvalho et al., 2011; da Costa et al., 2012b; EspinozaQuiñones et al., 2009; Geras'kin et al., 2011; Hoshina and Marin-Morales, 2009; Kwasniewska et al., 2012; Olorunfemi et al., 2011b; Rodrigues et al., 2010; Souza et al., 2009). Table 1 shows the MI percentage value versus negative control in $A$. cepa cells exposed to different pollutants.

CA is characterized by change in chromosomal number or structure (Fernandes et al., 2007b). To evaluate the structural abnormalities due to toxic agent, the cell stages like prophase, anaphase, metaphase and telophase were studied well (Fiskejo, 1985; Rank, 2003; Rank and Nielsen, 1993). The CA are caused due to the DNA breakage, inhibition in DNA synthesis and altered DNA replication as a result of contact with physical and chemical polluting agents (Albertini et al., 2000). The CA include chromosome adherence, loss, breakage, bridge, irregular distribution, distortion, lagged, irregular separation, stickiness, vagrant, rings, late separation and un-orientation. Additionally, the Cmetaphase, polyploid anaphase, multipolar anaphase, polar slip, drifting away from the metaphase plate, disturbed telophase, bridge at telophase, chromatin degeneration, anaphase with multiple bridges, late anaphase stage with double bridge, disturbed telophase, bridge sticky metaphase, sticky prophase and cytokinetic failure are also the types of CA's abnormalities (Abdel Migid et al., 2007; $\mathrm{Abu}$ and Ezeugwu, 2008; Abu and Mba, 2011; Bianchi et al., 2011; ElShahaby et al., 2003; Gupta and Ahmad, 2012a; Sik et al., 2009). 


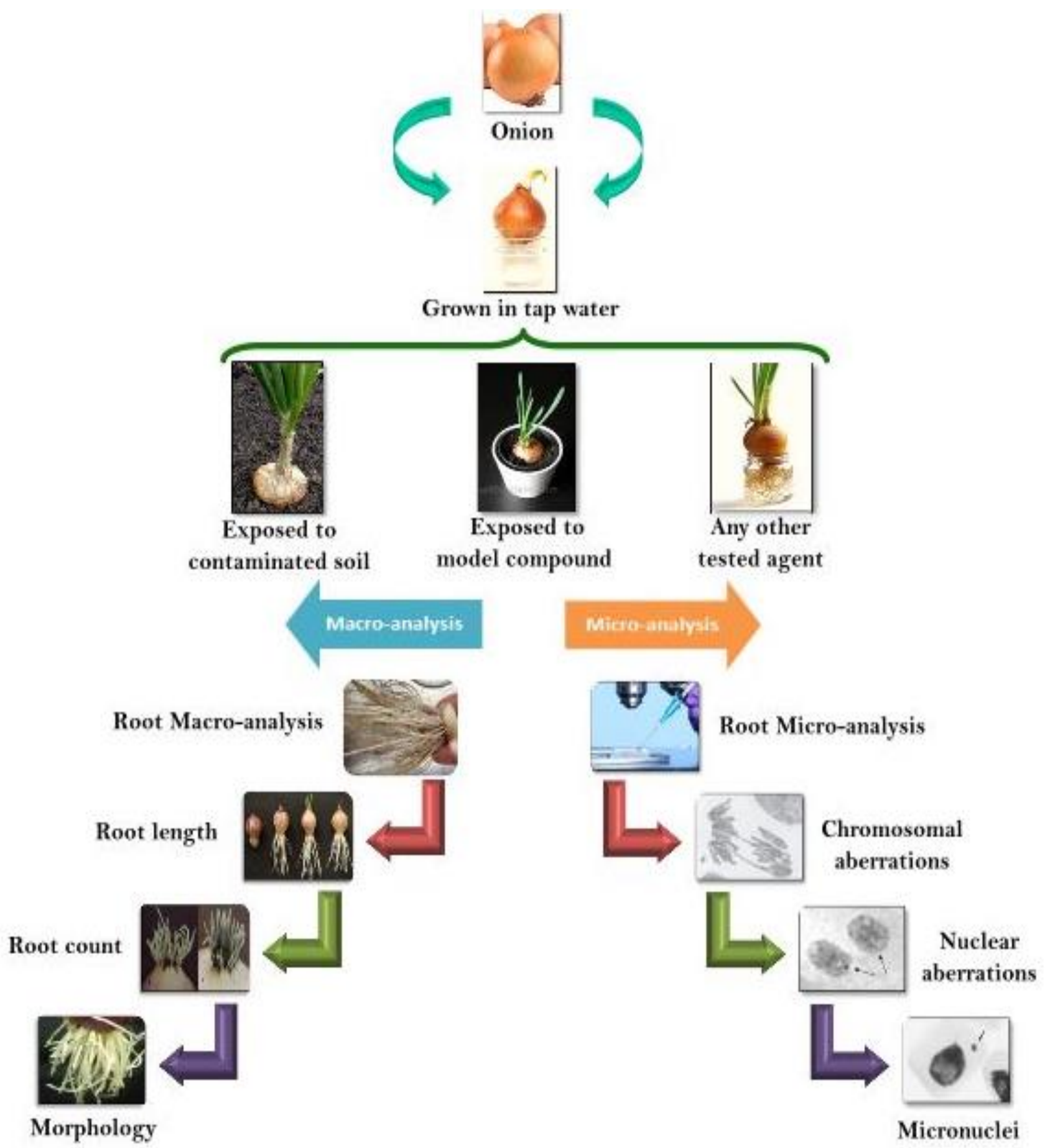

Fig. 1: Procedure and endpoints analysis in A. cepa root tip assay for toxicity monitoring

It is reported that metaphase with sticky chromosomes loses its normal appearance and is seen with a sticky "surface" causing chromosome agglomeration. Presence of such type of aberration reflects the toxic effect on chromatin, which generally leads to irreversible cell death.

Chromosomes stickiness is attributed to the formation of complexes of toxic agent with phosphate groups in DNA, on DNA condensation or on formation of inter-and- intra-chromatid crosslinks (El-Ghamery et al., 2003). Additionally, the late segregation of chromosome, Cmetaphases and multipolar anaphases suggest the effect on microtubule assembly. The microtubules perform central role during the growth and mitotic cycle such as chromosome migration, cell structure and formation of cell wall (Jordan and Wilson, 1998). 

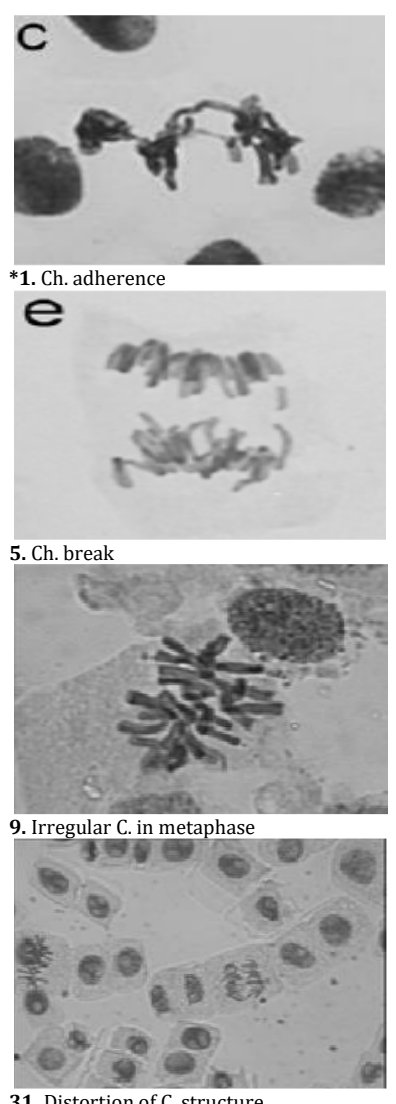

31. Distortion of C. structure

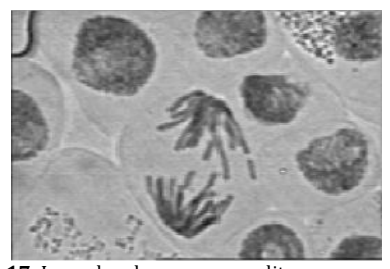

17. Irregular chromosome splits

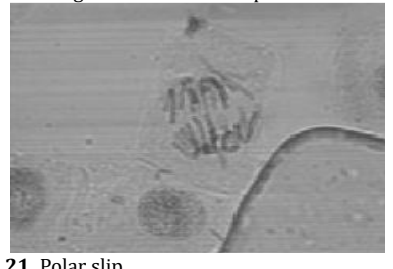

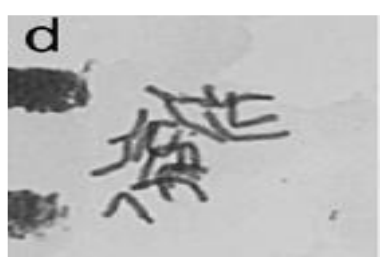
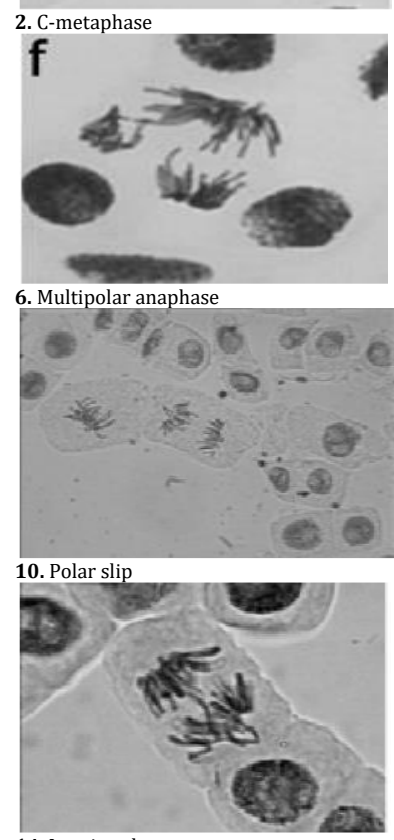

14. Lagging chromosome

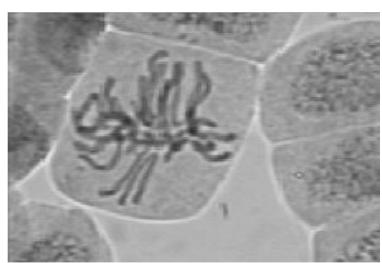

18. Irregular metaphase

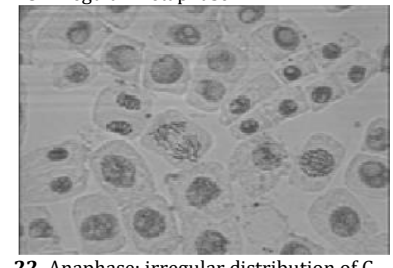

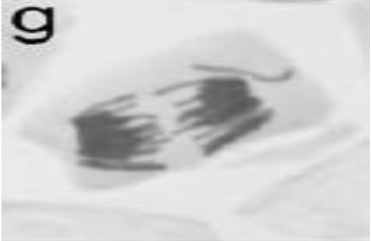
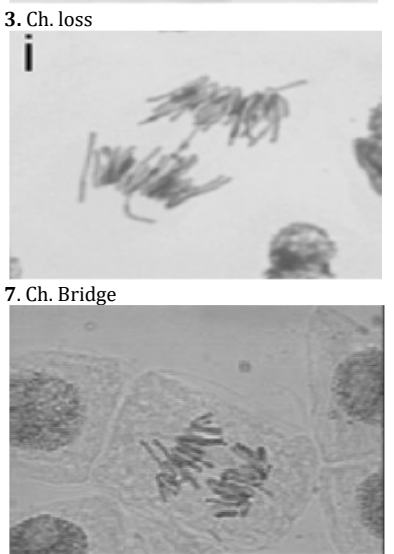

11. Irregularities anaphase

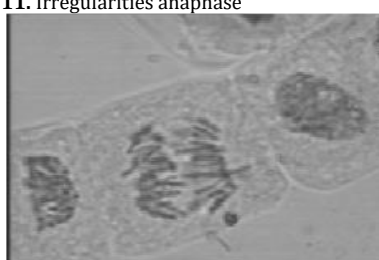

15. Irregular distribution of $C$

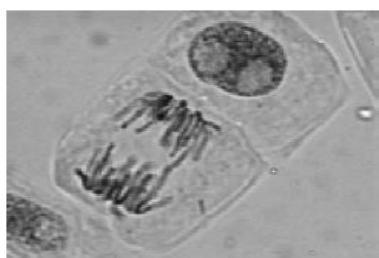

19. Anaphase: C. bridge

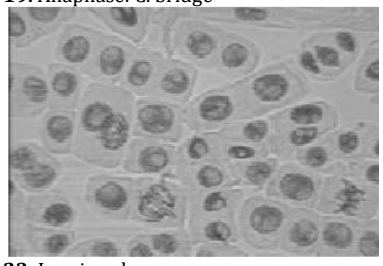

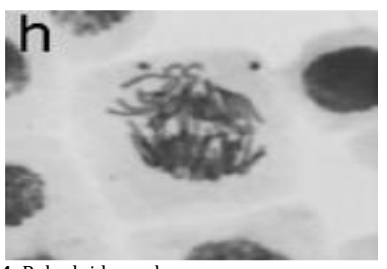

4. Polyploid anaphase

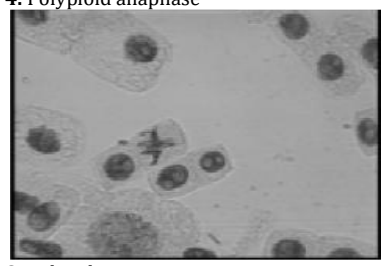

8. Polar slip

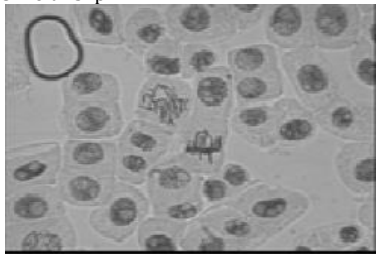

12. Polar slip, irregular distribution of $\mathrm{C}$

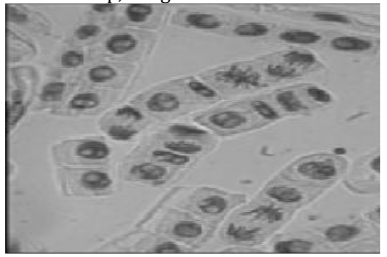

16. Drifting away from the metaphase plate

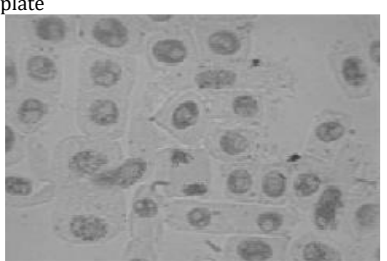

20. Chromosome stickiness

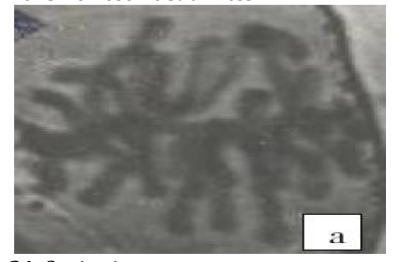

Fig. 2: Chromosomal aberrations and their types observed in A. cepa meristematic cells exposed to different genotoxic agents (1-164). * References and physiochemical properties of wastes: 1-7 (Bianchi et al., 2011): River water mixed with industrial effluents: pH-6.83-7.29, EC-29-114, DO-4.43-7.09 mg/L, T-24.1-25.9 ${ }^{\circ} \mathrm{C}$, metal (Cu, Cr, Ni, Cd, Zn, Fe, Mn, Ca). 8-15 (Sik et al., 2009): the water incoming (10\%) and outgoing at biological and chemical wastewater treatment from industrial zone (COD-14.18, SS-270.0, Cr-0.62, Pb-1.75, Cu-2.31, Zn-1.81 mg/L, pH-8.6). 16-23 (Sik et al., 2009): the water incoming (25\%) and outgoing at biological and chemical wastewater treatment in organized industrial zone (COD-14.18, SS-270.0, Cr-0.62, $\mathrm{Pb}-1.75, \mathrm{Cu}-2.31, \mathrm{Zn}-1.81 \mathrm{mg} / \mathrm{L}$, pH-8.6). 24-25 (Abu and Ezeugwu, 2008): chemical industry pH-6.3, TDS-520, SO-24-71.7, Cl--90, COD-512, TSS-14700, Na-275, K-42.5, Ca-1062, Mg-48.6, Co-18.85, Cu-1191.11, Cd-43.75, Pd-2.30 mg/L. 26-30 (El-Shahaby et al., 2003): industrial wastewater. 31-33 (Abu and Mba, 2011): pharmaceutical (cotrimoxazole, chlorphiramine, paracetamol, ascorbic acid, ampiclox ampicillin and cloxiacillin). 34-39 (Abdel Migid et al., 2007): industrial effluent before algal treatment. 40-43

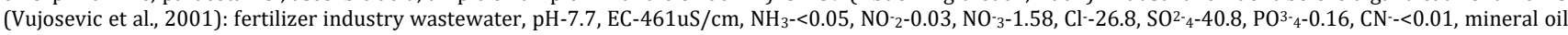
$(0.045) \mathrm{mg} / \mathrm{L}$ and Fe-0.03, Mn, Pb, Cd, Zn, Cr, Hg, As, Cu, $<0.05 \mathrm{mg} / \mathrm{L}$ and dichlorethane- $<0.1)$, toluene-2.8, xylene-2.8, PCB- $<0.001 \mathrm{and} \mathrm{PAH's-0.02} \mathrm{ug/L.} \mathrm{44-50}$ (Odeigah et al., 1997): petroleum wastewater (44-50). 51-58 (Gupta and Ahmad, 2011). 59-63 (Kovalchuk et al., 1998): soil contaminated with radiaoactive material in Chernobyl accident. 64-66 (Kwasniewska et al, 2012): landfill leachates: $\mathrm{Pb}, \mathrm{Ni}, \mathrm{Cd}, \mathrm{Hg}$, benzene, dichloromethane, chloroform, trichlorobenzenes, exachlorobenzene, hexachlorobutadiene, hexachlorocyclohexane, lindane, hexachlorocyclohexane, pentachlorobenzene, pentachlorophenol, PAH's. $67-76$ (Leme et al., 2008): refinery wastewater: $\mathrm{HC}\left(\mathrm{C}_{12}-\mathrm{C}_{18}\right)$ and PAH's (naphthalene, acenaphthylene, acenaphthlene, fluorine, phenanthrene, pyrene, benzoanthracence and chrysene). 77-86 (Mazzeo et al., 2011): mixture of benzene, toluene, ethylbenzene and xylene. 87-98 (Ventura-Camargo et al., 2011): azo dye. 99-104 (Jadhav et al., 2010): textile effluent. 105-108 (Kumari et al., 2009) 2009: silver nano particle. 109-112 (Herrero et al., 2012): Di(2-ethylhexyl)phthalate, triclosan and propylparaben. 113-119 (Ukaegbu and Odeigah, 2009). 120-123 (Gul et al., 2006): 0.1\%, 0.2\% and 0.4\% concentrations of herbicide avenoxan. 124-129 (Olorunfemi et al., 2011): A. cepa grown in agriculture filed under the effect of pesticides. 130-135 (Ivanova et al., 2005): heavy metal and cyanide contaminated river waters in a mining region. 136-138 (Quilang et al., 2008): polychlorinated biphenyls. 139-144 (Radic et al., 2010). 145-147 (Oriaku et al., 2011): textile effluent (BOD-152, COD319, TSS-1160, 0 \& G-612, pH-6.9, Cr-1.20, Cu-0.02, NH+4-0.50 mg/L). 148-151 (Pavlica et al., 2000): wastewater from the phosphoric gypsum depot: Ca2+(960), S042-(3599), $\mathrm{P}^{+}+(3200-3800), \mathrm{F}-(2500-3500), \mathrm{Si}^{4+}(800), \mathrm{Na}^{+}(1313), \mathrm{Fe}^{3+}(55), \mathrm{Mg}^{2+}(114), \mathrm{NH}^{4+}(23), \mathrm{Cl}-(40), \mathrm{K}^{+}(53)$ and SS-9 mg/dm³. $152-156$ (Ivanova et al., 2008$)$. 157-160 (Saxena et al., 2005): 161-164 (Staykova et al., 2005). 

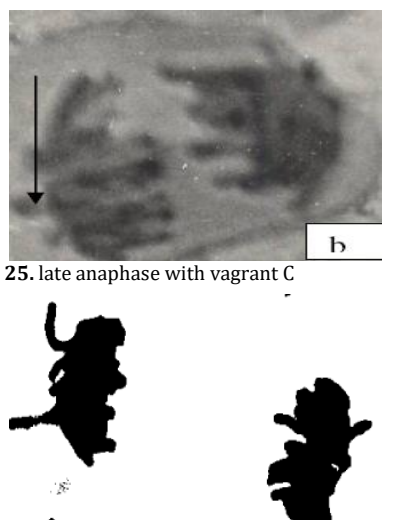

29. Disturbed telophase
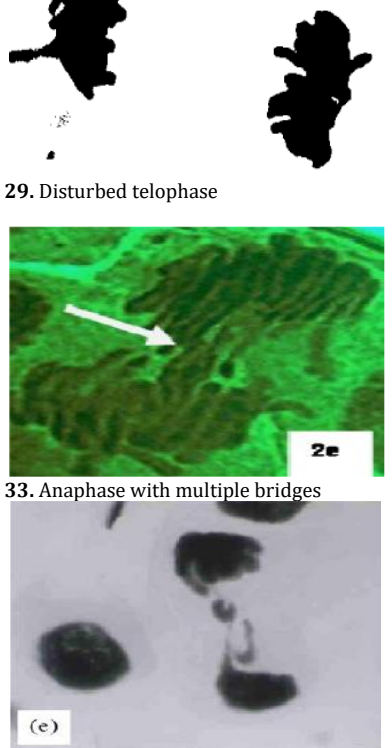

37. Disturbed anaphase: unoriented C

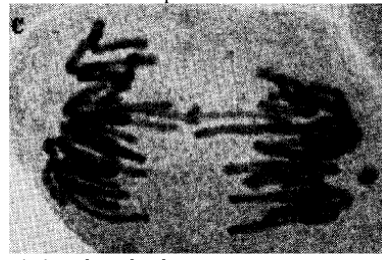

41. Anaphase bridge

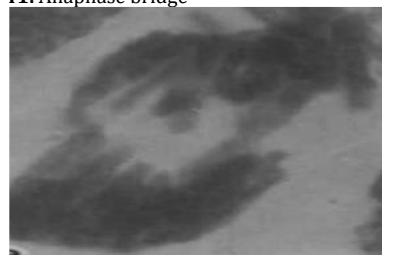

45. Sticky anaphase: bridge \& fragments

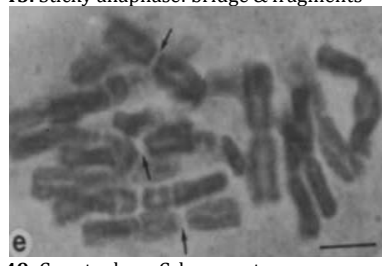

49. C-metaphase C. long centromeres

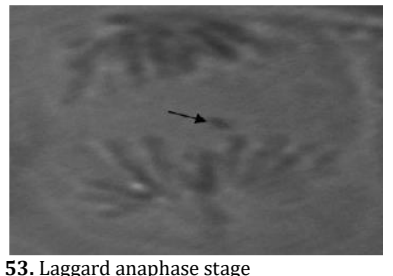

53. Laggard anaphase stage

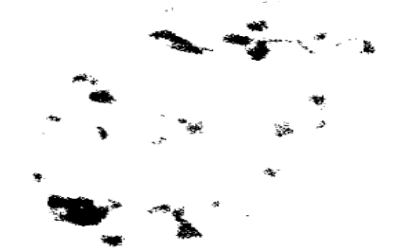

26. Ch. ring

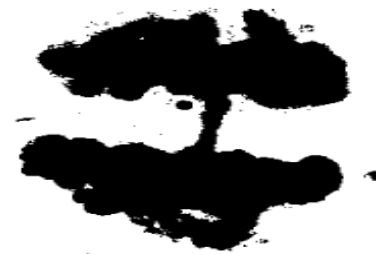

30. Bridge at telophase

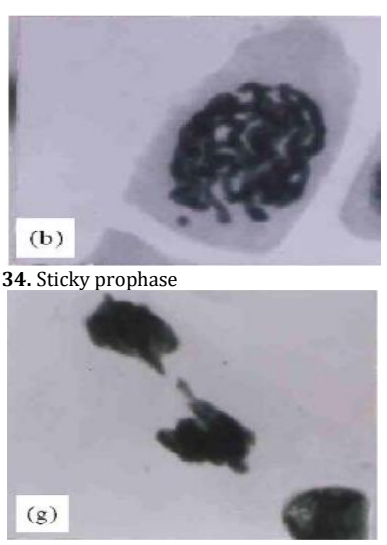

38. Bridge at telophase

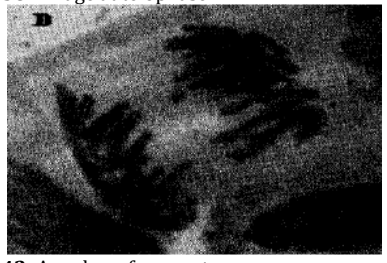

42. Anaphase fragment

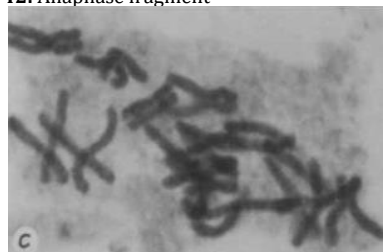

46. C-metaphase

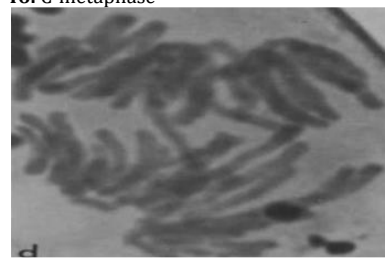

50. Multipolar anaphase: bridges \& fragments

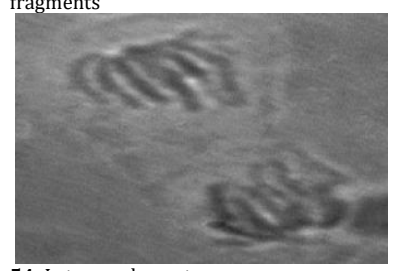

54. Late anaphase stage

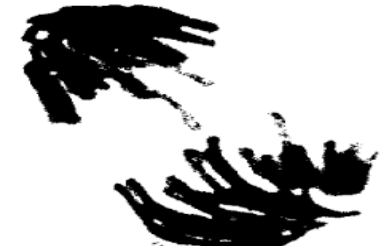

27. Anaphase: late separation of $C$

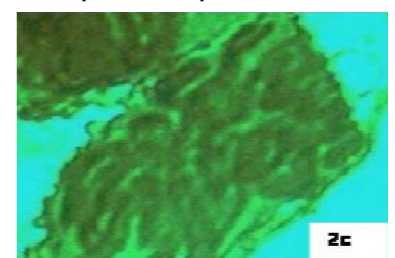

31. Abnormal cells, Diverse forms of Cmitotic

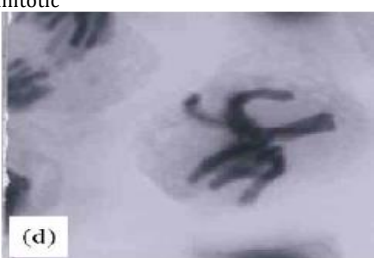

35. Sticky metaphase

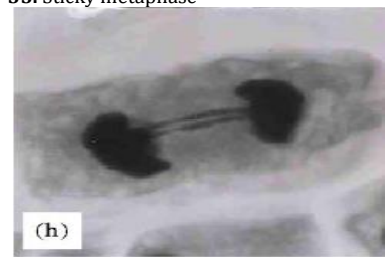

39. Late separation at telophase
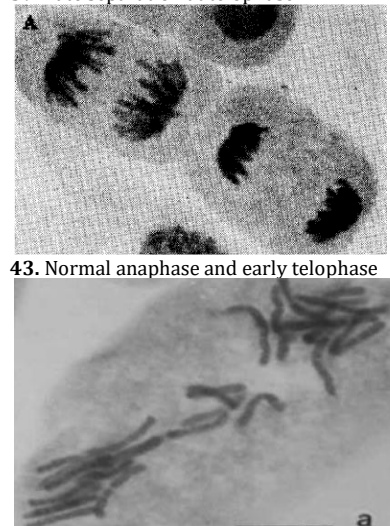

47. C-anaphase with laggards C

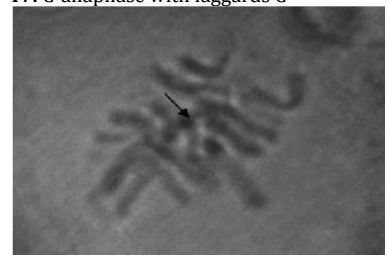

51. Metaphase: sticky chromosomes

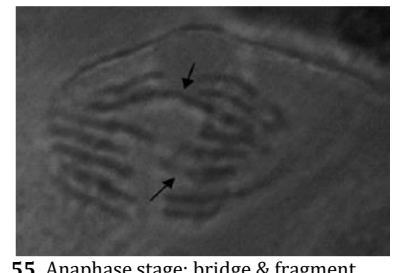

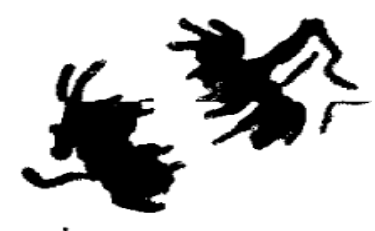

28. Disturbed anaphase

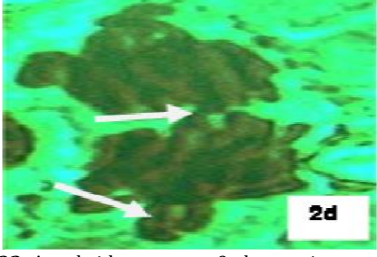

32. Ana: bridge, vagrant \& chromatin degeneration

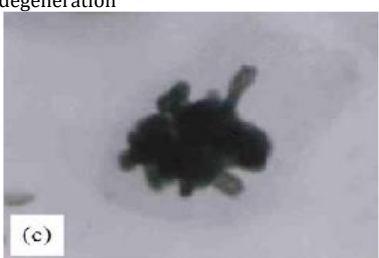

36. Disturbed metaphase: unoriented Ch

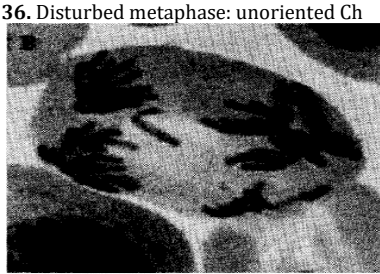

40. Multipolar anaphase

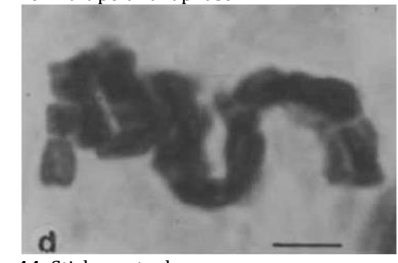

44. Sticky metaphase

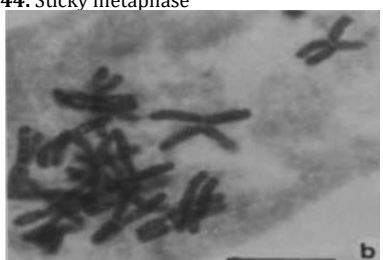

48. Vagrant chromosome

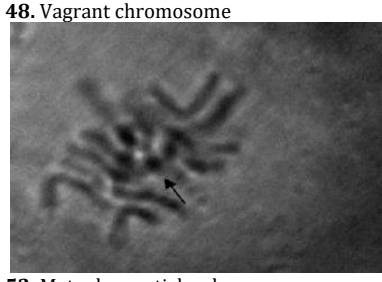

52. Metaphase: sticky chromosomes

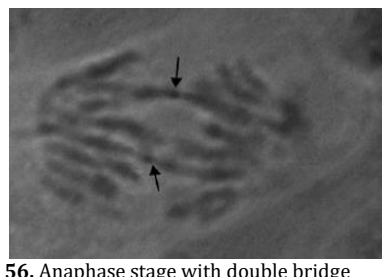

Fig. 2: Continue... 


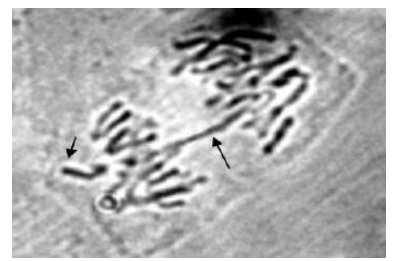

57. Anaphase stage: bridge \& C. loss

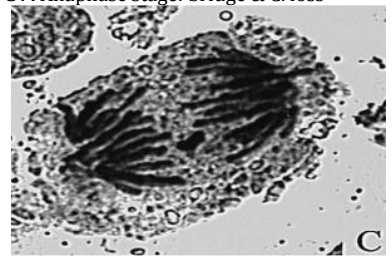

61. Anaphase cell with a fragment $\triangle \mathrm{C}$

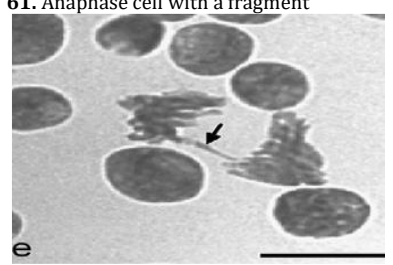

65. Single anaphase bridge

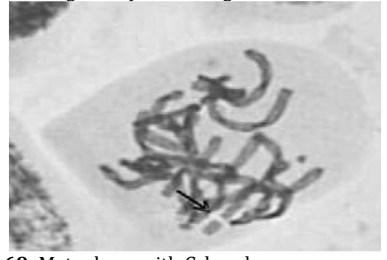

69. Metaphase with C. break

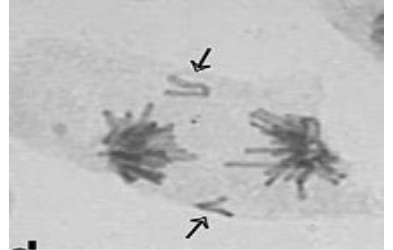

73. Anaphase with C. losses

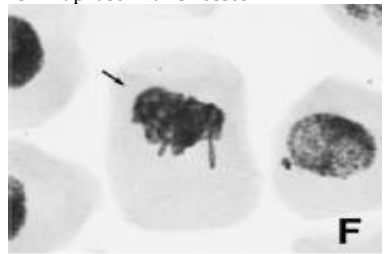

77. Metaphase with adherence

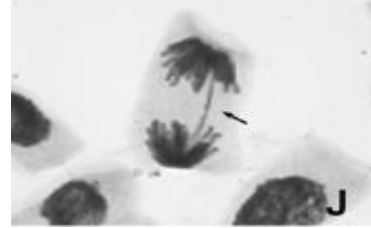

81. Anaphase with chromosomal bridge

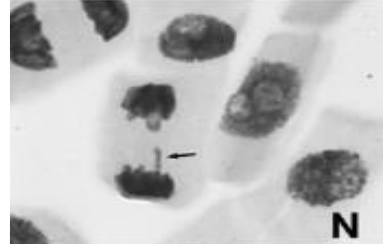

85. telophase with C. laggard
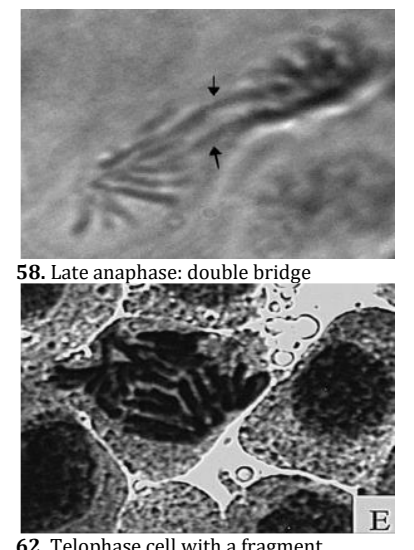

62. Telophase cell with a fragment

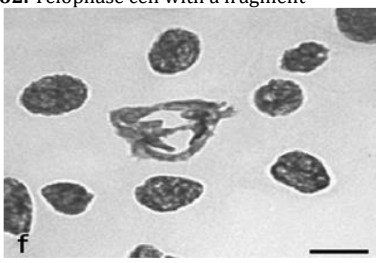

66. Three anaphase bridges

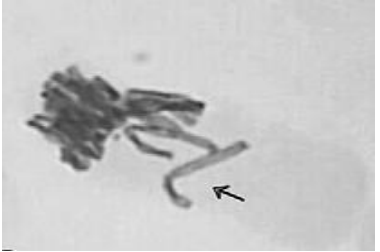

70. Metaphase with C. loss

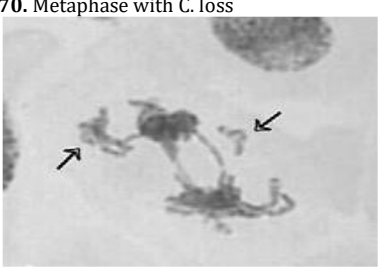

74. Multipolar anaphase bridge \& C. loss

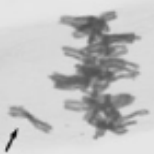

G

78. Metaphase with C. loss

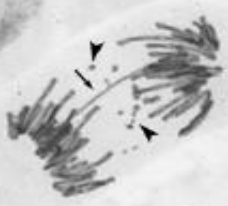

82. Anaphase with bridge \& break

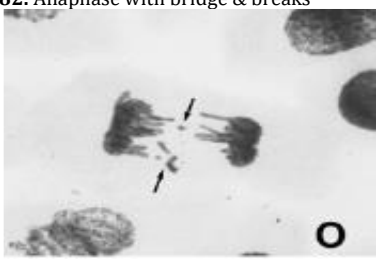

86. Telophase with C. break
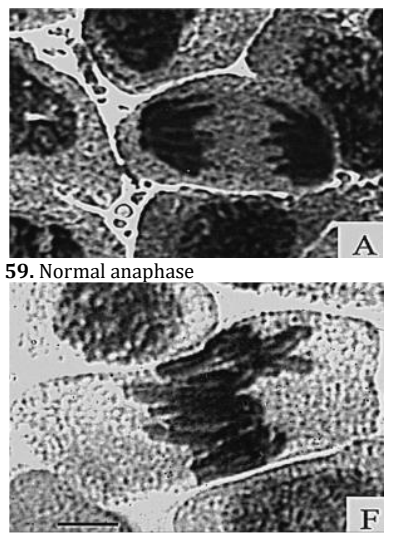

63. Vagrant chromosomes

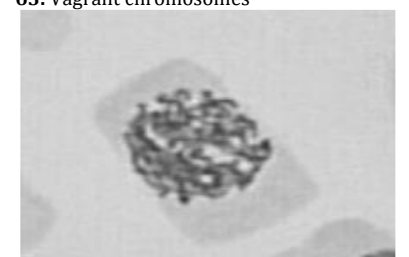

67. Normal prophase
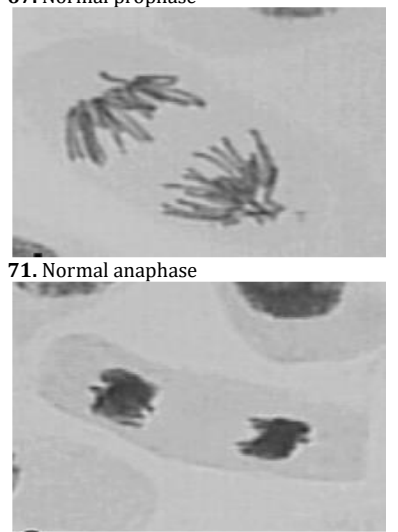

75. Normal telophase

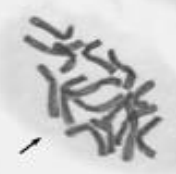

79. C-metaphase

3

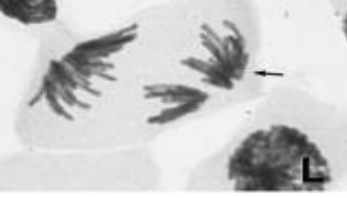

83. Multipolar anaphase

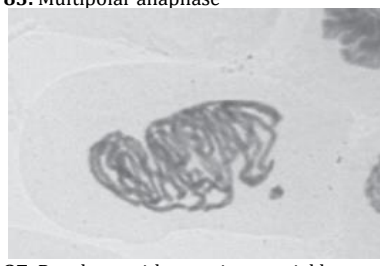

87. Prophase with genetic material loss

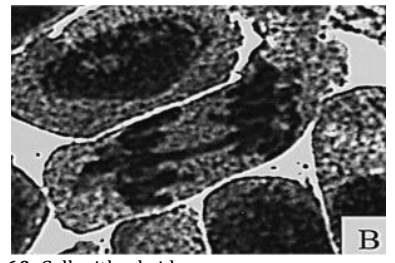

60. Cell with a bridge

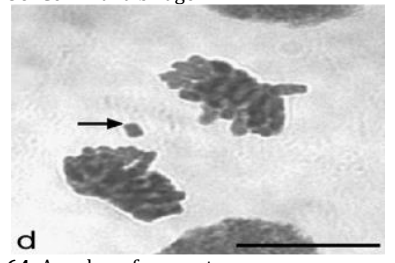

64. Anaphase fragment

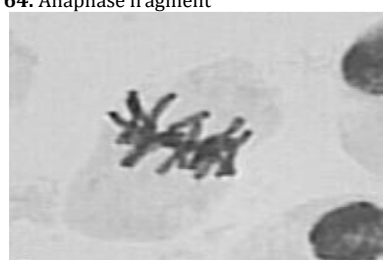

68. Normal metaphase

$2 \pm 35$

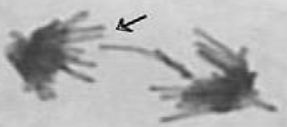

72. Anaphase with C. bridge
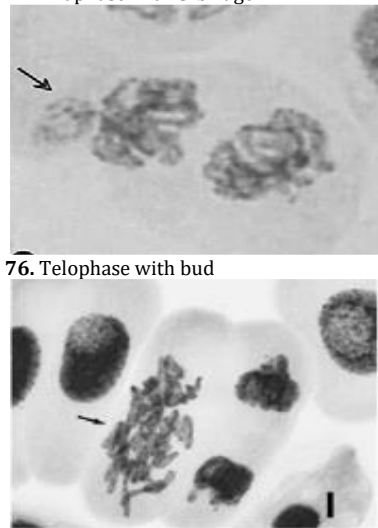

80. Polyploid metaphase with adherence

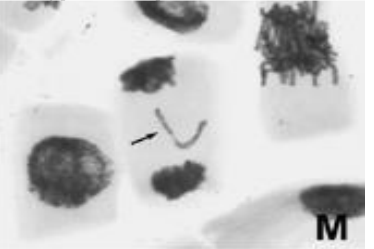

84. Telophase with C. loss

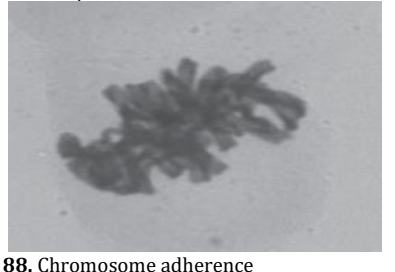

88. Chromosome adherence

Fig. 2: Continue... 
In the C-metaphase number of chromosomes, late segregation and multipolar anaphases are attributed to alterations and disturbances in the dynamics of the microtubules that is also called a spindle poisoning (Andrade et al., 2008; Haroun and Al Shehri, 2001). The C-metaphases are evidence of aneugenic agents, since they provide the complete inactivation of the cell mitotic spindle (Fiskejo, 1985). The spindles are inactivated when no equatorial plate is organized and consequently, the centromere division is blocked (Leme et al., 2008). According to (Fernandes et al., 2007b) and (Kirsch-Volders et al., 2002), the presence of Cmetaphases can result in multinuclear cells, although the most frequent result is the induction of MN.

Some authors reported that chromosomal losses, breaks as well as the excess materials, promoted by the DNA replication, can also induce $\mathrm{MN}$, which can be eliminated from the cell in the form of mini cells, i.e., small cytoplasm portions with a reduced fraction of nuclear material (Fernandes et al., 2007b; Leme et al., 2008) and resultantly CA's types are induced from nuclear abnormalities such as nuclear buds, MN, mini cells, lobated nuclei and polinucleated cells (Fernandes et al., 2007b).

It is reported that the CA's aberration such as breaks and fragments are induced due to formation of DNA-DNA and DNA-protein cross-links and heavy metals are considered as major contributor in this regard (Chandra et al., 2005). The mechanisms of CA involve clastogenic and aneugenic actions. Clastogenic action is characterized by the induction of chromosomal breakage during cell division, while aneugenic action comprises the inactivation of a cell structure such as the mitotic spindle, leading to chromosomal losses (Fenech, 2002; Leme and Marin-Morales, 2009).

The chromosomal adherence is another common sign of toxic effects on the genetic material and may cause irreversible effects on the cell, triggering the cell death process (Fiskesjo, 1993; Turkoglu, 2007) and can lead to develop chromosomal bridges and thereby, chromosomal breaks (Marcano et al., 2004). The chromosomal bridges resulted from adherence which can multiply and persist until telophase stage (Leme and Marin-Morales, 2008). Furthermore, chromosomal adherence is also associated with the formation of chromosomal bridges and eventually lead to chromosomal breaks (Marcano et al., 2004). The multipolar anaphases result from a disfunction of the mitotic spindle, which lead to unbalanced chromosome distribution, heading them for more than two poles, opposite to what occurs in the normal division cycle (Rank and Nielsen, 1998).

To evaluate the CA by using $A$. cepa is not a easy task, since it requires an accurate, adequate and precise knowledge of cell division phases and their possible abnormalities (Leme and Marin-Morales, 2009). Before the work of (Fiskejo, 1985; Rank and Nielsen, 1993), the CA applications for the estimation of polluting agent were uncommon. Afterword, a number of authors used CA's in A. cepa to detect the toxicity of wastewater samples of various origin (Andrade et al., 2008; Bernardes et al., 2015; Carita and Marin-Morales, 2008; da Costa Machado Matos Carvalho et al., 2011; Firbas and Amon, 2013; Geras'kin et al., 2011; Holan, 2014; Kwasniewska et al., 2012; Leme et al., 2008; Leme and MarinMorales, 2008; Mazzeo et al., 2011a; Mishra, 1993; Monarca et al., 2000; Phugare et al., 2011; Tabrez and Ahmad, 2011; Wardini and Notodarmojo, 2015).

Table 1 shows the CA's percentage value versus negative control in A. cepa cells exposed to different pollutants, while the pictorial presentation of CA's can be seen in Fig. 2.

\section{Genotoxicity evaluation}

The toxicity evaluation on the basis of NA endpoint was started in the beginning of present decade and is characterized by morphological changes in nuclei during cell division. The NA include bi-nucleated, multi-nucleated cell, lobulated nuclei, nuclei carrying nuclear buds, mini cell, vacuolated nucleus, nucleus with nuclear wall lesions and deformed nuclei. The NA evaluation is a sensitive analysis to detect the toxic effects of any mutagen (Abdel Migid et al., 2007; Abu and Ezeugwu, 2008; $\mathrm{Abu}$ and Mba, 2011; Akinsemolu et al., 2015; Bianchi et al., 2011; Chandra et al., 2005; Hoshina and Marin-Morales, 2009; Leme and MarinMorales, 2009).

The lobated nuclei can result from multipolar anaphases with chromosomal bridges and the presence of multipolarity during the nuclear division seems not to avoid the reorganization of the nuclear envelope and the membrane would follow the unbalanced distribution of the genetic material within the cell, resulting in the lobated nuclei and polynuclear cell (Leme et al., 2008). The presence of NA leads to the induction of cell death process (Leme et al., 2008; Leme and Marin-Morales, 2008). The nuclear bud is another NA type and is considered among the most frequent abnormality. The nuclear bud is well known genotoxic alteration and its formation is related to the initiation of the nuclear envelope formation prior to the total migration of the chromosomes to the opposite poles and consequently, their incorporation into the nuclei. The nuclear bud may originate as a result of chromosome breaks, bridges and rearrangements due to clastogenic action of agents, which hinder the proper reorganization of the chromatin in the nucleus. The nuclear buds may also be the result of cellular activities that promote the elimination of the amplified genetic material (Mazzeo et al., 2011a; Shimizu et al., 2000). Various researchers used this end point for genotoxicity evaluation of pollutants (Asita and Makhalemele, 2009; Chaparro and Pires, 2015; Olorunfemi et al., 2014a; Olorunfemi and Ehwre, 2011; Paiva et al., 2008; Phugare et al., 2010)

Fig. 3 shows the NA's formation reported in literature in the A. cepa root cells after exposure to toxic agents, while percentage NA's value versus negative control can be seen in Table 1 .

\section{Mutagenicity evaluation}



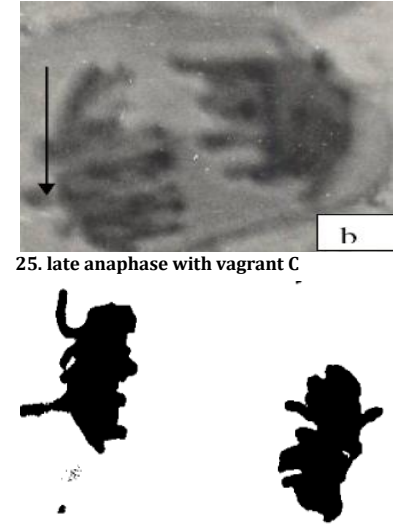

29. Disturbed telophase
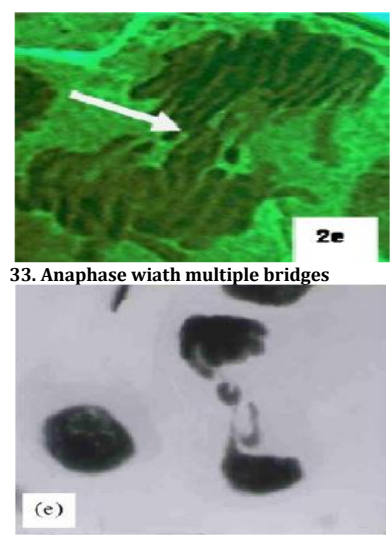

37. Disturbed anaphase: unoriented C

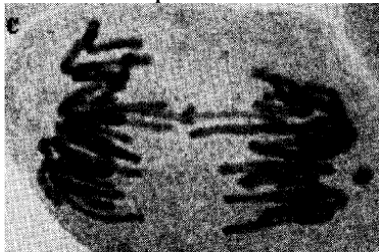

41. Anaphase bridge

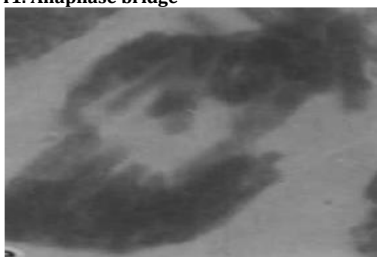

5. Sticky anaphase: bridge \& fragments

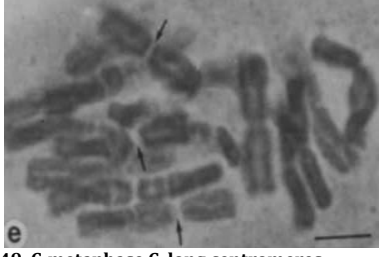

9. C-metaphase C. long centromeres

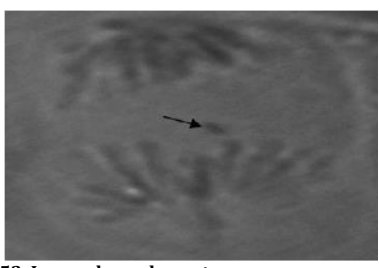

53. Laggard anaphase stage

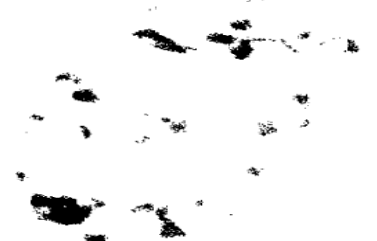

26. Ch. ring

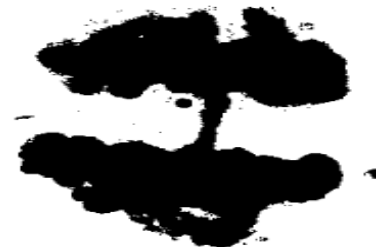

30. Bridge at telophase

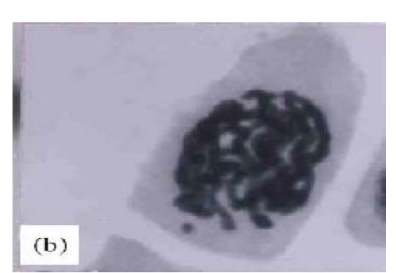

34. Sticky prophase

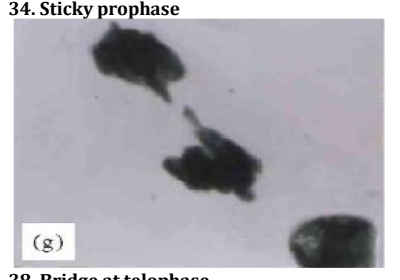

38. Bridge at telophase

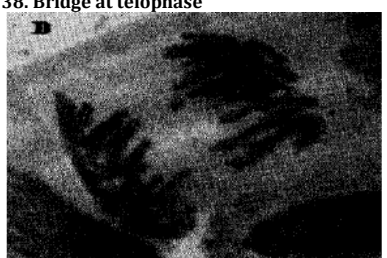

42. Anaphase fragment

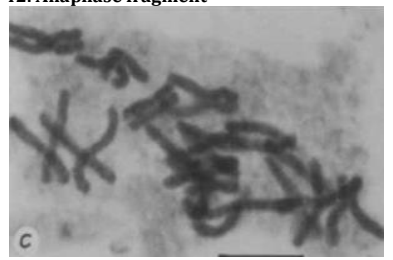

46. C-metaphase

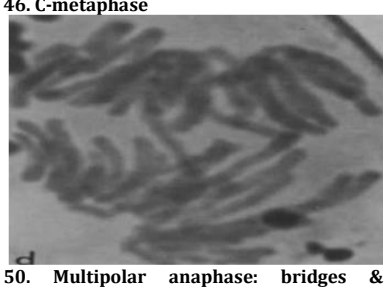
fragments

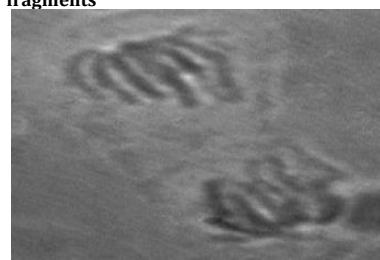

54. Late anaphase stage

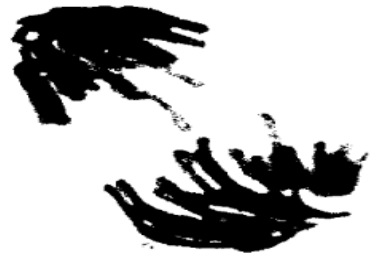

27. Anaphase: late separation of $\mathrm{C}$

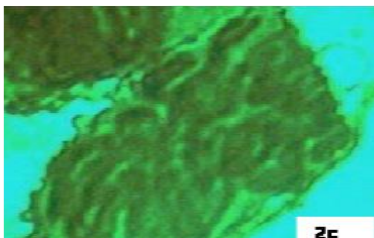

1. Abnormal cells, Diverse forms of $C$ mitotic

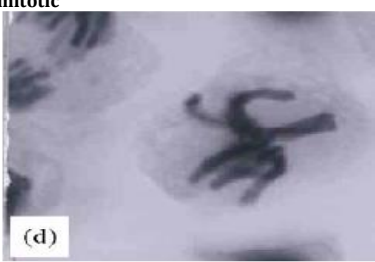

35. Sticky metaphase

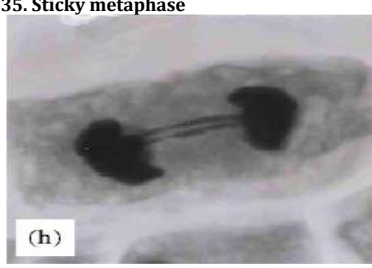

9. Late separation at telophase

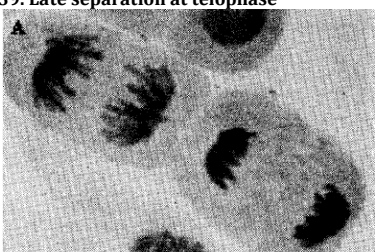

43. Normal anaphase and early telophase

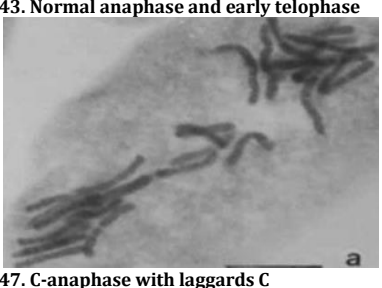

47. C-anaphase with laggards $C$

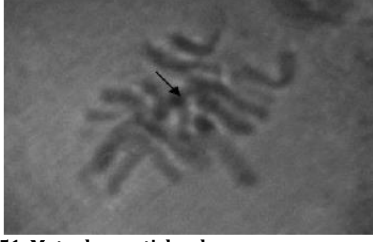

1. Metaphase: sticky chromosomes

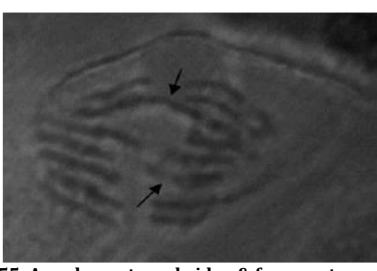

55. Anaphase stage: bridge $\&$ fragment

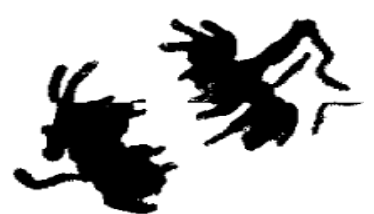

28. Disturbed anaphase

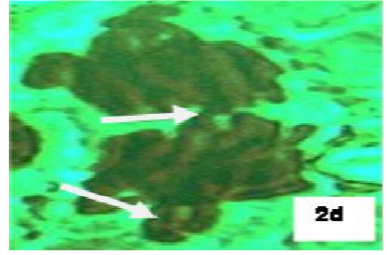

32. Ana: bridge, vagrant \& chromatin degeneration
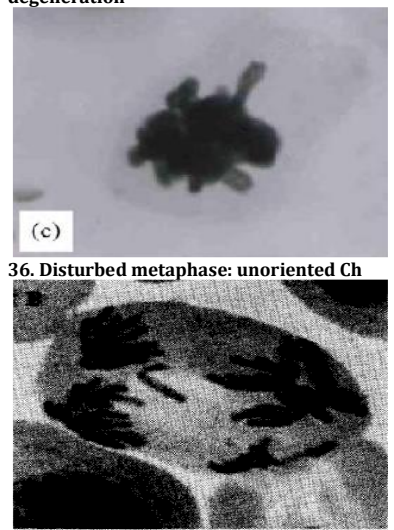

40. Multipolar anaphase
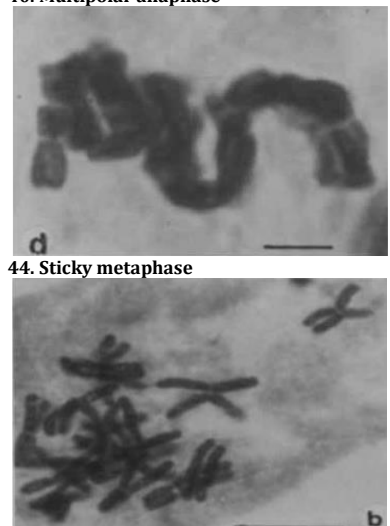

48. Vagrant chromosome

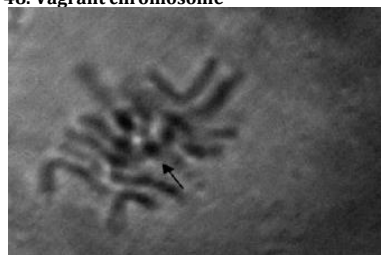

52. Metaphase: sticky chromosomes

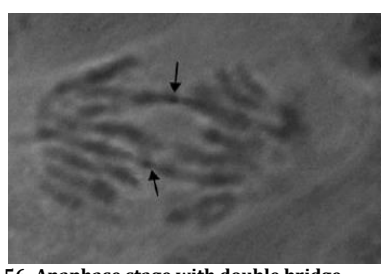

56. Anaphase stage with double bridge

Fig. 2: Continue... 
ISSN: 2410-9649
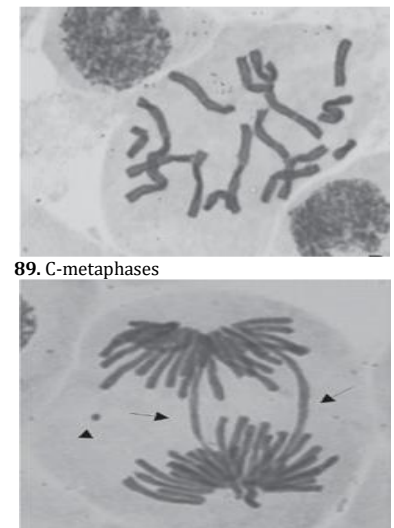

93. C. bridges and C. fragment

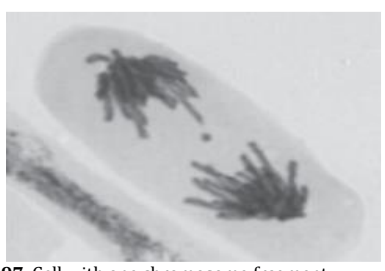

97. Cell with one chromosome fragment
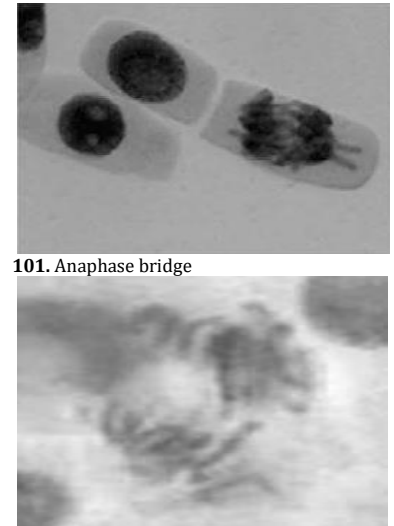

105. Chromatin bridge

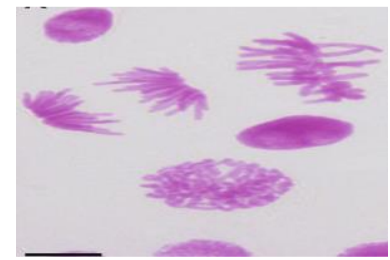

109. Normal mitotic stages

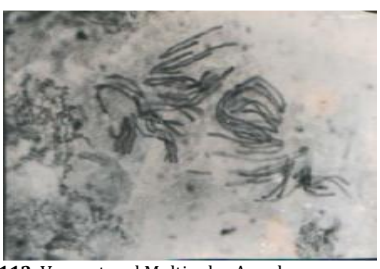

13. Vagrant and Multipolar Anaphase

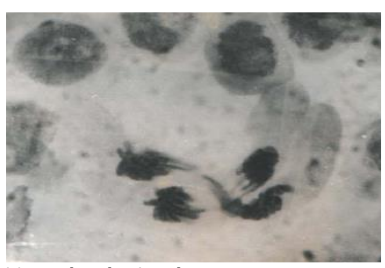

117. Multipolar Anaphase
Iqbal et al / Chemistry International 5(1) (2019) 1-80
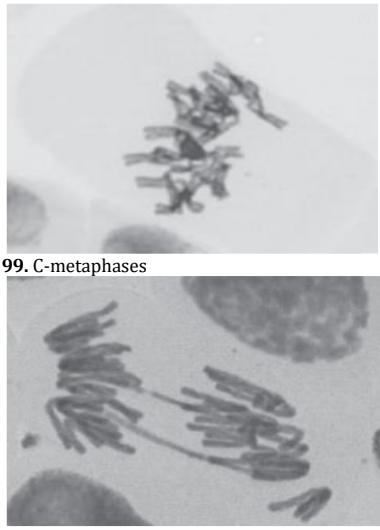

94. Chromosome losses and bridge

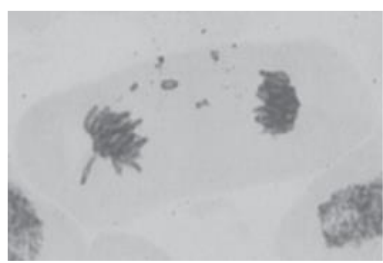

98. Cell with chromosome fragments

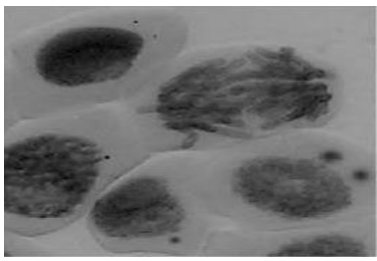

102. Anaphase bridge

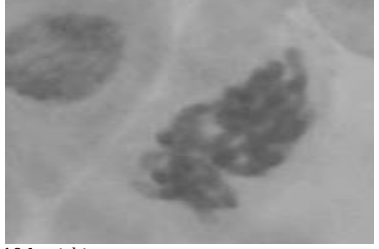

106. stickiness

C

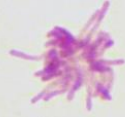

110. Sticky metaphase

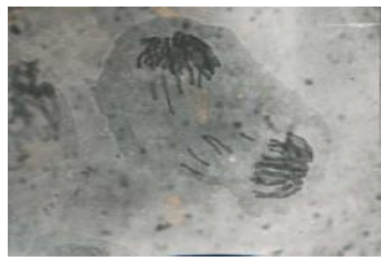

114. Telophase with laggards

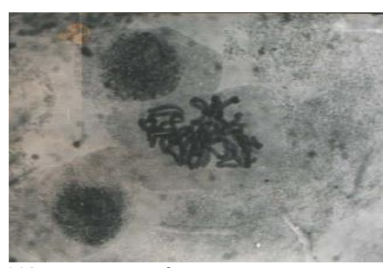

118. Vagrant Metaphase

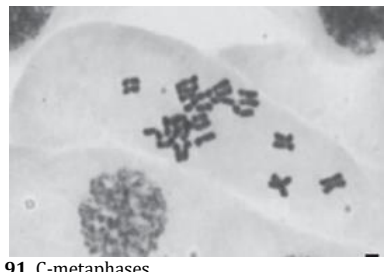

91. C-metaphases

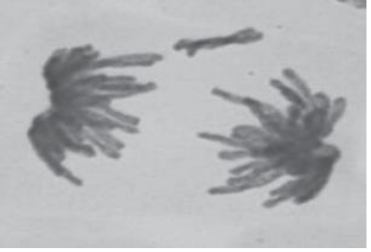

95. Chromosome losses

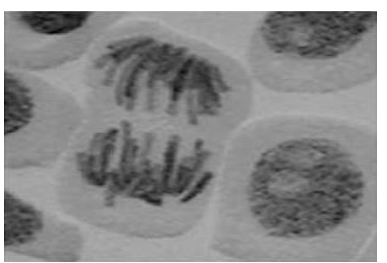

99. Normal anaphase cell

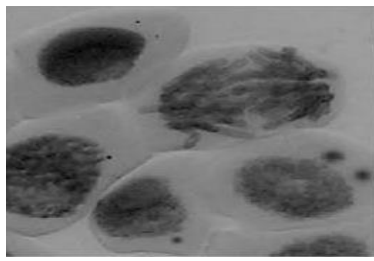

103. Vagrant chromosome in telophase

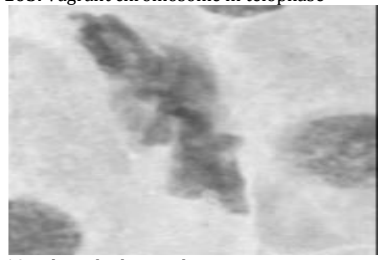

107. disturbed metaphase

D

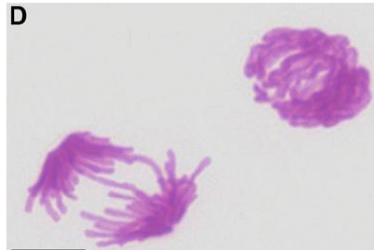

111. Chromosome bridges

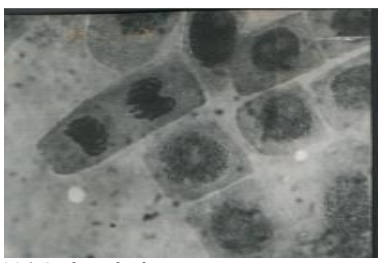

115. Sticky Telophase

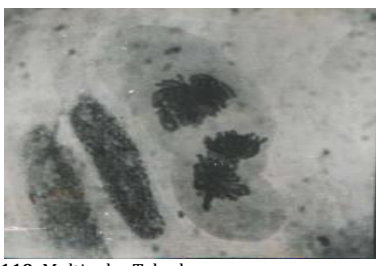

119. Multipolar Telophase iscientic.org.

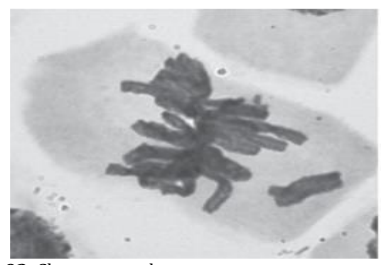

92. Chromosome losses

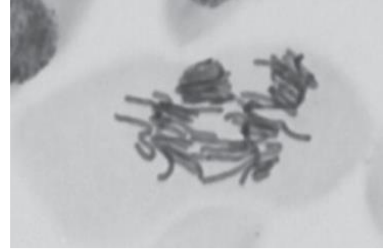

96. Multipolar anaphase

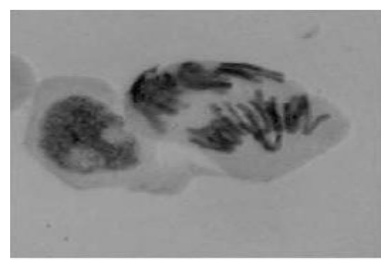

100. Fragment in anaphase
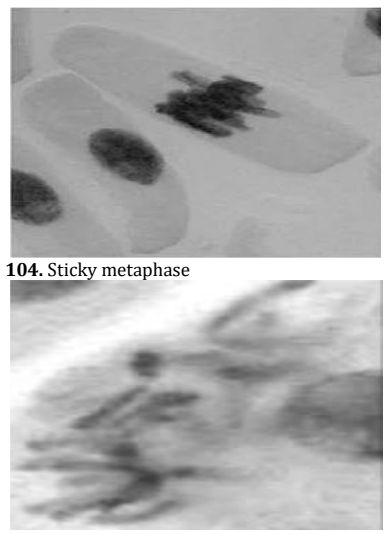

108. Multiple chromosomal break

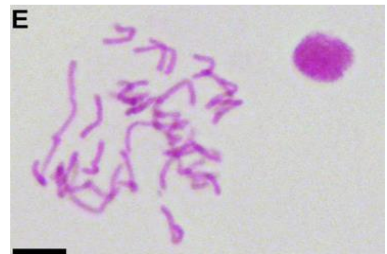

112. Chromosome mis-segregation

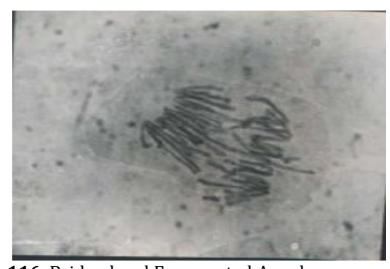

116. Bridged and Fragmented Anaphase

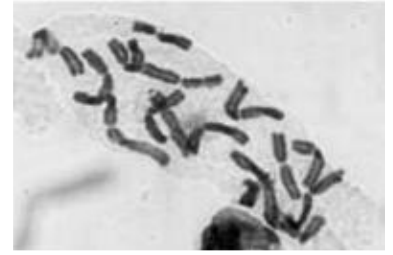

120. c-Mitosis

Fig. 2: Continue... 

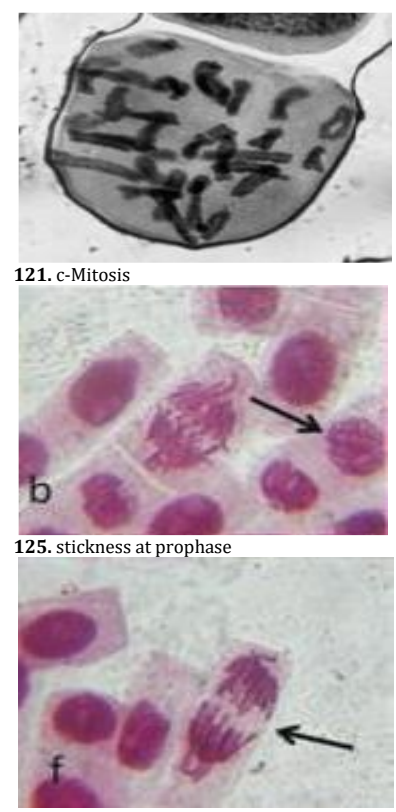

129. bagrants
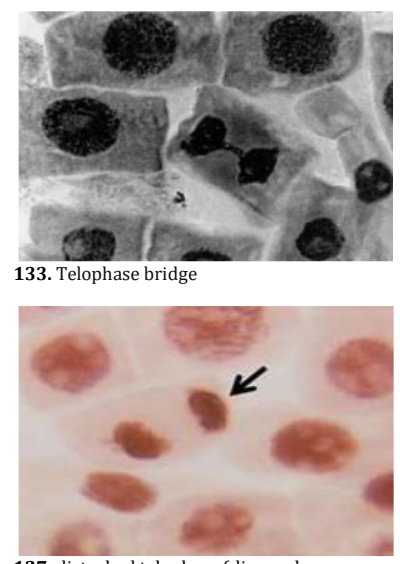

137. disturbed telophase (diagonal orie
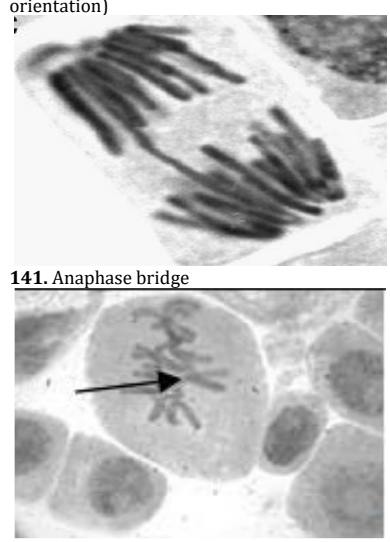

145. Abnormal metaphase (attached chromosome)

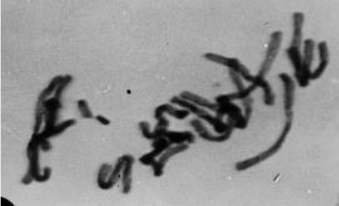

149. fragments in metaphase

Fig. 2: Continue...

Iqbal et al / Chemistry International 5(1) (2019) 1-80

iscientic.org.

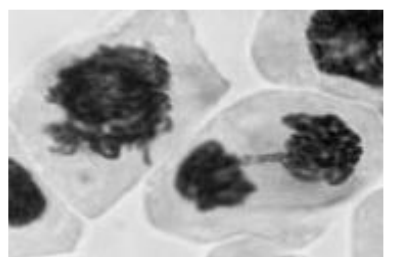

122. Bridge and stickiness in telophase
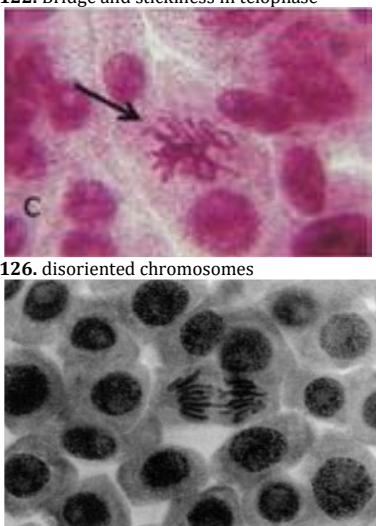

130. Anaphase bridge and fragments

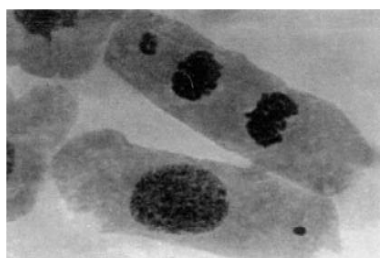

134. Telophase with a lagging chromosome

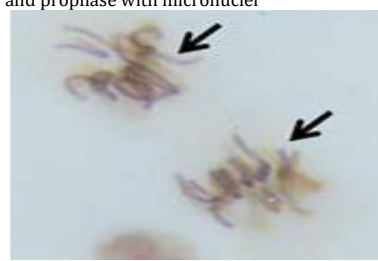

138. disturbed metaphase
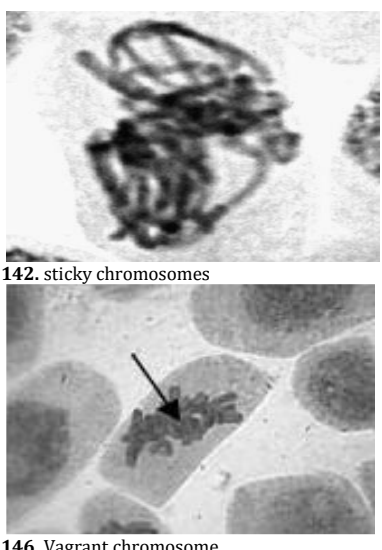

146. Vagrant chromosome

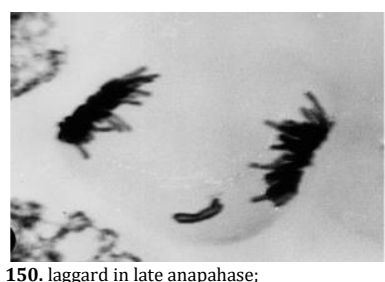

150. laggard in late anapahase;

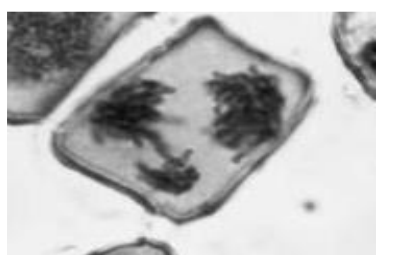

123. Multipolar telophase and bridge
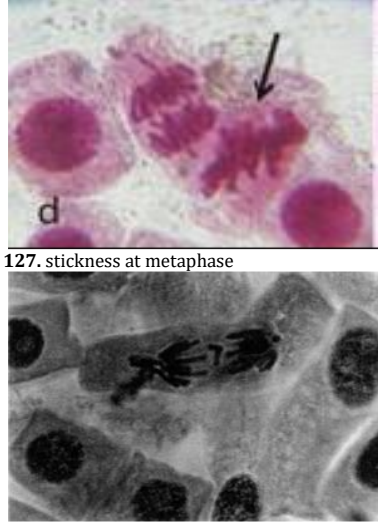

131. Anaphase with fragments

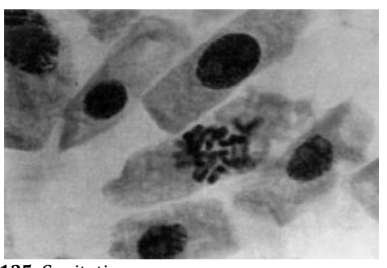

35. C-mitotic

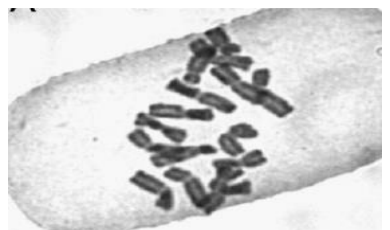

139. c-metaphase
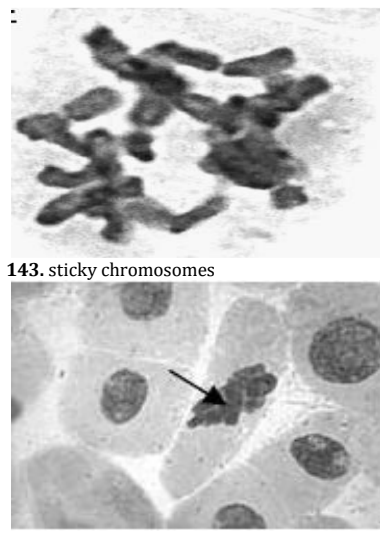

147. Sticky chromosomes

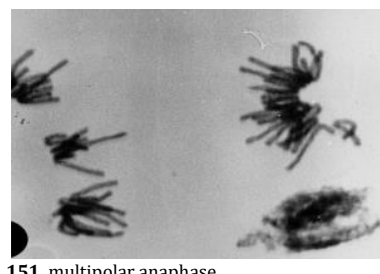

151. multipolar anaphase
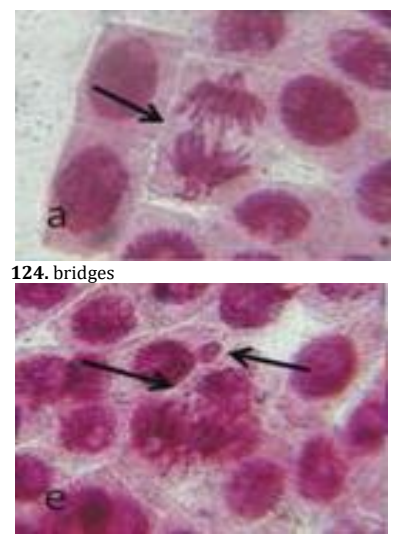

128. chromosome breaks and micronucleus

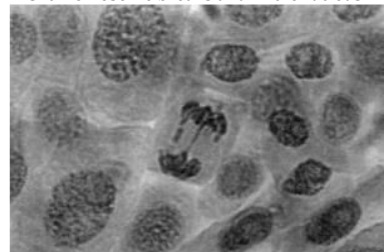

132. Late anaphase whit a bridge and

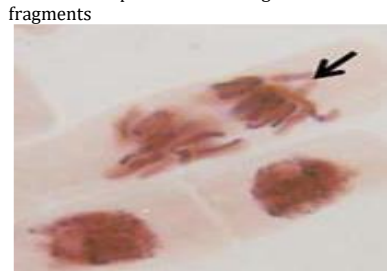

136. disturbed anaphase

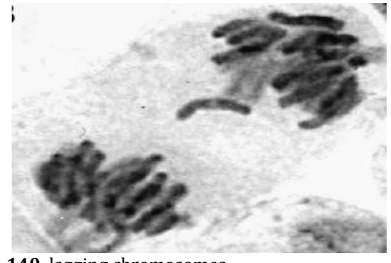

140. lagging chromosomes
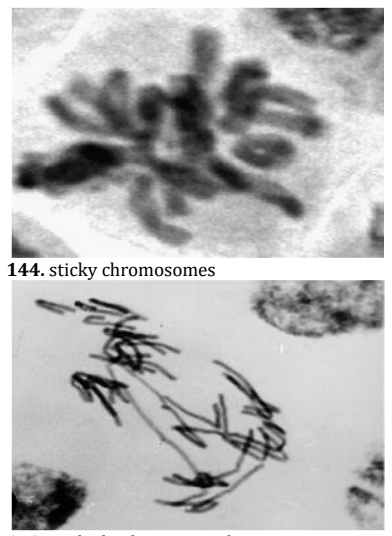

148. sticky bridges in anaphase

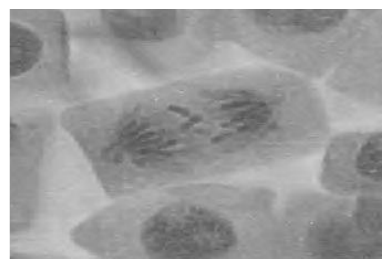

152. Anaphase with chromosome

fragments 


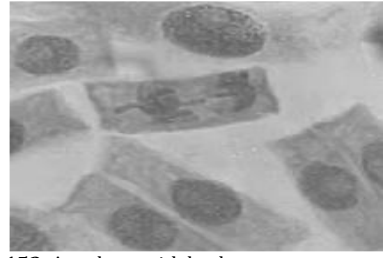

153. Anaphase with broken chromosome

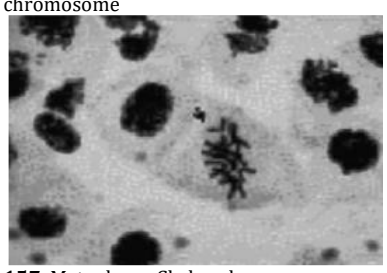

157. Metaphase: $\mathrm{Ch}$. break

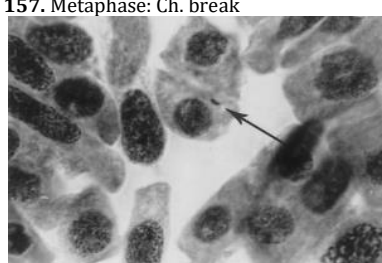

161. Prophase: Ch. Fragment

Fig. 2: Continue...

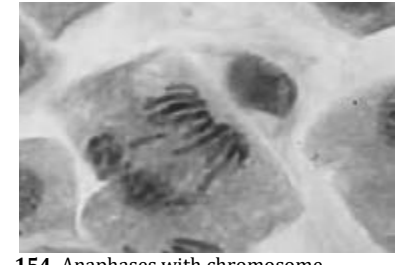

154. Anaphases with chromosome fragment

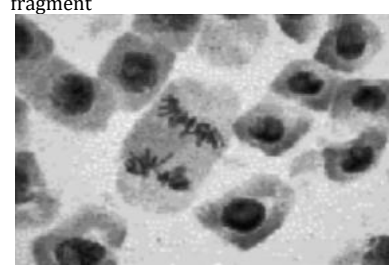

158. tripolar anaphase

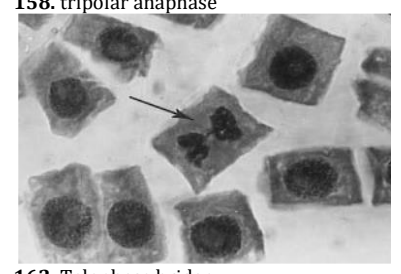

162. Telophase bridge

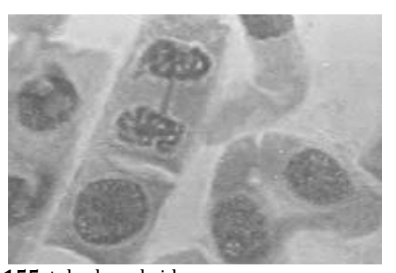

155. telophase bridge

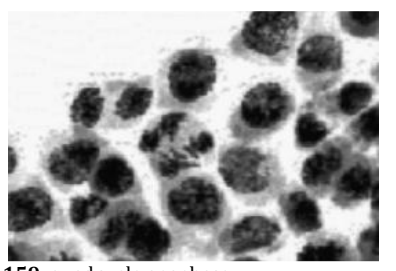

159. quadruple anaphase

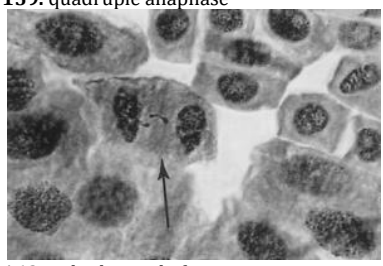

163. Telophase: ch. fragments

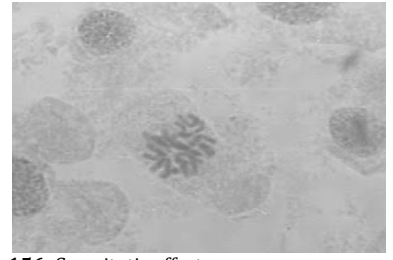

156. C - mitotic effect

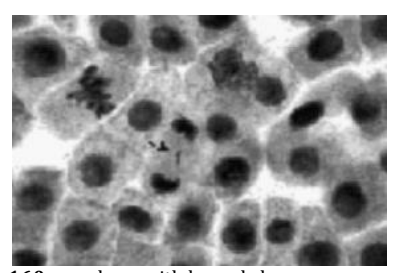

160. anaphase with lagged chromosome

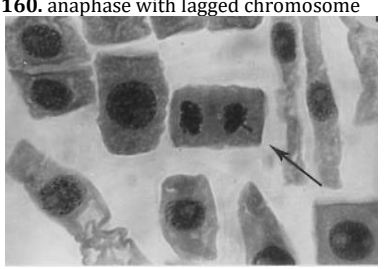

164. Telophase: vagrant $\mathrm{Ch}$.
The MN endpoint in A. cepa test is simple and easy to evaluate as compared to the NA and CA, which results from damaging and wrongly repaired cells. As a result of $\mathrm{MN}$ abnormality, the nucleus size in daughter cell is reduced as compared to parent cell. The MN size can be an effective parameter to assess the clastogenic and aneugenic effects in $A$. сера, since this species present a symmetric karyotype, which is homogeneous in relation to chromosomal size, with large and few chromosomes. Therefore, large MN would indicate an aneugenic effect resulting from a chromosome loss, whereas small MN may indicate a clastogenic action resulting from chromosome break. However, other cytogenetic techniques, such as chromosome banding and in situ hybridization, should be applied to make the analysis more reliable and accurate (Leme and Marin-Morales, 2008, 2009).

The MN abnormality is often results from the acentric fragments (clastogenic action) or lagging chromosomes or even from malfunctioning of the spindle/ malformation of the mitotic fuse (aneugenic action) that fail to incorporate into either of the daughter nuclei during telophase of the mitotic cells and can cause cell death due to the deletion of primary genes (Andrade et al., 2008; Sudhakar et al., 2001). The chromosome breakage are also associated with the formation of chromosome fragments and MN cells (Fiskesjo, 1993). Furthermore, the formation of MN may also result from spindle poisoning which is an anomalous disjunction of chromosomes at anaphase stage (Chandra et al., 2005; Grover and Kaur, 1999) or by eliminating the amplified genetic material (Mazzeo et al., 2011a). In general, the induction of $\mathrm{MN}$ in root meristems is the manifestation of chromosome breakage and disturbance of the mitotic process due to spindle abnormalities (Grover and Kaur, 1999) and
MN are considered an indication of a true mutation effect (Abdel Migid et al., 2007).

The formation of multinucleated cells may be the result of a preceding multipolar mitosis or the failure of cell plate formation, a cytokinesis disturbance (Abdel Migid et al., 2007). Lobate nuclei and polynucleated cells are resultant of $\mathrm{CA}$, as a consequence of multipolar anaphases, which are associated or not with chromosomal adherence, making the cells inviable (Fernandes et al., 2007b). Micronucleated cells can also arise from nuclear buds, which are eliminated from the nucleus through an active process during the $S$ phase (synthesis phase- stage of cell cycle in which DNA is replicated) of the cell cycle (Shimizu et al., 1998).

The clastogenic/aneugenic effects of toxic agents can be evaluated precisely on the basis of MN. The MN derived from clastogenic agents are reported to be smaller than $\mathrm{MN}$ derived from aneugenic action of toxic agents (Leme and Marin-Morales, 2008). The nuclear buds are indicative of an initial process of releasing the exceeding nuclear material and, consequently, they can also be related to the $\mathrm{MN}$ formation, which might be further eliminated from cytoplasm as mini cell (Fernandes et al., 2007b).

The MN due to induction of chromosomal breaks during cell division, as well as by the expulsion of excess genetic material by nuclear buds, which later eliminated as MN has also been reported (Mazzeo et al., 2011a) and this induction is correlated with inhibitory action of the enzyme topoisomerase II, responsible for the reconnection of fragments of DNA during the replication process, which results in the clastogenic effect of toxic agent (Mondrala and Eastmond, 2010; Whysner et al., 2004). Moreover, Alberts et al. (Alberts et al., 2008) affirm that the inhibition of topoisomerase II leads to a tangle of the daughter chromosomes, which remain intertwined and therefore 
unable to separate after replication. Thus, if such damage is not repaired, replication of a damaged DNA may lead to the amplification of the genetic material and extra material is originated by amplification and eliminated from the nucleus as an MN (Fernandes et al., 2007b; Mazzeo et al., 2011a). In view of $\mathrm{MN}$ importance, various authors used it as genotoxicity indicator for different systems (da Costa Machado Matos Carvalho et al., 2011; Geras'kin et al., 2011; Hoshina and Marin-Morales, 2009; Junior et al., 2007; Kwasniewska et al., 2012; Leme et al., 2008; Leme and MarinMorales, 2008; Marcano et al., 2004; Mazzeo et al., 2011a; Misik et al., 2011; Nunes et al., 2011; Smaka-Kincl et al., 1996; Tabrez and Ahmad, 2011).

Fig. 4 shows the MN's that are reported in literature in the $A$. сера root after exposure to various toxic agents, while percentage effect on $\mathrm{MN}$ formation versus negatice control can be seen in Table 1 .

\section{Industrial wastewater nature}

Industrial effluents are characterized by changes in turbidity, conductivity, total suspended solids (TSS), chemical oxygen demand (COD), biological oxygen demand (BOD) and total hardness (TH). Some industries are contaminating water bodies by toxic materials, while other are responsible for colored effluents (KANU and Achi, 2011). Beside, heavy metals, the industrial wastewater also contains a considerable amount of inorganic materials, organic compounds, salts, detergents, surfactants, dyes, food additives etc, which affect the water physico-chemical properties and toxicity to living organisms (Abbasi et al., 2016; Asadi et al., 2016; Balapure et al., 2016; Barbosa et al., 2014; Barrera-Díaz et al., 2014; Behbahani et al., 2016; Bertani et al., 2016; Bordes et al., 2015; Camacho-Muñoz et al., 2014; Carbajo et al., 2015; Chae et al., 2016; Cheballah et al., 2015; Chong et al., 2015; Chou et al., 2014; da Costa Filho et al., 2016; da Costa et al., 2012b; Expósito et al., 2016; Gatsios et al., 2015; Gatta et al., 2015; Goh et al., 2015; Hoshina and Marin-Morales, 2009; Hu et al., 2015; Huang et al., 2016; Jeon, 2015; Kang et al., 2016; Kuroda et al., 2015; Lee et al., 2016; Lee et al., 2015; Liu et al., 2016; Lovatel et al., 2015; Meneses-Jácome et al., 2016; Olorunfemi et al., 2011b; Pereyra et al., 2015; Polat et al., 2015; Prieto et al., 2015; Sarkar et al., 2015; Sasmaz and Yaman, 2008; Sasmaz et al., 2015a; Sasmaz et al., 2016b; Sasmaz et al., 2016c; Sasmaz et al., 2016d; Sasmaz et al., 2015b; Şaşmaz et al., 2011; Seow and Hauser, 2016; Singh et al., 2016; Sueviriyapan et al., 2016; Tamis et al., 2014; Vasiliadou et al., 2016; Vymazal, 2014; Zakharov and Bondareva, 2015; Zarpelon et al., 2016; Zheng et al., 2015).

The current contamination of water resources, especially as a consequence of anthropogenic discharges, is becoming a major problem in urban regions and changes in water chemistry obviously have deleterious effects on the living organisms inhabiting these areas, especially aquatic biota directly and humans beings indirectly (Adesola et al., 2016; Amadi and Ukpaka, 2015; Babarinde and Onyiaocha, 2016; Iqbal and Khera, 2015; Jafarinejad, 2016a; Jafarinejad, 2016b; Jamal et al., 2015; Majolagbe et al., 2016b; Ohe et al., 2004; Pandey et al., 2016a, b; Peter and Chinedu, 2016a; Qureshi et al., 2015b; Sayed, 2015; Shindy, 2016; Ukpaka, 2016b; Ukpaka et al., 2015; Ukpaka, 2016e, f; Ukpaka, 2016g; Ukpaka and Collins, 2016; Ukpaka, 2016h). Among the lethal and sub-lethal effects of the complex mixtures, fertility disorders as well as cellular, metabolic, and DNA damages are reported (Vianna Villela et al., 2007). Epidemiological studies have failed to clearly characterize these effects, since the experimental procedure to demonstrate this influence is a time-consuming task. This scenario stresses the importance of efficiently and continuously monitoring possible impacted areas using screening assays. The $A$. cepa test has been used to detect a variety of environmental pollutants in industrial wastewater and the results obtained were reported significant versus control (Leme and Marin-Morales, 2009). A number of studies have been performed using A. cepa test system and positive responses of A. cepa have been reported i.e., complex mixture (Aguiar et al., 2016; Andrade-Vieira et al., 2012; Blagojević et al., 2009; Chakraborty and Mukherjee, 2010; Chakraborty et al., 2009; Klauck et al., 2013; Mazzeo et al., 2011b; Pathiratne et al., 2015; Pekol et al., 2012; Singh et al., 2014; Vidaković et al., 1993; Yahaya et al., 2012), antitoxic agent (Aslantürk and Çelik, 2006; Frescura et al., 2012; Kuhn et al., 2015; Kumari et al., 2013; Majewska et al., 2003; Nantes et al., 2014; Oyeyemi and Bakare, 2013; Santana et al., 2016; Ślusarczyk et al., 2014; Ślusarczyk et al., 2016; Sultan and Çelik, 2009), contaminated soil (Christofoletti et al., 2013; Dash et al., 1988; Kataeva et al., 2012; Kopliku and Mesi, 2012b; Masood and Malik, 2013a; Meier et al., 1997; Oberholster et al., 2008; Paul et al., 2013; Saxena et al., 2004; Sayles et al., 1999), drugs (Firbas and Amon, 2014; Grisolia and Takahashi, 1991; Jos et al., 2003; Lutterbeck et al., 2015; Mišík et al., 2014; Onwuamah et al., 2014), detergents and surfactants (Bellani et al., 1991; Iqbal and Bhatti, 2015a; Pedrazzani et al., 2012; WANG and WANG, 2007), hair and food additive (Adeyemo and Farinmade, 2016; De Lima et al., 2010; Maiti et al., 2016; Onyemaobi et al., 2012; Roychoudhury and Giri, 1989; Tripathy and Rao, 2015), industrial wastewater (Bakare et al., 2012; BohórquezEcheverry et al., 2012; Chaparro and Pires, 2011; Gupta and Ahmad, 2012b; Gupta et al., 2012; Matsumoto and MarinMorales, 2004; Obute et al., 2005; Watharkar and Jadhav, 2014; Xing et al., 1995), leachate/sludge/sewage (Amin, 2002; Bakare, 2001; Bakare et al., 2012; Christofoletti et al., 2012; Corrêa Martins et al., 2016; Garaj-Vrhovac et al., 2013; Klauck et al., 2015; Magdaleno et al., 2008; Mazzeo et al., 2015; Obidoska and Jasińska, 2008; Pelegrini et al., 2007; Roig et al., 2012; Shashank and Suresh, 2013; Tanti et al., 2009), metallic compounds (Gömürgen, 2005; Meng and Zhang, 1992; RENCÜZOĞULLARI et al., 2001; Teerarak et al., 2009; Wierzbicka, 1994; Yi and Meng, 2003; Yıldız et al., 2009), metals (Arambašić et al., 1995; Arya and Mukherjee, 2014; Barbosa et al., 2010; Carruyo et al., 2008; Chang et al., 1997; Geremias et al., 2010; Hemachandra and Pathiratne, 2015; Jiang et al., 2014; Karaismailoglu, 2014; Kopliku and Mesi, 2012a; Kumar et al., 2015; Lerda, 1992; Liman, 2013; 
Liu et al., 2003; Liu et al., 1994; Marcano et al., 2002; Marcano et al., 2006; Md Nazmul et al., 2013; Mohan Murali Achary et al., 2008; Pal and Nandi, 1990; Palacio et al., 2005; Panda et al., 1997; Patnaik et al., 2013; Pepper et al., 1988; Qin et al., 2010; Seth et al., 2008a; Sharma et al., 2012; Singh, 2015; Wierzbicka, 1999a; Wierzbicka, 1999b; Wu et al., 2010; Yi et al., 2007b), model compounds (Abdel-Azeem, 2014; Al et al., 2013; Arkhipchuk et al., 2004; Asgher and Iqbal, 2013; Asita and Matobole, 2010; Ateeq et al., 2002; Bhatta and Sakya, 2009; BOKHARI et al., 2015; Cabaravdic, 2010; De Marco et al., 1986; Evandri et al., 2003; Flores Navarro et al., 2015; Freire et al., 2014; Geremias et al., 2011; González et al., 2012; Goujon et al., 2015; Hardy et al., 2011; Hassan and YASSEIN, 2016; Herrero et al., 2012; Jadhav et al., 2012a; Jadhav et al., 2012b; Jha et al., 2009; Jos et al., 2005; LIU et al., 2009; Mellado-García et al., 2016; Mesi and Kopliku, 2014; Mohammed et al., 2015; Pandey et al., 2014; Panneerselvam et al., 2012; Patil and Jadhav, 2013; Patra et al., 2005; Paul et al., 2013; Petriccione et al., 2013; Poonkuzhali et al., 2011a; Quilang et al., 2008; Ragunathan and Panneerselvam, 2007; Rainho et al., 2010; Ramos de Rainho et al., 2013; Rank et al., 2002; Rathore and Choubey, 2005; Ronchi et al., 1986; Tabrez and Ahmad, 2012; Trushin et al., 2013a; Trushin et al., 2013b; Türkoğlu, 2008; Wasi et al., 2011; Yi and Meng, 2003; Younis et al., 1989), disinfected water/tap water (Crebelli et al., 2002; Olorunfemi, 2013), nano materials (Babu et al., 2008; de Andrade et al., 2014; Fernández Freire et al., 2015; Ghodake et al., 2011; Ghosh et al., 2015; Grillo et al., 2015; Klančnik et al., 2011; Kumari et al., 2011; Kumari et al., 2009a; Nagaonkar et al., 2015; Pakrashi et al., 2014; Pesnya, 2013), pharmaceutical, pesticides, herbicides, fungicide \& insecticide (Akintonwa et al., 2009; Andrioli et al., 2006; Asita and Makhalemele, 2008; Bakare et al., 2009; Bianchi et al., 2016; Bianchi et al., 2015; Bolle et al., 2004; Çavuşoğlu et al., 2012a; Chauhan et al., 1986; Chauhan and Gupta, 2005b; da Lima et al., 2012; de Campos Ventura-Camargo and MarinMorales, 2016; Feretti et al., 2007; Fernandes et al., 2007a; Fisun and Rasgele, 2009; Goujon et al., 2014; İLBAȘ et al., 2012; Karaismailoglu, 2015; Kaymak and Muranli, 2005; Lateef et al., 2007; Liman et al., 2010b; Liman et al., 2011; Liman et al., 2015; Marin-Morales, 2015; Maselli et al., 2015; Mesi and Kopliku, 2013; Mishra et al., 2015; Mosuro et al., 1999; Musanovic et al., 2013; Mustafa and Suna Arikan, 2008; Ozakca and Silah, 2013; Özkara et al., 2015a; Özkara et al., 2015b; Palma et al., 2014; Rank and Nielsen, 1997a; Rodríguez et al., 2015; Saxena et al., 2010; Shaikh et al., 2012; Silveira et al., 2015; Sinha and Kumar, 2014; Srivastava and Mishra, 2009; Tripathy et al., 2013; Tripathy and Patel, 2015; Türkoğlu, 2012), plants extracts/vegetable grown in contaminated water and soil (Adegbite and Sanyaolu, 2009; Ali, 2010; Aşkin Çelik and Aslantürk, 2010; Bidau et al., 2004; Bittencourt De Souza et al., 2010; Bratu et al., 2012; Çavuşoğlu et al., 2016; Çavuşoğlu et al., 2012b; Chukwujekwu and Van Staden, 2014; Eren and Özata, 2014; Feretti et al., 2007; Frescura et al., 2012; Glińska et al., 2007; Glińska and Gabara, 2011; Jothy et al., 2013; Knoll et al., 2006b; Kuraś et al., 2006; Kwankua et al., 2010; Leite et al., 2015; Lubini et al.,
2008; Mauro et al., 2014; Mekki, 2014; Mendes et al., 2012; Mohammed et al., 2015; Olorunfemi et al., 2011a; Pastori et al., 2013; Prajitha and Thoppil, 2016; Ragunathan and Panneerselvam, 2007; Renjana and Thoppil, 2015; Saleh Alqasoumi, 2012; Sharma et al., 2011; Shibata and Hizume, 2002; Silva et al., 2013; Solanke, 2008; Soliman, 2001; Sondhi et al., 2008; Sultan and Celik, 2009; Tartar et al., 2006; Timothy et al., 2014; Yuet Ping et al., 2012), air pollution (Blagojević et al., 2009; Glasencnik et al., 2002; Glasenčnik et al., 2004), radioactive metals, radioactive wastes and radiations (Evseeva et al., 2005; Saghirzadeh et al., 2008; Tkalec et al., 2009; Udalova et al., 2014), surface water and contaminated river water (Athanásio et al., 2014; Barbério et al., 2011; da Costa et al., 2012a; de Oliveira Meneguetti et al., 2012; Düsman et al., 2014; Espinoza-Quiñones et al., 2009; Fawole et al., 2008; Fernandes et al., 2010; Geras'kin et al., 2011; Grippa et al., 2012; Júnior et al., 2007; Mazzeo and Marin-Morales, 2015; Mesi and Kopliku, 2011; Oberholster et al., 2008; Olorunfemi et al., 2012; Palma et al., 2010; Roy et al., 2015; Serra de Lima Moraes and Jordao, 2001; Vujošević et al., 2008; Wängberg et al., 1995), treated waste and wastewater (Ameta et al., 2003; Balan et al., 2013; Balan et al., 2012; Bhat et al., 2014; Brkanac et al., 2014; Edemaa and Okungbowa, 2011; Geremias et al., 2012; GRISALES PENAGOS et al., 2012; Gupta and Ahmad, 2012b; Iqbal et al., 2015a; Iqbal et al., 2014; Iqbal and Nisar, 2015; Jadhav et al., 2015; Kern et al., 2013; Kusumaningrum et al., 2012; Machado et al., 2012; Masood and Malik, 2013b; Mazzeo et al., 2011b; Netto et al., 2013; Olorunfemi et al., 2011c; Olorunfemi et al., 2013; Papa et al., 2016; Patel et al., 2015; Patil and Jadhav, 2013; Poonkuzhali et al., 2013; Poonkuzhali et al., 2011a; Prasad et al., 2013; Ravindran et al., 2015; Roa et al., 2012; Satar and Husain, 2011; Sobral et al., 2013; Viana et al., 2014; Wasi et al., 2011; Watharkar and Jadhav, 2014; Watharkar et al., 2013), toxin (Laughinghouse Iv et al., 2012), wastewater (Chaparro and Pires, 2011; Defaveri et al., 2009; Fatima and Ahmad, 2005, 2006a; Frank and Harangozó, 1994; Gupta and Ahmad, 2012b; Gupta et al., 2012; Kern et al., 2015; Kopliku et al., 2012; Magdaleno et al., 2014; Somashekar and Arekal, 1983; Tabet et al., 2015; Viana et al., 2014), complex environmental mixtures, solid waste, surface and ground water of an urban and industrial origin (SmakaKincl et al., 1996; Umebese et al., 2013), sludge from municipal treatment stations (Rank and Nielsen, 1998), wastewater contaminated soil (Kwasniewska et al., 2012), water bodies contamination with radioactive material (Evseeva et al., 2003), hospital wastewater (Guzy et al., 2012) and river water receiving industrial wastewater (da Costa Machado Matos Carvalho et al., 2011). In all these studies, author's used $A$. cepa test for toxicity monitoring and positive results have been documented.

In following section, the wastewaters and related matrices genetotoxic, cytotoxic and mutatoxic effect in $A$. cepa cells are discussed.

Application of $A$. cepa to detect the toxicity of industrial wastewater and contaminated soil and river water 
Table 1: The chromosomal aberrations, nuclear aberrations, aberrant cell, total aberration, micronucli, mitotic index and root length percentage effect observed in A. cepa root cells exposed to different toxic agents

\begin{tabular}{|c|c|c|c|c|c|c|}
\hline Industry & RL & MI & MN & $\mathrm{AC} / \mathrm{TA}$ & CA and NA types & Refeences \\
\hline $\begin{array}{l}\text { Metal industry leachates } \\
(10 \%)\end{array}$ & --- & --- & $0.14 \% *$ & --- & $\begin{array}{l}\text { Multipolar ana, Aberrant anaphase, unequal chromosome, } \\
\text { bridge (4\%), stikiness(83.33\%), Frag(10\%), Lagg(80\%) }\end{array}$ & (Chandra et al., 2005) \\
\hline Dye industry leachates & --- & --- & $7.81 \%$ & $/ 0.13 \%$ & $\begin{array}{l}\text { Multipolar anae, aberrant anae, unequal chromosome, } \\
\text { stikiness and lagg }\end{array}$ & (Chandra et al., 2005) \\
\hline Paint industry WW & --- & --- & --- & $3-7 \% /$ & Bridges(3-5\%), stikiness(6\%) and Frag(3-5\%) & (Samuel et al., 2010) \\
\hline Textile WW & --- & --- & --- & $4-5 \% /$ & Bridges(2-5\%), Stikiness(1-2\%) and Frag(2-5\%) & \\
\hline Bottling industry $(10 \%)$ & --- & --- & $1 \%$ & $5-8 \% /$ & Bridges(4-6\%), Stikiness(5-7\%), Frag(2-3\%) and lagg(1-3\%) & (Olorunfemi et al., 2011) \\
\hline Rubber industry (10\%) & --- & --- & $1-5 \%$ & $2-8 \% /$ & Bridges(3-5\%), Stikiness(1-7\%), Frag(1-4\%) and $\operatorname{lagg(1-5\% )}$ & (Olorunfemi et al., 2011) \\
\hline Brewery $(10 \%)$ & --- & --- & $1 \%$ & $5 \% /$ & Bridges(2-5\%), Stikiness(1-4\%), Frag(1-2\%) and lagg(1-4\%) & (Olorunfemi et al., 2011) \\
\hline Chemical Industry (100\%) & --- & --- & --- & $48 \% /$ & Abn. Pro(14\%), Meta(ND), Ana(100\%) and Telo(ND) & $\begin{array}{l}\text { (Abu and Ezeugwu, } \\
\text { 2008) }\end{array}$ \\
\hline Al metal leachates $(5 \%)$ & --- & --- & --- & --- & $\begin{array}{l}\text { Cell death (73-90\%), C-mitosis (28-33\%), multipolar ana } \\
(0.08-0.13 \%) \text {, Bridge( } 25.12 \%) \text {, Stikiness(5.08\%) and } \\
\text { frag( } 8.33 \%)\end{array}$ & (Andrade et al., 2008) \\
\hline \multirow[t]{2}{*}{ Industrial area site } & --- & $-62 \%$ & $52.00 \%$ & $/ 71 \%$ & $\begin{array}{l}\text { Bridges, ch. rings, frag, late separation, disturbed ch, prophase } \\
(-24 \%) \text {, meta( }(-23 \%) \text {, ana( }(-36 \%) \text { and telo( }(-26 \%)\end{array}$ & (El-Shahaby et al., 2003) \\
\hline & --- & --- & --- & $35.47 \% /$ & Bridges and stikiness, & $\begin{array}{l}\text { (Abdel Migid et al., } \\
\text { 2007) }\end{array}$ \\
\hline River water summer & --- & --- & $58.06 \%$ & --- & CA(30.91\%), mitotic cell $(-37.90 \%)$ and cell death $(40.74 \%)$ & (Bianchi et al., 2011) \\
\hline River water autumn & --- & --- & $29 \%$ & --- & $\mathrm{CA}(45.18 \%)$ and mitotic cell $(-36.22 \%)$ & (Bianchi et al., 2011) \\
\hline River water winter & --- & --- & $38 \%$ & --- & CA(11.09\%), mitotic cell $(-57 \%)$ and cell death(78\%) & (Bianchi et al., 2011) \\
\hline $\begin{array}{l}\text { Soil sample irrigated with } \\
\text { industrial WW }\end{array}$ & --- & --- & $53-81 \%$ & --- & & $\begin{array}{l}\text { (Cabrera and Rodriguez, } \\
\text { 1999) }\end{array}$ \\
\hline Textile WW (100\%) & --- & --- & $5.00 \%$ & --- & $\begin{array}{l}\text { Ana bridge, ana loss,,microcyte, meta loss, C-meta, delayed } \\
\text { telo, telo bridge,binucleated cell, adherence and cell death }\end{array}$ & $\begin{array}{l}\text { (Carita and Marin- } \\
\text { Morales, 2008) }\end{array}$ \\
\hline $\begin{array}{l}\text { Industrial WW } \\
\text { contaminated soil }\end{array}$ & --- & $-30 \%$ & $\begin{array}{l}0.2- \\
23 \%\end{array}$ & --- & & (Cotelle et al., 1999) \\
\hline Lemon juice factory & $-27 \%$ & --- & --- & --- & Aberrant ana & (Gana et al., 2008) \\
\hline Paper factory & $-16 \%$ & $-33 \%$ & $1 \%$ & --- & Aberrant ana & (Gana et al., 2008) \\
\hline Mining industry & $-14 \%$ & $-46 \%$ & $1.60 \%$ & --- & Aberrant ana & (Gana et al., 2008) \\
\hline Sugar industry & $-14 \%$ & $-46 \%$ & --- & --- & Aberrant ana & (Gana et al., 2008) \\
\hline \multirow[t]{2}{*}{ Acid mine drainage } & --- & & --- & --- & Chromatid breaks and fragmentation & (Geremias et al., 2012) \\
\hline & --- & $-53 \%$ & + & $/ 70 \%$ & Lagging chromosme, bridges and Stikiness & (Grisolia et al., 2005) \\
\hline Textile and paper mills & --- & --- & $65 \%$ & --- & Aberant ana & (Grover and Kaur, 1999) \\
\hline Refinery WW & $-68 \%$ & --- & $90 \%$ & $/ 97 \%$ & $\begin{array}{l}\text { Laggard chromosome }(88 \%) \text {, late anap, bridges, stikiness and } \\
\text { frag }(93 \%) \text {, }\end{array}$ & $\begin{array}{l}\text { (Gupta and Ahmad, } \\
\text { 2012) }\end{array}$ \\
\hline $\begin{array}{l}\text { Petroleum refinery } \\
\text { contaminated river water }\end{array}$ & --- & $-41 \%$ & --- & --- & Cell death and CA's(88.88\%) & $\begin{array}{l}\text { (Hoshina and Marin- } \\
\text { Morales, 2009) }\end{array}$ \\
\hline Textile WW (30\%) & $-6 \%$ & $-19 \%$ & --- & $/ 86 \%$ & $\begin{array}{l}\text { Chromosome breaks, delayed ana, multipolar ch, binucleated } \\
\text { cell, ana with loss, meta with loss, bridges(92\%) and lagg(7\%) }\end{array}$ & (Jadhav et al., 2010) \\
\hline Tannery waste summer & $-71 \%$ & $-9 \%$ & --- & --- & Overall microscopical effects(20\%) & (Junior et al., 2007) \\
\hline Tannery waste winter & $-37 \%$ & $-28 \%$ & --- & --- & Overall microscopical effects(11\%) & (Junior et al., 2007) \\
\hline Pesticide contaminated soil & --- & --- & --- & --- & CA, bridges(40\%) and Frag(14\%) & (Kong and Ma, 1999) \\
\hline $\begin{array}{l}\text { Soil sample (Chernobyl } \\
\text { accident) }\end{array}$ & --- & -- & -- & $/ 81.02 \%$ & $\begin{array}{l}\text { Multipolar ana(95.65\%), C-mitosis, vagrant } \operatorname{ch}(84.21 \%) \text {, } \\
\text { bridges(29.52\%), stikiness and frag(12.53\%) }\end{array}$ & (Kovalchuk et al., 1998) \\
\hline $\begin{array}{l}\text { Oil refinery contaminated } \\
\text { river water }\end{array}$ & --- & --- & --- & --- & $\begin{array}{l}\text { Lobated nuclei, polynucleated cells, nuclear buds, mini cells, C- } \\
\text { metaphases(78\%), multipolarity(95\%), Ch. losses (96\%), } \\
\text { Lagg(90\%), bridges(73\%), stikiness(14.89\%) and frag(80\%) }\end{array}$ & (Leme et al., 2008) \\
\hline Chemical industry & --- & --- & --- & --- & $\begin{array}{l}\text { CA and interphase nucleus aberrations, multinucleated cells, } \\
\text { late-separating ch, unoriented ch. bridges, stikiness, lagg }\end{array}$ & $\begin{array}{l}\text { (Abdel Migid et al., } \\
\text { 2007) }\end{array}$ \\
\hline Sewage $(20 \%)$ & $-7.50 \%$ & $-41 \%$ & --- & --- & $\begin{array}{l}\text { Stickiness }(\text {-mitncis }(1 \%) \text { vaorant }(4 \%) \text { and hridoes }(3 \%) \\
15\end{array}$ & (Ukaegbu and Odeigah, \\
\hline
\end{tabular}


Table 1: Continue...

\begin{tabular}{|c|c|c|c|c|c|c|c|c|}
\hline Paper mill & & $-44.28 \%$ & & $-44 \%$ & --- & --- & $\begin{array}{l}\text { Early ana(7.7\%), stickiness(3.1\%), C- } \\
\text { mitosis(12.2\%), bridges and } \\
\text { fragments(6.8\%), banded ch(3.4\%) and } \\
\text { multipolar ana }\end{array}$ & (Tipirdamaz et al., 2003) \\
\hline $\begin{array}{l}\text { Disinfected water with } \\
\mathrm{ClO}_{2}\end{array}$ & --- & & & $-10 \%$ & --- & --- & Anaphase aberation & (Monarca et al., 2003) \\
\hline $\begin{array}{l}\text { Disinfected water with } \\
\mathrm{NaClO}\end{array}$ & --- & & & $-11 \%$ & -- & --- & Anaphase aberation & (Monarca et al., 2003) \\
\hline $\begin{array}{l}\text { Disinfected water with } \\
\text { PAA }\end{array}$ & --- & & & $7.40 \%$ & --- & --- & Anaphase aberation & (Monarca et al., 2003) \\
\hline $\begin{array}{l}\text { Gasoline station WW } \\
(50 \%)\end{array}$ & --- & & & $-14 \%$ & --- & $60 \% /$ & Fragments and laggard chromosomes & $\begin{array}{l}\text { (Oliveira-Martins and Grisolia, } \\
\text { 2009) }\end{array}$ \\
\hline $\begin{array}{l}\text { Industrial water } \\
\text { contaminated soil } \\
\text { extracts }\end{array}$ & --- & & --- & & --- & --- & $\begin{array}{l}\text { Fragments, bridges, lagg, vagrant and } \\
\text { Aberrant ana(92.13\%) }\end{array}$ & (Katnoria et al., 2011) \\
\hline Textile effluents (100\%) & & $-92.82 \%$ & & $-40 \%$ & --- & $/ 6.8 \%$ & $\begin{array}{l}\text { Stickiness, vagrant, bridges, fragments } \\
\text { and multipolar anaphase }\end{array}$ & (Oriaku et al., 2011) \\
\hline $\begin{array}{l}\text { Insecticide and shoe } \\
\text { polish factory }(100 \%)\end{array}$ & $--\cdot$ & & & $-50 \%$ & --- & $81 \% /$ & $\begin{array}{l}\text { Abn. prophase }(94 \%) \text {, abn. meta }(72 \%) \text {, } \\
\text { abn. ana( } 85 \%) \text {, abn. telo( } 48 \% \text { for } 75 \% \\
\text { conc.) }\end{array}$ & (Abu and Ezeugwu, 2008) \\
\hline Pharmaceutical WW & & $-95 \%$ & & $-80 \%$ & --- & --- & C-mitosis, ana bridge, multiple nuclei & (Abu and Mba, 2011) \\
\hline $\begin{array}{l}\text { Al metal industry }(25 \\
\%)\end{array}$ & & $-98 \%$ & --- & & --- & --- & $\begin{array}{l}\text { Late seg( } 83 \%) \text {, bridges }(70 \%) \text {, } \\
\text { stickiness }(63.63 \%) \text {, frag, cell death } \\
(90 \%), \text { C-metaphase(33\%), multipolar } \\
\text { ana }\end{array}$ & (Andrade et al., 2008) \\
\hline River wastewater & & $-48 \%$ & & $-66 \%$ & $50 \%$ & --- & $\begin{array}{l}\text { Anaphase bridges, ch. fragments and } \\
\text { delayed anaphase }\end{array}$ & $\begin{array}{l}\text { (da Costa Machado Matos } \\
\text { Carvalho et al., 2011) }\end{array}$ \\
\hline Mineral water & --- & & --- & & --- & --- & Bridges, fragments, lagg and C-mitosis & (Evandri et al., 2000) \\
\hline $\begin{array}{l}\text { Radium production } \\
\text { industry territory water }\end{array}$ & --- & & & $-50 \%$ & & $/ 2-4 \%$ & $\begin{array}{l}\text { Single and double fragments, bridges } \\
\text { and vagrant ch. }\end{array}$ & (Evseeva et al., 2003) \\
\hline $\begin{array}{l}\text { Landfill leachates- } \\
\text { drainage pumping } \\
\text { stations }\end{array}$ & --- & & & $-80 \%$ & --- & --- & $\mathrm{CA}$ & (Kwasniewska et al., 2012) \\
\hline $\begin{array}{l}\text { Refinery contaminated } \\
\text { river WW }\end{array}$ & --- & & & $-13 \%$ & $\begin{array}{l}95 \% \& \\
98 \%(F 1)\end{array}$ & --- & CA $(89 \%)$ & (Leme and Marin-Morales, 2008) \\
\hline HC mixture & --- & & --- & & $85 \%$ & $/ 79 \%$ & $\begin{array}{l}\text { Adherence, lagg, C-mitosis, Ch. loss, } \\
\text { bridges, Ch. breaks, multipolarity, } \\
\text { nuclear buds, polynucleated, polypolid, } \\
\text { mini cell, lobulated }\end{array}$ & (Mazzeo et al., 2011) \\
\hline Disinfected water & --- & & --- & & $4 \%$ & --- & CA $(3.02-4.14 \%)$ & (Monarca et al., 2002) \\
\hline Oil field WW & --- & & & $-71 \%$ & & $/ 90 \%$ & $\begin{array}{l}\text { Stickiness, C-mitosis, vagrant, } \\
\text { multipolar ana, bridges and frag }\end{array}$ & (Odeigah et al., 1997) \\
\hline Bottling industry (10\%) & & $-33 \%$ & --- & & -- & $7.72 \% /$ & $\begin{array}{l}\text { Binucleated cell, lagg, bridges, frag, } \\
\text { stickiness, vagrant, C-mitosis and } \\
\text { disturbed spindle }\end{array}$ & (Olorunfemi et al., 2011) \\
\hline Rubber industry (10\%) & & $-21 \%$ & --- & & --- & $7.33 \% /$ & $\begin{array}{l}\text { Binucleated cell, lagg, bridges, frag, } \\
\text { stickiness, vagrant and disturbed } \\
\text { spindle }\end{array}$ & (Olorunfemi et al., 2011) \\
\hline $\begin{array}{l}\text { Brewery industry } \\
(10 \%)\end{array}$ & & $-13 \%$ & --- & & --- & $5.41 \% /$ & $\begin{array}{l}\text { Binucleated cell, lagg, bridges, frag, } \\
\text { stickiness, vagrant, C-mitosis, disturbed } \\
\text { spindle }\end{array}$ & (Olorunfemi et al., 2011) \\
\hline $\begin{array}{l}\text { WW phosphoric } \\
\text { gypsum plant }\end{array}$ & & $-73 \%$ & --- & & -- & $/ 93 \%$ & $\begin{array}{l}\text { C-mitosis, multipolar ana, lagg ch, sticky } \\
\text { and polyploidy }\end{array}$ & (Pavlica et al., 2000) \\
\hline Textile WW & --- & & & $-10 \%$ & --- & $/ 87 \%$ & Bridges, frag and vagrant & (Phugare et al., 2011) \\
\hline $\begin{array}{l}\text { Treated WW } \\
\text { (bioremediation) }\end{array}$ & --- & & & $-1 \%$ & --- & $/ 55 \%$ & & (Phugare et al., 2011) \\
\hline WW treatment plants & --- & & --- & & --- & $/ 45 \%$ & $\begin{array}{l}\text { Bridges }(31 \%) \text {, frag }(66 \%) \text {, } \\
\text { vagrant }(66 \%) \text {, other berations }(66 \%)\end{array}$ & (Rank and Nielsen, 1998) \\
\hline Oil refinery $(100 \%)$ & --- & & & $-4 \%$ & $25 \%$ & & & (Rodrigues et al., 2010) \\
\hline
\end{tabular}


Table 1: Continue...

\begin{tabular}{lllllll}
\hline Industrial mixture & & -- & & & & $\begin{array}{l}\text { CA (polar slip, irregularities at } \\
\text { anaphase, irregular distribution of ch in } \\
\text { meta, Ch. distortion, lagg, stickiness and } \\
\text { bridges) } \\
\text { Adherence, breaks, bridges, nuclear }\end{array}$ \\
$\begin{array}{l}\text { Oil refinery landforming } \\
\text { Industrial waste }\end{array}$ & & $-50 \%$ & --- & & -- & \\
buds and other CA(36\%) \\
mixture
\end{tabular}

Fertilizer \& petrochemical WW $\begin{array}{llll}-30 \% & -- & -- & / 83 \%\end{array}$
Multipolar ana, anaphase bridge, ana frag, other aberrations (up to $93 \%$ )
(Sik et al., 2009)

(Souza et al., 2009)

(Tabrez and Ahmad, 2011)

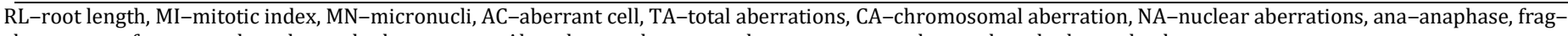

\section{Petroleum and petrochemical}

Petroleum industry is one of the most important sources of energy, and waste water discharged is a serious problem from environmental point of view. The refinery effluents consist of compounds from original crude oil stock as well as metallic ( $\mathrm{Zn}, \mathrm{Cr}, \mathrm{Ni}, \mathrm{Pb}, \mathrm{Cu}$ ) and non-metallic constituents. Phenols are also a major component of refinery wastewater. Moreover, among the hydrocarbons present in crude oil, the polycyclic aromatic hydrocarbons (PAHs) are among the most dangerous environmental contaminants due to their toxicity, carcinogenicity and mutagenicity (Gupta and Ahmad, 2012a). According to Leme and Marin-Morales (Leme and Marin-Morales, 2009), aromatic hydrocarbons are considered hazardous environmental pollutants due to the harmful effects they have on different living organisms.

The genotoxicity of petroleum refinery wastewater was investigated using $A$. cepa assays. Bulbs were germinated in distilled water and exposed to $20 \%, 40 \%$. $60 \%, 80 \%$ and $100 \%$ wastewater for $48 \mathrm{~h}$. Root analysis revealed that the wastewater induced mitodepressive effects in dose dependent manner, culminating in total mitotic inhibition at $100 \% \mathrm{v} / \mathrm{v}$ concentration. Among CA's, stickiness, erosion of chromatin, vagrant chromosomes, fragments and anaphase bridges were observed in root oxposed to $60 \%$ $80 \%$ of wastewater. Lower concentrations (20\%-40\%) induced c-mitosis as the major aberration (Obute et al., 2005).

The toxicity of river water impacted by an oil pipeline leak was studied (Leme and Marin-Morales, 2008) and the $A$. серa meristematic cell exposed to wastewater showed clastogenic and aneugenic effects. CA's inductions were most common, which were attributed to the presence of hydrocarbons. In another study, same authors also reported the CA's and MN induction in A. cepa roots when exposed to river water contaminated with petroleum hydrocarbons and polycyclic aromatic hydrocarbons (PAHs). The larger CA's and $\mathrm{MN}$ incidence in the meristematic cells of $A$. cepa was documented after exposure to water sample collected during the dry season, at the spring of the river, where the oil leak has arisen. The $F_{1}$ cells from roots exposed to such sample were also analyzed for the incidence of $\mathrm{MN}$, also showing a larger frequency of irregularities (Leme et al., 2008).

In order to assess the toxicity before and after landfarming bioremediation of oil refineries wastwater, $A$. cepa MN, NA and CA endpoints were studied (Souza et al., 2009). Authors investigated the landfarming and landfarming with rice hulls amendment before and after hydrocarbons biodegradation. Landfarming presented $13.5 \mathrm{~g} / \mathrm{kg}$ of total petroleum hydrocarbons which induced strong clastogenic and mutagenic effects in A. cepa root cells. After 108 days of biodegradation, the concentration of hydrocarbons decreased by $27 \%$ and there was significant reduction in mitotic abnormalities, CA, MN and nuclear bud. Landfarming treated with rice hulls decreased the $\mathrm{CA}, \mathrm{MN}$ and nuclear bud and correlated this efficiency with the biodegradation efficacy of landfarming. Finally, authors concluded that the $A$. cepa test is suitable to evaluate biodegradation process in soils contaminated by petroleum products. In another study, wastewater collected from petrochemical industry showed negative effect on the growth of $A$. cepa roots (CA's) for $24 \mathrm{~h}$ of exposure only (Nielsen and Rank, 1994). The MI, MN and CA's as indicators of toxicity in A. cepa exposed to petroleum wastewater were used and results showed that there was a significant alteration in $\mathrm{MI}$ and $\mathrm{MN}$ and CA's induction (Turkmen et al., 2009).

The toxicity of Mathura refinery wastewater adjoining area of Mathura refinery, Mathura, India was monitored using $A$. cepa roots. In exposed roots a significant formation of MN and CA's (bridges) was observed (Gupta and Ahmad, 2012a). The cytogentic effects of Mathura refinery wastewater and Aligarh wastewater of Northern India were also confirmed using $\mathrm{IC}_{50}$ value in $A$. cepa root. $A$. cepa genotoxicity test demonstrated a considerable amount of chromosomal damage. The aberration index for Aligarh wastewater and refinery wastewater was recorded to be $11.2 \%$ and $14.7 \%$, respectively, whereas the aquaguard mineral water serving as negative control displayed the aberration index value to be $2.6 \%$. The genotoxicity of both industrial wastewaters was reduced to a remarkable extent in presence of mannitol, the hydroxyl radical scavenger. Chromosomal aberrations were predominantly stickiness and stray chromosomes in case of Aligarh wastewater, while clumping and stickiness in case of refinery wastewater, thereby affirming the genotoxicity of both test waters (Fazili and Ahmad, 2014).

Similarly, the toxicity (root growth inhibition, malformation and CA's formation) of an oil field wastewater in the A. cepa root cells was reported (Odeigah et al., 1997). A 
series of 10 small bulbs of $A$. cepa were cultivated in various concentrations of the wastewater and after $48 \mathrm{~h}$ exposure, root tips were analyzed for cytological studies, while root lengths were measured after $96 \mathrm{~h}$, which resulted in significant dose dependent root growth inhibition. The wastewater treated group of plant showed significantly increased frequency of CA's such as sticky chromosomes, Cmitosis, spindle multipolarity, bridges and fragments. At lower concentrations only C-mitosis was observed.

While studying the nature of Santa Maria da Vitória River, Brazil (receive domestic, municipal and agricultural waste), Grippa et al. (2012) employed A. cepa test. Water samples were collected from 5 sites in the rainy season (March-high temperatures) and in the dry season (August-low temperatures) in 2006. The CA index was significantly higher at 2 sites in the rainy season compared with the dry season. This genotoxic effect may be related to agricultural influence and to domestic effluents that occur close to these 2 sites and to the presence of fertilizers, pesticides and iron oxide from the basin soils that are leached out by the rain and reach the river. In another study, the genotoxic potential of effluents from oil refineries by $A$. cepa test was studied (Rodrigues et al., 2010). The MN assay revealed the presence of $25 \% \mathrm{MN}$ and $4 \%$ of $\mathrm{MI}$ inhibition after $24 \mathrm{~h}$ of treatment, however, these values were found non-significant in comparison to control. In order to evaluate the genotoxicity of petrochemical wastewater in $A$. серa, CA test was employed (Vujosevic et al., 2001). In A. cepa root clear growth inhibition was observed. The CA abnormalities were also observed statistically significant in the treated group of plant. In another study, the toxicity and genotoxicity of wastewater from gasoline stations in Brasília, Brazil was studied by assessing CA's and MI in A. cepa root cells. The cell proliferation was significantly inhibited, especially at the highest concentrations. These results showed that although the effluent from gasoline stations was processed by an oil/water separation system before being discharged into the main sewage system, the wastewater still contained toxic compounds and further treatment was suggested to reduce the toxicity load (Oliveira-Martins and Grisolia, 2009).

Recently, the toxic effect of Nigerian Agip Oil Company (NAOC), Ogboinbiri, Bayelsa State was studied. Macroscopic analysis showed that A. cepa exposed to wastewater revealed concentration-dependent root growth inhibition with an $\mathrm{EC}_{50}$ value of $14.8 \%$ at 96 h. Root tip cells of the onion bulbs processed for cytological studies showed chromosomal aberrations at all concentrations. Along with aberrant chromosomes and reduction of MI was also observed in concentration-dependent manner and authors suggested the proper treatment of process water before its discharge into water bodies to avoid cyto-genetic damages to aquatic lives (Olorunfemi et al., 2015).

Similar to petroleum refinery effluents, Tanti et al. (2009) studied the toxicity of the oil refinery sludge using $A$. cepa assay. Roots exposed to different concentrations showed high MI values and lower percentage of abnormality in roots treated with diluted sludge. The MI values were decreased with concentrations. Binucleate, sticky anaphase erosion, C-metaphase, polyploidy, unequal distribution of chromosomes, spiral nature of chromosome and distortion of poles were the common CA's. Both duration and concentration of the treatment influenced the cell division. Results revealed that sludge generated by oil refinery may induce negative impact on crop and other biota in the agroecosystem. The petrochemicals, air liquid and polyester resin effluents using $A$. сера assay were also studied (Abu et al., 2015). Chemical analysis of the wastewaters showed high concentration of heavy metals. Mitodepressive were observed and MI vlues were recorded as 14.0, 5.3 and 4.1 in control, air liquid and polyester resin effluents, respectively. Percent abnormal dividing cells (C-mitotic effects to precocious chromosomes and anaphase bridges) increased with increase in wastewater concentration. Results indicates the cytotoxic and genotoxic effects of wastewaters tested, which could serve as indicator of the deleterious effects of wastewaters tested on living organisms at the point of discharge - either on land or water bodies. The need for sound sewerage system that would protect flora and fauna in the ecosystem was suggested.

The genotoxic effects of refined petroleum products (Petrol, Kerosene) has also been assessed using $A$. cepa test (Obute et al., 2016). The roots were treated with 10, 25 and $50 \%$ of these chemicals for 1,3 and $6 \mathrm{~h}$ and resultantly, depressive effects on mitosis i.e., reduced MI, mitotic phases abnormalities and CA's (anaphase bridges) were observed in petrol and zobo, laggards and vagrant chromosomes observed in zobo, while sticky chromosomes were observed in all sample tested. MN inductions were observed in zobo and petrol, whereas ghost cells were observed in zobo samples. Results suggested the presence of clastogens agents in tested samples and authors advised minimal consumption, contact and reduction in the duration of exposure.

The effect of oil refineries wastewater on $A$. серa roots is attributed to changes in the cellular volume and permeability of the cellular membrane, which seems to be associated with changes in the cytoskeleton. One reason for the increase of permeability would be due to lipophilic compounds that enhances the permeability and as a consequence the plasma membrane it loses the protection function (Hoshina and Marin-Morales, 2009; Thakkar et al., 2000). Some PAHs can induce an increase in the production of reactive oxygen species (ROS) and ROS can affect the cytoskeleton by changes in the redox state of regulatory enzymes and ultimately cell death, due to the exposition to petroleum by products such as aromatic hydrocarbons (Gomez-Mendikute and Cajaraville, 2003). In this regard, various authors reported the toxicity by exposing the A. cepa roots to aqueous solution of aromatic hydrocarbons (Rank and Nielsen, 1994). The genotoxic and mutagenic potential of benzene, toluene, ethylbenzene and xylene were also reported by applying CA and MN A. cepa test system (Mazzeo et al., 2011a).

\section{Metallurgical waste and metals}


Table 2: The observed toxicities in A. cepa root cells as a result of exposure to industrial waste, contaminated soil and river water

\begin{tabular}{|c|c|c|c|c|c|}
\hline Waste source & Physiochemical properties & Endpoint & $\begin{array}{l}\text { Cell } \\
\text { Type }\end{array}$ & Results & References \\
\hline $\begin{array}{l}\text { Engineering plant } \\
\text { WW }\end{array}$ & The wastewater contain heavy metals & & MC & $\mathrm{TC}$ & $\begin{array}{l}\text { (Frank and Harangoz, } \\
\text { 1994) }\end{array}$ \\
\hline Mixture & Metallic, chemical, textile, petrochemical, pulp and paper industry WW & $\mathrm{CA}, \mathrm{MI}$ & MC & GT, CT & $\begin{array}{l}\text { (Nielsen and Rank, } \\
\text { 1994) }\end{array}$ \\
\hline Mixture & Composition not reported & $\mathrm{MN}$ & $\begin{array}{l}\mathrm{MC} \& \\
\mathrm{~F}_{1}\end{array}$ & MT & (Ma et al., 1995) \\
\hline \multirow[t]{2}{*}{ Mixture } & $\begin{array}{l}\text { Industrial and municipal wastewater, biological treatment plant } \\
\text { output water; water from the Drava river }\end{array}$ & $\mathrm{CA}, \mathrm{MI}$ & MC & $\begin{array}{l}\text { CT, GT, } \\
\text { MT }\end{array}$ & $\begin{array}{l}\text { (Smaka-Kincl et al., } \\
\text { 1996) }\end{array}$ \\
\hline & & $\mathrm{MN}$ & & & \\
\hline Oil field wastewater & Composition not reported & CA, MI & MC & GT, CT & (Odeigah et al., 1997) \\
\hline $\begin{array}{l}\text { WW treatment } \\
\text { plants ludge }\end{array}$ & $\begin{array}{l}\text { pb-51-390, Ni-17-45, Cr-32-137, Zn-691-1420, Cu-82-378 and Cd-0.8- } \\
6.2 \mathrm{mg} / \mathrm{Kg}\end{array}$ & MI, CA & $\mathrm{MC}$ & CT, GT & $\begin{array}{l}\text { (Rank and Nielsen, } \\
\text { 1998) }\end{array}$ \\
\hline $\begin{array}{l}\text { Soil sample } \\
\text { (Chernobyl } \\
\text { accident) }\end{array}$ & $\begin{array}{l}\left.\mathrm{Cs}_{137}-2287, \mathrm{~K}_{40}-222\right), \mathrm{Th}_{232}-10 \text { and } \mathrm{Sr}_{90} \mathrm{~Bq} / \mathrm{Kg}, \mathrm{pH}-5.65, \mathrm{~N}-5.60, \mathrm{P}- \\
32.0, \mathrm{~K}-22.5 \text {, humus-3.63\%, Cu-2.2, } \mathrm{Zn}-27.2, \mathrm{Cd}-0.5, \mathrm{Cr}-0.52, \mathrm{Ni}-1.02 \\
\mathrm{~Pb}-15.32 \text { and } \mathrm{Hg}-0.008 \mathrm{mg} / \mathrm{Kg} \text { of soil }\end{array}$ & MI, CA & $\mathrm{MC}$ & CT, GT & (Kovalchuk et al., 1998) \\
\hline $\begin{array}{l}\text { Industrial WW } \\
\text { contaminated soil }\end{array}$ & $\begin{array}{l}\text { Metals }(\mathrm{Hg}, \mathrm{Cd}, \mathrm{As}, \mathrm{Cu}, \mathrm{Ni}, \mathrm{Cr}, \mathrm{Pb}, \mathrm{Zn}) \text {, PAHs }(\mathrm{n}=7), \mathrm{PCBs}(\mathrm{n}=9) \text { and } \\
\text { other organic compound used as solvents }(\mathrm{n}=14)\end{array}$ & MI, MN & $\mathrm{F}_{1}$ & GT, MT & (Cotelle et al., 1999) \\
\hline $\begin{array}{l}\text { Textile \& paper mills } \\
\text { WW }\end{array}$ & Wastewater and sludge (50\%): Composition not reported & $\mathrm{CA}, \mathrm{MN}$ & MC & GT, MT & (Grover and Kaur, 1999) \\
\hline Mixture & Municipal sludge sample before and after bioremideation & $\begin{array}{l}\text { MI, CA, MN, } \\
\text { NA }\end{array}$ & MC & $\begin{array}{l}\text { GT, GT, } \\
\text { MT }\end{array}$ & $\begin{array}{l}\text { (Cabrera and Rodriguez, } \\
\text { 1999) }\end{array}$ \\
\hline Farmland soil & Irrigated with mixture of industrial watewater & $\mathrm{MN}, \mathrm{CA}$ & MC & GT, CT & $\begin{array}{l}\text { (Cabrera and Rodriguez, } \\
\text { 1999) }\end{array}$ \\
\hline \multirow[t]{2}{*}{$\begin{array}{l}\text { Pesticides } \\
\text { contaminated soil }\end{array}$} & Pesticide (Metolachlor & $\mathrm{CA}$ & $\mathrm{MC}$ & GT & (Kong and Ma, 1999) \\
\hline & $\begin{array}{l}\text { Atrazine, Extrazine and 2,4-D) contaminated soil sampling and soil } \\
\text { practing organic forming }\end{array}$ & & & & \\
\hline Mineral water & $\begin{array}{l}\text { commercial non-carbonated mineral waters stored at different } \\
\text { condition, PET bottled and glass bottle: } \mathrm{pH}-7.33 \text {, TDS-330, Free } \mathrm{CO} 2- \\
43, \mathrm{HCO}-325, \mathrm{Ca}^{2+}-72, \mathrm{Mg}^{2+}-24, \mathrm{Ba}^{2+}-0.006, \mathrm{Na}^{+}-16, \mathrm{Sr}^{2+}-0.38, \mathrm{SiO} 2- \\
7.0, \mathrm{Cl}-16.0, \mathrm{SO}_{4}^{2-} 25 \text { and } \mathrm{NO}^{2}-0.9 \mathrm{mg} / \mathrm{L}\end{array}$ & CA, MI, RL & MC & GT, CT & (Evandri et al., 2000) \\
\hline Phosphoric gypsum & $\begin{array}{l}\text { Composition: } \mathrm{Ca}^{2+}(960), \mathrm{SO}^{2-}(3599), \mathrm{P}^{5+}(3200-3800), \mathrm{F}^{-}(2500-3500), \\
\mathrm{Si}^{4+}(800), \mathrm{Na}^{+}(1313), \mathrm{Fe}^{3+}(55), \mathrm{Mg}^{2+}(114), \mathrm{NH}^{4+}(23), \mathrm{Cl}^{-}(40), \mathrm{K}^{+}(53) \\
\text { and SS-9 } \mathrm{mg} / \mathrm{dm}^{3}\end{array}$ & MI, CA & $\mathrm{MC}$ & CT, GT & (Pavlica et al., 2000) \\
\hline \multicolumn{6}{|l|}{ depot WW } \\
\hline $\begin{array}{l}\text { Disinfected drinking } \\
\text { water }\end{array}$ & $\begin{array}{l}\text { Chlorine dioxide, ozone, peracetic acid and UV radiation. Before } \\
\text { disinfection raw water properties: winter (COD-29.7, SS- } 4.3 \text {, nitrate- } \\
10.1 \text {, nitrite- }<0.2 \text {, ammonia- }<1, \mathrm{mg} / \mathrm{L}, \mathrm{pH}-7.2, \mathrm{~T}-12.9^{\circ} \mathrm{C} \text { and redox } \\
\text { potential- } 168 \mathrm{mV} \text { and Summar (COD- } 21.8, \mathrm{SS}-4.8 \text {, nitrate- } 10.9 \text {, nitrite- } \\
<0.2 \text {, ammonia- }<1 \text {, mg/L, pH-7.1, T- } 21^{0} \mathrm{C} \text { and redox potential-79 } \mathrm{mV} \text { ) }\end{array}$ & $\mathrm{MN}$ & $\mathrm{MC}$ & MT & (Monarca et al., 2002) \\
\hline $\begin{array}{l}\text { Silk dying industry } \\
\text { WW }\end{array}$ & Composition not reported & MI, NA & $\mathrm{MC}$ & GT, CT & (Sudhakar et al., 2001) \\
\hline $\begin{array}{l}\text { Fertilizer and } \\
\text { petrochemical WW }\end{array}$ & $\begin{array}{l}\mathrm{pH}-7.7, \mathrm{EC}-461 \mathrm{uS} / \mathrm{cm}, \mathrm{NH}-<0.05, \mathrm{NO} 2-0.03, \mathrm{NO}-1.58, \mathrm{Cl}-26.8, \mathrm{SO} 4- \\
40.8, \mathrm{PO}-0.16, \mathrm{CN}-<0.01 \text {, mineral oil-0.045 mg/L and Fe-0.03, Mn, Pb, } \\
\mathrm{Cd}, \mathrm{Zn}, \mathrm{Cr}, \mathrm{Hg}, \mathrm{As}, \mathrm{Cu},<0.05 \mathrm{mg} / \mathrm{L} \text { and dichlorethane-<0.1, Toulene-2.8, } \\
\text { Xylene-2.8, PCB-<0.001 and PAH-0.02 ug/L }\end{array}$ & $\mathrm{CA}$ & $\mathrm{MC}$ & GT & (Vujosevic et al., 2001) \\
\hline $\begin{array}{l}\text { Carbon electrode } \\
\text { production factory }\end{array}$ & $\begin{array}{l}\text { Soil samples were collected from a contaminated area near a factory, } \\
\text { composition: Benzo(a)anthracene, Benzo(b)uoranthene, } \\
\text { Benzo(k)uoranthene, Benzo(a)pyrene, Dibenzo(a,h)anthracene are } \\
\text { equal to } 61.2 \mathrm{mg} / \mathrm{Kg} \text { and other PAH (Phenantrene, Anthracene, } \\
\text { Fluoranthene, Pyrene and Chrysene) and heavy metals (Cd, Pb, Cu, } \mathrm{Zn} \text {, } \\
\mathrm{Hg} \text { ) }\end{array}$ & $\mathrm{MN}$ & $\mathrm{MC}$ & MT & (Monarca et al., 2002) \\
\hline
\end{tabular}


Table 2: Continue...

\begin{tabular}{|c|c|c|c|c|c|}
\hline $\begin{array}{l}\text { Radium production } \\
\text { industry }\end{array}$ & $\begin{array}{l}\text { Water collected from natural reservoirs located near the radium } \\
\text { production industry storage cell, composition: } \mathrm{U}^{238}, \mathrm{Th}^{232}, \mathrm{RA}^{226}, \mathrm{Po}^{210} \text {, } \\
\mathrm{Pb}^{210}, \mathrm{Ca}, \mathrm{Mg}, \mathrm{K}, \mathrm{Na}, \mathrm{Cu}, \mathrm{Pb}, \mathrm{Cd}, \mathrm{Zn}, \mathrm{Mn}, \mathrm{Ni}\end{array}$ & MI, CA & MC & GT, CT & (Evseeva et al., 2003) \\
\hline $\begin{array}{l}\text { Mixed industrail } \\
\text { WW }\end{array}$ & 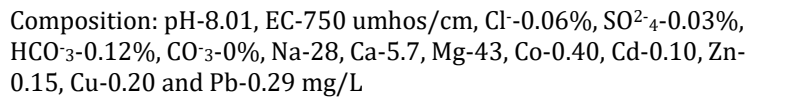 & CA, MN, MI & MC & $\begin{array}{l}\text { GT, CT, } \\
\text { MT }\end{array}$ & (El-Shahaby et al., 2003) \\
\hline $\begin{array}{l}\text { Mixed industrail } \\
\text { WW }\end{array}$ & $\begin{array}{l}\text { Composition: } \mathrm{pH}-8.18, \mathrm{EC}-650 \text { umhos/cm, } \mathrm{Cl}^{-}-0.06 \%, \mathrm{SO}^{2-}{ }^{-}-0.04 \% \text {, } \\
\mathrm{HCO}^{-}{ }_{3}-0.12 \%, \mathrm{CO}_{3}^{-}-0 \% \text {, Na-33, Ca-1.2, Mg-53, Co-0.20, Cd-0.10, Zn- } \\
0.22, \mathrm{Cu}-0.40 \text { and Pb-0.43 mg/L }\end{array}$ & CA, MN, MI & MC & $\begin{array}{l}\text { GT, CT, } \\
\text { MT }\end{array}$ & (El-Shahaby et al., 2003) \\
\hline $\begin{array}{l}\text { Mixed industrail } \\
\text { WW }\end{array}$ & $\begin{array}{l}\text { Composition: } \mathrm{pH}-8.20 \text {, EC-800 umhos/cm, } \mathrm{Cl}^{-}-0.08 \%, \mathrm{SO}^{2-}{ }^{-}-0.04 \% \\
\mathrm{HCO}^{-}{ }_{3}-0.23 \%, \mathrm{CO}^{-} 3-0 \% \text {, Na-35, Ca-1.0, Mg-50, Co-0.40, Cd-0.15, Zn-0.1, } \\
\mathrm{Cu}-0.40 \text { and Pb-0.43 mg/L }\end{array}$ & CA, MN, MI & MC & $\begin{array}{l}\text { GT, CT, } \\
\text { MT }\end{array}$ & (El-Shahaby et al., 2003) \\
\hline $\begin{array}{l}\text { Mixed industrail } \\
\text { WW }\end{array}$ & 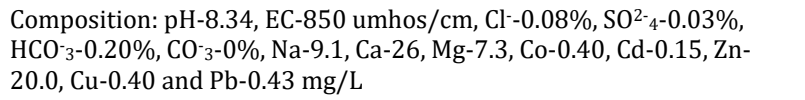 & CA, MN, MI & MC & $\begin{array}{l}\text { GT, CT, } \\
\text { MT }\end{array}$ & (El-Shahaby et al., 2003) \\
\hline Sewage water & $\mathrm{pH}-7.40, \mathrm{~T}-28.92{ }^{\circ} \mathrm{C}, \mathrm{SS}-532.40, \mathrm{DO}-0.73, \mathrm{BOD}-175, \mathrm{COD}-1758 \mathrm{mg} / \mathrm{L}$ & $\begin{array}{l}\text { MI, CA, NA, } \\
\text { MN, RL }\end{array}$ & MC & $\begin{array}{l}\text { CT, MT, } \\
\text { GT }\end{array}$ & $($ Amin, 2002) \\
\hline $\begin{array}{l}\text { Disinfacted drinking } \\
\text { water }\end{array}$ & Disinfacted by $\mathrm{NaClO}, \mathrm{ClO}_{2}$ and peracetic acid & CA & MC & GT & (Monarca et al., 2003) \\
\hline Pulp and paper WW & $\begin{array}{l}\text { Composition: } \mathrm{pH}-9.35, \mathrm{SS}-362.85, \mathrm{COD}-432, \mathrm{EC}-1525 \mathrm{mmho} / \mathrm{cm}, \mathrm{Na}- \\
\text { 227.2, K-24, } \mathrm{Ca}-72.77, \mathrm{Mg}-2.01, \mathrm{Fe}-0.12, \mathrm{Mn}-<0.2, \mathrm{Cr}-<0.4, \mathrm{Ni}-<0.2, \mathrm{Cu}- \\
<0.1, \mathrm{Zn}-0.02, \mathrm{~Pb}-<0.5 \text { and P04-0.66 mg/L }\end{array}$ & RL, MI, CA & MC & CT, GT & (Tipirdamaz et al., 2003) \\
\hline Mixture & $\begin{array}{l}\text { Pulp and paper mill, pharmaceutical, enzyme production plant, oil } \\
\text { refinery, polyester production plant and steel factory. }\end{array}$ & RL & MC & CT & (Pessala et al., 2004) \\
\hline $\begin{array}{l}\text { Metal industry } \\
\text { waste }\end{array}$ & $\begin{array}{l}\text { Composition: } \mathrm{pH}-5.1, \mathrm{COD}-21.7, \mathrm{EC}-2.3 \mathrm{M} \text { mho/cm., Sali-11.9, TN-4.4, } \\
\text { TOC-384.1, TDS-309.0, Cl-600.0, N-1120.0, Na-414.0, K-11.7 mg/L, } \\
\text { heavy metals (Cd-0.01, Cr-282.2, Cu-1.0, Fe-21.0, Mn-1.0, Ni-9.8, Pb- } \\
1.1 \text { and Zn-2.1 ppm) }\end{array}$ & MI, CA, MN & MC & $\begin{array}{l}\text { GT, CT, } \\
\text { MT }\end{array}$ & (Chandra et al., 2005) \\
\hline Dye industry & $\begin{array}{l}\text { Composition: } \mathrm{pH}-6.7, \mathrm{COD}-24.7, \mathrm{EC}-1.3 \mathrm{M} \text { mho/cm, Sali-6.7, TN-90.7, } \\
\text { TOC-138.4, TDS-372.0, Cl-64.2, Nitrate-870.0, Na-53.3, K-3.6 mg/L, } \\
\text { heavy metals (Cd-<0.001, Cr-11.2, Cu-1.6, Fe-4.2, Mn-0.02, Ni-3.6, Pb- } \\
0.5 \text { and Zn-1.2 ppm) }\end{array}$ & MI, CA, MN & MC & $\begin{array}{l}\text { GT, CT, } \\
\text { MT }\end{array}$ & (Chandra et al., 2005) \\
\hline $\begin{array}{l}\text { Disinfected surface } \\
\text { water }\end{array}$ & $\begin{array}{l}\text { Wastewater samples were collected before and after disinfection, in } \\
\text { winter and in summer (peracetic acid and hypochlorite disinfecting } \\
\text { agent) }\end{array}$ & CA, MN, RL & M. C & $\begin{array}{l}\text { GT, CT, } \\
\text { MT }\end{array}$ & (Crebelli et al., 2005) \\
\hline Sewage WW & $\begin{array}{l}\text { Sampling form municipal wastewater treatment plant: composition: } \\
\text { BOD-199-482, COD-458-815, TSS-70-303, TN-29.8-50.3, TP-5.3-11.7 } \\
\text { mg/L }\end{array}$ & MI, CA & $\mathrm{MC}$ & GT, CT & (Grisolia et al., 2005) \\
\hline $\begin{array}{l}\text { Contamianted river } \\
\text { water }\end{array}$ & $\begin{array}{l}\text { heavy metal and cyanide contaminated river waters in a mining region } \\
\text { of Southwest Bulgaria }\end{array}$ & MI, CA & MC & GT, CT & (Ivanova et al., 2005) \\
\hline $\begin{array}{l}\text { Contamianted river } \\
\text { water }\end{array}$ & $\begin{array}{l}\text { Sampling from river contaminated with industrial wastewater } \\
\text { (mixture) }\end{array}$ & MI, CA, MN & MC & $\begin{array}{l}\text { GT, CT, } \\
\text { MT }\end{array}$ & (Srivastava et al., 2005) \\
\hline Contaminated water & Heavy metal $(\mathrm{Cu}, \mathrm{As}, \mathrm{Cd}, \mathrm{Pb})$ and cyanide contaminated waters & CA, NA, MI & MC & CT, GT & (Staykova et al., 2005) \\
\hline Complex mixture & $\begin{array}{l}\text { Industrial effluents: composition heavy metals (Cd-878, Cu-564, Cr-94, } \\
\mathrm{Pb}-324, \mathrm{Zn}-242, \mathrm{ppm} \text { and } \mathrm{Hg}-242 \mathrm{ppb} \text { ) and pesticides }\end{array}$ & $\mathrm{CA}$ & MC & GT & $\begin{array}{l}\text { (Fatima and Ahmad, } \\
\text { 2006b) }\end{array}$ \\
\hline $\begin{array}{l}\text { Chemical fertilizer } \\
\text { WW }\end{array}$ & Composition: Fe-1.1, Pb-1.2, Cr-0.3 and $\mathrm{Cu}-0.12 \mathrm{mg} / \mathrm{L}$ & $\begin{array}{l}\text { NA, CA, MI, } \\
\text { MN }\end{array}$ & MC & $\begin{array}{l}\text { GT, MT, } \\
\text { CT }\end{array}$ & $\begin{array}{l}\text { (Abdel Migid et al., } \\
\text { 2007) }\end{array}$ \\
\hline Complex mixture & Industrial effluent & $\mathrm{CA}, \mathrm{MN}$ & MC & GT, MT & $\begin{array}{l}\text { (Carita and Marin- } \\
\text { Morales, 2008) }\end{array}$ \\
\hline River water & $\begin{array}{l}\text { The river receives municipal wastewater as well as industrial and } \\
\text { agricultural wastewater }\end{array}$ & $\begin{array}{l}\text { CA, NA, MI, } \\
\text { MN, RL }\end{array}$ & MC & $\begin{array}{l}\text { CT, MT, } \\
\text { GT }\end{array}$ & (Egito et al., 2007) \\
\hline $\begin{array}{l}\text { Stream } \\
\text { contaminated with } \\
\text { tannery WW }\end{array}$ & $\begin{array}{l}\text { Composition: Summar (COD-8, BOD-8, pH-6.8, TSS-6, TN-2, O \& G-3, } \\
\text { Cr-1.5-13, T-20 }{ }^{\circ} \mathrm{C}, \mathrm{Ph}-0.02 \text {, DO-6.0 mg/L) and Winter: COD-11, BOD-4, } \\
\text { pH-7.7, TSS-17, TN-1.0, } 0 \text { \& G-9.0, Cr-ND, T-13 }{ }^{\circ} \mathrm{C}, \mathrm{Ph}-\mathrm{ND}, \mathrm{DO}-6.1 \mathrm{mg} / \mathrm{L}\end{array}$ & MI, MN, RL & $\mathrm{MC}$ & GT \& TC & (Junior et al., 2007) \\
\hline $\begin{array}{l}\text { Insecticide and shoe } \\
\text { polish factory WW }\end{array}$ & $\begin{array}{l}\text { Composition: pH-6.3, TDS-520, SO4-71.7-, Cl-90, COD-512, TSS-14700, } \\
\text { Na-275, K-42.5, Ca-1062, Mg-48.6, Co-18.85, Cu-1191.11, Cd-43.75, } \\
\text { Pd-2.30 mg/L }\end{array}$ & CA, MI & MC & CT & $\begin{array}{l}\text { (Abu and Ezeugwu, } \\
\text { 2008) }\end{array}$ \\
\hline
\end{tabular}


Table 2: Continue...

\begin{tabular}{|c|c|c|c|c|c|}
\hline $\begin{array}{l}\text { Aluminum industry } \\
\text { waste }\end{array}$ & $\begin{array}{l}\text { Spent potliners waste; composition: } \mathrm{pH}-9.5, \mathrm{EC}-9.3 \mathrm{dS} / \mathrm{m}, \mathrm{Al}-0.7, \mathrm{CN}^{-}- \\
23.4, \mathrm{~F}-47.8, \mathrm{Cd}-0.18 \mathrm{mg} / \mathrm{L}, \mathrm{Cu}-0.34 \mathrm{mg} / \mathrm{L}, \mathrm{Fe}-75 \mathrm{mg} / \mathrm{L} . \mathrm{Pb}-0.23 \mathrm{mg} / \mathrm{L} \text {, } \\
\text { Mn-0.18 mg/L, Na-657 mg / } \mathrm{L} \text { and } \mathrm{Zn}-0.34 \mathrm{mg} / \mathrm{L}\end{array}$ & MI, CA & MC & CT, GT & (Andrade et al., 2008) \\
\hline Textile WW & $\begin{array}{l}\text { Wastewater contaning dyes (CI Disperse Blue 373, CI Disperse Violet } \\
\text { 93, CI Disperse Orange 37) and aromatic amines }\end{array}$ & MI, CA, MN & MC & $\begin{array}{l}\text { CT, GT, } \\
\text { MT }\end{array}$ & $\begin{array}{l}\text { (Carita and Marin- } \\
\text { Morales, 2008) }\end{array}$ \\
\hline Lemon juice factory & $\begin{array}{l}\text { Composition: } \mathrm{pH}(4.46), \mathrm{EC}(1260 \mathrm{uS} / \mathrm{cm}), \mathrm{DO}(0 \mathrm{mg} / \mathrm{L}), \mathrm{T}\left(22.4^{0} \mathrm{C}\right), \mathrm{SS} \\
(0.70 \mathrm{~g} / \mathrm{L}), \mathrm{COD}(42.23 \mathrm{mg} / \mathrm{L})\end{array}$ & CA, MI, MN & MC & $\begin{array}{l}\text { MT, CT, } \\
\text { GT }\end{array}$ & (Gana et al., 2008) \\
\hline Mining industry & $\begin{array}{l}\text { Composition: pH(6.12), EC(2967 uS/cm), DO }(5.25 \mathrm{mg} / \mathrm{L}), \mathrm{T}\left(23^{\circ} \mathrm{C}\right), \mathrm{SS} \\
(1.80 \mathrm{~g} / \mathrm{L}), \mathrm{COD}(23.57 \mathrm{mg} / \mathrm{L})\end{array}$ & CA, MI, MN & MC & $\begin{array}{l}\text { MT, CT, } \\
\text { GT }\end{array}$ & (Gana et al., 2008) \\
\hline Sugar factory & $\begin{array}{l}\text { Composition: pH( } 4.92), \mathrm{EC}(627 \mathrm{uS} / \mathrm{cm}), \mathrm{DO}(0 \mathrm{mg} / \mathrm{L}), \mathrm{T}\left(42^{\circ} \mathrm{C}\right), \mathrm{SS} \\
(0.40 \mathrm{~g} / \mathrm{L}), \mathrm{COD}(5566 \mathrm{mg} / \mathrm{L})\end{array}$ & CA, MI, MN & MC & $\begin{array}{l}\text { MT, CT, } \\
\text { GT }\end{array}$ & (Gana et al., 2008) \\
\hline Paper factory & $\begin{array}{l}\text { Composition: } \mathrm{pH}(6.12), \mathrm{EC}(4483 \mathrm{uS} / \mathrm{cm}), \mathrm{DO}(0 \mathrm{mg} / \mathrm{L}), \mathrm{T}\left(29^{\circ} \mathrm{C}\right), \mathrm{SS} \\
(1.80 \mathrm{~g} / \mathrm{L}), \mathrm{COD}(6.27 \mathrm{mg} / \mathrm{L})\end{array}$ & CA, MI, MN & MC & $\begin{array}{l}\text { MT, CT, } \\
\text { GT }\end{array}$ & (Gana et al., 2008) \\
\hline River water & $\begin{array}{l}\text { Contaminated with refinery WW, composition: } \mathrm{HC}\left(\mathrm{C}_{12}-\mathrm{C}_{18}\right) \text { and PAHs } \\
\text { (naphthalene, acenaphthylene, acenaphthlene, fluorine, phenanthrene, } \\
\text { pyrene, benzoanthracence and chrysene) }\end{array}$ & $\mathrm{MN}, \mathrm{CA}$ & $\begin{array}{l}\text { MC \& } \\
\mathrm{F}_{1}\end{array}$ & GT, MT & $\begin{array}{l}\text { (Leme and Marin- } \\
\text { Morales, 2008) }\end{array}$ \\
\hline River water & $\begin{array}{l}\text { Oil refinery contamination, composition: } \mathrm{HC}(\mathrm{C} 12-\mathrm{C} 18) \text { and PAHs } \\
\text { (naphthalene, acenaphthylene, acenaphthlene, fluorine, phenanthrene, } \\
\text { pyrene, benzoanthracence and chrysene) }\end{array}$ & MI, CA, MN & $\begin{array}{l}\mathrm{MC} \\
\& \mathrm{~F}_{1}\end{array}$ & $\begin{array}{l}\text { GT, CT, } \\
\text { MT }\end{array}$ & (Leme et al., 2008) \\
\hline Contaminated soil & Composition: $\mathrm{Cr}, \mathrm{Co}, \mathrm{Cu}, \mathrm{Mn}, \mathrm{Hg}, \mathrm{Ni}$ and $\mathrm{Zn}$ & CA & MC & GT & (Katnoria et al., 2008) \\
\hline Mixture & Industrial $\mathrm{WW}$, composition: $\mathrm{Cu}, \mathrm{As}, \mathrm{Cd}, \mathrm{Pb}$ and cyanides & MI, CA, MN & MC & $\begin{array}{l}\text { CT. MT, } \\
\text { GT }\end{array}$ & (Ivanova et al., 2008) \\
\hline Hospital waste & Composition is not reported & $\mathrm{CA}, \mathrm{MN}$ & MC & GT, MT & (Bagatini et al., 2009) \\
\hline River water & $\begin{array}{l}\text { Contaminated with Petroleum refinery WW, composition: Ph-7.4, } \\
\text { BOD-252, COD-848,NH3-19.32, DO-0.0, NO3-8.2, NO2-0.32, SS-1.80, } \\
\text { metal such as Ba-0.27-Cd-<0.003, Cr-<0.005, Co-<0.005, Cu-0.01, Fe- } \\
0.46, \mathrm{~Pb}-<0.02 \text {, Ni-0.02 and Zn-0.18 mg/L and PAH (563-847 ug/kg) }\end{array}$ & MI, CA, MN & MC & $\begin{array}{l}\text { CT, GT, } \\
\text { MT }\end{array}$ & $\begin{array}{l}\text { (Hoshina and Marin- } \\
\text { Morales, 2009) }\end{array}$ \\
\hline silver nanoparticles & $\begin{array}{l}\text { The silver nanoparticles were suspended directly in deionized water } \\
\text { at different concentrations }(25,50,75 \text { and } 100 \mathrm{ppm}) \text {. }\end{array}$ & MI, CA & MC & GT, CT & (Kumari et al., 2009) \\
\hline Gasoline station WW & $\begin{array}{l}\text { Composition: pH-6, COD-404, BOD-180, } 0 \text { \& G-30, SS-34, VSS-17, Fixed } \\
\text { SS-17, benzene-41.8 ug/L, Toluene-291.5 ug/L, xylene-671.4 ug/L, } \\
\mathrm{Ph}-0.68, \mathrm{Cu}-0.027 \text {, NI-0.105 and Zn-0.005 mg/L }\end{array}$ & $\begin{array}{l}\text { NA, CA, MI, } \\
\text { MN }\end{array}$ & MC & $\begin{array}{l}\text { GT, CT, } \\
\text { MT }\end{array}$ & $\begin{array}{l}\text { (Oliveira-Martins and } \\
\text { Grisolia, 2009) }\end{array}$ \\
\hline Sewage water & $\begin{array}{l}\text { Sample was collected before their discharge into the coastal waters } \\
\text { and mile away from discharge point }\end{array}$ & CA, RL & MC & GT & $\begin{array}{l}\text { (Ukaegbu and Odeigah, } \\
\text { 2009) }\end{array}$ \\
\hline Oil refinery WW & $\begin{array}{l}\text { Sampling before and after landforming: } \mathrm{pH}-5.0, \mathrm{OM}-115.0 \mathrm{~g} / \mathrm{dm}^{3}, \mathrm{~K}- \\
\text { 1.8, Ca-75, Mg-27.0, Al-1.0, Na-195.0, Fe-112.0, Mn-13.8, Cu-3.2, Zn- } \\
84.8 \mathrm{mmol} / \mathrm{dm}^{3} \text {, Ba-493.4, Cd-<0.01, Cr-400.2, Ni-104.5, Pb-18.8 mg/L }\end{array}$ & $\mathrm{CA}, \mathrm{MN}$ & MC & CT, MT & (Souza et al., 2009) \\
\hline Industrial WW & $\begin{array}{l}\text { Incoming and outgoing in biological and chemical wastewater } \\
\text { treatment plant in industrial zone }\end{array}$ & MI, CA & MC & GT, CT & (Sik et al., 2009) \\
\hline River water & River water contaminated with industrial wastewater & MI, RL, MN & MC & $\begin{array}{l}\text { CT, GT, } \\
\text { MT }\end{array}$ & (Barberio et al., 2009) \\
\hline Textile effluent & $\begin{array}{l}\text { Composition: pH-8.2, EC-3.48 uS/m, COD-6760, BOD-670, TDS-1040, } \\
\text { TS-9810 and TH-375 mg/L }\end{array}$ & MI, CA, MN & MC & $\begin{array}{l}\text { GT, MT, } \\
\text { CT }\end{array}$ & (Jadhav et al., 2010) \\
\hline Oil refinery WW & $\begin{array}{l}\text { Composition: Toluene-75, Ethylbenzene- } 126 \text {, Xylene- } 85 \text {, pyrene- } 9 \text {, } \\
\text { benzoantracence- } 33 \text { and benzopyrene- } 61 \mathrm{ppb}\end{array}$ & MI, MN & $\mathrm{F}_{1}$ & GT, MT & (Rodrigues et al., 2010) \\
\hline Refinary WW & Composition is not reported & CA, MN & & CT, GT & $\begin{array}{l}\text { (Gupta and Ahmad, } \\
\text { 2011) }\end{array}$ \\
\hline River water & $\begin{array}{l}\text { Contaminated with municipal wastewater, sugar factory, lead smelter, } \\
\text { runoff fromsoil contaminated by agricultural practices, fertilizer plant, } \\
\text { pharmaceutical plant and food industries: Composition: pH-7-8, EC- } \\
431-1642 \text { us/cm, COD-2.15-112.70, BOD-165-16.9, SS-2.0-45.6, } \\
\text { nitrate-1.09-4.67, nitrite-0.02-0.35, Al-0.07-1.64 mg/L }\end{array}$ & RL, MI, CA & MC & GT, CT & (Radic et al., 2010) \\
\hline $\begin{array}{l}\text { Textile and paint } \\
\text { WW }\end{array}$ & Effluents were collected from discharge points of the industries & MI, CA & MC & GT, CT & (Samuel et al., 2010) \\
\hline Paper mill & $\begin{array}{l}\text { bleach plant effluents: sampling after and before biodegradability } \\
\text { from a bench-scale horizontal anaerobic immobilized bioreactor }\end{array}$ & MI, CA, MN & MC & $\begin{array}{l}\text { GT. CT, } \\
\text { MT }\end{array}$ & (Chaparro et al., 2010) \\
\hline Phamarceutical WW & $\begin{array}{l}\text { Composition: pH-2.9, TDS-42000, Cu-20.0, Zn-17.3, Pb-2.2, Cl-0.02, } \\
\text { SO4-696.2 mg/L }\end{array}$ & RL, MI & MC & CT & (Abu and Mba, 2011) \\
\hline River water & $\begin{array}{l}\text { Contaminated with untreated industrial wastewater, composition: } \\
\text { Summar-pH-7.29, EC-29, D0-7.09, T-24.1 }{ }^{\circ} \mathrm{C} \text {, metal (Cu-ND, Cr-ND, Ni- } \\
0.031, \mathrm{~Pb}-0.136, \mathrm{Cd}-0.009, \mathrm{Zn}-0.078 \text { and Fe-0.001 mg/L) }\end{array}$ & CA, MI, MN & MC & $\begin{array}{l}\text { GT, CT, } \\
\text { MT }\end{array}$ & (Bianchi et al., 2011) \\
\hline
\end{tabular}


Table 2: Continue...

River water

River water

River water

Coal mining waste

Contaminated soil

Mixture

Sewage and oil refinery

Mixed WW

Mixture

Mixture

Mixture

Contaminated soil

Textile WW

Industrial and river water

Mixture

River water
Autumn: pH-5.53, EC-9.0 uS/cm2, DO-7.15, T-19.6 ${ }^{\circ} \mathrm{C}$, metal (Cu-ND, Cr-ND, Ni-0.072, Pb-0.192, Cd-0.009, Zn-0.096 and Fe-1.01 mg/L)

Winter: pH-6.85, EC-13uS/cm2, DO-8.65, T-20.9 ${ }^{\circ} \mathrm{C}$, metal (Cu-ND, CrND, Ni-ND, Pb-0.304, Cd-ND,

Fe-0.002 and Ca-1.342 mg/L)

Spring: pH-6.55, EC-12 uS/cm2, D0-7.22, T-20.7 ${ }^{\circ} \mathrm{C}$, metal $(\mathrm{Cu}-0.04, \mathrm{Cr}-$ 0.03, Ni-ND, Pb-0.14, Cd-ND, Zn-0.15, Fe-1.34, Mn-0.10, Na-1.72 $\mathrm{mg} / \mathrm{L}$ )

Composition: Inorganic ions, Metals ( $\mathrm{Ca}, \mathrm{Mg}, \mathrm{Na}, \mathrm{K}, \mathrm{Fe}, \mathrm{Mn}, \mathrm{Ba}, \mathrm{Sr}, \mathrm{Zn}$, $\mathrm{Pb}, \mathrm{Al}, \mathrm{Cr}, \mathrm{Cu}, \mathrm{Ni}, \mathrm{Co}, \mathrm{Cd}, \mathrm{Hg})$ and radionuclides $\left(\mathrm{Ra}^{226}\right.$ and $\left.\mathrm{Ra}^{228}\right)$

soil Contaminated with zinc coating industry wastewater: $\mathrm{pH}-3.46$, nitrate-1.69, P04-3.05, K-0.13, Cu-32.86, Co-6.86, Cr-0.0, Hg-0.037, Mn-1.55, Ni-9.66, Zn-6.53 mg/L

Benzene, toluene, ethylbenzene and xylene (benzene $1.25 \mathrm{ug} / \mathrm{L}$, toluene $42.5 \mathrm{ug} / \mathrm{L}$, ethylbenzene $50 \mathrm{ug} / \mathrm{L}$ and xylenes $75 \mathrm{ug} / \mathrm{L}$ )

Composition: T-25-26 ${ }^{\circ} \mathrm{C}$, EC-119-113, pH-6.8-7.3, DO-3.2-3.3, BOD-23, COD-16-22, NH3-1.15-1.54, ON-1.38-1.85, P04-0.21-0.29, TS-81, srfactatnt-0.023, Cu-0.009, Cl--7.0, Tot. Cr-0.032-0.041, Cr(III)-0.032, $\mathrm{Al}-1.86, \mathrm{Fe}-2.19, \mathrm{Zn}-0.025-0.034 \mathrm{mg} / \mathrm{L}$

Sewage, Leather, agriculture, paper recycling industry, composition: T25-34 ${ }^{\circ} \mathrm{C}, \mathrm{EC}-119-113$, pH-6.9-7.1, DO-0.3-2, BOD-3 COD-20-22, NH31.28-2.33, 0N-1.20-2.71, PO4-0.20-0.26, TS-123-164, srfactatnt-0.14, Cu-0.009, Cl-112.2-18, Tol. Cr-0.01-0.03, Cr(III)-0.03, Al-0.52, Fe-1.47, $\mathrm{Zn}-0.031 \mathrm{mg} / \mathrm{L}$

Sewage and tannery WW, composition: T-17-24 ${ }^{\circ} \mathrm{C}, \mathrm{EC}-156-130, \mathrm{pH}$ 7.0-7.1, DO-0.2-0.3, BOD-3 COD-15-85, NH3-2.19-14.3, ON-2.14-5.0, P04-0.34-0.40, TS-101-637, Phenol-0.035, srfactatnt-0.56, Cu-0.008, Cl-7.4-130.0, Tol. Cr-0.15-0.034, Cr(III)-0.034, Al-4.78, Fe-2.66, Zn$0.102 \mathrm{mg} / \mathrm{L}$

Sewage and matallurgy WW, composition:T-18-23 ${ }^{\circ} \mathrm{C}, \mathrm{EC}-92-145, \mathrm{pH}-$ 6.7-7.1, DO-1.4-2.0, BOD-2-19, COD-2-18, NH3-0.81-1.91, ON-0.300.60 , PO4-0.30-1.17, TS-100-110, phenol-0, srfactatnt-0.12, Cu-0.0060.01, Cl-8.5-11.0, Tol. Cr-0.03, Cr(III)-0.03, Al-1.29, Fe-3.19, Zn-0.023 $\mathrm{mg} / \mathrm{L}$

Sewage, footwear companies and leather finish: T-18-23 ${ }^{\circ} \mathrm{C}, \mathrm{EC}-471-$ 482, pH-6.7-7.0, DO-0.5, BOD-8-17, COD-85-89, NH3-11-12, ON-5.306.9, P04-1.04-1.46, TS-291-300, phenol-0.008, srfactatnt-1.10-2.52, Cu-0.15, Cl-26-39, Tol. Cr-0.058, Cr(III)-0, Al-0, Fe-0, Zn-0.188-0.31 $\mathrm{mg} / \mathrm{L}$

A. cepa grown directly in industrial wastewater irrigated agricultural filed

Wastewater before and after treatment: pH-8.1, COD-9860, BOD-880, TDS-8756, TSS-1089, TS-9845, TH-689, F-12, Cl-1254, SO4-1123, Cu1.3, Fe-1.7, Mg-1.43, Pb-0.63, Ni-0.79, Ca-34.43, K-23.65 and Na-89 $\mathrm{mg} / \mathrm{L}$

Composition: heavy metal $(\mathrm{Cu}>\mathrm{Ni}>\mathrm{Cd})$ and phenolic compounds

Industrial wastewater: BOD-82-378, COD-283-1259, TOC-553-2932 Cd-2.1-672, Cr-3.3-74, Cu-8-376, Fe-8.9-104, Pb-1.3-216 and Hg-0.2$0.4 \mathrm{mg} / \mathrm{L}$

Rainy season site A: Sample collection at point of mixing of metabisulfite and EC-374, H-12.24, Cl-93, DO-6.96, BOD-6, N-0.35, NH3-4.00, pH-6.8, Ca, Mg, K, Na (268, 137, 4.0, $1.1 \mathrm{Meq} / 100 \mathrm{~g}$ soil) and site B: EC-392, H-7.90, Cl-16.45, D0-7.12, BOD-2, N-0.20, pH-6.5, Ca, $\mathrm{Mg}, \mathrm{K}, \mathrm{Na}(139,94,4.6,0.9 \mathrm{Meq} / 100 \mathrm{~g}$ soil $)$

Dry season site A: Sample collection at point of mixing of metabisulfite and EC-44.0, H-35.50, Cl-10.73, DO-5, BOD-2.5, N-0.32, NH3-0.03, pH6.9, $\mathrm{Ca}, \mathrm{Mg}, \mathrm{K}, \mathrm{Na}(100,94,4.6,1.7 \mathrm{Meq} / 100 \mathrm{~g}$ soil) and site B: EC-50, H-10.50, Cl-12.9, DO-6.5, BOD-2.5, N-0.2, NH3-0.01, pH-6.5, Ca, Mg, K, $\mathrm{Na}(139,58,3.7,1.0 \mathrm{Meq} / 100 \mathrm{~g}$ soil $)$

\begin{tabular}{|c|c|c|c|}
\hline CA, MI, MN & MC & $\begin{array}{l}\text { GT, CT, } \\
\text { MT }\end{array}$ & (Bianchi et al., 2011) \\
\hline CA, MI, MN & MC & $\begin{array}{l}\text { GT, CT, } \\
\text { MT }\end{array}$ & (Bianchi et al., 2011) \\
\hline CA, MI, MN & MC & $\begin{array}{l}\text { GT, CT, } \\
\text { MT }\end{array}$ & (Bianchi et al., 2011) \\
\hline MI, CA & MC & GT, CT & (Geras'kin et al., 2011) \\
\hline CA & MC & GT & (Katnoria et al., 2011) \\
\hline $\mathrm{MN}, \mathrm{CA}$ & $\begin{array}{l}\text { MC \& } \\
F_{1}\end{array}$ & GT & (Mazzeo et al., 2011) \\
\hline MN, MI & MC & GT, MT & (Nunes et al., 2011) \\
\hline MN, MI & MC & GT, CT & (Nunes et al., 2011) \\
\hline
\end{tabular}

MN, MI MC GT, CT (Nunes et al., 2011)

MN, MI MC GT, CT $\quad$ (Nunes et al., 2011)

MN, MI MC CT, GT $\quad$ (Nunes et al., 2011)

CA, MN, RL MC GT, MT, (DI and MW, 2011)

MI, CA MC CT, GT $\quad$ (Phugare et al., 2011)

CA MC GT $\quad$ (Siddiqui et al., 2011)

MI, CA, MN MC GT $\quad$ (Tabrez and Ahmad, 2011)

GT, CT, (da Costa Machado

MI, CA, MN MC GT, Matos Carvalho et al., 2011)

MI, CA, MN $\quad$ MC

GT, MT, (da Costa Machado CT Matos Carvalho et al., 2011) 
Table 2: Continue...

\begin{tabular}{|c|c|c|c|c|c|}
\hline River water & $\begin{array}{l}\text { Upstream to metabisulfite discharge point: contaning sodium } \\
\text { metabisulfite (SMB) and the point where sodium metabisulfite is } \\
\text { discharged in river }\end{array}$ & $\begin{array}{l}\text { MI, CA, MN, } \\
\text { RL }\end{array}$ & MC & $\begin{array}{l}\text { GT, MT, } \\
\text { CT }\end{array}$ & $\begin{array}{l}\text { (da Costa Machado } \\
\text { Matos Carvalho et al., } \\
\text { 2011) }\end{array}$ \\
\hline River wastewater & $\begin{array}{l}100 \mathrm{~m} \text { downstream the point of sodium metabisulfite discharge in } \\
\text { river }\end{array}$ & $\begin{array}{l}\text { MI, CA, MN, } \\
\text { RL }\end{array}$ & MC & $\begin{array}{l}\text { GT, MT, } \\
\text { CT }\end{array}$ & $\begin{array}{l}\text { (da Costa Machado } \\
\text { Matos Carvalho et al., } \\
\text { 2011) }\end{array}$ \\
\hline $\begin{array}{l}\text { Municipal } \\
\text { wastewater }\end{array}$ & Sampling form treatment plant after ozonation form reactors & MI, MN & MC & GT, MT & (Misik et al., 2011) \\
\hline $\begin{array}{l}\text { Bottling industry } \\
(10 \%)\end{array}$ & $\begin{array}{l}\text { Composition: T-30² } \text {, pH-10.5, Turb-68FTU, TH-417, TSS-43, TDS-430, } \\
\text { BOD-19.90, DO-2.13, Alkalinity-750, TS-715, Nitrate-50, } \mathrm{SO}_{4}-78, \mathrm{PO}_{4-} \\
0.91, \mathrm{Cl}-248.50, \mathrm{Ca}-306, \mathrm{Cu}-0.02, \mathrm{Mn}-0.1, \mathrm{~Pb}-0.1, \mathrm{Fe}-0.64, \mathrm{Cd}-0.07, \mathrm{Ni}- \\
0.12, \mathrm{Zn}-0.06 \mathrm{mg} / \mathrm{L}\end{array}$ & MI, CA & MC & CT, GT & (Olorunfemi et al., 2011) \\
\hline $\begin{array}{l}\text { Rubber industry } \\
(10 \%)\end{array}$ & $\begin{array}{l}\text { Composition: T- } 31^{0} \mathrm{C} \text {, pH-4.75, Turb-98FTU, TH-320, TSS-40, TDS-720, } \\
\text { BOD-12, DO-4.24, Alkalinity-340, TS-1012, Nitrate-86.50, SO } 4-67.50 \text {, } \\
\left.\mathrm{PO}_{4}-1.15, \mathrm{Cl}-276.90, \mathrm{Ca}-220, \mathrm{Cu}-0.1, \mathrm{Mn}-0.1, \mathrm{~Pb}-0.1, \mathrm{Fe}-0.24\right), \mathrm{Cd}-0.01 \text {, } \\
\mathrm{Ni}-0.1 \text { and } \mathrm{Zn}-0.3 \mathrm{mg} / \mathrm{L}\end{array}$ & MI, CA & MC & CT, GT & (Olorunfemi et al., 2011) \\
\hline $\begin{array}{l}\text { Brewery industry } \\
(10 \%)\end{array}$ & $\begin{array}{l}\text { Composition: T-280 C, pH-5.5, Turb-38FTU, TH-298, TSS-48, TDS-1500, } \\
\text { BOD-15.10, DO-2.72, Alkalinity-230, TS-1970, Nitrate-54, } \mathrm{SO}_{4}-18, \mathrm{PO}_{4}- \\
0.28, \mathrm{Cl}^{-}-335, \mathrm{Ca}-250, \mathrm{Cu}-0.32, \mathrm{Mn}-0.1, \mathrm{~Pb}-0.1, \mathrm{Fe}-0.40, \mathrm{Cd}-0.03, \mathrm{Ni}^{-} \\
0.15, \mathrm{Zn}-2.03 \mathrm{mg} / \mathrm{L}\end{array}$ & MI, CA & M.C & $\mathrm{CT}, \mathrm{GT}$ & (Olorunfemi et al., 2011) \\
\hline Textile dyes mixture & $\begin{array}{l}\text { Nitroaminoazobenzene based dyes: C.I. Disperse Blue 373, C.I. } \\
\text { Disperse Violet } 93 \text { and C.I. Disperse Orange } 37\end{array}$ & NA, CA & MC & CT, GT & $\begin{array}{l}\text { (Ventura-Camargo et al., } \\
\text { 2011) }\end{array}$ \\
\hline Textile WW & $\begin{array}{l}\text { Composition: BOD-152, COD-319, TSS-1160, O \& G-612, pH-6.9, Cr- } \\
1.20, \mathrm{Cu}-0.02, \mathrm{NH}_{4}^{+}-0.50 \mathrm{mg} / \mathrm{L}\end{array}$ & CA, MI, NA & MC & GT, CT & (Oriaku et al., 2011) \\
\hline Contaminated soil & Agricultural soil irrigated with industrial WW and pesticide sprayed & CA & MC & GT & (Chahal et al., 2012) \\
\hline River water & $\begin{array}{l}\text { Contaminated with industrial wastewater: PHAs, acenaphthylene, } \\
\text { acenaphthene, fluorene, phenanthrene, anthracene, fluoranthene, } \\
\text { pyrene,benzo(a)anthracene, chrysene,benzo(b)fluoranthene, ben- } \\
\text { zo(k)fluoranthene, benzo(a)pyrene, indeno(1,2,3-cd)pyrene, } \\
\text { dibenz(a,h)anthracene andbenzo(ghi)perylene. }\end{array}$ & MI, CA & MC & GT, CT & (da Costa et al., 2012) \\
\hline $\begin{array}{l}\text { Disinfacted drinking } \\
\text { water }\end{array}$ & Disinfacted ground and surface water for drinking purpose & CA, MN, RL & MC & GT, MT & (Feretti et al., 2012) \\
\hline Acid mine drainage & $\begin{array}{l}\text { Before and after calcination of coal mining waste: } \mathrm{pH}-2.6, \mathrm{Al}-28.99, \mathrm{Fe}- \\
13.23, \mathrm{Zn}-1.19 \mathrm{Cu}-0.01, \mathrm{~Pb}-<0.0001 \mathrm{mg} / \mathrm{L} \mathrm{SO}_{4}^{-2}-2.17 \mathrm{~g} / \mathrm{L}\end{array}$ & CA & MC & GT & (Geremias et al., 2012) \\
\hline Refinery WW & Composition is not reported & CA, MN, RL & MC & CT, GT & $\begin{array}{l}\text { (Gupta and Ahmad, } \\
\text { 2012) }\end{array}$ \\
\hline $\begin{array}{l}\text { Drainage pumping } \\
\text { stations }\end{array}$ & $\begin{array}{l}\text { Landfill leachates sampling, composition: } \mathrm{Pb}, \mathrm{Ni}, \mathrm{Cd}, \mathrm{Hg} \text {, benzene, } \\
\text { dichloromethane, chloroform, trichlorobenzenes, exachlorobenzene, } \\
\text { hexachlorobutadiene, hexachlorocyclohexane,lindane, } \\
\text { hexachlorocyclohexane, pentachlorobenzene, pentachlorophenol, } \\
\text { PAHs. }\end{array}$ & CA, MN, MI & MC & $\begin{array}{l}\text { GT, MT, } \\
\text { CT }\end{array}$ & $\begin{array}{l}\text { (Kwasniewska et al., } \\
\text { 2012) }\end{array}$ \\
\hline Olive mill WW & $\begin{array}{l}\text { Composition: pH-4.83, EC-11.5 ds/m, COD-65.4, BOD-29.3 g/L, } \\
\text { phenolic contents- } 4.36 \mathrm{ugGA} / \mathrm{mL}\end{array}$ & MI, CA & MC & $\mathrm{CT} \& \mathrm{GT}$ & (Pierantozzi et al., 2012) \\
\hline Soil sample & Contaminated with Cs-137 and Sr-90 & CA & MC & GT & (Kovalchuk et al., 1998) \\
\hline Surface water & $\begin{array}{l}\text { Surface water treated with different disinfecting agent: } \mathrm{ClO}_{2}-1.6 \mathrm{mg} / \mathrm{L} \text {, } \\
\mathrm{NaClO}-1.2 \mathrm{mg} / \mathrm{L} \text {, peracetic acid- } 1 \mathrm{mg} / \mathrm{L}\end{array}$ & MI, CA & MC & GT, CT & (Monarca et al., 2003) \\
\hline Hospital waste & Hospital incinerator bottom ash leachate samples & CA & MC & GT & (Akinbola et al., 2011) \\
\hline Mixture & $\begin{array}{l}\text { Plasticizer, cosmetics, healthcare and preservative agent containing } \\
\text { WW }\end{array}$ & MI, CA, MN & MC & $\begin{array}{l}\text { CT, GT, } \\
\text { MT }\end{array}$ & (Herrero et al., 2012) \\
\hline $\begin{array}{l}\text { Aligarh and Mathura } \\
\text { refinery }\end{array}$ & City wastewater and refinery wastewater & CA & MC & GT & $\begin{array}{l}\text { (Fazili and Ahmad, } \\
\text { 2014) }\end{array}$ \\
\hline Pulp and paper mill & Wastewater bleaching sequence of Kraft cellulose & CA, MN, MI & MC & $\begin{array}{l}\text { CT, GT, } \\
\text { MT }\end{array}$ & (Roa et al., 2012) \\
\hline $\begin{array}{l}\text { Nigerian Agip Oil } \\
\text { Company }\end{array}$ & Wastewater at different concentrations & MI, CA & MC & CT, GT & (Olorunfemi et al., 2015) \\
\hline $\begin{array}{l}\text { Paint industry } \\
\text { effluent }\end{array}$ & Concentrations $(100 \%, 50 \%, 25 \%, 10 \%, 5 \%, 1 \%, 0.50 \%$ and $0.25 \%)$ & CA, MI & MC & CT, GT & (Njoku et al., 2015) \\
\hline Wastewater & Public hospital of Buenos Aires (Argentina) & $\mathrm{CA}$ & MC & GT & (Magdaleno et al., 2014) \\
\hline Wastewater & Buenos Aires (Argentina) & CA & MC & GT & (Paz et al., 2006) \\
\hline Wastewater & hospital effluent in Santa Maria, Rio Grande do Sul State, Brazil & CA, MN & MC & GT, MT & Bagatini et al., 2009 \\
\hline
\end{tabular}


Table 2: Continue...

\begin{tabular}{|c|c|c|c|c|c|}
\hline $\begin{array}{l}\text { Shkodra city } \\
\text { wastewater }\end{array}$ & Sampling from four sites & CA, MI, & MC & CT, GT & $\begin{array}{l}\text { (Mesi and Kopliku, } \\
\text { 2012) }\end{array}$ \\
\hline Wastewater & $\begin{array}{l}\text { industrial wastewaters reaching the Dandugan Oya, Srilanka and the } \\
\text { downstream water }\end{array}$ & CA, MI & MC & CT, GT & $\begin{array}{l}\text { (Kannangara and } \\
\text { Pathiratne, 2015) }\end{array}$ \\
\hline Wastewater & Near the Sava River, Croatia & CA, MI & MC & CT, GT & (Radić et al., 2010) \\
\hline Wastewater & $\begin{array}{l}\text { Un-treated and Upflow Immobilized Biomass Anaerobic Reactor } \\
\text { hospital treated wastewater }\end{array}$ & MI & MC & $\mathrm{CT}$ & (Torres et al., 2014) \\
\hline Wastewater & Industrial and contaminated river water & CA, MI & MC & GT, CT & (Siddiqui et al., 2011) \\
\hline Wastewater & Effluent from tobacco industry & CA, MI & MC & GT, CT & $\begin{array}{l}\text { (Akinsemolu et al., } \\
\text { 2015) }\end{array}$ \\
\hline Wastewater & Distillary waste from a sugarcane factory, Kanpur, India & & & & (Mishra, 1993) \\
\hline Compost & Compost from TPK Sarimukti, Cipatat, West Bandung (solid \& liquid) & $\begin{array}{l}\text { CA, MN, MI, } \\
\text { NA }\end{array}$ & MC & $\begin{array}{l}\text { CT, GT, } \\
\text { MT }\end{array}$ & (Wardini et al., 2015) \\
\hline Wastewater & $\begin{array}{l}\text { Un-treated and treated textile wastewater by advanced oxidation } \\
\text { process }\end{array}$ & RG & MC & CT & (Iqbal et al., 2015) \\
\hline Wastewater & $\begin{array}{l}\text { Un-treated and photo-catalytic treated textile waster Textile } \\
\text { wastewater }\end{array}$ & RG & MC & $\mathrm{CT}$ & (Iqbal and Bhatti, 2014) \\
\hline River water & $\begin{array}{l}\text { Sampling from contaminated river Vallkärrabäcken, Sweden } 34 \text { years } \\
\text { after the closure of the landfill, Sankt Hans }\end{array}$ & RG & MC & CT & (Lindqvist, 2015) \\
\hline Leachate & $\begin{array}{l}\text { Landfill leachate collected from city of Araranguá (Santa Catarina } \\
\text { state, Brazil) }\end{array}$ & RG & MC & CT & (do Canto et al., 2013) \\
\hline Wastewater & $\begin{array}{l}\text { Effluents from six pharmaceutical companies in the Lagos province, } \\
\text { Nigeria }\end{array}$ & RG, MI, CA & MC & CT, GT & (Akintonwa et al., 2009) \\
\hline Wastewater & Industrial effluents contain copper, cadmium and chromium metals & MI, CA, NA & MC & $\begin{array}{l}\text { CT, GT, } \\
\text { MT }\end{array}$ & $\begin{array}{l}\text { (Hemachandra and } \\
\text { Pathiratne, 2015) }\end{array}$ \\
\hline Soil sample & $\begin{array}{l}\text { Soils contaminated by heavy metals from municipality of Triunfo, state } \\
\text { of Rio Grande do Sul, Brazil }\end{array}$ & MI, CA, & MC & CT, GT & $\begin{array}{l}\text { (de Souza Pohren et al., } \\
\text { 2013) }\end{array}$ \\
\hline River water & Sungai Dua River contaminated with sodium and calcium metals & RG, MI, CA & & & (Akinboro et al., 2011) \\
\hline Nanoparticles & $\mathrm{ZnO}$ dispersions at $25,50,75$, and $100 \mu \mathrm{g} / \mathrm{mL}$ concentrations & MI, CA, MN & MC & $\begin{array}{l}\text { CT, GT, } \\
\text { MT }\end{array}$ & (Kumari et al., 2011) \\
\hline Nanoparticles & Cobalt and zinc oxide NPs at 5,10 , and $20 \mu \mathrm{g} / \mathrm{mL}$ concentrations & RG & MC & $\mathrm{CT}$ & (Ghodake et al., 2011) \\
\hline River water & $\begin{array}{l}\text { Jaguari River and the Ribeirão Lavapés, Brazil samples collected in dry } \\
\text { season (August } 2011 \text { and 2012) and rainy season (February } 2012 \text { and } \\
\text { 2013), Mn concentration } 0.13 \mathrm{mg} / \mathrm{L} \text {, greater than permissible limit }\end{array}$ & MI, CA, MN & MC & $\begin{array}{l}\text { CT, GT, } \\
\text { MT }\end{array}$ & (Salles et al., 2016) \\
\hline River water & $\begin{array}{l}\text { Wastewater collected in the years of } 2005 \text { and } 2006 \text { from sites in the } \\
\text { cities of Tremembé and Aparecida (São Paulo state, Brazil) }\end{array}$ & RG, MI & MC & CT & (Barbério et al., 2009) \\
\hline Ecballium elaterium & $\begin{array}{l}\text { Roots were exposed to } 10 \mathrm{~L}, 20,50 \mathrm{ml} / \mathrm{L} \text { and undiluted juice } \\
\text { concentrations }\end{array}$ & $\mathrm{MI}, \mathrm{CA}, \mathrm{MN}$ & MC & $\begin{array}{l}\text { CT, GT, } \\
\text { MT }\end{array}$ & $\begin{array}{l}\text { (Çelik and Aslantürk, } \\
\text { 2009) }\end{array}$ \\
\hline River water & $\mathrm{Cr}(\mathrm{VI})$ contaminated reiver water & MI, RG & MC & CT & $\begin{array}{l}\text { (Espinoza-Quiñones et } \\
\text { al., 2009) }\end{array}$ \\
\hline Radioactive material & $\begin{array}{l}\text { thallium-232 in the presence of different cadmium and potassium } \\
\text { concentrations }\end{array}$ & $\mathrm{CA}, \mathrm{MN}$ & MC & GT, MT & (Evseeva et al., 2005) \\
\hline Wastewater & $\begin{array}{l}\text { Textile industries, rubber industry, treatment plants of industrial } \\
\text { zones }\end{array}$ & MI, CA & MC & CT, GT & (Pathiratne et al., 2015) \\
\hline Leachate & Leachate collected from landfill, Vale do Rio dos Sinos, southern Brazil & RG, MI, CA & MC & CT, GT & (Klauck et al., 2013) \\
\hline Leachate & $\begin{array}{l}\text { Leachate-municipal solid waste landfill, Sinos River Valley, southern } \\
\text { Brazil }\end{array}$ & RG & MC & $\mathrm{PT}$ & (Klauck et al., 2015) \\
\hline Textile wastewater & Bio-treated textilel effluent & RG & MC & $\mathrm{TC} / \mathrm{CT}$ & (Patel et al., 2015) \\
\hline Wastewater & Industry producing veterinary medicines, before and after treatment & RG & MC & $\mathrm{TC}$ & (Maselli et al., 2015) \\
\hline Stream water & $\begin{array}{l}\text { Water samples were collected from three streams located in the urban } \\
\text { area of a municipality in the south of Brazil }\end{array}$ & $\mathrm{MI}, \mathrm{MN}, \mathrm{CA}$ & MC & $\begin{array}{l}\text { CT, GT, } \\
\text { MT }\end{array}$ & (Athanásio et al., 2014) \\
\hline Wastewater & $\begin{array}{l}\text { Hospital laundry wastewaters, Rio Pardo Valley, Rio Grande do Sul, } \\
\text { Brazil }\end{array}$ & CA, MI & MC & CT, GT & (Kern et al., 2015) \\
\hline Sugar industry & $\begin{array}{l}\text { Vermicomposted pressmud (waste by product of the sugar cane } \\
\text { industry) }\end{array}$ & CA, MI & MC & CT, GT & (Bhat et al., 2014) \\
\hline Leachate & Leachate from an old sanitary landfill before and after treatment & CA, MI & MC & CT, GT & (Brkanac et al., 2014) \\
\hline Dye mixture & $\begin{array}{l}\text { Dyes mixture before and after Petunia grandiflora and Gaillardia } \\
\text { grandiflora treatment }\end{array}$ & CA, MI & MC & CT, GT & $\begin{array}{l}\text { (Watharkar and Jadhav, } \\
\text { 2014) }\end{array}$ \\
\hline Contaminated water & $\begin{array}{l}\text { Natural waters contaminated with }{ }^{90} \mathrm{Sr} \text { and heavy metals near } \\
\text { radioactive waste storage, Obninsk, Kaluga region }\end{array}$ & $\mathrm{MN}$ & MC & MT & (Udalova et al., 2014) \\
\hline
\end{tabular}


Table 2: Continue...

\begin{tabular}{|c|c|c|c|c|c|}
\hline River water & Water collected from Quatorze River, Francisco Beltrão, Paraná, Brazil & MI & MC & CT & (Düsman et al., 2014) \\
\hline Dye & Direct Blue- 1 before and after bio-treatment & $\mathrm{CA}$ & MC & GT & (Prasad et al., 2013) \\
\hline Leachate & Treated landfill leachate from the Piškornica (Croatia) sanitary landfill & MI & MC & CT & $\begin{array}{l}\text { (Garaj-Vrhovac et al., } \\
\text { 2013) }\end{array}$ \\
\hline Wastewater & $\begin{array}{l}\text { Liquid waste produced in the process of industrialization of the bitter } \\
\text { cassava, olho-junto variety }\end{array}$ & MI, CA & MC & CT, GT & (Viana et al., 2014) \\
\hline Wastewater & Metal rich acid mine drainage & CA & MC & MT, GT & (Sobral et al., 2013) \\
\hline Wastewater & Industrial wastewaters from Jajmau (Kanpur) & $\mathrm{MI}, \mathrm{CA}$ & MC & GT, CT & $\begin{array}{l}\text { (Masood and Malik, } \\
\text { 2013b) }\end{array}$ \\
\hline Wastewater & Treated and un-treated acid mine drainage & MI, CA, & $\mathrm{MC}$ & GT, CT & (Netto et al., 2013) \\
\hline Contaminated soil & $\begin{array}{l}\text { Soil samples from agricultural fields near industrial area, Jajmau, } \\
\text { Kanpur (India) }\end{array}$ & MI, CA & MC & GT, CT & $\begin{array}{l}\text { (Masood and Malik, } \\
\text { 2013a) }\end{array}$ \\
\hline Wastewater & $\begin{array}{l}\text { Hospital laundry wastewaters (COD - } 3343.8 \mathrm{mg} \mathrm{L}(-1)) \text {, biochemical } \\
\text { oxygen demand (BOD }(5)-1906.4 \mathrm{mg} \mathrm{L}(-1)) \text {, total Kjeldahl nitrogen } \\
\text { (TKN - } 79.8 \mathrm{mg} \mathrm{L}(-1))\end{array}$ & MI, CA & MC & GT, CT & (Kern et al. 2013) \\
\hline Sludge & $\begin{array}{l}\text { Tested } 28 \text { sewage sludge samples, Spanish wastewater treatment } \\
\text { plants }\end{array}$ & RG & MC & CT & (Roig et al., 2012) \\
\hline Wastewater & $\begin{array}{l}\text { Treated and un-treated acid mine drainage with calcinated coal } \\
\text { mining waste }\end{array}$ & RG, MI & MC & CT & (Geremias et al., 2012) \\
\hline Wastewater & $\begin{array}{l}\text { Collected from two different stations of northern India contain } \\
\text { trichloroethylene }\end{array}$ & CA & MC & GT & $\begin{array}{l}\text { (Tabrez and Ahmad, } \\
\text { 2012) }\end{array}$ \\
\hline Water & $\begin{array}{l}\text { Tested tap water treated in static conditions with gray and black } \\
\text { silicon mineral }\end{array}$ & RG & MC & CT & (Goncharuk et al., 2011) \\
\hline Dust & $\begin{array}{l}\text { Roots were exposed to cement dust over different periods at } 100 \mathrm{~m} \\
\text { from a cement factory }\end{array}$ & RG, CA, MI & MC & CT, GT & (Yahaya et al., 2012) \\
\hline Dyes & $\begin{array}{l}\text { Un-treated and bio-treated Reactive Blue } 198 \text { and triphenylmethane } \\
\text { dye }\end{array}$ & RG & MC & $\mathrm{CT} / \mathrm{TC}$ & (Balan et al., 2013) \\
\hline Fly ash & $\begin{array}{l}\text { Flay ash from thermal power plant in West Bengal } \mathrm{Zn}, \mathrm{Pb}, \mathrm{Cu}, \mathrm{Ni}, \mathrm{Cd} \\
\text { and As metals }\end{array}$ & MI, CA & MC & CT, GT & $\begin{array}{l}\text { (Chakraborty et al., } \\
\text { 2009) }\end{array}$ \\
\hline River & $\begin{array}{l}\text { Matanza-Riachuelo river sediment contains copper, lead, and } \\
\text { chromium }\end{array}$ & CA & MC & GT & (Magdaleno et al., 2008) \\
\hline Waste & $\begin{array}{l}\text { Collected from sewage effluent, agricultural and informal residential } \\
\text { runoff }\end{array}$ & RG & MC & $\mathrm{TC}$ & $\begin{array}{l}\text { (Oberholster et al., } \\
\text { 2008) }\end{array}$ \\
\hline River & $\begin{array}{l}\text { Water and sediment samples collected from the Estância Velha stream } \\
\text { of southern Brazil }\end{array}$ & RG & MC & $\mathrm{TC}$ & (Júnior et al., 2007) \\
\hline Wastewater & Wndustrial wastewater samples from Aligarh and Ghaziabad cities & $\mathrm{CA}$ & MC & GT & $\begin{array}{l}\text { (Fatima and Ahmad, } \\
\text { 2006a) }\end{array}$ \\
\hline Wasterwater & Wasterwater contains heavy metal ( $\mathrm{Cd}, \mathrm{Cr}, \mathrm{Cu}, \mathrm{Hg}, \mathrm{Pb}$ and $\mathrm{Zn}$ ) & MI & MC & CT & $\begin{array}{l}\text { (Fatima and Ahmad. } \\
\text { 2005) }\end{array}$ \\
\hline Disinfected water & $\begin{array}{l}\text { Urban wastewater disinfected with sodium hypochlorite or peracetic } \\
\text { acid }\end{array}$ & MI, CA & MC & CT, GT & (Crebelli et al., 2002) \\
\hline Wastewater & From Corumbá and Paraguay River in flood and drought seasons & MI, CA & MC & CT, GT & $\begin{array}{l}\text { (de Lima Moraes and } \\
\text { Jordao, 2001) }\end{array}$ \\
\hline Herbicide & $\begin{array}{l}\text { Herbicide ametryn encapsulated in microspheres of } \\
\text { poly(hydroxybutyrate) and poly(hydroxybutyrate-co-valerate) }\end{array}$ & & & & (da Lima et al., 2012) \\
\hline Waste & $\begin{array}{l}\text { Obtained from a sewage treatment plant, vinasse (a by-product of the } \\
\text { sugar cane industry), and a combination of both residues }\end{array}$ & $\mathrm{CA}, \mathrm{MN}$ & $\begin{array}{l}\text { MC, F } \\
\text { cell }\end{array}$ & GT, MT & $\begin{array}{l}\text { (Christofoletti et al., } \\
\text { 2013) }\end{array}$ \\
\hline Sewage sludge & $\begin{array}{l}\text { Solubilized and crude sludge from two sewage treatment stations } \\
\text { were tested }\end{array}$ & $\begin{array}{l}\text { MI, RG, CA, } \\
\text { MN }\end{array}$ & $\begin{array}{l}\mathrm{MC}, \mathrm{F} \\
\text { cell }\end{array}$ & $\begin{array}{l}\text { PT, CT, } \\
\text { GT, MT }\end{array}$ & $\begin{array}{l}\text { (Corrêa Martins et al., } \\
\text { 2016) }\end{array}$ \\
\hline Sewage sludge & $\begin{array}{l}\text { Sewage studge/soil with of } 10,25 \text { and } 50 \% \text { proportions was buried } \\
\text { for } 0,2,6 \text { and } 12 \text { months in holes }\end{array}$ & MI, CA, MN & MC & $\begin{array}{l}\text { CT, GT, } \\
\text { MT }\end{array}$ & (Mazzeo et al., 2015) \\
\hline Hair dye & $\begin{array}{l}\text { Recommended dose precisely mentioned in instructions booklet of the } \\
\text { hair dye was used }\end{array}$ & MI, CA & MC & CT, GT & (Maiti et al., 2016) \\
\hline Food dyes & $\begin{array}{l}\text { Food dyes, i.e., fast green FCF, indigo carmine, orange } \mathrm{G} \text { and tartrazine, } \\
\text { and the non-permitted dye metanil yellow }\end{array}$ & $\mathrm{MN}, \mathrm{CA}$ & MC & GT, MT & $\begin{array}{l}\text { (Roychoudhury and Giri, } \\
\text { 1989) }\end{array}$ \\
\hline Melted falling snow & $\begin{array}{l}\text { Snow samples were collected from two sites, characterized by } \\
\text { differences in pollution intensity, Belgrade, Serbia }\end{array}$ & $\mathrm{RG}, \mathrm{CA}$ & MC & TC, GT & (Blagojević et al., 2009) \\
\hline Contaminated soil & Having high level of natural radiation in the soils from Ramsar, Iran & $\mathrm{MN}, \mathrm{RG}$ & MC & TC, GT & $\begin{array}{l}\text { (Saghirzadeh et al., } \\
\text { 2008) }\end{array}$ \\
\hline Leachates & $\begin{array}{l}\text { Spent Pot Liner (aluminum industry waste, composed of organics, } \\
\text { fluoride salts, inorganic cyanides, metals, and sodium) }\end{array}$ & MI, CA & MC & CT, GT & $\begin{array}{l}\text { (Andrade-Vieira et al., } \\
\text { 2012) }\end{array}$ \\
\hline Water and sediment & From the Upper Silesia Coal Basin (USCB), Poland & MI, CA & MC & CT, GT & (Geras'kin et al., 2011) \\
\hline Industrial waste & Stones i.e., marbles and granites, includes a stage of plate polishing & MI, CA, MN & MC & $\begin{array}{l}\text { CT, GT, } \\
\text { MT }\end{array}$ & (Aguiar et al., 2016) \\
\hline
\end{tabular}


Table 2: Continue...

\begin{tabular}{|c|c|c|c|c|c|}
\hline $\begin{array}{l}\text { Contaminated water } \\
\text { and soil }\end{array}$ & $\begin{array}{l}\text { Mercuric chloride, methyl mercuric chloride, phenyl mercuric acetate } \\
\text { and a methoxy ethyl mercuric chloride based fungicide, Emisan- } 6\end{array}$ & $\mathrm{MN}, \mathrm{CA}$ & $\mathrm{MC}$ & MT, GT & (Dash et al., 1998) \\
\hline $\begin{array}{l}\text { Industrial and } \\
\text { domestic }\end{array}$ & $\begin{array}{l}\text { Effluents collected near tannery activities, } 2001 \text { and } 2002 \text { at different } \\
\text { sites along the Sapucaizinho river, Municipality of Patrocínio Paulista, } \\
\text { State of São Paulo, Brazil }\end{array}$ & MI, CA, MN & $\mathrm{MC}$ & $\begin{array}{l}\text { CT, GT, } \\
\text { MT }\end{array}$ & $\begin{array}{l}\text { (Matsumoto and Marin- } \\
\text { Morales, 2004) }\end{array}$ \\
\hline River and well water & Alamuyo River in Ibadan, Nigeria and wells alongside river & MI, CA & MC & CT, GT & (Fawole et al., 2008) \\
\hline Radiation & Roots were exposed to radiofrequency electromagnetic fields & $\mathrm{MI}, \mathrm{CA}$ & MC & CT, GT & (Tkalec et al., 2009) \\
\hline Wastewater & $\begin{array}{l}\text { Un-treated and physical, chemical, and biologically treated acid mine } \\
\text { drainage wastewater }\end{array}$ & MI & MC & CT & (Defaveri et al., 2009) \\
\hline Textile effluents & $\begin{array}{l}\text { Un-treated and treated at the absorbed doses of 5-15 kGy in } \\
\text { combination with } \mathrm{H}_{2} \mathrm{O}_{2}\end{array}$ & RG, MI & MC & CT & (Iqbal and Nisar, 2015) \\
\hline Textile dye & $\begin{array}{l}\text { Treated and un-treated azo dye Remazol orange by Pseudomonas } \\
\text { aeruginosa BCH in plain distilled water }\end{array}$ & RG & MC & CT & (Jadhav et al., 2012) \\
\hline Leachate & $\begin{array}{l}\text { Raw and simulated e-waste leachates (Fe, } \mathrm{Cd}, \mathrm{Mn}, \mathrm{Cu}, \mathrm{Ni}, \mathrm{Cr} \mathrm{As}, \mathrm{Zn} \text { and } \\
\mathrm{Pb} \text { metals) }\end{array}$ & MI, CA & MC & CT, GT & (Bakare et al., 2012) \\
\hline Tannery effluents & $\begin{array}{l}\text { Composition: } \mathrm{pH}(8.5), \mathrm{EC}(11.94 \mathrm{dSm}-1), \mathrm{BOD}(499 \mathrm{mg} / \mathrm{L}), \mathrm{COD}(1382 \\
\mathrm{mg} / \mathrm{L}) \text { and } \mathrm{Cr} \text { content }(2.32 \mathrm{mg} / \mathrm{L})\end{array}$ & MI, CA, MN & $\mathrm{MC}$ & $\begin{array}{l}\text { CT, GT, } \\
\text { MT }\end{array}$ & (Gupta et al., 2012) \\
\hline River water & Water samples from river Rasina, Serbia from nine different places & MI, CA & $\mathrm{MC}$ & CT, GT & (Vujošević et al., 2008) \\
\hline Air pollution & $\begin{array}{l}\text { Roots were exposed to field conditions at six research plots in the } \\
\text { most polluted areas, Slovenia }\end{array}$ & MI, CA & $\mathrm{MC}$ & CT, GT & (Glasenčnik et al., 2004) \\
\hline Air pollution & $\begin{array}{l}\text { Roots were exposed at eight sampling plots in the vicinity of major } \\
\text { Slovene local emission sources }\end{array}$ & MI, CA & MC & CT, GT & (Glasencnik et al., 2002) \\
\hline Contaminated soil & Soil contaminated with Diuron (herbicide) & MI, CA, MN & $\mathrm{MC}$ & $\begin{array}{l}\text { CT, GT, } \\
\text { MT }\end{array}$ & (Saxena et al., 2004) \\
\hline Waste & Diluted and un-diluted waste drilling fluid samples & MI, CA & $\mathrm{MC}$ & $\begin{array}{l}\text { CT, TC, } \\
\text { GT }\end{array}$ & (Vidaković et al., 1993) \\
\hline Sea water & $\begin{array}{l}\text { Roots were exposed to ballast water at } 0.5 \%, 1 \%, 5 \% \text {, and } 10 \% \\
\text { concentrations }\end{array}$ & MI, CA & MC & CT, GT & (Olorunfemi et al., 2012) \\
\hline River water & $\begin{array}{l}\text { Samples collected from Shiroka, Zogaj, Shegan, Kamicë, Stërbeq, Buna } \\
\text { bridge, Bahçellek, Zues and Dajç (Shkodra lake, Buna and Drini rivers) }\end{array}$ & MI, CA & $\mathrm{MC}$ & CT, GT & (Kopliku et al., 2012) \\
\hline Sewage effluent & collected form Mandsaur (Madhya Pradesh), India & RG & MC & $\mathrm{CT} / \mathrm{TC}$ & $\begin{array}{l}\text { (Shashank and Suresh, } \\
\text { 2013) }\end{array}$ \\
\hline Leachate & $\begin{array}{l}\text { Raw and simulated leachate from a rural refuse dump site at Odo Oba, } \\
\text { South-West Nigeria }\end{array}$ & MI, CA & MC & CT, GT & (Bakare, 2001) \\
\hline Wastewater & $\begin{array}{l}\text { Wastewaters from two pharmaceutical production processes, } \\
\text { cotrimoxazole B wastewater and Piriton wastewater }\end{array}$ & MI, CA & MC & CT, GT & (Lateef, 2007) \\
\hline Wastewater & Water from Dobër wells, Vraka runnel, and Shegan waterside & MI, RG & $\mathrm{MC}$ & CT & $\begin{array}{l}\text { (Mesi and Kopliku, } \\
\text { 2011) }\end{array}$ \\
\hline Wastewater & $\begin{array}{l}\text { Pharmaceutical effluent at } 0.5,1,2.5,5 \text { and } 10 \% \text {; and } 1,5,10,25 \text { and } \\
50 \% \text { concentrations }\end{array}$ & MI, RG, CA & MC & CT, GT & (Bakare et al., 2009) \\
\hline Wastewater & electroplating wastewater ( metal ions and cyanides) & $\mathrm{CA}$ & MC & GT & $\begin{array}{l}\text { (Somashekar and Arekal, } \\
\text { 1983) }\end{array}$ \\
\hline Wastewater & $\begin{array}{l}\text { Bio-treated and un-treated bleaching effluent from two kraft pulp } \\
\text { mills }\end{array}$ & $\mathrm{MI}, \mathrm{CA}$ & MC & CT, GT & $\begin{array}{l}\text { (Chaparro and Pires, } \\
\text { 2011) }\end{array}$ \\
\hline Leachate & $\begin{array}{l}\text { Limeira Sanitary LandfillBrazilian state of São Paulo (Al, Cd, Pb, Cu, Cr, } \\
\mathrm{Ni}, \mathrm{Zn}, \mathrm{K}, \mathrm{Na}, \mathrm{Fe}, \mathrm{Mn} \text { and pH 8.01, Color - Abs. }(400 \mathrm{~nm}) 2.46, \mathrm{Turbidity} \\
\text { (UT) 68.70, Condutivity }(\mathrm{mS}) 12.21,5 \mathrm{Hardness}(\mathrm{mg} / \mathrm{L} \mathrm{CaCO} 3) 934.00 \text {, } \\
\left.\text { TOC }(\mathrm{mg} / \mathrm{L}) 1116.10, \mathrm{AOX}(\mu \mathrm{g} / \mathrm{L}) 690.70, \mathrm{NH}^{4+}(\mathrm{mg} / \mathrm{L}) 426.00\right)\end{array}$ & $\mathrm{MI}, \mathrm{RG}$ & MC & $\mathrm{CT} / \mathrm{TC}$ & (Pelegrini et al., 2007) \\
\hline Tap water & $\begin{array}{l}\text { Borehole water supply in six halls of residence of the University of } \\
\text { Benin main campus }\end{array}$ & MI, CA & MC & CT, GT & (Olorunfemi, 2013) \\
\hline Wastewater & $\begin{array}{l}\text { Advanced oxidation treated and un-treated wastewater produced by } \\
\text { the process of rhodamine B dyeing }\end{array}$ & MI, CA & MC & CT, GT & (Machado et al., 2012) \\
\hline Water sample & $\begin{array}{l}\text { Nën-Shkodra lowland agricultural area after massive 2010-2011 } \\
\text { flooding }\end{array}$ & MI, CA, RG & MC & CT, GT & (Kopliku and Mesi 2012) \\
\hline Textile dye & Un-treated and bio-treated reactive Navy Blue RX dye & $\mathrm{MI}, \mathrm{CA}$ & MC & CT, GT & (Watharkar et al., 2013) \\
\hline Textile dye & Un-treated and bio-treated reactive blue 198 & MI & MC & $\mathrm{CT}$ & $\begin{array}{l}\text { (Poonkuzhali et al., } \\
\text { 2011) }\end{array}$ \\
\hline River water & River Vale do Jamari, brazil & $\mathrm{MI}, \mathrm{MN}, \mathrm{CA}$ & MC & $\begin{array}{l}\text { CT, GT, } \\
\text { MN }\end{array}$ & $\begin{array}{l}\text { (de Oliveira Meneguetti } \\
\text { et al., 2012) }\end{array}$ \\
\hline Mixture & $\begin{array}{l}\text { Samples collected from outlets of sewage effluent, agricultural and } \\
\text { informal residential runoff }\end{array}$ & RG, MI & MC & CT & $\begin{array}{l}\text { (Oberholster et al., } \\
\text { 2008) }\end{array}$ \\
\hline Food industry & Monosodium glutamate used as flavor enhancer in foods & RG, MI, CA & MC & $\mathrm{CT}, \mathrm{MT}$ & $\begin{array}{l}\text { (Adeyemo and } \\
\text { Farinmade, 2016) }\end{array}$ \\
\hline
\end{tabular}


Table 2: Continue...

\begin{tabular}{|c|c|c|c|c|c|}
\hline Waste & $\begin{array}{l}\text { Surface water from five sites of Hooghly River in West Bengal, India, } \\
\text { along the banks of which many shipbuilding and scrap industries }\end{array}$ & MN, MI, CA & MC & $\begin{array}{l}\text { CT, GT, } \\
\text { MT }\end{array}$ & (Singh et al., 2014) \\
\hline Water sample & $\begin{array}{l}\text { Sodium metabisulfite in sea waters and sediments collected in a } \\
\text { shrimp farm in Cajueiro da Praia (Luis Correia), state of Piauí, Brazil, }\end{array}$ & MN, CA, MI & MC & TC & (Matos et al., 2011) \\
\hline Wastewater & $\begin{array}{l}\text { Upflow Immobilized Biomass Anaerobic Reactor treated and un- } \\
\text { treated hospital wastewater }\end{array}$ & MI,RG & MC & $\mathrm{CT} / \mathrm{TC}$ & $\begin{array}{l}\text { (Porras Torres et al., } \\
\text { 2013) }\end{array}$ \\
\hline Food industry & $\begin{array}{l}\text { Food preservatives (sodium benzoate and sodium metabisulphite) } \\
\text { were tested at different concenrations }\end{array}$ & RG, MI, CA & MC & CT, GT & (Onyemaobi et al., 2011) \\
\hline Wastewater & Chemical industrial effluent & CA & MC & $\mathrm{CT}$ & (Xing et al., 1995) \\
\hline Waste sludge & Raw and biosolid sludge by diplopods & CA & MC & GT & $\begin{array}{l}\text { (Christofoletti et al., } \\
\text { 2012) }\end{array}$ \\
\hline Leachate & $\begin{array}{l}\text { Raw municipal landfill leachates from Radiowo municipal Poland and } \\
\text { zeolite treated leachate }\end{array}$ & $\mathrm{RG}, \mathrm{CA}$ & MC & PT, GT & $\begin{array}{l}\text { (Obidoska and Jasińska, } \\
\text { 2008) }\end{array}$ \\
\hline Wastewater & $\begin{array}{l}\text { Petroleum refinery wastewater tested at } 20 \%, 40 \% .60 \%, 80 \% \text { and } \\
100 \% \text { concentrations }\end{array}$ & CA, MI & MC & CT, GT & (Obute et al., 2005) \\
\hline Wastewater & Raw and treated effluents of dairy industry & CA, MI & MC & CT, GT & Olorunfemi et al. (2012) \\
\hline River water & $\begin{array}{l}\text { Santa Maria da Vitória River, Brazil, received domestic, municipal and } \\
\text { agricultural waste }\end{array}$ & CA & MC & GT & (Grippa et al., 2012) \\
\hline Food industry & $\begin{array}{l}\text { Dye orange red (amalgamation of two primary food colourants like } \\
\text { caramoisine and sunset yellow) was studied }\end{array}$ & CA & MC & GT & (Tripathy et al., 2015) \\
\hline Soil sample & $\begin{array}{l}\text { Un-treated and treated soil from a site heavily contaminated with } \\
\text { polychlorinated biphenyls }\end{array}$ & CA & MC & GT & (Meier et al., 1997) \\
\hline Food industry & $\begin{array}{l}\text { Chitosan and poly(methacrylic acid) nanoparticles (size } 60,82 \text {, and } \\
111 \mathrm{~nm} \text { ) were studied, which are used for food packing }\end{array}$ & MI & MC & CT & (De Lima et al., 2010) \\
\hline Wastewater & $\begin{array}{l}\text { Un-treated and treated textile wastewater with yeast cell biomass } \\
\text { and S. cerevisiae MTCC } 463\end{array}$ & RG, MI, CA & MC & $\begin{array}{l}\text { PT, CT, } \\
\text { GT }\end{array}$ & (Phugare et al., 2010) \\
\hline Pyrolysis oil & Oils produced from different sources (poplar, beech and spruce) & $\mathrm{MI}, \mathrm{CA}$ & MC & CT, GT & (Holan et al., 2014) \\
\hline Sludge & Oil refinery sludge tested at different concentrations & MI, CA & MC & CT, GT & (Tanti et al., 2009) \\
\hline Water sample & $\begin{array}{l}\text { São Gonçalo Channel, water supply source of the city of Pelotas, Brazil } \\
\text { and the surrounding region }\end{array}$ & MI, CA & MC & CT, GT & (Paiva et al., 2008) \\
\hline Water sample & $\begin{array}{l}\text { Rural settlements area (Obazuwa community in Ovia North East Local } \\
\text { Government Area of Edo State, Nigeria) }\end{array}$ & MI, CA, MN & MC & $\begin{array}{l}\text { CT, GT, } \\
\text { MT }\end{array}$ & (Olorunfemi et al., 2014) \\
\hline Tannery wastewater & Tannery effluent treated with Aqueous leaf extract & RG, MI & MC & CT & $\begin{array}{l}\text { (Poonkuzhali et al., } \\
\text { 2013) }\end{array}$ \\
\hline Wastewater & $\begin{array}{l}\text { Advanced oxidation process (ozone and ozone/UV) treated and un- } \\
\text { treated kraft cellulose pulp mill }\end{array}$ & CA & MC & GT & (Chaparro et al., 2015) \\
\hline Soil samples & $\begin{array}{l}\text { Collected near copper-smelters (the Middle Urals) and Ni-enriched } \\
\text { soil from an area of a natural geochemical anomaly (the Polar Urals) }\end{array}$ & CA, MI, MN & MC & $\begin{array}{l}\text { CT, GT, } \\
\text { MT }\end{array}$ & (Kataeva et al., 2012) \\
\hline Textile dye & Un-treated and treated malachite green with laccase enzyme & RG, MI & MC & $\mathrm{TC}$ & Balan et al. (2012) \\
\hline Wastewater & Un-treated and treated hospital effluent by ozonation & RG & MC & $\mathrm{TC}$ & $\begin{array}{l}\text { (Grisales Penagos et al., } \\
\text { 2012) }\end{array}$ \\
\hline Textile dyes & $\begin{array}{l}\text { Un-treated and calcium-alginate pectin entrapped bitter gourd } \\
\text { peroxidases treated disperse Brown } 1 \text { and Disperse Red } 17 \text { dyes }\end{array}$ & RG/MI & MC & $\mathrm{TC} / \mathrm{CT}$ & (Satar and Husain, 2011) \\
\hline Waste & $\begin{array}{l}\text { Sample contaminated with } \mathrm{Cd}^{2+}, \mathrm{Cr}^{6+}, \mathrm{Cu}^{2+}, \mathrm{Ni}^{2+}, \mathrm{Pb}^{2+}, \mathrm{BHC}, 2,4-\mathrm{D} \text {, } \\
\text { mancozeb and phenols }\end{array}$ & RG, MI & MC & $\mathrm{CT} / \mathrm{TC}$ & $\begin{array}{l}\text { (Khan and Ahmad } \\
(2006)\end{array}$ \\
\hline Textile dye & $\begin{array}{l}\text { Treated and un-treated remazol red (a monochloro sulphonated azo } \\
\text { dye) by Pseudomonas aeruginosa } \mathrm{BCH}\end{array}$ & CA & MC & GT & Jadhav et al. (2011) \\
\hline Distillery waste & $\begin{array}{l}\text { Roots were exposed to distillery effluent }(25,50,75 \text { and } 100 \%) \\
\text { wastewater }\end{array}$ & & & & $\begin{array}{l}\text { (Hemanth Kumar et al. } \\
\text { 2015) }\end{array}$ \\
\hline Sugar industry & $\begin{array}{l}\text { Vinasse (by-product of the biomass distillation, mainly for the } \\
\text { production of ethanol, from different cultures such as sugarcane }\end{array}$ & MI, CA, MN & MC & $\begin{array}{l}\text { CT, GT, } \\
\text { MT }\end{array}$ & $\begin{array}{l}\text { (Pedro-Escher et al., } \\
\text { 2016) }\end{array}$ \\
\hline River water & $\begin{array}{l}\text { Vistula river water at selected points in Warsaw (downstream from } \\
\text { Gruba KaĞka) (Poland) }\end{array}$ & MI, GT & MC & CT, GT & (Obidoska et al., 2013) \\
\hline Pulp and paper & $\begin{array}{l}\text { Treated the paper and pulp industry effluent by Rhodococcus sp. NCIM } \\
2891 \text { and un-treated wastewater }\end{array}$ & RG, MI & MC & $\mathrm{CT} / \mathrm{TC}$ & (Nadaf et al., 2014) \\
\hline Fingicide & $\begin{array}{l}\text { Fungicide tilt in root tip cells of A. cepa. Bulbs were exposed to } 0.02 \% \text {, } \\
0.04 \%, 0.06 \%, 0.08 \% \text { ) concentrations of tilt for } 3,6,9,12 \mathrm{~h}\end{array}$ & MI, CA & MC & CT, GT & (Pulate et al., 2014) \\
\hline Water sample & $\begin{array}{l}\text { Roots were exposed to five different water samples collected from } \\
\text { boreholes Ring road, Sakponba, Ekosodin, Science and Eddy Grace in } \\
\text { Benin City, Edo State, Nigeria for 96-h }\end{array}$ & MI, CA & MC & CT, GT & (Adelanwa et al., 2014) \\
\hline
\end{tabular}


Table 2: Continue...

\begin{tabular}{|c|c|c|c|c|c|}
\hline Nanoparticles & $\begin{array}{l}\text { Different sizes silica nanoparticles (SiNP) (TM40 (22 nm), HS30 (12 } \\
\mathrm{nm}), \mathrm{SM} 30(7 \mathrm{~nm}) \text { with concentrations ranging from } 0.19 \text { to } 163.8 \mathrm{~g} / \mathrm{L} \\
\text { (TM40) and } 0.29 \text { to } 122.85 \mathrm{~g} / \mathrm{L} \text { (HS30 and SM30) were tested }\end{array}$ & MI & MC & $\mathrm{CT}$ & (Silva, 2014) \\
\hline Wastewater & Petrochemicals, air liquid and polyester resin effluents & $\mathrm{MI}, \mathrm{CA}$ & MC & CT, GT & (Abu et al., 2015) \\
\hline Waste & $\begin{array}{l}\text { Complex environmental mixtures (surface water or industrial } \\
\text { wastewater) }\end{array}$ & & & & (Teena et al., 2016) \\
\hline Water sample & Collected from from a regional radioactive waste repository & $\mathrm{MI}, \mathrm{CA}$ & MC & CT, GT & (Pyatkova et al., 2009) \\
\hline Wastewater & $\begin{array}{l}\text { Bio-treatment of industrial effluent containing having eleveated values } \\
\text { water parameters i.e., COD, color and heavy metals }\end{array}$ & RG & MC & $\mathrm{CT}$ & (Patel et al,, 2015) \\
\hline $\begin{array}{l}\text { Contaminated sea } \\
\text { water }\end{array}$ & $\begin{array}{l}\text { Bilge water (water from oceanic vessels is usually discharged through } \\
\text { the bilge wells), contains } \mathrm{Cu}, \mathrm{Mn}, \mathrm{Pb}, \mathrm{Fe}, \mathrm{Cd}, \mathrm{Cr}, \mathrm{Ag}, \mathrm{Ni} \text { and } \mathrm{Zn} \text { heavy } \\
\text { metals }\end{array}$ & MI, CA & MC & CT, GT & (Olorunfemi et al., 2015) \\
\hline Pesticides & $\begin{array}{l}\text { Emulsifiable concentrate of cypermethrin, deltamethrin, } \\
\text { lambdacyhalothrin and endosulfan at } 1.0,5.0,10.0,20.0 \text { and } 40.0 \mathrm{ppm} \\
\text { concentrations for } 48-72 \mathrm{~h} \text { exposure }\end{array}$ & $\mathrm{MI}, \mathrm{CA}$ & MC & CT, GT & $\begin{array}{l}\text { (Yekeen and Adeboye, } \\
\text { 2013) }\end{array}$ \\
\hline Textile wastewater & $\begin{array}{l}\text { textile effluent on A. test at } 1,2,5,10,25,50 \text { and } 100 \%(\mathrm{v} / \mathrm{v} \text {; } \\
\text { effluent/tap water) concentrations for } 96 \mathrm{~h} \text {, contains } \mathrm{Fe}, \mathrm{Cd}, \mathrm{Mn}, \mathrm{Ni}, \mathrm{Cr} \text {, } \\
\text { and other disturbed water quality parameters }\end{array}$ & MI, CA & MC & CT, GT & (Alimba et al., 2013) \\
\hline $\begin{array}{l}\text { Insecticides and } \\
\text { fungicide }\end{array}$ & $\begin{array}{l}\text { Insecticides (Endosri \& Nuvan) and fungicide (Kvistin) at different } \\
\text { concentrations }\end{array}$ & RG, MI & MC & CT & (Kuchy et al., 2015) \\
\hline Wastewater & $\begin{array}{l}\text { Un-treated and treated effluents with aquatic macrophytes Salvinia } \\
\text { auriculata Aublet, Mexico }\end{array}$ & RG & MC & CT & (Gonçalves et al., 2015) \\
\hline Textile dyes & $\begin{array}{l}\text { Un-treated and bio-treated textile dyes using peroxidases from turnip } \\
\text { roots }\end{array}$ & RG, MI & MC & CT, PT & $\begin{array}{l}\text { (Kulshrestha and } \\
\text { Husain, 2007) }\end{array}$ \\
\hline electronic waste & $\begin{array}{l}\text { From Iloabuchi electronic market, Diobu, Rivers State, Nigeria tested } \\
\text { at } 5 \%, 10 \%, 25 \%, 50 \% \text {, and } 100 \% \text { concentrations }\end{array}$ & $\mathrm{MI}, \mathrm{CA}$ & MC & CT, GT & $\begin{array}{l}\text { (Babatunde and } \\
\text { Anabuike, 2015) }\end{array}$ \\
\hline Hospital waste & $\begin{array}{l}\text { Treated hospital effluent applying ozone at different } \mathrm{pH} \text { conditions } \\
(3,0,6,7,10)\end{array}$ & RG, MI & MC & CT & (Dayana et al., 2012) \\
\hline Contaminated soil & Four soil types collected from different regions of Amritsar, India & CA & MC & GT & (Soodan et al., 2014) \\
\hline Effluents & Effluents arising from the ornamental rock polishing & MI, CA & MC & CT, GT & (Teixeira et al., 2015) \\
\hline Textile effluents & $\begin{array}{l}\text { Un-treated and bio-treated effluents from Masood Textile, Kalash } \\
\text { Textile, Khyber Textile and Sitara Textile (Faisalabad, Pakistan) }\end{array}$ & MI, RG & MC & CT & (Bilal et al., 2016) \\
\hline Contaminated water & $\begin{array}{l}\text { Marrakesh's CMR wastewater, Draa Lasfar mine located about } 12 \mathrm{~km} \\
\text { south-West of the city of Marrakech, Morocco }\end{array}$ & MI, CA & MC & CT, GT & (Chaik et al., 2011) \\
\hline Water sample & Orathupalayam dam Water, India & MI, CA & MC & CT, GT & $\begin{array}{l}\text { Gajalakshmi and Ruban } \\
\text { (2014) }\end{array}$ \\
\hline Waste & Metalworking fluids at $1 / 250,1 / 500$ and $1 / 1000$ concentrations & MI, CA, MN & MC & $\begin{array}{l}\text { CT, GT, } \\
\text { MT }\end{array}$ & (Pekol, 2014) \\
\hline Agriculture & $\begin{array}{l}\text { Abamectin+Emaamectin benzoate) at } 0,0.3,0.4,0.5,0.6,0.7,0.8,0.9 \\
\text { and } 1 \mathrm{ml} / \mathrm{L} \text { concentrations for } 24 \mathrm{~h} \text { exposure }\end{array}$ & $\mathrm{MI}, \mathrm{CA}, \mathrm{MN}$ & MC & $\begin{array}{l}\text { CT, GT, } \\
\text { MT }\end{array}$ & (Ahmed, 2014) \\
\hline Wastewater & $\begin{array}{l}\text { Dandugan Oya, a water canal located in the Western Province of Sri } \\
\text { Lanka receives industrial waste from multiple sources }\end{array}$ & $\begin{array}{l}\text { MI, CA, NA, } \\
\text { MN }\end{array}$ & MC & $\begin{array}{l}\text { CT, GT, } \\
\text { MN }\end{array}$ & $\begin{array}{l}\text { (Kannangara and } \\
\text { Pathiratne, 2015) }\end{array}$ \\
\hline $\begin{array}{l}\text { Electroplating } \\
\text { industry }\end{array}$ & $\begin{array}{l}\text { Wastewater from Madurai city, India contains heavy metal }(\mathrm{Ni}, \mathrm{Cu}, \mathrm{Fe} \text {, } \\
\mathrm{Mn} \text { and } \mathrm{Pb} \text { ) higher than permissible limits }\end{array}$ & MI, CA & MC & CT, GT & (Nagarajan et al., 2014) \\
\hline Contaminated water & $\begin{array}{l}\text { Arsenic contaminated groundwater from Eastern region of Burdwan } \\
\text { district, West Bengal }\end{array}$ & MI & MC & $\mathrm{CT}$ & (Bandyopadhyay, 2015) \\
\hline Nanoparticles & $\mathrm{Al}_{2} \mathrm{O}_{3}$ nanoparticles at $0.01,0.1,1,10$, and $100 \mu \mathrm{g} / \mathrm{mL}$ concentrations & $\mathrm{MI}, \mathrm{CA}$ & MC & CT, GT & (Rajeshwari, 2015) \\
\hline Wastewater & $\begin{array}{l}\text { Sediment elutriate from Tambis River, Barobo, Surigao Del Sur, } \\
\text { Philippines, } 1000 \mathrm{~g} / \mathrm{L} \text { and } 500 \mathrm{~g} / \mathrm{L} \text {, for } 72 \text { h exposure }\end{array}$ & MI, CA & MC & CT, GT & (Fajardo et al., 2015) \\
\hline Water sample & Ballast water at $0.5,1,5$ and $10 \%$ concentrations & CA & MC & GT & (Olorunfemi et al., 2014) \\
\hline Water sample & Different concentrations of bilge water $(1,5,10,25$ and $50 \%)$ & & & & (Olorunfemi et al., 2015) \\
\hline Food industry & $\begin{array}{l}\text { Liquid waste produced in the process of industrialization of the bitter } \\
\text { cassava (a plant used as food and an ingredient in industry) }\end{array}$ & MI, CA & MC & CT, GT & (Viana et al., 2014) \\
\hline Fly ash & Un-treated and vermicomposted flyash & MI, RG & MC & CT & (Sarojini et al., 2010) \\
\hline Wastewater & $\begin{array}{l}\text { Irrigation water used for vegetables in a Greater São Paulo, Brazil } \\
\text { watershed region }\end{array}$ & CA, MI, MN & MC & GT & $\begin{array}{l}\text { (de Maio Lacerda et al., } \\
\text { 2014) }\end{array}$ \\
\hline Sugar industry & $\begin{array}{l}\text { Raw and vermicomposted (Eisenia fetida + cattle dung) sugar beet } \\
\text { mud and pulp (waste by-products of the sugar industry) }\end{array}$ & CA & MC & GT & (Bhat et al., 2015) \\
\hline Contaminated water & Well water from Vazante-MG city, Brazil (zinc producer district) & CA & MC & GT & (da Silva et al., 2013) \\
\hline Food industry & $\begin{array}{l}\text { Synthetic Cheese and Cheddar Cheese (food flavors) at } 1.0 \text { and } 2.0 \mathrm{~mL} \\
\text { doses }\end{array}$ & MI & MC & $\mathrm{CT}$ & (Moura et al., 2016) \\
\hline
\end{tabular}


Table 2: Continue...

\begin{tabular}{|c|c|c|c|c|c|}
\hline Leachate & $\begin{array}{l}\text { Gaseous emissions (biogas) from three municipal landfills and } \\
\text { Leachate samples }\end{array}$ & MI, CA & MC & CT, GT & (Feretti et al., 2008) \\
\hline Textile industry & $\begin{array}{l}\text { Un-treated and treated textile effluent using immobilized manganese } \\
\text { peroxidase }(\mathrm{MnP}) \text { and detoxification }\end{array}$ & MI & MC & $\mathrm{CT}$ & (Bilal et al., 2016a) \\
\hline Textile industry & $\begin{array}{l}\text { Un-treated and Ganoderma lucidum IBL- } 05 \mathrm{MnP} \text { immobilized on Sol- } \\
\text { Gel matrix treated textile effluent }\end{array}$ & MI & MC & CT & (Bilal et al., 2016b) \\
\hline Textile dyes & Un-treated and Agar-agar gel immobilized MnP treated dyes & MI & MC & CT & (Bilal et al., 2016c) \\
\hline Textile industry & $\begin{array}{l}\text { Un-treated and Ganoderma lucidum IBL- } 05 \text { crude ligninolytic enzymes } \\
\text { (MnP LiP and Laccase) treated textile effluents }\end{array}$ & MI & MC & CT & (Bilal et al., 2016d) \\
\hline Textile dye & Un-treated and $\mathrm{N}-\mathrm{TiO}_{2}$ nanostructure treated textile dye & MI, CA & MC & CT, GT & (Kadam et al., 2014) \\
\hline Wastewater & $\begin{array}{l}\text { Refined petroleum products (Petrol, Kerosene) and locally made soft } \\
\text { drink (Zobo) at different concentration were tested }\end{array}$ & MI, CA, MN & MC & $\begin{array}{l}\text { CT, GT, } \\
\text { MT }\end{array}$ & (Obute et al., 2016) \\
\hline Coal mine waste & $\begin{array}{l}\text { used calcinated coal mining waste to treat river water contaminated } \\
\text { by residues derived from coal mines from the Urussanga River } \\
\text { (Urussanga, Santa Catarina state, Brazil) }\end{array}$ & MI & MC & CT & $\begin{array}{l}\text { (da Silva Francisconi et } \\
\text { al., 2013) }\end{array}$ \\
\hline Textile dye & Un-treated and UV/TiO2/ $\mathrm{H}_{2} \mathrm{O}_{2}$ treated Disperse Red F3BS & RG, MI & MC & $\mathrm{CT}$ & (Bokhari et al., 2015) \\
\hline Surfactant & $\begin{array}{l}\text { Un-treated and gamma radiation } / \mathrm{H}_{2} \mathrm{O}_{2} \text { treated nonylphenol } \\
\text { ethoxylates }\end{array}$ & $\mathrm{RG}, \mathrm{MI}$ & MC & CT & (Iqbal et al., 2015) \\
\hline River water & $\begin{array}{l}\text { Todos os Santos River (TSR), Brazil receives a high discharge of } \\
\text { untreated effluents from various sources }\end{array}$ & $\mathrm{CA}, \mathrm{MN}$ & MC & GT, MT & (Blanc et al., 2013) \\
\hline
\end{tabular}

The metallurgy industries discharge a variety of toxic heavy metals like $\mathrm{Zn}, \mathrm{Cd}, \mathrm{Cr}, \mathrm{Cu}, \mathrm{Ni}, \mathrm{Pb}, \mathrm{V}$, Mo, Co along with other solid waste and is threat to the environment, if improperly managed (Lee and Pandey, 2011). The major waste generation units include smelter flue dust, converter slag, dump slag, tailings, smelting slag, filter press residue, wastesludge, red mud, metallurgical-waste dump, pyrite sludge spillage, waste and blast furnace slag and sludge in large amount. For example, $300-400 \mathrm{~kg}$ of slag is produced in the production of one ton of pig iron and a single aluminum (Al) manufacturing industries generate about 35,000 ton of spent potliners (waste) per annum (Andrade et al., 2008; Kumar et al., 2006).

To study the toxicity of metallurgical wastes and metals, various authors reported the sensitivity of A. cepa. While studying the aluminum manufacturing waste,spent potliners waste was found to be toxic to A. cepa root cells (Andrade et al., 2008). A strong inhibition of root growth at higher spent potliners concentrations has been observed. The CA's such as bridges, fragments, stickiness, multipolar anaphase, later segregation and cell death was reported as a result of spent potliners exposure. Other studies also revealed the CA induction and decrease in MI when root were exposed to aluminum metal solution (Fiskesjo, 1988; Roy et al., 1989).

The cytogenetic effect of $\mathrm{Cu}$ mine waste at different concentrations $(10 \%, 75 \%$ and $100 \%)$ was also studied using CA endpoint in A. cepa root assays (Inceer et al., 2000). Authors recorded a dose-dependent relationship with the inhibition of MI and at $100 \%$ concentration the highest cytotoxicity was recorded. The authors also reported the chromosomal breaks, bridges and adherences. They concluded that $\mathrm{Cu}$ wastes can cause deleterious effects on $A$. cepa root cells. While studying the genotoxic effects of leachates from solid waste of a metal industry (Chandra et al.,
2005), reported the CA, MI and MN alterations in the A. cepa root cells. The A. cepa bulbs were exposed for $48 \mathrm{~h}$ to 2.5 $10 \%$ leachates wastes and resultantly, significant inhibition of MI, induction of CA, mitotic aberrations and MN formation were found in all experimental groups exposed to metal waste leachate. The toxic effects were found concentration dependent and MI was severely inhibited at $10 \%$ leachate aqueous solution exposure. These toxic responses may have relied on raised heavy metals $(\mathrm{Cr}, \mathrm{Ni}$ and $\mathrm{Fe})$ concentrations in leachates wastes. The CA inductions and root growth inhibition in A. cepa root exposed to the metallic industry wastewater for $24 \mathrm{~h}$ was also observed (Nielsen and Rank, 1994).

Few studies also highlighted the individual effects of metal on CA's, MI, NA's and MN's abnormalities. For example, stickiness, C-mitosis, deformed nuclei and decrease in the MI is correlated with $\mathrm{Cu}$ metal and $\mathrm{Mn}$ with sticky chromosomes (Abdel Migid et al., 2007; Fiskesjo, 1988). In the presence of Zn, MI inhibition and sticky chromosomes, C-metaphases, bridges and metaphase disturbed by lagging chromosomes were observed (El-Ghamery et al., 2003) and Cd induced chromatin fragmentation, changes in the nucleolus and ultimately cell death (Andrade et al., 2008), while $\mathrm{Pb}$ reduced root growth and caused mitotic irregularities, including Cmitosis, anaphase bridges and chromosome stickiness. Furthermore, MN formation during interphase, irregularly shaped nuclei and nuclei with decomposed nuclear material were observed in the presence of $\mathrm{Pb}$ (Behboodi and Samadi, 2004; Liu et al., 1994). Analyses of $A$. cepa meristematic cells exposed to water samples with high $\mathrm{Ni}$ and $\mathrm{Pb}$ levels, indicated the CA's abnormalities (Bianchi et al., 2011).

Recently, the use of nanoparticles in various field has attracted the attention (Kumari et al., 2009b) and in this regard, the cytotoxic and genotoxic impacts of silver nanoparticles using root tip cells of $A$. cepa have been investigated. The A. cepa root tips were treated with four 
different concentrations $(25,20,75$, and $100 \mathrm{ppm})$ of size $<100 \mathrm{~nm}$ and studied the MI, distribution of cells in mitotic phases, various types of CA's such as disturbed metaphase, sticky chromosome, cell wall disintegration and breaks. The MI value was $27.62 \%$ in treated group of plants as compared to control $(60.3 \%)$ and with increasing concentration of the nanoparticles decrease in the mitotic index was noticed. The authors revealed that silver nanoparticles could penetrate plant system and may impair stages of cell division causing chromatin bridge, stickiness, disturbed metaphase, multiple chromosomal breaks and cell disintegration and recommendedtthe importance of $A$. cepa in the ecosystems for evaluation of toxicological impact of the nanoparticles in the environment. The $\mathrm{ZnO}$ nanoparticles were also revealed to have cytogentic effects (Kumari et al., 2011). A. cepa roots were treated with the dispersions of $\mathrm{ZnO}$ NPs at four different concentrations $(25,50,75$, and $100 \mu \mathrm{g} / \mathrm{mL})$. The MI value decreased along with significant inductions of MN and CA's. Results demonstrated the clastogenic/genotoxic and cytotoxic nature of ZnO NPs . Therefore, A. cepa cytogenetic test is suggested to evaluate the genotoxicity nanomaterials. Similarly, $\mathrm{Al}_{2} \mathrm{O}_{3}$ nanoparticles $\left(\mathrm{Al}_{2} \mathrm{O}_{3} \mathrm{NPs}\right)$ were also investigated for their cytogenetic potential in $A$. cepa root tip cells at $0.01,0.1,1,10$, and $100 \mu \mathrm{g} / \mathrm{mL}$ concentrations (Rajeshwari et al., 2015). MI value reduced in dosedependent manner (42 to $28 \%$ ) along with CA's (sticky, multipolar and laggard chromosomes, chromosomal breaks, and the formation of binucleate cells). The interaction between roots and NP's were studied by optical, fluorescence, confocal laser scanning microscopy and FT-IR techniques. In another study, toxicity of cobalt and zinc oxide NPs using growth, root and cell morphology enpoints was studied (Ghodake et al., 2011). A. cepa roots were treated with dispersions of the cobalt and zinc oxide NPs at 5, 10, and $20 \mu \mathrm{g} / \mathrm{mL}$ concentrations. The roots growth inhibited by both the cobalt and the zinc oxide NPs in concentration dependent manner.

The sensitivity of $A$. cepa to cadmium (Cd) stress was investigated. The endpoints screened for genotoxicity included chromosomal aberrations, micronuclei frequency and mitotic frequency. The results indicated that exposure to Cd induced dose-dependent increase in chromosomal aberrations, DNA fragmentation and micronucleus frequency in A.cepa roots (Arya and Mukherjee, 2014).

The CA, MN, MI and NA are attributed to the presence of metals in metallurgical wastes and a number of authors showed these aberrations in other systems also in the presence of metals. For example, $\mathrm{Cu}$ contaminated drinking water was reported to affect the $A$. cepa root merimistematic cell (Fiskesjo, 1981) and $\mathrm{Zn}^{2+}$ and $\mathrm{Cd}^{2+}$ ions were also reported as CA causing agents in the $A$. cepa meristematic root cells (Leme and Marin-Morales, 2009). The metals ions such as $\mathrm{Hg}, \mathrm{Cu}, \mathrm{Ni}, \mathrm{Cd}, \mathrm{Be}, \mathrm{Al}, \mathrm{Mn}, \mathrm{Li}$ induced the CA's like C-metaphases and chromosome adherence in $A$. cepa (Fiskesjo, 1988). Arsenic showed genotoxicity and induced $\mathrm{MN}$ in meristematic as well as in $\mathrm{F}_{1}$ cells of $A$. cepa (Yi et al., 2007a). Water sample containing chromium ions also showed CA and MN formation in A. cepa meristematic cells (Matsumoto et al., 2006) and wastewater contaminated with $\mathrm{Fe}, \mathrm{Pb}, \mathrm{Cr}$ and $\mathrm{Cu}$ showed CA's in A. cepa roots (Abdel Migid et al., 2007). Significant effects on MI and CA and MN induction were also documented when $A$. cepa roots were exposed to Cd metal (Seth et al., 2008b). A considerable CA were also reported in $A$. cepa roots in the presence of $\mathrm{Pb}, \mathrm{Ni}$, $\mathrm{Cr}, \mathrm{Zn}, \mathrm{Cu}$, and $\mathrm{Cd}$ (Rank and Nielsen, 1998). For the detection of genotoxicity of boron, Konuk et al. (Konuk et al., 2007) reported significant induction of CA's in A. cepa meristematic root cells.

\section{Pulp and paper mill}

The pollutants discharged from the pulp and paper industry affect the water bodies adversely (Pokhrel and Viraraghavan, 2004). Change in plankton population has been reported due to untreated paper mill effluent discharge into the system (Baruah and Das, 1997) and (Yen et al., 1996) reported the sub-lethal effects on the aquatic organisms. Another study narrated the effects of treated pulp mill effluents on irrigated soil and revealed the serious adverse soil chemistry changes (Howe and Wagner, 1999). Paddy field was also affected as a result of pulp and paper mill effluents (Dutta, 1999) and high loads of organic pollutants and trace metals in paper mill wastewater (Gupta, 1997; Skipperud et al., 1998). In view of toxic nature of pulp and paper mill wastewater, various authors evaluated the toxicity by $A$. cepa test system.

The $\mathrm{MN}$ and anaphase aberrations were performed by exposing $A$. cepa roots to paper mill wastewater. The acetone extracts of the sludge samples from paper mill were also used for the treatment of $A$. cepa roots and resultant abnormalities were recorded (Grover and Kaur, 1999). In a study, A. cepa root tip cells were analyzed for CA after $24 \mathrm{~h}$ of exposure to paper mill wastewater and results showed considerable effect on CAs and genotoxicity (Nielsen and Rank, 1994). The toxicity of Kraft mill effluent was examined by Tipirdamaz et al. (Tipirdamaz et al., 2003) by A. cepa test system. They treated the $A$. cepa bulbs with wastewater samples and observed the inhibition of root growth (55.72\%). The $A$. cepa also showed a significant induction in cytological abnormalities such as C-mitosis, anaphase with laggard chromosome, fragment and bridges and chromosome stickness in root-tip cells. C-mitosis of $47.7 \%$ was observed in root tip cells treated with paper mill (Kraft) effluent. Taking the total aberrations into account, percentages of laggard chromosomal fragment and bridge formations were $26.6 \%$ and $33.3 \%$, respectively and chromosome stickiness was found to be $12.2 \%$ versus negative control. The banded chromosome was observed at the frequency of $13.3 \%$ of aberrant cells. Chaparro et al. (Chaparro et al., 2010) evaluated the genotoxicity of bleach plant effluents (paper mill) by applying the CA, MN and MI in A. cepa root cells. The largest $\mathrm{CA}$ and $\mathrm{MN}$ incidence in the meristematic cells of A. cepa were recorded after exposure to the untreated bleach plant effluent, however, anaerobic biodegradability reduced the CA's and MN formation. In a study to evaluate the genotoxic effects of paper mill, Gana et al. (Gana et al., 2008) 
revealaed the induction of toxicity in $A$. cepa roots exposed to the tested samples. In another study, the toxicity and genotoxicity tests were performed on root cells of $A$. cepa in order to evaluate wastewater from the bleaching sequence of Kraft cellulose from radiata pine (Pinus radiata). Kraft cellulose from an industrywas specifically obtained from the first extraction stage of the ECF bleaching sequence (Roa et al., 2012). The results revealed a toxic effect of the effluent, with inhibition of meristem growth and generally lower values of metaphase, anaphase and telophase indices at $\mathrm{pH}$ 10.5 than $\mathrm{pH} 7$ for all effluent concentrations. The genotoxicity effect was different from the toxic effect which indicated that the micronucleus and the chromosomal aberration tests in anaphase-telophase cells were low over all ranges of the studied effluent concentrations.

Chaparro and Pires (2011) treated the bleaching effluent from two kraft pulp mills in anaerobic reactor and analyzed for physico-chemical parameters and toxicity $A$. cepa along with other bioassays. Bio-treatment improved the water quality along with cytotoxic, genotoxic and mutagenic effects reductions. Based on results, authors suggested the biotreatment of wastewater before discharge the wastewater and monitoring the treated water using bioassays.

Chaparro et al. (2015) applied an advanced oxidation process (ozone and ozone/UV) to treat kraft cellulose pulp mill and ecotoxicity and genotoxicity were measured before and after treatment with ozone and ozone/UV. The AOX, color and phenol removed up to $40 \%, 79 \%$ and $32 \%$, respectively and $A$. cepa test showed good sensitivity to measure the genotoxicity of treated and un-treated wastewater. In another study, paper and pulp industry effluent treated by Rhodococcus sp. NCIM 2891 were evaluated for cytogentic effects. Along with improvement in water quality parameters (COD, BOD, TDS, heavy metals) toxicity was also reduced considerably (Nadaf and Ghosh, 2014). Results revealed effectiveness of bio-treatment of pulp and paper wastewater and efficiency of $A$. cepa test to evaluate the toxicity difference between treated and untreated effluents.

\section{Textile industry}

The textile industry generates a huge volume of wastewater which contains a varieties of toxic agents such as heavy metals, carboxymethyl cellulose, polyvinyl alcohol, sodium hypochlorite, chlorine, acids, alkali, surfactants, dyestuffs, reducing agents, oxidizing, agents, wetting agents, binders, thickeners and cross-linkers (Adeel et al., 2017; Adeel et al., 2015; Bouatay et al., 2016; Gulzar et al., 2015; Husaain et al., 2015; KANU and Achi, 2011; Mahmood and Malik, 2014; Muneer et al., 2016; Saeed et al., 2016). Researches reported the toxicity of textile wastewater in Daphnia magna (Eremektar et al., 2007), microbil toxicity (Koparal et al., 2007), male reproductive systems of albino rats and mice (Suryavathi et al., 2005). Regarding toxicity evaluation by $A$. cepa, they indentified and studied numerous potentially mutagenic endpoints.
In a study, the CA's, NA and MN were reported due to continuous exposure of $A$. cepa bulb to textile effluent in different concentrations $(0.3 \%, 3 \%, 10 \%$, and $100 \%)$. The mutagenic effect of the effluent at concentrations of $10 \%$ and $100 \%$ were observed. However, at lower concentrations $(0.3 \%$ and $3 \%)$ no abnormities were recorded (Carita and Marin-Morales, 2008). In another study, genotoxic effects of leachates from solid waste of a dye industry have been recorded. The $A$. cepa bulbs were exposed for $48 \mathrm{~h}$ to 2.5$10 \%$ leachates aqeous solution and results revealed the cytogenetic effects in $A$. cepa root. Significant inhibition of MI, CA's and $\mathrm{MN}$ inductions were found in all experimental groups exposed to dye waste leachate aqueous solution (Chandra et al., 2005). Chandra et al. (Chandra et al., 2005) applied the CA test in $A$. cepa to assess the genotoxic effects of dye waste leachate from chromium pigments manufacturering plant. The authors also reported MI inhibition and CA's and MN induction in the roots exposed to leachate extracts.

The genotoxicity of wastewater samples from textile industrial effluent from the Amritsar, India were investigated using the $A$. сера $\mathrm{MN}$ and anaphase aberration assays and effluent exposure to $A$. cepa showed negative responses both in the MN and NA (Grover and Kaur, 1999). In another study, the toxicological analysis of effluents before and after treatment (decolorization and degradation) was performed using $A$. cepa test and results revealed the cytotoxicity and genotoxicity significantly reduced after treatment (Phugare et al., 2011). While studying the toxicity of textile industry wastewater by A. сера, Nielsen and Rank (Nielsen and Rank, 1994) revealed that the wastewater showed a considerable effect on the growth of $A$. cepa roots in five days exposure and CA analyzed after $24 \mathrm{~h}$ of exposure also revealed the CA's effect as compared to negative control. In another study, the cytogenotoxic effects of the textile industrial effluents at different concentration (1.6, 4, 8 and 16\%) were reported by A. cepa assay. The root length, MI and CA's were recorded after $96 \mathrm{~h}$ exposure and results showed that the root growth inhibition was concentration dependent. The MI decreased with increasing concentrations of textile industrial effluents and the CA's such as vagrant chromosome, bridges and fragments and sticky chromosomes were observed in treated group of $A$. сера. At lower concentrations bridges and fragments were the most common aberration (Samuel et al., 2010). Cytotoxicity and genotoxicity studies were performed before and after decolorization of the textile effluent by $A$. cepa root test (Jadhav et al., 2010). Results indicated strong genotoxic effects of the effluents in the mitotic cells before treatment. The control exhibited the MI value of $11.68 \%$, while cells exposed to effluents at a concentration of $30 \%$ showed MI of $9.42 \%$ and further by applying the concentrated solution, the MI decreased significantly. An increase in the incidence of polyploidy cells was also detected and correlated it with effects of the dyes on the -SS and -SH containing proteins of the spindle apparatus. The biotreated samples showed the MI in the range of the control. 
The CA test in $A$. cepa roots was applied to evaluate the genotoxic potential of a silk dyeing industry effluent and response revealed MI inhibition and induction of several mitotic abnormalities in roots cells exposed to the tested effluent (Sudhakar et al., 2001). In order to assess the genotoxic effects of textile effluent Oriaku et al., (Oriaku et al., 2011) investigated the MN, CA's and NA abnormalities in $A$. cepa along with physico-chemical characterization of wastewater. The textile effluent reduced the root length and higher CA's and NA frequencies such as vagrant, sticky chromosomes, binucleus and C-tumors in exposed $A$. cepa root tips as compared to control. The results obtained from this study indicate that textile effluent discharged into the watershed is capable of causing significant ecological disruption in the receiving environment and $A$. сера is a sensitive method for indication disruptions. Few others studies were also performed in order to evaluate the cytotoxic, genotoxic and mutagenic actions of dyes aqueous solution i.e., the toxicity of azo dye at different concentrations $(1,10,100$ and $1000 \mu \mathrm{g} / \mathrm{L})$ was evaluated by $A$. cepa test and it was found that dye induced cell death as well as CA's, NA and $\mathrm{MN}$ even at low concentration (Ventura-Camargo et al., 2011). The CA's in A. cepa roots was correlated with to the presence of azo dyes (CI disperse blue 373, CI disperse violet 93 and CI disperse orange 37) in textile wastewater (Carita and Marin-Morales, 2008).

Balan et al., (2012) treated textile dye malachite green with laccase (white-rot fungus Pleurotus florida NCIM 1243 enzyme) and treatment efficiency was evaluated on the basis of decolorization and detoxification. Under optimized treatment conditions, up to $96 \%$ decolorization of dye was achieved and A. cepa showed toxicity (root growth inhibition) in dye concentration dependent manner. Prasad et al. (2013) used A. cepa test to evaluate the ability of halophilic bacterial strain to degrade the complex azo dye Direct Blue-1 (DB-1). Complete decolorization of DB-1 (100 $\mathrm{mg} / \mathrm{L})$ was achieved in $6 \mathrm{~h}$ at $37{ }^{\circ} \mathrm{C}, \mathrm{pH} 8$ and with $70 \mathrm{~g} / \mathrm{L}$ $\mathrm{NaCl}$. Phytotoxicity study revealed the less toxic nature of the metabolites compared to the parent dye. Genotoxicity test confirmed the toxic nature of DB-1 since several CA's were observed which reduced significantly after treatment. Mansour et al. (2011) examined the performance of Pseudomonas putida mt-2 in treating TE. P. putida was able to completely decolorize the studied effluent after $8 \mathrm{~h}$ of incubation under agitation in an aerobic bioreactor. Genotoxicity of effluent, before and after biodegradation was evaluatedand chromosome aberrations were compared with untreated mice. Results indicated that TE showed a significant ability to induce DNA damage, which was evaluated in both SOS (IF = 3.52) and chromosome aberration assay systems (23.5\%). In addition, TE reduced strongly the acetylcholinesterse (60\%) and butyrylcholinesterase (51\%) activities and induced a remarkable lipid peroxidation effect (increased markedly by approximately fivefold). This toxicity was imputed to the presence of dye compounds of TE. However, toxicity in TE was significantly reduced after $8 \mathrm{~h}$ of aerobic incubation with $P$. putidamt-2 strain. The study demonstrated that $P$. putida mt-2, incubated under aerobic conditions, had a metabolism that enabled it to degrade TE and, especially, to detoxify the effluent mixtures.

The enzymatic (peroxidases from turnip) treated textile dyes toxicity was evaluated using $A$. cepa assay. Biotreated effluents showed less toxicity versus un-treated wastewater (Kulshrestha and Husain, 2007). Similarly, remazol red (RR) was treated with Pseudomonas aeruginosa. Apart from 97\% dye degradation, the dye toxic effect was also reduced (Jadhav et al., 2011). Phugare et al. (2010) treated textile wastewater with yeast cell biomass (waste product from the distillery industry) and $S$. cerevisiae MTCC 463 (SC) and toxicity was evlauted before and after treatment using A. cepa test. Textile effluent was decolorized up to $78 \%$ using SC along with heavy metals removal with both the treatment process. Cytotoxicity, genotoxicity and phytotoxicity studies were performed to evaluate the toxicity of metabolites produced after decolorization of textile effluent, which revaled the efficiency of $A$. серa for detoxification evaluation. Satar and Husain (2011) employed calcium-alginate pectin entrapped bitter gourd peroxidases for the treatment of disperse Brown 1 (DB 1) and Disperse Red 17 (DR 17). Toxicity was evaluated before and after biotreatment. Maximum decolorization of 98\% DR 17 and 71\% DB 1 dyes were achieved at optimized conditions and toxicity tested by A.m cepa test showed good efficiency for the evaluation of bio-treatment effect. Jadhav et al. (2012) treated textile azo dye Remazol orange by bacterium Pseudomonas aeruginosa $\mathrm{BCH}$ in plain distilled water and detoxification was checked by $A$. cepa. Results revealed less toxic nature of the dye after bacterial treatment. Similarly, in another study calcinated coal mining waste contaminated river water and residues derived from coal mines from the Urussanga River (Urussanga, Santa Catarina state, Brazil) were assessed using root growth inhibition test. Untreated water caused significant toxicity, which reduced after treatment (da Silva Francisconi et al., 2013). Iqbal and Bhatti (2014) evaluated the toxicity of un-treated and photocatalytic treated texile wastewater. Cytotoxicity was evaluated through $A$. cepa and heamolytic bioassays. A. cepa test showed that photo-catalytic effluents showed less cytotoxicity and authors revealed that processed wastewater could possibly be used for irrigation purposes in view of significant toxicity reduction. In another study $\mathrm{N}-\mathrm{TiO}_{2}$ nanostructure was used for textile dye treatment and and apart from $97 \%$ degradation, the cytotoxic effect of teated wastewater reduced to a greater extent versus un-treated sample (Kadam et al., 2014). Iqbal et al. (2015) also evaluated detoxification of textile effluents treated by advanced oxidation process. The cytotoxicity of un-treated and treated effluents was measured by root growth. The wastewater irradiated to the absorbed doses of 5kGy, 10kGy, and $15 \mathrm{kGy}$, and subjected to toxicity tests. The gamma radiation showed promising efficiency for detoxification of pollutants in wastewater. The root length and root count increased up to $31.10 \%$ and $38.34 \%$, respectively when roots exposed to wastewater treated to the absorbed dose of $5 \mathrm{kGy}$. 
A. cepa test showed good correlation with heamolytic and brine shrimp test used for cytotoxicity evaluation. Results showed that gamma radiation has promising efficiency for the degradation of toxic agents present in effluents and $A$. cepa proves to be efficient for monitoring the toxicity of treated effluents. Iqbal and Nisar (2015) also treated textile effluents by gamma radiation in combination with hydrogen peroxide and cytotoxicity and mutagenicity was investigated using A. cepa along with shrimp amd Ames tests. Textile effluents were irradiated to the gamma radiation absorbed doses of $5 \mathrm{kGy}, 10 \mathrm{kGy}$ and $15 \mathrm{kGy}$ in combination with $20 \mathrm{mM}$ hydrogen peroxide. Before treatment, textile effluents showed a significant cytotoxic and mutagenic signs, which reduced significantly after treatment. $A$. cepa showed reduction in cytotoxicity upto $50 \%$. Results revealed that gamma radiation in combination with hydrogen peroxide can be implemented for the detoxification of textile effluents and A. cepa assay is excellent in evaluating the toxicity. Machado et al. (2012) also investigated the detoxification of the wastewater produced by the process of rhodamine B dyeing using oxidation processes (Ozonization, ultraviolet irradiation and $\mathrm{O}_{3} / \mathrm{UV}$ ). Cytotoxicity and genotoxicity were assessed of un-treated and treated wastewater using $A$. cepa test along with other assays. Advanced oxidation treatment significantly degraded the rhodamine B. Toxicity results showed that inspite of complete decolorization, dye treated solution was found to be toxic, which might be due to the generation of toxic by-product as a results of destruction of complex dye. While studying the effect of immobilized manganese peroxidase (MnP) on detoxification (Bilal et al., 2016d), A. cepa along with other bioassays was employed. At optimized conditions, up to $87.4 \%$ degradation was achieved along with significant reduction in cytotoxicity i.e., root length, root count and mitotic index increased up to $38.46 \%$, $43.47 \%$ and $41.83 \%$, respectively versus un-treated effluents, which was also confirmed by heamolytic, brine shrimp and Ames tests. Same authors, investigated the efficiency of Ganoderma lucidum IBL-05 MnP immobilized on Sol-Gel matrix and reported that the cytotoxicity was reduced after bio-treatment (Bilal et al., 2016b). Agar-agar gel immobilized MnP treated textile dyes was also monitored for toxicity and $A$. сера test revealed that the toxicity was reduced significantly after bio-treatment (Bilal et al., 2016c). Another study also confirmed the $A$. cepa test efficiency for toxicity monitroring of Ganoderma lucidum IBL-05 crude ligninolytic enzymes (MnP LiP and Laccase) treated textile effluents (Bilal et al., 2016f). Patel et al. (2015) also evaluated the efficiency of $A$. сера to monitor the effectiveness of biotreatment of industrial effluent. Toxicity study of the effluent using $A$. сера revealed that detoxification of the effluent as a result of bio-treatment was significant. The detoxification of textile wastewater using Ganoderma lucidum IBL-05 crude ligninolytic enzymes was also monitored by A. cepa test (Bilal et al., 2016f). A. сера test was applied for cytotoxicity evaluation along with bioassays. The degradation of Masood Textile, Kalash Textile, Khyber Textile and Sitara Textile (Faisalabad, Pakistan) effluents were achieved up to $87.29 \%$,
$80.17 \%, 77.31 \%$ and $69.04 \%$, respectively at optimized conditions. The cytotoxicity tests revealed that biodegradation significantly detoxify the toxic agent in wastewater and author's suggested the use of biodegradation for the remediation of textile effluents along with/ and detoxification monitoring using bioassay to evaluate the bioefficiency of a treatment method.

Balan et al. (2013) investigated the ability of Pleurotus florida (laccase) on coconut flesh as a solid substrate fermentation. The decolorization of two structurally different dyes such as azo (Reactive Blue 198) and triphenylmethane dye (Malachite Green) were analysed and toxicity before and after treatment was evaluated by $A$. cepa test. The decolorization of Reactive blue 198 and Malachite Green at $8 \mathrm{~h}$ was $93 \%$ and $63 \%$, respectiely. A. cepa root inhibition was measured to demonstrate the potential of laccase in the detoxification and bioremediation process and positive response was observed. Jadhav et al. (2015) treated textile effluents by Pseudomonas sp. SUK1 and toxicity was evaluated before and after treatment for comparison. A. cepa test confirmed the severe toxicity of effluent before treatment, which reduced significantly after bacterial treatment. Watharkar and Jadhav (2014) evaluated dyes mixture treated by in vitro grown Petunia grandiflora and Gaillardia grandiflora. Toxicity was evaluated before and after treatment. Metabolites formed after the degradation of dyes revealed the reduced cytogenotoxicity on $A$. cepa roots cells when compared with untreated dye mixture solution. Phytotoxicity study exhibited the less toxic nature of dye mixture after treatment. Watharkar et al. (2013) also treated reactive Navy Blue RX (NBRX) dye by synergistic phytoremediation potential of Petunia grandiflora with its rhizospheric bacterial isolate Bacillus pumilus strain. The biodegradation degraded dyes up to $80.01 \%$ and $76.80 \%$ by $P$. grandiflora and B. pumilus cultures, respectively, whereas consortium decolorized NBRX up to $96.86 \%$. The treated dye exhibited less cytogenotoxicity effect on $A$. cepa roots when compared to NBRX un-treated solution. Similarly, Poonkuzhali et al. (2011) degraded treated azo dye (reactive blue 198) biologically and analyzed for toxicity. $A$. cepa toxicity test indicates that root growth inhibition was dye concentration dependent, which reduced when roots were exposed to treated dyes solution. Similarly, the cytotoxicity and DNA damaging effects of textile effluent at 1 , $2,5,10,25,50$ and 100 (\%) were studied. Roots were exposed for $96 \mathrm{~h}$ and subjected to macro- and micro-analysis (Alimba et al., 2013). Root length inhibition was $50 \%$ and EC50 values were of 16, 35, 6.5 and $8 \%$ for 24, 48, 72 and 96 $\mathrm{h}$, respectively. Decrease in cell proliferation and higher CA's (binucleated cells, sticky chromosomes, chromosome fragments and anaphase bridges) were also observed. These cytogenetic effects were correlated with $\mathrm{Fe}, \mathrm{Cd}, \mathrm{Mn}, \mathrm{Ni}, \mathrm{Cr}$, and other disturbed physic-chemical parameters.

The toxic potential of flyash by $A$. cepa assay and vermicomposting effect on toxicity reduction was studied (Sarojini et al., 2010). Exposed $A$. cepa roots were found coiled and wavy along with MI reduction, however, 
vermicompost did not induce any negative effect on root morphology. The root grown in cow dung, fly ash, cow dung + fly ash and vermicomposted fly ash mixture showed MI values of 9.52, 7.30, 11.43 and 15.76, respectively. Results revealed that fly ash was toxic, which reduced in vermicomposted fly ash and may be beneficial to apply vermicomposted in agriculture.

Recently, the potential toxicity of selected industrial wastewaters reaching the Dandugan Oya in Srilanka, and the downstream water was assessed using $A$. серa. The exposure of $A$. сера bulbs to wastewater and downstream water from the Dandugan Oya resulted in the reduction of root growth $(24-62 \%)$ and mitosis (31 - $55 \%$ ), induction of MN (up to $0.6 \%$ ), nuclear abnormalities (3 - 14 folds) and CA's (3 - 21 folds) in comparison to control. Results revealed that wastewaters under investigation contained cyto-genotoxic agents and authors suggested the use of suitable bioassays as additional assessments in water quality monitoring as indicator of cyto-genotoxic agents in wastewater receiving inland surface waters (Kannangara and Pathiratne, 2015).

\section{Fertilizer and chemical industry waste}

Among chemical fertilizers, the urea and NPK (nitrogenphosphorous-potasium) fertilizers are used most commonly and contributed immensely towards high pollution load in agricultural runoff effluent and ultimately water bodies and soil is contaminated. Typically, a urea plant produced the wastewater has ammonia level of 1381.3 and $1479.7 \mathrm{mg} / \mathrm{L}$ and NPK $2684.5 \mathrm{mg} / \mathrm{L}$. In general the effluent discharged from fertilizer and chemical units may be highly alkaline or acidic having high amount of florides and are responsible for eutrophication, skeletal fluorosis and abnormal calcification of bones (Sridhar et al., 2009).

The genotoxicity tests of fertilizer industry effluent were conducted with $A$. cepa before and after treatment with different kinds of algal biofilters. Root cells were exposed for $24 \mathrm{~h}$ to different dilutions of both raw and treated wastewater and cytogenetic endpoints (CA, MI and NA) were used to assess the potencies of the effluents. Before algal treatment, effluents showed a strong genotoxic effects represented by severe inhibition in mitotic activity of meristematic cells with high frequency of both CA and NA. After algal treatment, the cytotoxic effects of $30 \%$ and $60 \%$ concentrations of the treated effluent were comparable to those of $5 \%$ and $10 \%$ concentrations before treatment, respectively and the frequency of CA's and NA declined by $50 \%$. Statistical analysis of the data indicates a significant reduction in genotoxicity associated with a remarkable reduction in heavy metals concentrations after bioremediation by algal biofilters (Abdel Migid et al., 2007). In another study, $A$. cepa response was studied of the chemical factory effluent in Enugu, Nigeria (Abu and Ezeugwu, 2008). The MI decreased as effluent concentration and treatment durations increased. Significant differences in MI at different concentrations and treatment durations were recorded. Conversely, percentage of abnormal dividing cells significantly increased as the concentration and treatment durations increased. Diverse CA's and cytotoxic problems were observed, ranged from a mild C-mitotic effect at lower concentrations to vacuolated nucleus and even cytokinetic failure at higher concentrations. In another study, the effect of wastewater from a phosphoric gypsum depot on $A$. cepa roots, showed the $\mathrm{CA}$ and mitotic irregularities. In comparison to oak, A. cepa was found to be less tolerant to wastewater from the phosphoric gypsum depot (Pavlica et al., 2000). The chemical industry wastewater induced anaphase-telophase disturbance. A significant difference was observed regarding CA's and root growth inhibition in exposed roots versus negative control (Vujosevic et al., 2001).

\section{Tannery and leather industry}

Wastewater expelled from leather and tannery industries are of great concern and considered to be one of the ten most harmful to the environment, responsible for extreme pollution of water resources and generating substances leading to deterioration and death of a wide range of living organisms (Junior et al., 2007). Various authors reported the toxicity of tannery wastewater against $A$. серa exposed to tannery waste, which led to significant decrease in mitosis and root growth and attributed it to the presence of $\mathrm{Cr}$ and $\mathrm{Ni}$ compounds in it (Chandra et al., 2004). The genotoxicity of leachates of tannery solid waste through aqueous and soil medium has been reported in root meristematic cells of $A$. сера. The experiment was conducted in two sets in which one set of bulbs was placed over aqueous concentration of leachates $(2.5,5 \& 10 \%)$, while in other experiment bulb were exposed to contaminated soil. Root tips, sampled after $48 \mathrm{~h}$ revealed higher frequency of aberrations in aqueous medium as compared to leachate-contaminated soil. However, mitotic abnormalities, CA's and MI inhibition were observed in both experiments (Chandra and Gupta, 2002). In another study, while evaluating the genotoxicity of water contaminated with leather industry wastewater, Junior et al. (Junior et al., 2007) reported evidences of CA's in A. cepa root tip cells. The water impacted by tanneries wastewater, also showed significant frequencies of CA's and MN in exposed $A$. cepa meristematic cells and the abnormalities were correlated with the presence of $\mathrm{Cr}$ ions (Matsumoto et al., 2006).

Matsumoto and Marin-Morales (2004) conducted a study to assess the toxicity and cytotoxicity of water bodies receiving industrial and domestic effluents using $A$. cepa test. To assess the toxicity and mutagenicity of water possibly contaminated with chromium, derived from tannery activities, seasonal water samplings were performed in 2001 and 2002 at five different sites along the Sapucaizinho River, Municipality of Patrocínio Paulista, State of São Paulo, Brazil. A. cepa seeds were germinated in waters from the different collection sites. Results showed that the collection sites most heavily compromised by chromium since low mitotic indices and a higher frequency of mitotic changes such as irregular anaphases (disorganized, multipolar, laggard), cells with 
chromosomal adherences, cells with $\mathrm{MN}$, and binucleate and/or multinucleate cells were observed in exposed roots.

Gupta et al. (2012) studied the cyto genotoxic effects of tannery effluent and chromium (Cr) in A. cepa, from "Up flow Anaerobic Sludge Blanket" (U.A.S.B) Jajmau, Kanpur. A. cepa roots were exposed to various concentrations of tannery effluent $(0.0,3.125,6.25,12.50$ and $25.0 \%)$ and $\mathrm{Cr}$ $(0.0,1.0,2.0,4.0,8.0 \mathrm{mg} / \mathrm{L})$ for 48 and $168 \mathrm{~h}$. Effluent analysis showed pH (8.5), EC (11.94 dSm-1), BOD (499 $\mathrm{mg} / \mathrm{L})$, COD (1382 mg/L) and Cr content (2.32 mg/L), which were much higher than the prescribed permissible limits. A significant reduction in root length, MI and CA's, mitotic aberration and MN were observed. Reduction of 81.15 and $79.71 \%$ in $\mathrm{MI}$, and induction of 6.8 and $4.8 \%$ in CA, 29.24 and $26.66 \%$ in mitotic aberration and 0.52 and $0.43 \%$ in MN were found at $12.50 \%$ of effluent and $4 \mathrm{mg} / \mathrm{L} \mathrm{Cr}$ treated roots versus control.

Poonkuzhali et al. (2013) evaluated the chelating ability of aqueous leaf extract of Aerva lanata. The aqueous leaf extract showed a dose dependent decrease in chelating ability using $\mathrm{FeCl}_{2}$. Aqueous leaf extract removed the chromium from tannery effluent by $43 \mathrm{mg} / \mathrm{g}$. A. cepa toxicity test was performed on tannery effluent treated with aqueous leaf extract that increased the root length significantly, which revealed that Aerva lanata efficienctly removed the chromium from wastewater.

\section{Pharmaceutical, pesticides and herbicides}

Pharmaceutical agent and pesticides are the most common products found in waters. The wastewater discharged from pharmaceutical industries is responsible for microorganisms growth inhibition, reduction in the cell-aqueous phase transfer rates, a sedimentation hindrance due to the development of filamentous microorganisms, development and flotation of sludge with poor activity and clogging (Lateef, 2004).

In order to assess the genotoxicity of pesticides on $A$. cepa, farms contaminated with pesticide and forms where organic forming is used were monitored for toxicity by anaphase aberration and MN induction (Kong and Ma, 1999). Results showed that the soil contaminated with pesticide showed 2.78-3.01 fold higher anaphase aberrations as compared to organic farming soil, while the $\mathrm{MN}$ induction was increased 1.66-4.75 fold in pesticide contaminated soil. Lateef (2007) examined wastewaters from two pharmaceutical production processes, cotrimoxazole B wastewater (BWW) and Piriton wastewater (PWW) for genotoxicity using A. cepa. The effluents induced various types of CA's such as disturbed spindle, vagrant and chromosome bridge, and also showed a dose-dependent reduction in the number of dividing cells. The mitotic inhibition ranged from 38.6 to $67.2 \%$. The mean root length at $20 \%$ of BWW and all concentrations except $1 \%$ of PWW were significantly different from the control. The $\mathrm{EC}_{50}$ of the root growth inhibition was 4.17 and $12.45 \%$ for PWW and BWW, respectively. However, the wastewater physico-

chemical analysis revealed that most parameters were within the permissible limits.

Bakare et al. (2009) investigated the potential genotoxicity of a pharmaceutical industry effluent by A. cepa test along with other assays. The $A$. cepa roots were exposed to different effluent concentrations. The root growth was inhibited. The MI value was also decreased along with CA inductions in a concentration-dependent manner. Results revealed the presence of cytogenotoxic constituents in test samples that may induce adverse health effects in exposed individuals.

Agricultural land receiving pharmaceutical effluents were studied for toxic effects using $A$. cepa on the basis of root growth, MI reduction and aberrant ( $\mathrm{Abu}$ and $\mathrm{Mba}$, 2011). The results revealed a significant reduction in the number and length of roots. The MI reduced up to 52, which was concentration and treatment time dependent. Aberrant cells observed ranged from a C-mitotic effect to anaphase bridges and multiple nuclei induction. In another study, the genotoxicity potential of a pharmaceutical effluent in A. cepa root cells by analyzing the CA's and MN abnormalities were investigated (Bakare et al., 2011). The A. сера assay was carried out in concentrations of $0.5 \%, 1 \%, 2.5 \%, 5 \%$ of the effluent. There was a statistically different, concentrationdependent inhibition of root growth and MI as well as frequest CA inductions. Akintonwa et al (2009) evaluated the mutagenic potentials of effluents from six pharmaceutical companies in the Lagos province, Nigeria using A. cepa assay by measuring root length, MI and CA end points. Onion bulbs were exposed to 5,10 and $15 \%$ concentration of the pharmaceutical effluents. All the six effluents showed mitodepressive and toxic effects to the A. cepa root. In view of toxicity observed, authors recommended that pharmaceutical companies should treat effluents before being discharged into water bodies. Maselli et al. (2015) compared the sensitivities of five ecotoxicity tests using aquatic and terrestrial organisms including $A$. cepa test to evaluate the toxicity of effluents from the production of veterinary medicines before and after treatment. Raw and chemically treated effluent samples were highly toxic. However, toxicity was reduced in biologically treated effluents and bioassays were found to be highly sensitive for toxicity evaluation of raw and treated samples. In another study, cytotoxic effect of the fungicide tilt in root tip cells of $A$. cepa were investigated (Pulate and Tarar, 2014). Roots were exposed to $0.02 \%, 0.04 \%, 0.06 \%, 0.08 \%$ ) concentrations of tilt for $3,6,9,12 \mathrm{~h}$ and as a result of exposure C-metaphase, sticky metaphase, diagonal metaphase, disturbed metaphase, multipolar anaphase, precocious anaphasic chromosome, chromosome bridge at anaphase and laggard were observed.

In order to verify the genotoxic effects of pharmaceutical and pesticides effect, various researchers also evaluated the effect of pesticides and pharmaceutical agents as a model compound (Rank and Nielsen, 1993) i.e., diuron, cypermethrin and fenvalerate (Chauhan et al., 1999; Chauhan et al., 1998), 2,4-D, atrazine \& butaclor (Bolle et al., 2004), isoproturon/diuron and deltamethrin (Chauhan and 
Gupta, 2005a), atrazine commercial formulation (Srivastava and Mishra, 2009), metolcarb (Liman et al., 2010a), commercial formulation of the pyrethroid (Saxena et al., 2005), afugan (Yüzbaşioglu et al., 2003), abamectin \& emaamectin benzoate (Ahmed, 2014), emulsifiable concentrate of cypermethrin, deltamethrin, lambdacyhalothrin \& endosulfan (Yekeen and Adeboye, 2013). The pesticides, herbicides and pharmaceutical were found to induce CA's incuding stickiness, C-mitosis, bridges, lagging chromosomes, fragment, multipolarity, disturbed anaphase-telophase, pro-metaphase disturbance, polyploidy, binuclear cells and disturbed nucleus. The MN formation was also observed along with root growth inhibition, and decreased MI in A. cepa roots exposed to pesticides, herbicides and pharmaceutical agents.

\section{Other industries}

Xing et al. (1995) studied the effect of chemical industrial effluent (from Lanzhou Chemical Industry Corporation) on $A$. cepa root tipcells and it was observed that the mitosis process was disturbed by the industrial effluent. The mitosis of $A$. cepa root tip cell was proved to be very sensitive to environment mutagens, especially to industrial effluent. Therefore, the $A$. cepa test was suggested as a warning of environment monitoring. In another study, electroplating industry wastewater from Madurai city, India was studied for cytogentic effect (Nagarajan et al., 2014). Proportional increase in phtotoxicity percentage with increase in effluents concentration revealed toxicity. Histological abnormalities like bridge formation, fragmentation, multipolarity and stickiness during mitotic cell division in root tip meristamatic cells of $A$. cepa revealed the genotoxic impacts of the effluents. The geno-toxicological effects of olive mill wastewater, olive wet husk and olive brine were compared (Pierantozzi et al., 2012). The roots showed the inhibitory effects on germination and growth. At high concentrations, olive wastes inhibited/suppressed the mitosis process. Authors suggested that direct use of olive wastes for agricultural purposes should be limited owing to their possible genotoxic, chemotoxic and phytotoxic effects. Olorunfemi et al. (2012) studied the toxicity of raw and treated effluents of dairy industry along with physicochemical analysis. The zinc, iron, manganese, nitrates and sulphates were higher than Standards Organisation of Nigeria permissible limits. $A$. cepa roots exposed to wastewater for $96 \mathrm{~h}$ inhibited root growth in concentrationdependent manner. Root exposed for $48 \mathrm{~h}$ to $0.5 \%, 1.0 \%$, $2.5 \%, 5.0 \%$, and $10 \%(\mathrm{v} / \mathrm{v})$ concentrations showed CA's (sticky and laggards chromosomes) at all concentrations, however, toxic effects were reduced in treated wastewater. Authors revealed that combination of physico-chemical analysis and genotoxicity assay is effective in assessing the mutagenic components of industrial effluents for environmental monitoring of pollutants. The treated wastewater from the dairy industry, if discharged into water bodies without further treatment, could pollute the receiving water bodies and impair biolife.
In order to assess the cytogenotoxic effects of paint industry effluents, the $A$. cepa roots were exposed to $7.2 \%, 18 \%, 36 \%$ and $72 \%$ concentrations for a period of $96 \mathrm{~h}$ (Samuel et al., 2010). The exposed roots growth was inhibited, MI and CA's were observed at all concentration tested and at higher concentration, the aberrations were more severe. The CA's observed in root tip cells of $A$. cepa were of vagrant, bridges, fragments and sticky chromosomes types. At lower concentrations only bridges and fragments were observed. While studying the toxic effects of lemon juice factory, sugar factory and mining works wastewater, Gana et al. (Gana et al., 2008) reported positive results of genotoxicity in $A$. серa roots exposed to the tested samples.

Wastewater discharged from brewery industries has high nitrogen, cleaning and washing reagents (Kanu et al., 2006). The cytotoxicity and genotoxicity of brewery, rubber and bottling industries wastewater was evaluated by CA test in A. cepa meristematic cells (Olorunfemi et al., 2011b). After $72 \mathrm{~h}$ of exposure, the cytotoxic (growth retardation and CA's) effects were observed. The root growth inhibition values were recorded to be $35 \%, 50 \%$ and $62 \%$ for bottling, rubber and brewery effluents, respectively. The CA's induced were mostly of sticky chromosomes, bridges, disturbed spindles and fragments. Based on the $\mathrm{EC}_{50}$ values, the bottling wastewater was found most toxic followed by rubber and brewery. Recently, the genotoxic effects of the paint industry effluent $(100 \%, 50 \%, 25 \%, 10 \%, 5 \%, 1 \%, 0.50 \%$ and $0.25 \%)$ (Ikotun, Lagos) was evaluated based on MI and CA endpoints (Njoku et al., 2015). The root of $A$. cepa exposed to the various concentrations of the effluents showed significant difference in root lengths. There was a significant difference in phase index and mitotic inhibition and decrease MI values were recorded in concentration dependent manner. The effluent induced CA's in the meristematic cells of $A$. cepa root tips and laggards were the most frequently observed aberrations. Akinsemolu et al. (2015) evaluated the effect of industrial effluent from tobacco industry. Healthy sprouted onion roots were treated with concentrations of $20 \%, 50 \%$ and $100 \%$ for 6 to $18 \mathrm{~h}$. The MI value changed significantly at 6 and $12 \mathrm{~h}$ exposure time. However, exposure of root cells beyond 12 led to a complete inhibition of mitosis division even at lowest concentration,. Moreover, the treatment concentrations were also reported to induce CA aberrations like stickiness, C-metaphase, bridges, unequal distribution of chromosomes, breaks, laggards, vagrant and ring chromosomes at concentrations of $20 \%, 50 \%$ and $100 \%$ in 6 and $12 \mathrm{~h}$ exposed roots. Observations revealed that tobacco wastewater had genotoxic potential and capable of damaging DNA, wheras A. cepa proved to be very sensitive to the toxicity for each concentration and exposure time. Mishra (1993) monitored the cytotoxic effects of distillary waste from a sugarcane factory (Kanpur, India) by measuring CA's. Exposure roots revealed that the distillary waste induced damage i.e., the mitosis was disturbed even at $1.5 \%$ and $2.0 \%$ concentration of effluents.

Raw and vermicomposted (Eisenia fetida + cattle dung) sugar beet mud and pulp (waste by-products of the 
sugar industry) waste toxicity was monitored (Bhat et al., 2015). A. cepa root exposed to raw and vermicomposted were analysed for CA's abberations. Vermicomposted sugar beet mud waste reduced the toxicity in the range of $18-75 \%$. Firbas and Amon (2013) tested the communal waste water cleaning plant of the type LIMNOWET® Constructed Wetlands for toxicity by measuring aberrations of the metaphasic chromosomes of the $A$. cepa exposed roots. However, constructed wetlands plant reduced the degree of genotoxicity in the range of $3.5 \%$ to $29.0 \%$. Bhat et al. (2014) investigated the genotoxicity of pressmud (sugar cane industry waste), which was mixed with cow dung at different ratios and vermicomposted using Eisenia fetida. Different concentrations of $100 \%$ pressmud sludge extract $(10 \%, 20 \%$, $40 \%, 60 \%, 80 \%$ and $100 \%$ ) were analyzed for CA's before and after vermicomposting. Percent aberration was highest $(30.8 \%)$ after exposure to $100 \%$ pressmud extract after $6 \mathrm{~h}$ expure, which reduced to $20.3 \%$ after vermicomposting. Among CA's, c-mitosis, delayed anaphase, laggards, stickiness and vagrant aberrations were observed along with significant inhibition of MI. However, after vermicomposting the MI value was increased. Author's revealed that vermicomposting could be an important tool to reduce the toxicity of pressmud as evidenced by the results of genotoxicity evaluated by $A$. cepa test. Viana et al. (2014) evaluated the cytotoxicity and the mutagenicity of liquid waste produced in the process of industrialization of bitter cassava (olho-junto variety). The liquid wastes werwe characterized as press water, which is obtained when the cassava roots were pressed; pond water, which was press water stored in impounded ponds; and a solution of sodium thiosulfate, pure and with other waste. Treatment with saline solution was cytotoxic for $A$. cepa, which reduced the cell division. The thiosulfate solution was clastogenic and induced CA's. Based on results of toxicity assesd by $A$. серa, authors suggested prohibition of sodium thiosulfateuse. Masood and Malik (2013) evaluated the genotoxicity of industrial wastewaters from Jajmau (Kanpur) using $A$. сера anaphase-telophase test. Amberlite resins concentrated wastewater was found to be more mutagenic as compared to those of liquid-liquid extracts (hexane and dichloromethane extracts). The MI values were decreased significantly when roots were exposed to $5,10,25,50$, and $100 \%(\mathrm{v} / \mathrm{v})$ wastewater concentration. Complementary to the lower levels of MI, the wastewaters showed higher CA's in all cases investigated. In another study, the cytotoxic and genotoxic effects and the anatomical changes caused by the effluents arising from the ornamental rock polishing industry (Nova Venécia, State of Espírito Santo, Brazil) were assessed using $A$. cepa assay. The roots were exposed to the effluent at $12.5,25,37.5,50,75$, and $100 \%$ concentrations for 20 days. MI value decreased in concentration dependent manner along with CA inductions and NA's. A high frequency of cells death was observed in the roots exposed to the higher concentrations. Similarly, the toxicity of wastewater and physico-chemical analysis was peformed using A. capa (Olorunfemi et al., 2015a). A bilge water (1, 5, 10, 25 and $50 \%$ ) showed DNA polymorphism which was reflected by changes in the RAPD profiles as variation in band intensity, disappearance of bands and appearance of new bands, was induced by the different concentrations of bilge water on the genome of Allium cepa root cells. The genetic distances shown on the dendrogram revealed that the genotoxicity of the wastewater was concentration-dependent. This study has shown that polymorphism detected by RAPD can be considered as a powerful molecular marker assay for the detection of the genotoxic effect of bilge water. These studies revealed that the wastewater samples from most of the industires induced cytogentic effects, however, A. cepa assay found to be highly sensitive to monitor the toxicity of raw and treated efflunets at various concentration and all researchers suggested the toxicity monitoring of efflunets to avoid contamination of water bodies with toxic agents.

\section{Contaminated river water/surface water/sea water}

The contamination on account of industrial growth directly affects not only the soil and water systems in industrial areas, but also agricultural fields as well as the beds of rivers and canals. Among different types of pollutants in the soil system, heavy metals are of great concern since heavy metals $(\mathrm{Cr}, \mathrm{Cu}$, $\mathrm{Ni}, \mathrm{As}, \mathrm{Hg}, \mathrm{Pb}$ and $\mathrm{Zn}$ ) are well known as carcinogens and deposited in soils and are mobilized either by leaching or by uptake through plants (Katnoria et al., 2011).

The contamination of soil and water sheds and their effects on living systems have been documented in various studies and a number of studied highlighted the genotoxic effects of soil, contaminated with industrial wastewater i.e., the genotoxicity of contaminated soil with industrial wastewater near Metz, France using A. серa MN assay was evaluated. Results showed formation of MN in treated $A$. cepa roots (Cotelle et al., 1999). Similarly, CA, MN, MI and cell death in $A$. cepa root tip cells have been recorded, exposed to river water contaminated with industrial effluents (Bianchi et al., 2011). In another study, the Todos os Santos River (TSR) in Brazil which receives a high discharge of untreated effluents was tested for toxicity (Blanc et al., 2013). The samples were collected at different points (two upstream of an urban area, two in the urban area, and two downstream of the urban area). The concentrations of Al (21.63-1688.84 $\mu \mathrm{g} / \mathrm{L}), \mathrm{P}(38.59-1760.87 \mu \mathrm{g} / \mathrm{L})$, and Fe (478.9-8296.3 $\mu \mathrm{g} / \mathrm{L})$ were beyond the permissible limits. Based on the $A$. cepa test, all river water samples induced genotoxic and mutagenic effects. Simialrly, sediment elutriates from Tambis River, Barobo, Surigao Del Sur, Philippines were invetigated for possible cytotoxicity in $A$. cepa roots (Fajardo et al., 2015). Result showed that at higher concentrations $(1000 \mathrm{~g} / \mathrm{L}$ and $500 \mathrm{~g} / \mathrm{L}$, for $72 \mathrm{~h}$ expoure) exerted mitodepressive effect, Cmitoses, spindle damage and CA's (sticky metaphases and anaphase bridges). Results confirmed the cytotoxic and mutagenic effects of sediment elutriate from Tambis River and $A$. cepa test was found to be very sensitive in detecting toxicity.

The toxicity and mutagenicity resulted from sodium metabisulfite in sea waters and sediments collected in a shrimp farm in Brazil was investigated(da Costa Machado 
Matos Carvalho et al., 2011). A. cepa roots were exposed towater and sediment at concentration of 25,50 , and $10 \%$ for $72 \mathrm{~h}$ (collected in the dry and in the rainy seasons). At the end of $72 \mathrm{~h}$ exposure, $A$. cepa roots revealed considerable negative effects on MI, CA's and MN. The Taquari river water (Brazil) also showed cytotoxicity against $A$. cepa root meristematic cells such as MI inhibition and chromosomal abnormalities (da Costa et al., 2011). In another study, the $A$. cepa root test was used to evaluate the genotoxicity of the Pitimbu river water, Natal (Brazil). The water was collected from five sites along the river and one sample was obtained after the treatment (flocculation, chlorination and $\mathrm{pH}$ correction). All raw river water samples increased the frequency of CA's and MN and two of the water samples affected the MI value. Two of the water samples also altered root growth and two produced morphological modifications in the $A$. cepa roots. Water collected from a site near an industrial area was found to be most toxic (Egito et al., 2007).

Water reservoir from Vazante-MG city (Brazil) (zinc producer district) was tested for toxicity and all collected samples revealed higher concentration of heavy metals and genotoxic signs in $A$. cepa exposed roots, which revealed contamination of well-water in the city since genotoxity was correlated well with heavy metal load detected in water samples (da Silva et al., 2013). Paiva et al. (2008) examined the São Gonçalo Channel (water supply source of the city of Pelotas, Brazil and the surrounding region) for mutagenic activity. Water samples were collected from four different stations (Lock Dam, Santa Bárbara Channel, Pelotas Creek, and Barra do Laranjal). The channel water showed a significantly greater number of abnormalities (MI, mitotic anomalies, interphase anomalies and total anomalies) and in comaprison to previous years, toxic substances in the channel were considerable higher. Orathupalayam Dam water (India) was tested for physico-chemical parameters and toxicity. A. cepa roots grown in the Orathupalayam dam water sample revealed a cytological damage (Gajalakshmi and Ruban, 2014). de Oliveira Meneguetti et al. (2012) studied mutagenicity in river Vale do Jamari. The MI value reduced as well as $\mathrm{MN}$ and CA induction (anaphase and telophase bridges) were frequent in roots exposed to contaminated river water. Author recommended application of $A$. cepa test for monitoring of river water. Júnior et al. (2007) also studied the genotoxicity and toxicity of water and sediment samples collected from the Estância Velha stream of southern Brazil (a stream transporting both domestic sewage and effluents from regional factories working in the leather industry). Samples were collected from stream headwaters (Site 1), downstream of an urban area (Site 2), and near the basin outfall (Site 3). A. cepa results showed no evidence of chromosomal mutation, either in water or in sediment, during winter or summer seasons, but samples collected below Site 1 showed high toxicity. Results suggested that the synergy among different compounds in domestic and industrial sewage discharges could make it difficult to maintain system stability. Similarly, Vujošević et al. (2008) evaluated water samples from river Rasina (Serbia) for potential toxic and genotoxic effects using the $A$. cepa anaphase-telophase test. Inhibition of root growth was observed. Anaphase and telophase analysis revealed that seven among nine samples were highly toxic. Changes in the relation between spindle and chromosome types of aberrations were found in few samples, indicating differences in the potential genotoxic substances present at the analyzed sites. The samples collected at the highest level of river water indicated that the river water might be more toxic during periods of low flow. So, A. cepa anaphasetelophase test was recommended as a monitoring system, which can serve as an alert of contamination of system with toxic agents.

In order to evaluate the toxicity of river water contaminated with industrial effluents in areas of Tucuman (Argentina) CA and MN tests were applied (Gana et al., 2008). A significant aberrant anaphase and $\mathrm{MN}$ inductions were observed. In another study, while studying the toxicity of river water from the Upper Silesia Coal Basin (Poland) Geras'kin et al., reported clear genotoxic effect such as chromatid (single) fragments and bridges, chromosome (double) fragments and bridges, lagging chromosomes and multipolarity in A. cepa root meristem cells (Geras'kin et al., 2011). Genotoxicity and toxicity by $A$. cepa test of Sinos river, Rio Grande do Sul State (Brazil), receiving wastewater from industries (shoes, leather, petrochemical and metallurgy) and domestic wastewater was evaluated (Nunes et al., 2011) and all water samples showed cytotoxic, genotoxic and mutagenic effects. In another study, the microscopic and macroscopic effects in A. cepa meristematic cells, exposed to water from the Drava river, Maribor have been observed. The $A$. ceap roots were exposed to raw water and biologically treated plant output water. The raw water caused sub-lethal and lethal effects and the root length and MI were decreased up to $74 \%$ and $66 \%$, respectively and 10 time more aberrant cells were observed. The $A$. cepa roots exposed to biologically treated water showed only 3\% reduction in MI (Smaka-Kincl et al., 1996).

Fawole et al. (2008) analysed pollution load in water collected from Alamuyo River in Ibadan (Nigeria) and surrounding wells. Water sample analysis and $A$. cepa results revealed toxic substances in all the water samples. High microbial load was observed in river water samples compared to the well water samples throughout the sampling periods with overall highest value observed at late rainy season upstream water sample. Microscopic evaluation of $A$. cepa cells showed decreasing number of dividing cells and MI values, however, this behavior was not dose dependent. Chromosome fragments, bridge, lag and disturbed spindle were the aberrations observed in the study. The results showed presence of toxic substances and high microbial load in river water samples which have also been infiltrated into the surrounding wells. Using MN and anaphase analyses and mitotic indices in A. ceap Ivanova et al. (2005) studied the river water from the region of Panagjurishte, Southwest Bulgaria contaminated with heavy metal and cyanide 
(Ivanova et al., 2005). The data obtained showed decreased cell reproduction and the presence of deviations from the normal mitosis in the form of $\mathrm{MN}$, anaphase and telophase bridges and fragments, lagging chromosomes and C-mitosis. The cytogenetic analysis was found very effective and suitable for biomonitoring of heavy metal and cyanide contaminated water.

The genotoxicity potential of contaminated Taquari river (Brazil) was studied on the basis of CA's induction in $A$. сера. The $A$. cepa test showed significant cytotoxicity and mutagenic index (da Costa et al., 2012b). From same country (Junior et al., 2007), reported the genotoxicity of water from Estancia Velha stream under the effect of domestic sewage and leather industry effluents. Kopliku et al. (2012) used A. cepa grown in water samples from Shiroka, Zogaj, Shegan, Kamicë, Stërbeq, Buna bridge, Bahçellek, Zues and Dajç to evaluate the cytotoxic and genotoxic effects, by measuring root length, MI, Phase Index and CA. The root length decreased in the order tap water, Stërbeq, Kamicë, Shiroka, Buna bridge, Zogaj, Bahçellek, Dajç, Zues, Shegan. The root length and MI values of Dajç, Zues and Shegan were compared to tap water. Most frequent CA types were: stickiness, bridges and fragments. CA formation was in following increasing order tap water, Kamicë, Stërbeq, Buna Bridge, Shiroka, Dajç, Bahçellek, Zogaj, Shegan and Zues. The number of abnormal dividing cells was significant at Dajç, Bahçellek, Zogaj, Shegan and Zues. The CA higher rate was observed at Zogaj. The results indicated slight water pollution in Dajç, Shegan, Bahçellek, Zogaj and Zues samples, serving as a first alert of chemical pollution environmental impact, even at low concentrations.

While studying the Atibaia river toxicity under the influence of petroleum refinery (Hoshina and Marin-Morales, 2009), MN and CA tests were applied. A. cepa were germinated in waters collected from five different sites and also in treated water. Results revealed presence of CA's and MN induction and treated samples (physicochemical, biological and stabilization pond) also induced the CA's and MN abnormalities in meristematic cells of $A$. cepa, however, the effect was lower in comparison to untreated sample and authors suggested an alternative treatment method for wastewater treatment before being discharged in to water bodies. In another study (Smaka-Kincl et al., 1996) A. серa bulbs were grown in undiluted industrial and municipal waste water; biological treated and water from the Drava river upstream and downstream of the city of Maribor, Slocenia and effects were evaluated on the basis of aberrant cells in metaphase and anaphase, index of MN appearance and inhibition of cell division. The untreated industrial and municipal waste water caused lethal and sub lethal effects and the inhibition of root growth, decrease of mitotic index, interphase cells with micronuclei and aberrant cells were found $74 \%, 66 \%, 3 \%$ and $10 \%$ in comparison to control test.

While studying the cytotoxic and genotoxic effect of heavy metal and cyanide contamination (Ivanova et al., 2008) reported the MI inhibition, CA and MN effects in $A$. cepa. Among CA's, chromosome fragments, anaphase and telophase bridges, lagging chromosomes, C-mitosis were the major abnormalities and these abnormalities were correlated with the presence of $\mathrm{Cu}, \mathrm{As}, \mathrm{Cd}, \mathrm{Pb}$ and cyanides. Similar to this study (Staykova et al., 2005) also reported the cytogenetic effect of heavy metals and cyanide polluted waters from the region of Panagjurishte, Southwest Bulgaria by $A$. cepa test. A decreased cell division rate and deviation from the normal mitosis was observed along with chromosomal mutations. In order to assess Danube river water toxicity contaminated with petrochemical complex, oil refinery and chemical industry effluents (Vujosevic et al., 2001) reported the anaphase-telophase aberrations in $A$. cepa cells even after five months of contamination. Clear inhibition of growth and CA were seen in sample, taken just after entry of the canal in to river, while upstream sampling showed no effect. In another study, the CA's and MN in $A$. cepa roots in Belgrade, Yugoslavia were linked to hydrocarbons and polycyclic aromatic hydrocarbons found in river water. The larger CA's and MN incidence in the meristematic cells of $A$. cepa was observed after exposure to water sample collected during the dry season, where the oil leak has arisen (Leme and Marin-Morales, 2008). (Barberio et al., 2009) studied the cytotoxic and genotoxic potential of water from the River Paraíba do Sul (Brazil) and found the inhibition of root length, MI and MN induction. Similarly, (Geras'kin et al., 2011) evaluated the genotoxic potential of water samples from the coal mining area, Poland and clear genotoxic effects of contaminated water were observed in $A$. cepa root cells with severe cytogenetic abnormalities. de Lima Moraes and Jordao (2001) evaluated the cytogenotoxic effects of wastewater from Corumbá and Paraguay River water in flood and drought seasons. A. cepa root meristems were exposed to 20 and $72 \mathrm{~h}$ water and MI, frequency, and aberrant cells types were measured. The wastewater was genotoxic and flood and drought influenced the levels of genotoxicity in river water. Siddiqui et al (2011) germinated A. сера seeds in industrial waste water and river water (India) to measure toxicity/genotoxicity. A. cepa responded significantly with the test range of heavy metals and phenolics. The toxicity of heavy metals was in the order of $\mathrm{Cu}$ $>\mathrm{Ni}>\mathrm{Cd}$, whereas 2,4-dinitrophenol was the most toxic among the phenolic compounds. Among CA's, bridges, stickiness, and fragmentations were recorded with both the industrial wastewater and river water samples in $A$. cepa roots. Akinboro et al. (2011) collected four water samples immediately and five days after rainfall from two locations form Sungai Dua River and tested for toxicity using the $A$. сера assay. The water samples were found to contain sodium and calcium and exposed roots showed inhibitions in root growth and MI. However, the inhibitory effects were not dose-dependent. The results showed the presence of agents in water samples of Sungai Dua River that have caused mitodepressive and genotoxic effects.

The toxicity and genotoxicity of Vistula river water (Poland) from selected points in Warsaw (downstream from Gruba KaĞka) using $A$. cepa test was also studied (OBIDOSKA and LICHMIRA, 2013). The city water-intake 
facility 1-GK (Gruba KaĞka) was nontoxic, however, downstream toxicity and genotoxicity increased until 3-MP (Most Poniatowskiego). The highest toxicity and genotoxicity was observed at point 5-KB (Kolektor Burakowski), receiving untreated municipal sewage directly into the Vistula and below point 6-OC (Czajka Treatment Plant) no toxicity was observed. Düsman et al. (2014) monitored the cytotoxic potential of water of Quatorze River, Francisco Beltrão, Paraná, Brazil, along its route in the rural area, using the root meristematic cells of $A$. серa. The results showed that the water at points 2,3 , and 4 were not cytotoxic because the rates of $A$. cepa cell division were unaltered and at point 1 , MI value was decreased, indicating that this water contained substances with mitogenic capacity. However, the MI values decreased along the route of the river (point 1 to point 4), possibly indicating a mechanism of self-purification, despite having received other sources of pollution. Thus, from results of this study it was concluded that the water of the Quatorze River should be monitored to evaluate contamination and to maintain the quality of the river water. Lindqvist (2015) investigated contamination of river Vallkärrabäcken, Sweden 34 years after the closure of the landfill, Sankt Hans. The $A$. cepa bioassay was used to analyze the water quality in this study, including root growth inhibition and other macroscopic effects. Water samples were taken $0.05 \mathrm{~km}$ away from Vallkärrabäcken to the Sankt Hans landfill as well as $0.5 \mathrm{~km}$ and $2 \mathrm{~km}$ downstream. A. cepa bulbs were grown in Styrofoam frames which floated giving $A$. cepa constant contact to the samples of the river water for 4 days. The $A$. cepa roots growth inhibited along with fewer roots mass and number in water sample taken $0.05 \mathrm{~km}$ downstream. At 0.5 $\mathrm{km}$ downstream the landfill, the bulbs were significantly inhibited in mass of roots and number of roots. At $2 \mathrm{~km}$ downstream the landfill, the bulbs were only significantly inhibited in number of roots. Mazzeo and Marin-Morales (2015) exposed A. cepa seeds to water samples from a river that receives untreated urban effluent and to the trifluralin herbicide $(0.84 \mathrm{mg} / \mathrm{L}$ concentration $)$, both analyzed in two different seasons (summer and winter seasons). Samples induced significant frequencies of $\mathrm{CA}, \mathrm{NA}$ and $\mathrm{MN}$. The herbicide caused a significant increase in the number of nucleoli and MN. Tabet et al. (2015) examined the mutagenicity and genotoxicity of urban wastewater, Guelma city, Algeria between April 2012 and April 2013 using A. cepa $\mathrm{CA}$ and MI end points. The samples were collected from five different sampling stages (S1-S5). A significant decrease in MI was observed with a decrease in the percentage of cells in the prophase and an increase in the telophase. Among CA's, anaphase bridges, disturbed anaphase-telophase cells, vagrants and stickiness in anaphase-telophase cells were observed. All treatments of wastewater in April 2012, at S5 in July 2012, at S1 and S5 in November 2012, at S5 in February 2013, and at S1 in April 2013 induced CA's. Athanásio et al. (2014) investigated the genotoxic, mutagenic, and cytotoxic potentials of surface waters in urban streams using $A$. серa test. Water samples were collected from three streams located in the urban area of a municipality in the south of
Brazil. The frequent MN and CA induction and decrease in the MI value indicate the cytotoxicity and mutagenicity naure of collected samples. The Pedras stream receives mixed industrial and urban wastewater, while the Lajeado and Preto streams receive wastewater predominantly domestic in nature, and A. cepa shown to be extremely sensitive in detecting the genotoxicity of environmental water samples because significant difference was observed among samples tested and this test can be applied as the first tool for environmental health hazard identification and prediction. Roy et al. (2015) studied the toxic effects of tannery effluent (TE) from a town (Ambur), located on the banks of Palar River, India. The phytotoxic effect of TE was tested on A. cepa roots. Exposed roots revealed inhibition of growth. Results revealed that the Palar River of Ambur has been contaminated with agents, which can exert adverse effects on living organisms even at very low concentrations.

Barbério et al. (2009) investigated the cytotoxic and genotoxic potentials of water, River Paraíba do Sul (Brazil) by A. cepa assay using root growth, mitotic indices and MN end points. A cepa roots were exposed for 24 to $120 \mathrm{~h}$ to wastewater collected in the years of 2005 and 2006 from sites in the cities of Tremembé and Aparecida (São Paulo state, Brazil). The roots treated with samples of the river water collected in 2005 decreased the root length. However, MI values were reduced for samples collected from both sites. Considering the data involving root length growth and MI values, a cytotoxic potential was suggested for the water of River Paraíba do Sul at Tremembé and Aparecida. Espinoza-Quiñones et al. (2009) studied $\mathrm{Cr}(\mathrm{VI})$ contaminated river water using $A$. cepa root assay and clean river water caused a $50 \%$ reduction in root length with $4.2 \mathrm{mg} / \mathrm{L} \mathrm{EC}_{50}$ value, while in organically polluted samples similar root growth inhibition occurred at $12.0 \mathrm{mg} / \mathrm{L}$. The results suggested that there was a dislocation to higher values in toxic chromium concentration in polluted river water due to the eutrophization level of river water. Matos et al. (2011) evaluated the toxicity and mutagenicity caused by sodium metabisulfite in sea waters and sediments collected in a shrimp farm in Cajueiro da Praia (Luis Correia), state of Piauí, Brazil, using the A. cepa assay. Water and sediment samples were collected in the dry and in the rainy seasons from three sites: upstream the shrimp farm (Site 1), at the point where sodium metabisulfite is discharged (Site 2), and $100 \mathrm{~m}$ downstream the farm (Site 3). Roots were exposed to 50\%, $25 \%$ and $10 \%$ dilutions for 72 -h and then subjected to MI, CA and $\mathrm{MN}$ analysis. The A. cepa assay revealed that the water and sediments samples collected in the Piauí coast contaminated with sodium metabisulfite induced toxicity and authors revealed that assay may be used as a regular tool in the analysis of water in shrimp farms in the coast of Piauí state for preserving the region's marine ecosystem. More recently, Salles et al. (2016) collected water samples from Jaguari River and Ribeirão Lavapés, Brazil in dry season (August 2011 and 2012) and rainy season (February 2012 and 2013) and evaluated for toxic effects. MN, MI and mitotic anomalies were measured in exposed roots. The stickiness, c- 
mitosis, multipolarity, chromosome bridges were detected in roots exposed to sample collected in rainy season $(8.61 \pm 3.65)$ and dry season $(7.07 \pm 2.96)$. The MN formation and less MI value were higher in the February 2012 samples than in the August 2012 samples. The aberrations were positively correlated with manganese concentration $(0.13 \mathrm{mg} / \mathrm{L})$, which was higher than permitted concentration of $<0.1 \mathrm{mg} / \mathrm{L}$. Overall, samples collected in rainy season were more toxic than the dry season samples.

\section{Wastes contaminated soil}

Meier et al. (1997) employed $A$. cepa test to evaluate the treated soil from a site heavily contaminated with polychlorinated biphenyls (PCBs). Although the PCB content of the soil was reduced by $99 \%$ (after remediation), however, the phytotoxicity and genotoxicity were higher for the remediated soil compared to the untreated soil. The toxicity remaining after treatment appeared to be due to residual solvent introduced during the remediation process, and/or to heavy metals or other inorganic contaminants not removed by the treatment. Results demonstrated the need for including toxicity measurements in the evaluation of technologies used in hazardous waste site remediations for determining the efficiency of remediation processes. While monitoring the toxicity of mercury in aquatic and terrestrial environments, Dash et al. (1998) applied $A$. cepa MN assay. Four mercurial derivatives, namely mercuric chloride (MC), methyl mercuric chloride (MMC), phenyl mercuric acetate (PMA) and a methoxy ethyl mercuric chloride based fungicide, Emisan- 6 were tested to assess the sensitivity and versatility of the $A$. сера $\mathrm{MN}$ assay. $A$. серa bulbs were set directly on water and soil contaminated with known levels of mercurial derivatives (0.0001-10.00 ppm) for five days and then, measured the root length, mitoses with spindle abnormality and $\mathrm{MN}$ inductions in root meristems. The effective concentrations of the test chemicals that caused $50 \%$ of root length as compared to control $\left(\mathrm{EC}_{50}\right)$ were determined from dose-response curves so obtained. The lowest effective concentration tested (LECT) and highest ineffective concentration tested (HICT) for each of the mercurial derivatives for the induction of spindle malfunction and $\mathrm{MN}$ were determined. It was found that $\mathrm{EC}_{50}$, LECT and HICT values for mercurial derivatives in soil were higher than those in water. The frequencies of cells with MN and mitosis with spindle abnormality were highly correlated indicating that $\mathrm{MN}$ is a good parameter of spindle malfunction. The approach applied confirmed the sensitivity of the $A$. cepa assay by 10 -fold, the detection limit being 0.001-0.1 ppm and 0.1-1.0 ppm in aquatic and terrestrial environments respectively, depending on the species of mercury.

The general toxicity (root growth inhibition and malformation) and genotoxicity (induction of chromosome aberrations in root cells) of an oil field wastewater have been investigated by the Allium test. A series of 10 small bulbs of Allium cepa L. were cultivated in various concentrations of the wastewater, and after $48 \mathrm{~h}$ one root tip from each bulb was harvested and processed for cytological studies by the aceto-orcein squash technique. After $96 \mathrm{~h}$, mean lengths of root bundles were obtained and the Effective Concentration (EC) values were calculated. Treatment with wastewater resulted in significant dose-dependent root growth inhibition. $\mathrm{EC}_{50}(96 \mathrm{~h})$ was $28.5 \%$ while a total phytotoxic effect was induced by the undiluted sample. The wastewater is mitodepressive and increased significantly the frequency of chromosome aberrations in root cells (sticky chromosomes, c-mitosis, spindle multipolarity, bridges and fragments). At lower concentrations c-mitosis was the most common aberration. The suitability of the Allium test in genotoxicity screening was highlighted and the impact and significance of positive results on the environment and human health were discussed. Saxena et al. (2004) evaluated toxicity of soil contaminated with Diuron (herbicide) using root meristem cells of $A$. сера. Diuron 22.5, 45.0, and 90.0 ppm were mixed in soil and roots were exposed to soil for 48 $\mathrm{h}$ and analysed for mitotic/CA's. Dose-dependent inductions of mitotic as well as chromosomal breaks were observed. More frequency of mitotic aberrations was observed than CA's. Mild percentage of MN and binucleated cells were observed. Results revealed the possible interaction of diuron with DNA. From Saharanpur and Aligarh, India, Tabrez and Ahmad (Tabrez and Ahmad, 2011) reported the toxicity of soil contaminated with industrial effluents tested using $A$. cepa test. The water sample from two territories increased the frequency of $\mathrm{CA}, \mathrm{MN}$ along with MI reduction. Among CA's, bridges and fragmentation of chromosome were the predominant effects of the Saharanpur water sample, while the Aligarh sample induced mainly fragments.

In order to assess the genotoxicity potential of soil from agricultural fields (irrigated with industrial wastewater) from Amritsar, India, Katnoria et al. (Katnoria et al., 2008) employed $A$. cepa root anaphase aberration assay. The genotoxic potential of soil samples was correlated with content of heavy metals like $\mathrm{Cr}, \mathrm{Co}, \mathrm{Cu}, \mathrm{Mn}, \mathrm{Hg}, \mathrm{Ni}$ and $\mathrm{Zn}$. It was observed that soil samples, which showed the highest percentage of aberrations, also contained higher amount of one or more metals studied. Same authors later, (Katnoria et al., 2011) employed the $A$. серa anaphase aberration assay in order to assess the genotoxicity and mutagenicity of soil samples contaminated with Zn coating industry and copper sulphate manufacturing industry wastewater. The anaphase aberrations ranged from $3.63-10.67 \%$ and $0.38-4.83 \%$ for soil samples contaminated with zinc coating industry and copper sulphate manufacturing industry wastewater, respectively. In another study (Crebelli et al., 2005) showed MN induction and MI inhibition in A. cepa roots exposed to industrial waste contaminated soil near Metz, France. Monarca et al. (Monarca et al., 2000) reported the genotoxicity of contaminanted soil with industrial wastewater using $A$. cepa from industrialized area in the Lombardy region, in Northern Italy and treated group of plant showed strong effect on MN formation and soils sample showed high concentrations of PAHs and heavy metals. Chahal et al. (Chahal et al., 2012) reported the genotoxicity of 
pesticides, inorganic fertilizers and industrial effluents contaminated soil from Nangli village of Amritsar, Punjab, India by employing $A$. cepa root CA's. The treatments resulted in different types of CA's like lagging, vagrant, C-mitosis, delayed anaphase, stickiness, chromosomal breaks and chromatin bridges (clastogenic aberrations) and 12.81\% aberrant cells were found in treated sample as compared to negative control. de Souza Pohren et al. (2013) evaluated solubilized soils (three points) contaminated by heavy metals from municipality of Triunfo, state of Rio Grande do Sul, Brazil considering germination index, MI, CA and mutagenicity index by $A$. сера. Significant responses of CA were observed in the two samples of contaminated soil, but mutagenicity was significant only for soil 3. A. cepa test system was sensitive to investigate the genotoxicity of the soil samples and can be used as an alert in studies on soil contamination.

Kataeva et al. (2012) used A. cepa root MN bioassay to determine the toxicity of 5 soil samples i.e., coppersmelters (the Middle Urals) and Ni-enriched soil from an area of a natural geochemical anomaly (the Polar Urals). A. cepa roots were exposed to aqueous soil extracts for $30 \mathrm{~h}$ with recovery duration for 20 and $44 \mathrm{~h}$. Nuclear anomalies were observed in exposed root meristematic cells. High $\mathrm{Ni}$ concentrations decreased the roots growth along with $\mathrm{MN}$ inductions. The total number of anomalies and contribution of extrusions among them were higher for contaminated soils with prevalence of $\mathrm{Cu}$. Masood and Malik (2013) collected and extracted soil samples from agricultural fields in the vicinity of industrial area of Jajmau, Kanpur (India), using dichloromethane (DCM) and hexane solvents, and subjected to genotoxicity testing using $A$. cepa CA assay. All the tested concentrations of soil extracts (5-100\%) affected mitotic index in a dose-dependent manner and among CA's, Cmitosis, anaphase bridges, laggards, binucleated cells, stickiness, broken and unequal distributions of chromosomes at anaphase stage of cell division were observed. Based on results, it seems that large number of pollutants has been accumulated in soil and might have adverse impact on soil health. Oberholster et al. (2008) studied the Macroinvertebrate communities in Rietvlei nature reserve wetland area and their relationship with water quality. Samples were collected from sewage effluent, agricultural and informal residential runoff. A large increase in nutrient concentrations was observed downstream from discharged treated sewage with an associated decrease in species richness. A. cepa along with other bioassays (D. magna, $H$. attenuate, L. sativa, P. adspersus) showed toxicity and authors revealed the deterioration of the wetland possibly due to factors such as increasing urbanization, industrialization, agriculture runoff and rapid human settlement in the Hennops River catchment area and its principal tributaries. Christofoletti et al. (2013) assessed the toxic potential of biosolid, obtained from a sewage treatment plant, vinasse (a by-product of the sugar cane industry), and a combination of both residues using $A$. сера assay. $A$. сера test revealed genotoxic since frequesnt CA's were observed. Micronuclei and chromosome breaks on meristematic cells and $\mathrm{F}$ cells with micronuclei were examined to assess mutagenicity of samples. After 30 days, the genotoxic effects were significantly reduced in the soil + biosolid and soil + biosolid + vinasse groups as well as the mutagenic effects in the soil + biosolid + vinasse group. Based on results, authors suggested bioprocessing of residues by diplopods as feasible alternative prior to application to crops to improve degraded soils and/or city dumps.

While studying the presence of potential toxic chemicals in water sources of Nën-Shkodra lowland agricultural area after massive 2010-2011 flooding, Kopliku and Mesi (2012) employed A. cepa root tips assay considering root length, MI, phase index and CA endpoints. Roots were grown in four water samples, analysed and compared with controls. Inhibition of root growth, cell division and induction of mitotic and CA's were observed. The most polluted water sample caused inhibited root growth up to $36 \%$, MI decreased up to $38 \%$, and CA's up to 7.8\%. Among CA's, bridges and fragments, stickiness and Cmetaphase were observed. The results indicated a slight toxic tendency of analyzed natural waters and A. cepa served as an alert of chemical pollution after flooding.

\section{Sugar Industry}

The onion bulbs were treated with different concentrations of the distillery effluent $(25,50,75$ and 100\%) at room temperature (Hemanth Kumar et al., 2015) and resultantly, $\mathrm{MI}$ and relative division rate were decreased in concentration dependent manner. The chromosomal abnormalities (abnormalities include sticky metaphase, disturbed chromosome, sticky telophase, multipolar chromosome, laggards, fragmented metaphase, fragmented anaphase, scattered anaphase and chromosomal bridge) also increased as the concentration of the effluent increased. A. cepa test showed that the higher concentrations of distillery effluents inhibited cell division significantly. In another study, PedroEscher et al., studied vinasse (by-product of the biomass distillation, mainly for the production of ethanol, from different cultures such as sugarcane and used as fertilizer) for toxicity using A. cepa test (Pedro-Escher et al., 2016). using $A$. серa, bulbs were exposed to raw vinasse (RV) (from two different harvests (I and II)) and control soil + raw vinasse (SV); vinasse diluted in water at $50 \%+$ control soil (V $50 \%$ ); vinasse diluted in water at $25 \%+$ control soil (V 25\%); vinasse diluted in water at $12.5 \%+$ control soil (V $12.5 \%$ ). The chemical characterization of vinasse samples showed a low $\mathrm{pH}$ and high concentration of potassium. The results demonstrated that the two RV samples tested are toxic, since no germination was observed. The cytotoxic potential was observed in the sample II of SV and V (50\%). All groups evaluated in samples I and II, induced chromosomal alterations. SV (Sample I) and all groups evaluated in samples II induced MN. Based on toxicity results, author warned not to use vinasse as fertilizer since it can cause genetic damage. Similarly, the genotoxicity of four soil types collected from different regions of Amritsar, India employing chromosomal 
aberration assay in root tip cells of $A$. cepa has also been studied (2014) (Soodan et al., 2014). Roots exposed to different types of soil revealed the CA's like physiological aberrations (c-mitosis, delayed anaphases, stickiness, laggards, vagrants) and clastogenic aberrations (chromosomal breaks, chromatin bridge and ring chromosomes). These studies revaled that sugar industry waste is also potential source of toxicity and A. cepa test efficiently applied for toxicity monitoring.

\section{Disinfected water and mineral water/tap water}

Disinfection of drinking water is one of the most important steps in the potabilization process, since it ensures microbiological risk control associated with water consumption. Nevertheless, the use of disinfectants in this process can increase chemical reactions with substances naturally present in the water or with pollutants, generating disinfection by-products, for which the genotoxic and carcinogenic activities have been reported (Boorman, 1999; Feretti et al., 2008; Leme and Marin-Morales, 2009; Melnick et al., 1994). For example, super-chlorination the production of chloroform and trihalomethanes along with haloacetic acids and haloacetonitriles were identified (Moudgal et al., 2000) and the use of $\mathrm{ClO}_{2}$ in the potabilization process has increased now a days and by mixing with water is reduced to chlorite $\left(\mathrm{ClO}_{2}^{-}\right)$and to chlorate $\left(\mathrm{ClO}_{3}^{-}\right)$ions, for which the adverse effects to the exposed organisms are not totally understood (Leme and Marin-Morales, 2009). The wastewater disinfection helps prevent the spread of pathogens and chlorination is the most widely used method for the disinfection of wastewater, but it can cause the formation of mutagenic, carcinogenic and toxic by-products which are potentially harmful to human and aquatic organisms (Monarca et al., 2000). In this regard, the disinfectant agent alternative to chlorine, such as chlorine dioxide, ozone, peracetic acid and UV radiation, on the formation of mutagenic and toxic compounds in wastewater has been studied. Wastewater samples were collected before and after disinfection in summer and in winter to detect genotoxicity using $A$. серa test and found higher $\mathrm{MN}$ frequency in peracetic acid treated wastewater sampled in winter. The same author in subsequent year reported the genotoxicity of surface water treated with different disinfecting agents. The toxicity assays (MN and CA tests) were performed to detect the clastogenic and aneugenic effects. The study was carried out at a pilot plant using lake water after sedimentation and filtration. This water supplied four stainless steel basins: three basins were disinfected with sodium hypochlorite, chlorine dioxide, and peracetic acid and the fourth basin containing untreated lake water was used as a control. A. cepa was exposed in situ in the basins and resultantly, the $A$. cepa showed $\mathrm{MN}$ and CA in roots treated with disinfected water (Monarca et al., 2003).

The CA test was applied in order to evaluate the toxicity of the $\mathrm{ClO}_{2}-$ and $\mathrm{ClO}_{3}{ }^{-}$ions. The authors reported positive results for the $A$. cepa test, concerning a significant CA induction by $\mathrm{ClO}_{2}^{-}$and $\mathrm{ClO}_{3}-$ ions, even in concentrations below the limits established by the Italian normative law $(0.2$ $\mathrm{mg} / \mathrm{L}$ ) and by the World Health Organization (WHO) guidelines $(0.7 \mathrm{mg} / \mathrm{L})$ (Feretti et al., 2008). While studying the properties of mineral water packed in polyethylene terephthalate (PET) bottles, Evandri et al. (Evandri et al., 2000) revealed the toxicological effects of mineral water packaged on the basis of $A$. cepa test. Two commercial mineral water brands, bottled both in PET and glass, stored under different conditions (no direct light exposure, storage water $\left(40^{\circ} \mathrm{C}\right.$ for 10 days in the dark) and exposure to sunlight at varying temperatures. After assayed by $A$. серa, the root length and color were affected. The water samples bottled in PET induced cytogenetic aberrations and the signs of toxicity were evident even after 8 weeks of bottling, which is well within the recommended expiry date and suggested that storage conditions were very important, as that CA's were particularly apparent after exposure to direct sunlight. Crebelli et al. (2002) analyzed the genotoxic and ecotoxicologic effects of urban wastewater disinfected with sodium hypochlorite or peracetic acid. The mutagenic activity of disinfected effluents was similar to the corresponding untreated wastewater both sampled in four different periods. Therefore, the disinfection process did not seem to contribute to aquatic mutagenicity in the examined range of biocide concentration. Dissociation compounds (hydrogen peroxide and acetic acid) and possible byproducts of peracetic acid did not seem to contribute significantly to the toxicity of sewage treated with peracetic acid.

In contrary to above reports Crebelli et al. (Crebelli et al., 2005) investigated the influence of two widely used disinfectants, peracetic acid and sodium hypochlorite $(\mathrm{NaClO})$, on the formation of mutagenic by-products. After disinfection, the water samples were tested for toxicity $A$. cepa root (anaphase aberration) and no negative impact was observed in the $A$. cepa anaphase aberration test. These results indicate that, in the conditions applied, wastewater disinfection with PAA and $\mathrm{NaClO}$ does not lead to the formation of significant amounts of toxic by-products. Furthermore, Egito et al. (Egito et al., 2007) also reported non toxicity in chlorinated water used for human consumption.

Goncharuk et al. (2011) tested tap water treated in static conditions with gray and black silicon mineral using $A$. cepa test. Silicon-activated water stimulates metabolic processes during the germination and root growth was stimulated, which confirmed the sensitivity of $A$. серa for toxicity evaluation and Silicon-activated water was found to be safe resulting in the stimulation of the growth and development. In another study, Marrakesh's CMR wastewater, Draa Lasfar mine located about $12 \mathrm{~km}$ southWest of the city of Marrakech, Morocco for toxicity using $A$. cepa assay was screened (Chaik et al., 2011) and results revealed that wastewater at $100 \%$ concentration inhibited root growth and $\mathrm{MI}$; induced binucleated cells, but was nontoxic at very low concentrations. Un-controlled cell division led to polyploidy. The wastewater showed the 
presence of $\mathrm{Zn}, \mathrm{Pb}, \mathrm{Cu}$ and $\mathrm{Cd}$, whose presence could partly be responsible for the toxicity of wastewater. Author's concluded that water should be used at very low concentrations in order to protect the ecosystem from any potential adverse effects. Olorunfemi (2013) investigated the cytotoxic and genotoxic potentials of borehole water supply in six halls of residence of the University of Benin main campus using $A$. cepa test. The tested samples inhibited the root growth and induction CA's, which were correlated with water physico-chemical nature and heavy metals present in high concentrations in the borehole water samples than tap water. Findings suggested that the investigated water samples may contaminate by wastewater from sewage and solid waste leachates and may cause health risk in case of exposure during consumption. Bohórquez-Echeverry et al. (2012) compared various bioassays (five acute toxicity bioassays) to monitor the quality/toxicity of the affluent and effluent of three drinking-water treatment plants. Results revealed that all organisms were potentially useful in the assessment of water quality. Olorunfemi et al. (2014) monitored the rural settlements area (Obazuwa community in Ovia North East Local Government Area of Edo State, Nigeria) water source (lake water during the dry season, lake water during the wet season and borehole water) for physico-chemical and cyto-genotoxic properties. The physico-chemical analysis revealed the $\mathrm{pH}$, chromium, copper, chlorides, nickel, iron, zinc, cadmium, lead and manganese in lake water in both seasons higher than WHO permissible limits. The MI values in exposed roots decreased along with CA's (bridges, fragments, sticky chromosomes, disoriented chromosomes) and MN inductions.

The cytotoxicity and genotoxicity in selected borehole water using $A$. cepa assay was also studied. Roots were exposed to five different water samples collected from boreholes Ring road, Sakponba, Ekosodin, Science and Eddy Grace in Benin City, Edo State, Nigeria for 96-h and results clearly shown that water and environmental pollutants in Benin metropolis is reached to high level (Adelanwa et al., 2011). Bandyopadhyay (Bandyopadhyay, 2015) evaluated arsenic contaminated groundwater from Eastern region of Burdwan district, West Bengal. Treatment for 4 days of newly developed roots of $A$. сера with water samples with arsenic content $50 \mu \mathrm{g} / \mathrm{L}$ exhibited stimulation in mitotic activity whereas samples with arsenic $1000 \mu \mathrm{g} / \mathrm{L}$ showed inhibition of mitotic activity apparently indicating hormesis. Results indicated that contaminated groundwater depending on the magnitude of the arsenic concentration, might either be mitogenic or mitostatic/toxic, which in turn has obvious implications in agriculture and human health.

\section{Radioactive wastes contaminated soil and water bodies}

The soil and water bodies contaminated with radioactive material also revealed the toxic effect (Kovalchuk et al., 1998). There are few studies in the literature on the evaluation of the hazards of radioactive soil and watershed contamination. However, available studies report the sensitivity of the A. cepa to assess this pollutant class. The CA test was applied to evaluate the genotoxic potential of watershed near radium production industry storage cell. The concentration of radioactive material $\left({ }^{262} \mathrm{Ra},{ }^{228} \mathrm{U},{ }^{232} \mathrm{Th},{ }^{210} \mathrm{~Pb}\right.$ and ${ }^{210} \mathrm{Po}$ ) in all samples were found below the permissible limit. However, the heavy metal ions ( $\mathrm{Zn}$ and $\mathrm{Mn}$ ) concentration exceeded the maximum permissible concentration for the natural reservoirs. All water samples showed a significant increase in CA's frequency as compared to control. The regression analysis revealed an increase in MI parallel to $\mathrm{Zn}$ ion levels, while MI decrease was correlated with ${ }^{238} \mathrm{U}$ higher concentrations. Authors suggested the combination of two methods, toxicity evaluation and estimation of chemical composition of the system under test, which allows the identification of the elements that require constant biological monitoring such as $\mathrm{Zn}$ and ${ }^{238} \mathrm{U}$ which were found most effective regarding genotoxicity in $A$. серa root (Evseeva et al., 2003). Later, Evseeva et al. (2005) also studied the mutagenic and cytotoxic effects of concentrations of cadmium, potassium and thallium-232 in A. cepa root meristem. The combined action of ${ }^{232} \mathrm{Th}(0.8 \mathrm{mM})$ with cadmium in non-toxic $(0.009 \mathrm{mM})$ and toxic $(5 \mathrm{mM})$ concentrations resulted in sinergetic increase of the frequency of aberrant cells in $A$. серa root meristem. Decrease of the mutagenic effect to the additive level and antagonism with respect to the cytotoxic one was observed only at the certain concentrations of ${ }^{232} \mathrm{Th}(0.8 \mathrm{mM})$ and $\mathrm{Cd}$ $(0.09 \mathrm{mM})$ and the time of impact $30 \mathrm{~h}$. In contrast to the heavy metal cadmium the essential for plants potassium at all studied concentrations $(0.008,6,13 \mathrm{mM})$ decreased the number of cytogenetic aberrations in control experiments and under the effect of ${ }^{232} \mathrm{Th}$. The maximum protective effect of potassium was detected at the concentration $13 \mathrm{mM}$. In another study, while studying the genotoxic effect of radioactive explosion, Ukraine (Chernobyl accident), applied the CA test in A. cepa root meristematic cells to estimate the soil contamination, contaminated by cesium $\left({ }^{137} \mathrm{Cs}\right)$ and strontium $\left({ }^{90} \mathrm{Sr}\right.$ ) (Kovalchuk et al., 1998). Authors showed a dose-dependent relationship between the increase in the percentage of aberrant mitosis and the increase in the radioactive contamination in the soil samples and the same relationship was verified for MI inhibition. The authors also depicted a strong correlation between the ${ }^{137} \mathrm{Cs}$ activity and the cytotoxic and genotoxic action observed. Finally, they concluded that the A. cepa test is efficient and sensitive to evaluate the genotoxicity of soils impacted by radioactive pollutants. Udalova et al. (2014) evaluated the cytotoxicity and genotoxicity of natural waters contaminated with ${ }^{90} \mathrm{Sr}$ and heavy metals in the vicinity of the radioactive waste storage, Obninsk, Kaluga region. Exposed root analysis showed mutagenic potential of waters collected in the vicinity of the radioactive waste storage. Saghirzadeh et al. (2008) estimated the impact of high level of natural radiation in the soils of inhabited zones of Ramsar, Iran using DNA damage in $A$. cepa. The average specific activity of natural radionuclides measured in the soil samples for ${ }^{226} \mathrm{Ra}$ was $12,766 \mathrm{~Bq} \mathrm{~kg}^{-1}$, whereas in the control soils it was in the range of $34-60 \mathrm{~Bq} \mathrm{~kg}^{-1}$. A positive strong significant 
correlation of the DNA damage in nuclei of the root cells of $A$. cepa seeds germinated in the soil of high background radiation areas with ${ }^{226} \mathrm{Ra}$ activity of the soil samples was observed. The results showed high genotoxicity of radioactively contaminated soils. While assessing the cytogentic activity of water from a regional radioactive waste repository (Pyatkova et al., 2009), A. cepa test was applied. Biotesting of natural waters from the site has demonstrated that negative biological effects were generated under the influence of water from well, located near the source of contamination. The percentage of aberrant cells in water samples from well 4 was 2 times higher than in the control or other sources. Effects of water composition at the genome level were found season independent. The Allium-test was shown to be highly sensitive and effective in testing a combined effect from radiation and chemical factors in field conditions.

\section{Complex mixtures}

Complex mixtures characterized most of the environmental samples, since they can suffer the influence of several contamination sources. Thus, several studies have been conducted by the $A$. cepa test to evaluate toxicity. In order to evaluate the potential mutagenic effect of raw and simulated leachate from a rural refuse dump site at Odo Oba, SouthWest Nigeria, Bakare (2001) applied A. cepa MI and CA end points. Roots of about $2-3 \mathrm{~cm}$ long were treated with $1 \%$, $2.5 \%, 5 \%, 10 \%$ and $25 \%$ concentrations of the leachate for 24 h. analysis showed different types of CA's at all doses tested except at $1 \%$ concentration. The number of dividing cells also reduced at the tested concentrations. Results revealed the genotoxic chemicals found in the leachate samples and findings were foundvery useful in the practical aspects of waste management and for the assessment of the hazardous effects of the chemicals in the leachate from solid waste dumpsites. Similarly, metalworking fluids (MWFs) EC50 values using the $A$. cepa test at $1 / 250,1 / 500$ and $1 / 1000$ concentrations were also studied using $A$. cepa assay (Pekol, 2014). Decrease in MI as well as CA's (stickiness were observed to occur; whereas abnormalities such as c-mitosis, fragments, bridges, vagrants) alongwith MN inductions were observed in concentration dependent manner. Feretti et al. (2008) evaluated the clastogenic effects of gaseous emissions (biogas) from three municipal landfills using in situ $A$. cepa test. Leachate samples inhibited root growth and tip development in A. cepa. Genotoxicity of the leachates was evaluated in diluted samples and a significant increase in chromosomal metaphase aberrations in one of the samples was observed. Biogas was nontoxic, whereas leachates were found to display elevated toxicity and author suggested to treat leachates before releasing into the environment to avoid ecological damages. Pelegrini et al. (2007) evaluated toxicity in natural leachate samples collected from Limeira Sanitary Landfill, Limeira, Brazilian state of São Paulo using A. cepa along with other test organisms. A. cepa (onion) indicated that the diluted leachate up to $50 \%$ showed similar toxicity to the phenol solution $2000 \mathrm{mg} / \mathrm{L}$ and $\operatorname{Cr}(\mathrm{VI})$ concentration of $2000 \mathrm{mg} / \mathrm{L}$. Obidoska and Jasińska (2008) monitored the toxic and genotoxic potential of municipal landfill leachates from Radiowo municipal, Poland and evaluated the efficiency of zeolite as a leachate purifier using A. cepa assay. A significant phytotoxicity and genotoxicity of Radiowo landfill leachate was observed and zeolite filtration did not reduce genotoxicity, however, phytotoxicity was decreased. Oberholster et al. (2008) studied macroinvertebrate communities in Rietvlei nature reserve wetland area and their relationship with the aim to evaluate their use as potential indicators of pollution. Samples were collected from outlets of sewage effluent, agricultural and informal residential runoff. A. cepa bioassay was performed for toxicity evaluation along with other assays (D. magna, $H$. attenuate, $L$. sativa and $P$. adspersus). Multitrophic level bioassays revealed the deterioration of the wetland possibly due to factors such as increasing urbanization, industrialization, agriculture runoff and rapid human settlement in the Hennops River catchment area and its principal tributaries.

While evaluating the efficiency of biological and chemical wastewater treatment plant Sik et al., showed that after wastewater treatment a significant reduction in MI and CA was observed (Sik et al., 2009). For comparison, the $A$. cepa were kept in 100\% concentrations of the treated water and $10 \%, 25 \%, 50 \%$ and $100 \%$ concentrations of untreated water and a significant difference was noted. Wastewater reduced the rate of the mitotic division at different concentrations and increased the mitotic anomalies. The MI was found to be $33.8 \%, 31.2 \%, 23.6 \%$ and $16.7 \%$ in the control, treated water, $10 \%$ and $25 \%$ concentration of the untreated water, respectively and similar rates in mitosis for four groups of plants were observed. The CA's as high frequency of lagging chromosome, irregular distribution, polar slips, horizontal division and sticky chromosome were also observed in group of plant exposed to untreated wastewater and authors suggested the use of biological and chemical wastewater treatment before being discharged into watershed. El-Shahaby et al. (El-Shahaby et al., 2003) applied the CA and MN test in A. cepa to evaluate the genotoxicity of industrial effluents collected from Sandub area, Dakahlia, Egypt. High genotoxicity was reported for all the water samples and correlated with the presence of some heavy metals ( $\mathrm{Pb}, \mathrm{Zn}, \mathrm{Co}, \mathrm{Cd}$ and $\mathrm{Cu}$ ). In this study, the authors concluded that the $\mathrm{CA}$ and $\mathrm{MN}$ tests in $A$. cepa are recommended for aquatic pollution biomonitoring due to its sensitivity to detect mutagens in wastewater. While studying the toxicity of industrial effluents of two cities in India contaminated mainly by heavy metals and pesticides, Fatima and Ahmad (2005) showed a significant increase in the CA frequency in $A$. cepa meristematic cells exposed to these samples. Waste sludge was analysed using $A$. серa genotoxicity test. Samples collected during three winter periods from three Danish municipal wastewater treatment plants differing in size and industrial load were tested. The toxicity of the sludge was tested using A. cepa root inhibition assay, and the results were expressed as EC30 and EC50. 
Results showed that the toxicity correlated positively with industrial load. However, when genotoxicity was tested at concentrations corresponding to the EC30 and EC50 values in the $A$. сера anaphase-telophase assay, only two sludge samples from the smallest plant with the lowest industrial load induced significant chromosome aberrations. Concentrations of the heavy metals $\mathrm{Pb}, \mathrm{Ni}, \mathrm{Cr}, \mathrm{Zn}, \mathrm{Cu}$, and $\mathrm{Cd}$ were also determined and could partly be correlated with the toxicity of the sludge and the industrial load of the treatment plants (Rank and Nielsen, 1998).In another study Carita and Marin-Morales studied (Carita and Marin-Morales, 2008) sludge from different urban sewage treatment stations in southeastern Brazil, and reported sensitivity of the A. cepa test to assess the genotoxic and mutagenic potentials of the sludge. The different responses may be related to the preparation of the test samples, e.g. solubilization procedures to obtain aqueous extracts, during which some chemicals may be lost or even retained in the raw material, depending on the procedure used. Furthermore, the difference in observations may also be related to the type of sewage in each studied region.

An assessment of the vermicompost efficiency, a bioremediation process, used to reduce toxic potential of a municipal sludge sample, in which heavy metals were detected, was made by some authors (Srivastava et al., 2005). MI inhibition for raw sludge and an increase of this parameter in the bioremediated sludge were reported by the $A$. сера test system. The exposition of $A$. cepa roots to raw sludge also induced significant CA frequencies, which was not observed for bioremediated sludge. In this way, the authors showed that vermitechnology can be a good technique to remediate municipal sludge. Pseudomonas fluorescens strain isolated from the soil of industrial estate of Aligarh, India, which was resistant to $\mathrm{Cd}^{2+}, \mathrm{Cr}^{6+}, \mathrm{Cu}^{2+}, \mathrm{Ni}^{2+}, \mathrm{Pb}^{2+}, \mathrm{BHC}, 2,4-\mathrm{D}$, mancozeb and phenols up to high level and for the detoxification pollutants. The decrease in toxicity determined by the $A$. cepa test was $62.5 \%$ for the model water containing the mixture of test heavy metals, $71.9 \%$ for the pesticides, $73.2 \%$ for phenols, and $58.5 \%$ for combination of all these pollutants. The efficiency of bioremediation for certain heavy metals at the same concentrations by means of immobilized cells of the test Pseudomonas fluorescens isolate was estimated to be $75.9 \%$ for cadmium, $74.2 \%$ for hexavalent chromium and $61.0 \%$ for lead during the 24 hours' treatment. Authors concluded that isolated strain was highly effective to degrade the pollutant and A. cepa was efficient to estimate toxicity before and after treatment (Khan and Ahmad, 2006). The municipal wastewater is composed of highly varied substances with considerable contents of toxic agents (White and Rasmussen, 1998) and several authors reported the cytotoxicity, genotoxicity and mutagenicity by $A$. cepa test system. In order to assess the toxicity of domestic wastewater Bianchi et al., reported a significant induction of CA's, MN, cell death and inhibition of the M I (Bianchi et al., 2011). The chemical analysis revealed the presence of $\mathrm{Pb}$, which may be responsible for the effects observed in A. cepa cells. In a study Kwasniewska et al., assessed (Kwasniewska et al., 2012), the genotoxicity of aqueous leachates from municipal solid waste landfill sites in Southern Poland. Six leachate samples out of 22 showed cell divisions abnormalities and the authors revealed more sensitivity of $A$. cepa as compared to Ames test for leachates genotoxicity evaluation. In another study, the municipal wastewater treatment plants sludge was found to be genotoxic to A. cepa (CA's) and chemical analysis revealed the presence of heavy metals ( $\mathrm{Pb}, \mathrm{Ni}, \mathrm{Cr}, \mathrm{Zn}, \mathrm{Cu}$ and $\mathrm{Cd}$ ), which may be responsible for CA's in A. cepa (Rank and Nielsen, 1998), whereas SmakaKincl et al., reported (Smaka-Kincl et al., 1996), that the municipal waste water effects on root growth, MI and MN up to $74 \%, 66 \%$ and $3 \%$ and aberrant cells were also observed in $A$. cepa root cells and Nielsen and Rank (Nielsen and Rank, 1994) observed the CA's in A. cepa root cells exposed to treated municipal wastewater for five days. Mesi and Kopliku (2011) monitored the toxicity of some Malësia e Madhe aqueous sources by root length, form, turgescence, color and MI end points. Roots were exposed to water from Dobër wells, Vraka runnel, and Shegan waterside. Along with change in root macroscopic properties, the MI values decreased in following order, distilled water, CuSO4 solution, acetone solution, Shegan, Vraka, Dobër.

While studying the possible toxicity of sludge in view of its use in agriculture, Christofoletti et al. (2012) studied biosolid sample toxicity under laboratory conditions up to 30 days using diplopods Rhinocricus padbergi and plants Allium cepa (onion) as test organisms. The data obtained demonstrated that the biosolid raw sample had genotoxic potential. A. cepa analysis showed genotoxicity, but this effect was reduced after 30 days of bioprocessing by diplopods. Study highlighted the importance of $A$. сера as well as waste management for application to agriculture soil to avoid toxic effects on soil as well as plants. In order to asses the genotoxicity of sewage effluent, Ukaegbu and Odeigah (Ukaegbu and Odeigah, 2009) applied the morphological and CA assay. There was a significant decrease in root length of the experiment. Also the MI decreased as concentration increased and the total aberrations were also increased parallel to MI at higher concentration. The results demonstrated that the $A$. cepa test is a useful screening test for the evaluation of toxicity in sewage effluent. A study to evaluate the genotoxic effects of municipal wastewater by $A$. cepa test showed significant CA values and MI inhibition for all the test samples. In the $A$. cepa test, samples from four stages (crude sewage, primary, secondary and tertiary effluent) of the wastewater treatment plant was analyzed. The numbers of aberrant cells found in the $A$. cepa roots did not differ among the four stages tested. At all stages, the most concentrated sample was more toxic than the respective diluted samples, as demonstrated by the decreased MI (Grisolia et al., 2005). While studying the toxicity of leachates from municipal solid waste landfill sites in Southern Poland through various biological assays Kwasniewska et al., (Kwasniewska et al., 2012) revealed that aqueous leachates from municipal solid waste landfill affected $A$. cepa normal growth. Among the bioassays used in the study, the A. cepa 
test proved to be more sensitive than bacterial tests for the investigation of leachate toxicity. The results suggested that the cytogenetic bioassay was efficient and simple for genotoxicity studies of leachates and biological effects were correlated with chemical parameters that have a genotoxic potential against $A$. cepa. A study to evaluate the toxic effects of hospital incinerator bottom ash leachate by $A$. cepa root cells (Akinbola et al., 2011), applied the CA test at concentrations ranging from $1 \%$ to $50 \%$ of the leachates and results showed a significant inhibition in root growth and induction of CA's and effects were correlated with contents of heavy metals.

The mutagens in complex environmental mixtures i.e., surface water or industrial wastewater was detected near the Sava River, Croatia using A. cepa root assay (Radić et al., 2010). The morphology of the A. cepa roots, inhibition of root growth, cell division and induction of mitotic and CA end points were observed. The most highly polluted water samples (industrial effluents) caused an inhibition of root growth up to $50 \%$ and MI was decreased up to $40 \%$ along with considerable CA's as compared to control. In view of positive results, authors suggested the application of mutagenicity/genotoxicity assays along with conventional chemical analysis for quality monitoring to quantify the mutagens in surface and wastewaters. Also, the toxic impact of raw waste leachates from Shkodra city wastewater was investigated using A.сера test. Leachates were collected from Rusi vegetable market, University campus, centre city domestic and the main collector runoff to the lake open dump sites. The onion bulbs were grown in 1\%, 2.5\%, 5\%, 20\% concentrations and root length, EC50, MI and CA end points were studied. Growth was restricted in all leachate in increasing concentrations manner. The EC50 values were recorded as $2.9 \%, 3.5 \%, 5.5 \%$ and $6.2 \%$ for Rusi vegetable market, University campus, a centre city domestic and the main collector runoff to the lake open dump sites leachate samples. The genotoxicity and root growth inhibition showed linear relationship. Among CA aberrations, stickiness, chromosome bridges and fragments, laggard and vagrant chromosomes were observed (Mesi and Kopliku, 2012). Bakare et al. (2012) investigated the toxicity using raw and simulated e-waste leachates by $A$. cepa. Roots were exposed to $1,5,10,25$ and $50 \%(\mathrm{v} / \mathrm{v}$; leachate/tap water) concentration of each of the leachate sample. Cytogenetic and root length inhibition analyses were evaluated at 48 and 72 $\mathrm{h}$, respectively. The root growth and cell proliferation inhibited dose-dependently. The leachates also induced morphological modifications in the roots and CA's (anaphase bridge, sticky chromosomes and binucleate cells) and toxicity was correlated with $\mathrm{Fe}, \mathrm{Cd}, \mathrm{Mn}, \mathrm{Cu}, \mathrm{Ni}, \mathrm{Cr} \mathrm{As}, \mathrm{Zn}$ and $\mathrm{Pb}$ metals. These observations indicate that e-waste leachate contained cytotoxicity and mutagenic agents, which might be the environmental and public health issue in view of e-waste exposure. Shashank and Suresh (2013) collected sewage effluent form Mandsaur (Madhya Pradesh), characterized (physico-chemical) and subjected to toxicity test. A. cepa roots were treated with different concentrations for 10 days and the roots length was measured every day, decreased when the effluent concentration increased. A. cepa showed better growth at $20 \%$ dilution of water and authors suggested the use of diluted water for irrigation since toxicity was observed at concentrated wastewater. do Canto et al (2013) evaluated the toxicity of landfill leachate in the city of Araranguá (Santa Catarina state, Brazil) using A. cepa bioassay. Roots were exposed to leachate for 7 days and the phytotoxic effect was evaluated by determining the root growth and mass of the roots, and the mass gain of the bulbs. The results indicated that the leachate significantly affected the root growth and mass of the roots and the mass gain of the bulbs. Leachate has toxic potentials to A. cepa that were associated with the presence of contaminants in solution. Klauck et al. (2013) assessed the toxic and genotoxic effects of an untreated leachate sample. The leachate was collected in a landfill located in the region of Vale do Rio dos Sinos, southern Brazil. The bulbs of $A$. cepa were exposed to concentrations of $5 \%, 10 \%, 25 \%, 50 \%$ and $100 \%$ for $48 \mathrm{~h}$. Results showed high toxicity, with significant root growth retardation as well as reduction in MI in bulbs exposed to $100 \%$ concentration of the leachate. The CA like abnormalities in anaphase-telophase was observed in accordance with increase in the concentration of leachate (5\%, 10\%, 25\% and 50\%). Andrade-Vieira et al. (2012) evaluated the cytogenetics effect of Spent Pot Liner (SPL) (a complex solid waste from the aluminum industry, composed of organics, fluoride salts, inorganic cyanides, metals, and sodium) using A. cepa bioassay. Roots were exposed to 0,10 , and $25 \%$ concentrations of leachates from SPL-soil mixtures for $4,8,12,24$, and $36 \mathrm{~h}$. The results showed an overall mitodepressive effect accompanied by an increased percentage of condensed nuclei and genomic instability as evidenced by cellular and CA inductions.

Garaj-Vrhovac et al. (2013) investigated landfill leachate from the Piškornica (Croatia) sanitary landfill treated by different methods. Chemical treatment procedure combined with chemical precipitation with $\mathrm{CaO}$ followed by coagulation with ferric chloride and final adsorption by clinoptilolite. Electrochemical treatment approach included pretreatment with ozone followed by electrooxidation/electrocoagulation and final polishing by microwave irradiation. Cytotoxic effect of the original leachate was obtained for both exposure periods ( 4 and $24 \mathrm{~h}$ ) while treated samples showed no cytotoxic effect even after prolonged exposure time. Both methods were found suitable for the treatment of complex waste effluent due to high removal efficiency of all measured parameters for toxicological safety of the treated effluent. Grisales Penagos et al. (2012) evaluated the removal of the organic matter present in hospital effluent by ozonation and in response to $70 \%$ biodegradability increment, the acute toxicity decreased by $62 \%$, which revealed sensitivity of A. cepa and efficiency of ozonation for hospital wastewater treatment.

Treated hospital effluent applying ozone at different $\mathrm{pH}$ conditions $(3,0,6,7,10)$, and other parameters such as UV254, biodegradability ratio (COD/BOD) and color (VIS436) 
were measured along with toxicity monitoring (Dayana et al., 2012). In response to biodegradability of $70 \%$ the toxicity decreased by $62 \%$. The ozone application seems to be a viable alternative to treat hospital effluents as a pretreatment of a biological process and $A$. cepa was to be sensitive to evauate biodegradability. Similarly (Kern et al., 2013), treated hospital laundry wastewaters by advanced oxidative processes (photocatalytic ozonation $\mathrm{O}_{3}, \mathrm{UV}, \mathrm{UV} / \mathrm{O}_{3}$, $\mathrm{UV} / \mathrm{O}_{3} / \mathrm{Fe}^{2+} 50 \mathrm{mg} / \mathrm{L}$ and $\left.150 \mathrm{mg} / \mathrm{L}\right)$. The analysis of the investigated wastewater revealed high chemical oxygen demand (COD-3343.8 mg/L, biochemical oxygen demand (BOD $5-1906.4 \mathrm{mg} / \mathrm{L}$, total Kjeldahl nitrogen (TKN - 79.8 $\mathrm{mg} / \mathrm{L}$. The treated and un-treated wastewater was tested for genotoxic using $A$. cepa. After treatment COD (59.1\%), BOD 5 $(50.3 \%)$ and TKN $(86.8 \%)$ were reduced. Normalization of the $\mathrm{MI}$ and reduction of micronucleated cells were observed in A. cepa after the treatments. Results demonstrated that these methods were efficient in the degradation of hospital laundry wastewaters, representing a thriving alternative for the removal of pollutants that caused toxicity and genotoxicity and A. cepa was found an efficient test to detect the toxicity load before and after treatment. Roig et al. (2012) studied the chemical and ecotoxicological properties of 28 sewage sludge samples from Spanish wastewater treatment plants in order to assess their suitability for agricultural purposes. Sludge samples were classified into five categories according to specific treatment processes in terms of digestion (aerobic/anaerobic) and drying (mechanical/thermal). Composted samples, as indicative of the most refined process, were also considered. The concentrations of seven metals ( $\mathrm{Cd}, \mathrm{Cr}, \mathrm{Cu}, \mathrm{Pb}, \mathrm{Zn}, \mathrm{Ni}, \mathrm{Hg}$ ) and organic substances (phenolic compounds, polycyclic aromatic hydrocarbons, polychlorinated biphenyls, polychlorinated naphthalenes, polybrominated diphenyl ethers, and perfluorinated compounds) were present in samples. Root elongation test with A. cepa along with other test organism was conducted. Significant differences were found in the following parameters: dry matter, electrical conductivity, nitrogen, organic matter and its stability, phytotoxicity and ecotoxicity, depending on the sludge treatment. In turn, no significant differences were found between categories in the concentrations of most metals and organic pollutants, with the exception of free phenolic compounds. Furthermore, no correlation between total heavy metal burden and ecotoxicity was observed. However, a good correlation was found between phenolic compounds and most ecotoxicological tests. These results suggested that sludge stability (conditioned by sludge treatment) might have a greater influence on sludge ecotoxicity than the pollutant load. Composting was identified as the treatment resulting in the lowest toxicity. Magdaleno et al. (2008) tested Matanza-Riachuelo river sediment composition and genotoxicity in order to develop a cost-effective, environmentally sound option for disposal and management of contaminated dredged materials. Sampling was performed in a rural area, in a solid waste dumpsite and an urban and industrial area. The concentrations of total heavy metals increased from the upper basin to the lower basin. The concentrations of copper, lead, and chromium in the leachates exceeded the permissible levels for the protection of aquatic life. CA's were observed in $A$. cepa roots exposed to sediment samples and authors classified sediments as genotoxic hazardous waste.

The genotoxicity of industrial wastewater samples from Aligarh and Ghaziabad cities using A. cepa test has also been studied. Samples from both sites induced anaphase aberrations. Fragmentation of the chromosome was the predominant effect of the Aligarh water sample, wherease sample from Ghaziabad induced chromosome stickiness and correlated the toxicity with heavy metals and pesticides in water samples (Fatima and Ahmad, 2006a). Fatima and Ahmad (2005) also investigated heavy metals (Cd, $\mathrm{Cr}, \mathrm{Cu}, \mathrm{Hg}$, $\mathrm{Pb}$ and $\mathrm{Zn}$ ) pollution in water using $A$. cepa test system and positive results were obtained in parallel to other biassays used. Geras'kin et al. (2011) evaluated genotoxic potential of two environmental compartments (water and sediment) from the Upper Silesia Coal Basin (USCB), Poland by employing root meristem cells of $A$. серa. The clear genotoxic effect of water and sediment sampled was shown, with an important contribution of severe types of cytogenetic abnormalities. Results of simultaneous use of conventional monitoring methods and biological tests suggested that contemporary levels of persistent pollutants in post-mining areas of the USCB may enhance the risk both for human health and biological components of natural ecosystems.

Bio-remediation of pollutant have been found to be efficienct (Bilal et al., 2016a; Bilal et al., 2016e; Obek and Sasmaz, 2011; Sasmaz, 2008, 2009; Sasmaz et al., 2016a; Sasmaz and Obek, 2009, 2012; Sasmaz et al., 2008; Sasmaz and Sasmaz, 2009) and the effect on bio-treatment on toxicity have also been studied i.e., the efficiency of the aquatic macrophytes Salvinia auriculata Aublet in purification of polluted effluents, and toxicity was monitored by $A$. cepa test (2015) (Gonçalves et al., 2015). Three collections were performed in September 2011, the first analysis was performed with water collected directly from River Santa Catarina, Mexico and sampling was performed seven days after the contact of the effluent with the macrophytes, and then after fifteen days. According to the results of the A. test, the aquatic macrophyte $S$. auriculata was efficient in the removing of pollutants after fifteen days in contact with the effluent. While evaluating the performance of an Upflow Immobilized Biomass Anaerobic Reactor (UIBAR) for treating hospital wastewater, Porras Torres et al. (2013) applied $A$. cepa root growth inhibition test and toxicity was reduced up to $50 \%$ after treatment. The capacity of the anaerobic immobilized process to remove the compounds that produce toxicity was verified by $A$. cepa test.

Brkanac et al. (2014) assessed the toxic and genotoxic potential of leachate from an old sanitary landfill prior to and following chemical and electrochemical treatments by $A$. серa test. Untreated leachate inhibited $A$. cepa cell division and induction of mitotic and CA's before treatment and after treatment leachate did not show 
genotoxicity and electrochemical method was found to be more efficient in removing toxic substances present in landfill leachate and thus more suitable for treating such leachates prior to their discharge into the environment. A. cepa resonse of treated and un-treated leachate demonstrated that the ecotoxicity/genotoxicity assays should be used in leachate risk assessment together with physicochemical analysis. Pathiratne et al. (2015) assessed the efficacy of A. cepa test for screening cytotoxicity and genotoxicity of treated effluents originated from textile industries, rubber industry, and treatment plants of industrial zones. The heavy metal/metalloid levels of the effluents varied depending on the industry profile. In the A. cepa test system, the undiluted effluents induced significant root growth retardation, mitosis depression, and CA's. The results support the use of a practically feasible $A$. cepa test system for rapid screening of cytotoxicity and genotoxicity of diverse industrial effluents discharging into inland surface waters.

Klauck et al. (2015) evaluated leachate toxicity of a municipal solid waste landfill located in the Sinos River Valley region (southern Brazil). The roots of A. cepa were exposed to raw leachate and treated leachate (biological treatment). The levels of metals detected in both samples of leachate were low, and raw leachate showed high values for ammoniacal nitrogen and total Kjeldahl nitrogen. A. cepa showed a phytotoxic response to landfill leachate, showing reduced root elongation. The results indicated phytotoxicity in municipal solid waste landfill leachate, even after biological treatment. The cytotoxicity insecticides (Endosri \& Nuvan) and fungicide (Kvistin) using A. cepa root inhibition test at concentrations; Endosri $(2000,1000,500$, $250,125,60,30, \& 15 \mathrm{ppm})$, Nuvan $(400,350,300,250,200$, $150, \& 100 \mathrm{ppm})$ and Kvistin $(3000,2000,1000,750,500$, $250, \& 125 \mathrm{ppm}$ ) for 5 days was studied amd $\mathrm{EC}_{50}$ values obtained for Endosri, Nuvan and Kvistin were 60, 200 and 500 ppm, respectively. Both Endosri and Nuvan showed highly toxic effects and decreased in both root length and root count. Kvistin was less toxic and results indicated that toxicity could also contribute to several afflictions in other organisms after exposure to higher concentrations and a negative impact on the crop as well (Kuchy et al., 2015). While studying the nature of sewage sludge generated by wastewater treatment plants (WWTP), Mazzeo et al. (2015) applied A. cepa to evaluate the detoxification efficiency of treatment. Sewage studge/soil with 10,25 and 50\% proportions was buried for $0,2,6$ and 12 months in holes. Chemical analysis revealed the presence of high concentration of $m$ - and $p$-cresol, especially for samples analyzed after 0 or 2 months. Both, raw sewage studge and aqueous sewage studge extracts induced DNA damage in $A$. cepa, even when associated with soil. However, this effect was observed to decline during the attenuation period, although significant effects were detected for the highest tested concentration $(100 \%)$ even at the end of attenuation period. Based on A. cepa results, authors suggested the necessity of stabilization of sewage sludge and soil for a period of at least 12 months since $A$. cepa test proved to be sensitive tool to assess the efficiency of sewage sludge detoxification process. The physico-chemical characteristics of water from Dandugan Oya, a water canal located in the Western Province of Sri Lanka receives industrial waste from multiple sources along with toxicity using A. серa was assessed (Kannangara and Pathiratne, 2015a). Root exposed to wastewater and downstream water resulted in the reduction of root growth (24-62\%) and mitosis (31-55\%), MN (0.6\%), NA (3-14 folds) and CA's (3 - 21 folds) in downstream water. No significant difference between the control and the upstream water was found in relation to the measured biological effects. Results revealed that the tested wastewaters contained cyto-genotoxic agents and authors suggested using bioassays for water quality evaluation along physico-chemical analyses.

More recently, Papa et al. (2016) evaluated the performance of Wastewater Treatment Plants (WWTPs) using various toxicity tests i.e., algal growth inhibition, bioluminescence inhibition and acute toxicity test (baseline toxicity); an E-Screen-like assay (estrogenic activity); Ames, Allium cepa and Comet tests (mutagenic/genotoxic activity). All tests revealed good performance of WWTP which displayed the ability of WWTP to enhance effluent quality and efficiency of $A$. серa to detect the toxicity. Corrêa Martins et al. (2016) also reported the cytotoxic, genotoxic and mutagenic potential of sewage sludge using A. cepa bioassay. Solubilized and crude sludge from two sewage treatment stations were tested. Treated wastes showed less phytotoxicity, cytotoxicity, genotoxicity and/or mutagenicity. Despite negative results for MN F1 (micronuclei counted in F1 root cells, derived from meristematic cells) the authors recommended the use of genotoxic and mutagenic activities of sewage sludge to avoid negative effect on soil in view of their application to the agricultural soils. Recently, (Teena et al., 2016) also screened mutagenicity of complex environmental mixtures (surface water or industrial wastewater). Sewage effluents were collected from Kunnamkulam market, India and roots were exposed for 3, 6, 12, 24, and $48 \mathrm{~h}$. A significant CA (bridges, fragments, vagrant chromosomes, sticky chromosomes, Cmitosis, spindle abnormalities and disoriented chromosomes) along with decreased MI values were observed in exposure dependent manner. Authors suggested the treatment of sewage wastewater before use and toxicity evaluation using A. cepa test.

\section{Treated effluents/toxic compounds}

Industrial effluent contains toxic pollutants with elevated values of water quality parameters i.e., COD, color and heavy metals have been successfully treated using different methods. However, their might be generation of toxic byproduct, which needs monitoring using reliable assay and various researcher applied the $A$. сеpa test to monitor the efficiency of treatment methods (Ashar et al., 2016; Babarinde et al., 2016; Babarinde and Onyiaocha, 2016; Bhatti et al., 2016; Bilal et al., 2016e; Gangadhara and Prasad, 
2016; Iqbal et al., 2013b; Iqbal, 2016; Iqbal et al., 2015b; Iqbal and Bhatti, 2015b; Iqbal et al., 2016a; Iqbal et al., 2016b; Iqbal and Khera, 2015; Iqbal and Nisar, 2015; Jafarinejad, 2016a; Jamal et al., 2015; Majolagbe et al., 2016a; Mumtaz et al., 2016; Mushtaq et al., 2016; Nadeem et al., 2016b; Nisar et al., 2017; Nisar et al., 2016; Pervaiz et al., 2015; Peter and Chinedu, 2016b; Qureshi et al., 2015a; Rashid et al., 2016; Sayed, 2015; Shindy, 2016; Shoukat et al., 2017; Tahir et al., 2016a; Tahir et al., 2016b; Ukpaka, 2016a; Ukpaka, 2016b; Ukpaka, 2016c, d). For example, mineral salt medium supplemented with as low as $0.02 \%(\mathrm{w} / \mathrm{v})$ yeast extract and glucose was found to remove 70\% ADMI, 69\% COD and $>99 \%$ sorption of heavy metals in $24 \mathrm{~h}$ from the effluent by consortium TSR. Toxicity study of the effluent showed $90 \%$ germination, which was $20 \%-30 \%$ of the untreated effluent for $A$. сера along with other plant species (Patel et al., 2015). Olorunfemi et al. (Olorunfemi et al., 2015c) evaluated bilge water (water from oceanic vessels is usually discharged through the bilge wells) for toxicity using A. cepa test from Nigerian marine environment. Exposure of the onion roots to the wastewater at 1, 5, 10, 25 and $50 \%$ (v/v; wastewater/tap water) showed strong concentrationdependent root growth inhibition with an $\mathrm{EC}_{50}$ value of $52.6 \%$ at $72 \mathrm{~h}$ and induced a variety of chromatid and chromosome structural aberrations at 6, 12, 24 and $48 \mathrm{~h}$. The bilge also disturbed the spindle fibre apparatus at all the concentrations and toxicity was correlated with heavy metals in tested water ( $\mathrm{Cu}, \mathrm{Mn}, \mathrm{Pb}, \mathrm{Fe}, \mathrm{Cd}, \mathrm{Cr}, \mathrm{Ag}, \mathrm{Ni}$ and $\mathrm{Zn}$ ). Mitodepressive and low MI values were also observed. Results revealed toxic chemicals presence in the bilge water, which may contribute to the toxicological assessment of the risk associated with its indiscriminate discharge into the environment of bilge water.

\section{Toxic agents of environmental concern}

While studying the toxicity of waste water samples (mixture of substances) from reserve pit, Vidaković (1993) applied $A$. cepa test. All undiluted waste drilling fluid samples showed cytotoxicity as well as toxicity, which was proved by cytogenetic parameters such as high inhibition of mitotic activity or low MI values and high percentage of chromosomal and genomic aberrations. The toxicity was significantly reduced by increasing the degree of sample dilutions.

Ochratoxin toxicity was evaluated using A. cepa root test. Analyses of root growth and the root meristematic zone in response to Ochratoxin A treatment showed Ochratoxin A toxicity to root growth at a concentration of $10 \mathrm{ug} / \mathrm{mL}$ associated with inhibition of proliferation activity. Cytological changes observed in the Allium chromosome aberrations assay, at a concentration of $5.0 \mathrm{ug} / \mathrm{mL}$ showed that Ochratoxin A was able to induce genotoxicity at the chromosome level. These results indicate that A. cepa is very sensitive to the mycotoxin Ochratoxin A since toxicity and cytogenetic injuries were observed in A. cepa exposed roots (Lerda et al., 2010). The toxicity of glyphosate on A. cepa was also studied (Çavuşoğlu et al., 2011). Germination percentage, root length, seedling weight, MN, CA's and MI end points were used along with root anatomy of $A$. cepa. Glyphosate different doses,100, 250 and $500 \mathrm{mg} / \mathrm{L}$ were applied and results showed significant alterations in the germination percentage, root length, seedling weight, $\mathrm{MN}$, CAs and MI frequency depending on doses of glyphosate. Mitodepressive action on mitosis was also observed and the MI was decreased depending on the dose of appliedglyphosate. Unclear vascular tissue, unclear epidermis layer, cell deformation, unusual form of cell nucleus (usually flat) and binuclear cells were also observed. Each dose of glyphosate caused severe toxic effects on $A$. cepa root cells and the strongest toxic effect was observed at the dose level of $500 / \mathrm{L}$.

The toxic and genotoxic chemical compounds and using A. cepa test, the toxicity and genotoxicity of wastewaters from the public hospital of Buenos Aires (Argentina) was investigated (Magdaleno et al., 2014). The effluent from the sewage treatment plant serving around 10 million inhabitants was also evaluated. The $A$. cepa test showed that $40 \%$ of the samples were genotoxic. In view of genotoxicity, authors suggested the establishment of advanced and effective effluent treatment plants in the hospitals, which are merely dumping the wastewaters in the municipal sewerage system. Similar, the untreated wastewaters from health centers was also evaluated for toxic and genotoxic chemicals. The genotoxicity and toxicity from Hospital San Martín wastewaters was evaluated. This General Hospital releases approximately $560 \mathrm{~m}^{3}$ of effluents daily to the municipal sewer system. The samples collected in summer 2003 revealed presence of toxic and genotoxic agents. However, samples collected in autumn 2004 were non genotoxic to $A$. cepa (Paz et al., 2006). In another study, the $A$. cepa test was used to evaluate the genotoxicity of a hospital effluent in Santa Maria, Rio Grande do Sul State, Brazil (Bagatini et al., 2009). The CA's, anaphasic bridges, and MN during telophase were observed, which indicated the environmental toxicity risk of hospitals wastes. In another study, performance of an Upflow Immobilized Biomass Anaerobic Reactor in treating real hospital wastewater was evaluated using $A$. cepa test. The toxicity was treated and untreated wastewater was assessed by measuring the growth inhibition of $A$. cepa $L$ roots. A. cepa showed reduction in toxicity up to $50 \%$. Authors revealed that the A. cepa was able to verify the toxicity removal capacity of the anaerobic immobilized treatment system (Torres et al., 2014). Kern et al. (2015) also assessed the genotoxicity of hospital laundry wastewaters generated from a regional hospital, Rio Pardo Valley, Rio Grande do Sul, Brazil. A cepa revealed IC 25 value of $51.25 \%$ and cytotoxicity was observed five times the wastewater concentrations. The results warned about the necessity to develop treatment methods to mitigate the environmental impacts caused by the ecotoxicity and genotoxicity of hospital laundry wastewaters. Bernardes et al. (2015) determined toxicity of environmental pollutants i.e., difenoconazole (DZ) and tebuconazole (TZ) on A. cepa by macroscopic (germination and root growth) and microscopic 
(MI, CA, and NA) analyses. A reduction was observed in the germination, root growth, and $\mathrm{MI}$ at higher concentrations of DZ and TZ. CA and NA were also detected in treated roots and DZ and TZ proved to be genotoxic, cytotoxic, and phytotoxic. Wardini et al. (2015) exposed A. cepa roots to compost produced in TPK Sarimukti, Cipatat, West Bandung for toxicity evaluation. Tests carried out by treated $A$. cepa roots with liquid compost $(2,5 \%, 5 \%, 10 \%$ and $12,5 \%(\mathrm{w} / \mathrm{v}))$ or solid compost (25\%, 50\%, 75\% and 100\% (w/v)) for $48 \mathrm{~h}$. Results showed reduced root growth and MI in concentrations dependent manner. $\mathrm{MN}$ increased with increase in liquid compost concentration. $\mathrm{MN}$ found at very high frequencies in highest solid compost concentration $(100 \%)$, but very low at lower concentrations. Cells with binuclei and cell necrosis increased with increasing concentrations of compost. NA found in high frequency in $75 \%$ and $100 \%$ solid compost. Based on results it was concluded that liquid compost is more toxic because it can reduce $\mathrm{MI}$ and root growth rate at lower concentrations than solid compost. Both types of compost have genotoxic properties because it can induce CA, MN, binuclei and NA formation. Hemachandra and Pathiratne (2015) studied the tolerance limits for copper, cadmium and chromium containg industrial effluents into inland surface waters by $A$. серa bioassay. The $A$. cepa roots were exposed to $\mathrm{Cu}(3 \mathrm{mg} \mathrm{L})$ individually or in mixtures with $\mathrm{Cd}(0.1 \mathrm{mg} \mathrm{L})$ or/and $\mathrm{Cr}(0.1$ $\mathrm{mg}$ L) exhibited the highest growth inhibition, MI depression and NA. Root tip cells exposed to $\mathrm{Cr}$ or $\mathrm{Cd}$ alone or in mixture displayed significant CA's. The EC50s for root growth inhibition showed that $\mathrm{Cu}$ was more toxic followed by $\mathrm{Cd}$ and Cr. Authors suggested the industrial effluent discharge regulatory limits for metals to avoid cyto-genotoxicity to biological systems. da Lima et al. (2012) analysed the genotoxic effect of controlled release formulations of the herbicide ametryn encapsulated in microspheres of poly(hydroxybutyrate) and poly(hydroxybutyrate-covalerate) through cytogenetic end points in $A$. серa. The results showed that the rate of chromosome breakdown caused by unencapsulated ametryn was much higher than that caused by ametryn encapsulated in the polymer microspheres, which indicates that controlled release delivery systems employing the polymer formulations should be significantly safer.

Çelik and Aslantürk (2009) investigated the potential genotoxic effects of Ecballium elaterium fruit juice using the $A$. cepa test system. A. cepa were treated with 10 $\mathrm{mL} / \mathrm{L}, 20 \mathrm{~mL} / \mathrm{L}, 50 \mathrm{~mL} / \mathrm{L}$ concentrations and undiluted Ecballium elaterium fruit juice for $72 \mathrm{~h}$ and dose-dependent inhibition of root growth and mitodepressive effects on cell division in A. cepa root tip cells were observed. Among CA's, breaks, stickiness and pole deviations as well as $\mathrm{MN}$ inductions were also observed. Bratu et al. (2012) measured the phytotoxicity/toxicity of aqueous solutions of powder extract using A. cepa as a test organism. Aqueous solutions of the fruit powder have reasonably expressed mutagenic activity in vivo on the radicles of $A$. серa, especially when they are used at a concentration of $1 \mathrm{~g} / \mathrm{dL}$ for a prolonged time $(48 \mathrm{~h})$. At lower concentrations $(0.1 \mathrm{~g} / \mathrm{dL})$, however, the mutagenic effect was not observed. On the basis of results, authors suggested the use of Sambucus nigra fruit extract powder at low concentrations for possible applications in the food industry. Hile studying the toxicity of irrigation water used for vegetables in a Greater São Paulo watershed region, A. cepa test was applied (de Maio Lacerda et al., 2014). The samples were collected from small watercourses located in a sub-basin of High Tietê, Cotia-Guarapiranga, Embu-Mirim River, a major tributary of the Guarapiranga Reservoir. From April to September 2011, water and sediment samples were collected at three points (small watercourses) used for irrigation of leafy vegetables inside private agricultural properties (April, June and September). Surface water genotoxic effect was detected in 2 out of the 3 points and mutagenic effect in all three sampled points, as well as in the sediment.

The cytotoxicity and the mutagenicity of liquid waste produced in the process of industrialization of the bitter cassava (a plant used as food and an ingredient in industry), olho-junto variety was also studied using A. cepa test (Viana et al., 2014). The cassava root contains wastewater, popularly known as manipueira, which is a toxic substance. Its ingestion by animals causes poisoning although they react positively to treatment with sodium thiosulfate. The liquid waste was obtained when the cassava roots are pressed; pond water, which is press water stored in impounded ponds; and a solution of sodium thiosulfate, pure and with other waste. The toxicity was evaluated by root $A$. cepa meristematic cells. Treatment with saline solution showed cytotoxicity in $A$. cepa and reduced cell division significantly. Clastogenic effect was also observed through CA test. Based on results, authors suggested the use of sodium thiosulfate only in emergency conditions in which the benefits exceed the risks.

While studying the food industry ingredient used as flavoring agent, Moura et al. (2016) employed A. cepa assay for toxicity monitoring of synthetic Cheese and Cheddar Cheese (food flavors) at 1.0 and $2.0 \mathrm{~mL}$ doses. Genotoxiicty was not observed, however, flavoring agents significantly reduced the cell division rate. Adeyemo and Farinmade (2016) studied genotoxic and cytotoxic effects of monosodium glutamate (MSG) used as flavor enhancer in foods using the $A$. cepa test. A. cepa roots were exposed to 1 , 3, 5 and $7 \mathrm{~g} / \mathrm{L}$ of MSG concentrations and subjected to macroscopic (morphology and color of roots) and microscopic (MI and CA's) analysis. MSG inhibited growth of $A$. cepa roots at all tested concentrations. MSG also reduced the number of roots growing from primodium in all tested concentrations and the least was observed in $5 \mathrm{~g}$ and $7 \mathrm{~g} / \mathrm{L}$ concentration. Color of root tips range from brownish to dark brown or black in higher MSG concentrations was observed. Sticky chromosomal aberration at telophase was most observed at all the MSG concentrations. MSG also decreased MI values slightly. 


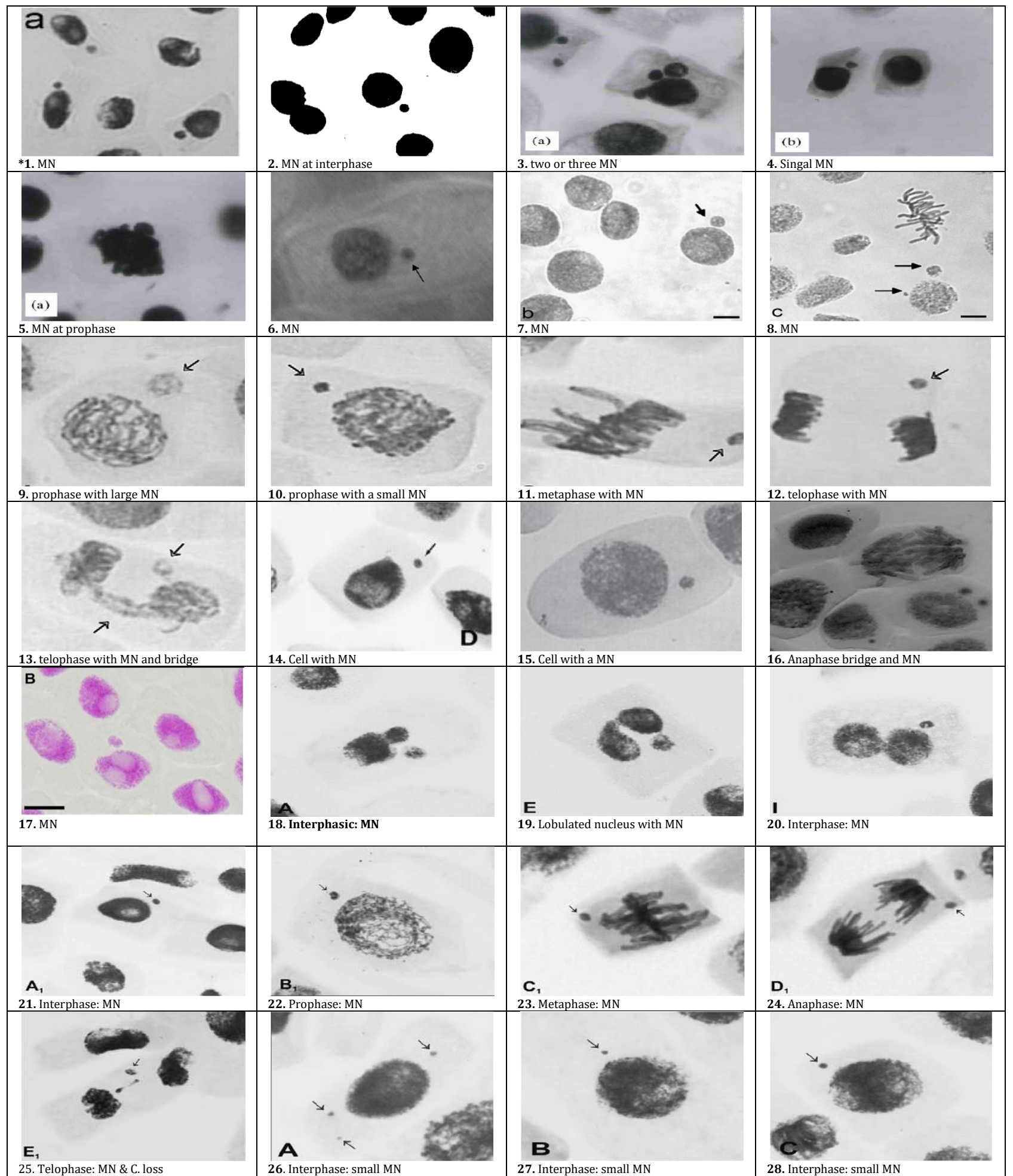

Fig. 4: A. сера meristematic cells carrying micronuclei and their types as a result of different mutagenic agent exposure (1-40). *References and physiochemical properties of wastes: 1 (Bianchi et al., 2011): river water mixed with industrial effluents: pH-6.83-7.29, EC-29-114, D0-4.43-7.09 mg/L, T-24.1-25.9 ${ }^{\circ} \mathrm{C}$ and metal (Cu, Cr, Ni, Cd, Zn, Fe, Mn, Ca). 2 (El-Shahaby et al., 2003): industrial wastewater. 3-4 (Abdel Migid et al., 2007): fertilizer industrial effluent before algal treatment. 5 (Abdel Migid et al., 2007): industrial effluent before algal treatment. 6 (Gupta and Ahmad, 2011): 7-8 (Kwasniewska et al., 2012): landfill leachates: $\mathrm{Pb}, \mathrm{Ni}, \mathrm{Cd}, \mathrm{Hg}$, benzene, dichloromethane, chloroform, trichlorobenzenes, exachlorobenzene, hexachlorobutadiene, hexachlorocyclohexane, lindane, hexachlorocyclohexane, pentachlorobenzene, pentachlorophenol, PAH's. 9-13 (Leme and Marin-Morales, 2008): refinery wastewater: $\mathrm{HC}_{2}\left(\mathrm{C}_{12}-\mathrm{C}_{18}\right)$ and PAH's (naphthalene, acenaphthylene, acenaphthlene, fluorine, phenanthrene, pyrene, benzoanthracence and chrysene). 14 (Mazzeo et al., 2011): mixture of benzene, toluene, ethylbenzene and xylene. 15 (Ventura-Camargo et al., 2011): azo dye. 16 (Jadhav et al., 2010): textile effluent. 17 (Herrero et al., 2012 ): Di(2ethylhexyl)phthalate, triclosan and propylparaben. 18-33 (Leme and Marin-Morales, 2009). 34 (Olorunfemi et al., 2011): A. cepa grown in agriculture filed under the effect of pesticides. 35-36 (Ivanova et al., 2005): heavy metal and cyanide contaminated river waters. 37-38 (Ivanova et al., 2008). 39-40 (Staykova et al., 2005). 


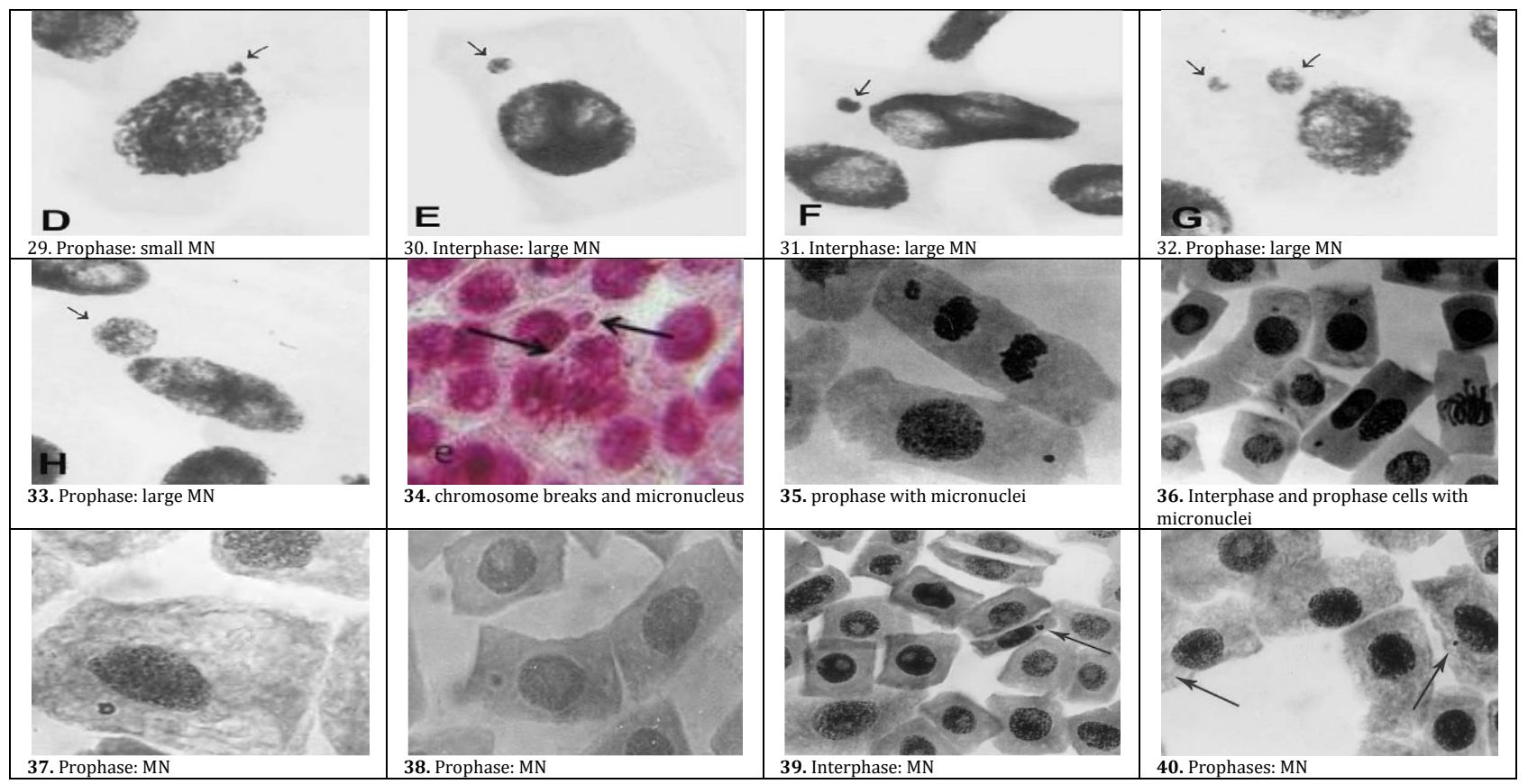

Fig. 4: Continue...

In order to evaluate the cytogenetic effects of food preservatives (sodium benzoate and sodium metabisulphite) Onyemaobi et al. (2011) employed $A$. cepa assay considering root length, CA and MI end points. The MI decreased with increasing concentration of both sodium benzoate and sodium metabisulphite. Cytological aberrations observed were clumping, fragmentation, pulverization, lagging, binucleate cells and reduction in chromatin materials. The percentage of CA's increased with increase in concentration of the food preservatives. The effects of sodium metabisulphite at different concentrations were very detrimental as more aberrations were recorded even after the recovery experiment. Authors suggested the banning of these substances as food preservatives since irreversible cytotoxic effects at certain doses were observed. Tripathy et al. (2015) studied the toxicity of dye orange red (amalgamation of two primary food colourants like caramoisine and sunset yellow). Assuming the potential genotoxicity of the dye, A. cepa mitotic aberrations end point and gradual decondensation or little condensation of chromosomal arms in abnormal metaphases and anaphases were observed. Unequal cytokinesis and karyokinesis and formation of bi-nucleated cells were also appeared. The frequencies of MA showed good correlation with the concentration of dye.

In view of use of nano materials for food packing, De Lima et al. (2010) proposed that such nanoparticles might harm food quality and tested the chitosan (CS) and poly(methacrylic acid) (PMAA) nanoparticles (size 60, 82, and $111 \mathrm{~nm}$ ) for genotoxicity using the A. cepa cytogenetic tests. Test substrates were exposed to solutions containing nanoparticles at polymer mass concentrations of $1.8,18$, and $180 \mathrm{mg} / \mathrm{L}$ and particles of 82 and $111 \mathrm{~nm}$ nanoparticles reduced MI values at the highest concentration tested (180 $\mathrm{mg} / \mathrm{L}$ ), indicating that the nanoparticles were toxic. In the case of the $60 \mathrm{~nm} \mathrm{CS} / \mathrm{PMAA}$ nanoparticles, no significant changes in the MI was observed at the concentration levels tested, indicating that these particles were not toxic. The techniques used show promising potential for application in tests of nanoparticle safety envisaging the future use of these materials in food packaging. Lutterbeck et al. (2015) evaluated the toxic effects of four frequently used anti-cancer drugs (Cyclophosphamide (CP), Methotrexate (MTX), 5Fluorouracil (5-FU) and Imatinib (IM). Cytotoxicity, genotoxicity and mutagenicity investigations were performed with $A$. cepa assays. Significant differences in the MI were observed in three of the studied compounds (MTX, 5-FU and CP), indicating potential cytotoxic activity of these substances. CA's were registered in cells that were exposed to 5-FU, CP and IM. All the four compounds caused the formation of micronucleated cells indicating mutagenic potential. Besides, the assays performed with MTX samples presented a high number of cell apoptosis (cell death). So, authors suggested the use of $A$. серa for the screening of environmental contamination.

Sobral et al. (2013) studied the toxicity of metal rich acid mine drainage (AMD) using CA end point. Root was exposed to 100, 10, 1, and $0.1(\%)$ of AMD. Chromosomal aberrations, cell division phases and cell death were quantified after 24 and $48 \mathrm{~h}$ recovery periods. The AMD revealed to be mutagenic and genotoxic, even after diluting it to 1 and $0.1 \%$. Recovery tests showed that AMD genotoxic effects persisted after the exposure. Netto et al. (2013) also evaluated that the carboniferous activity generates acid mine drainage (AMD) which is capable of unleashing toxic effects on the exposed biota. The untreated-AMD and AMD treated with calcinated sediment was tested for toxicity and it was revealed that untreated-AMD presented low $\mathrm{pH}$ values and 
elevated concentrations of the metals $\mathrm{Fe}, \mathrm{Al}, \mathrm{Mn}, \mathrm{Zn}$ and $\mathrm{Cu}$. $\mathrm{A}$. cepa roots showed sub-chronic toxicity and genotoxicity and DNA exposed to untreated-AMD. Treatment of AMD with calcinated sediment reduced the acidity and metals, which in trun showed less toxic and genotoxic effects. Similarly, Defaveri et al. (2009) evaluated the efficiency of physical, chemical, and biological treatment of acid mine drainage using alternative biological indicator A. cepa. Samples were collected at four stations that are located at specific treatment system: 1) $\mathrm{pH}$ control and precipitation, 2) biological damping pond outlet, 3) wetland inlet, and 4) wetland outlet. Toxicity in A. cepa was observed as root growth inhibition after a seven-day exposure period at 100\% effluent concentration and in comparison to shrimp and comet assay, results revealed that A. cepa is only suitable for evaluating genotoxicity and in evaluating treatment process efficiency in eliminating toxicity and genotoxicity. Geremias et al. (2012) evaluated the efficacy of the treatment of acid mine drainage (AMD) with calcinated coal mining waste using A. cepa. The $\mathrm{pH}$ values and the concentrations of aluminum, iron, manganese, zinc, copper, lead and sulfate were determined before and after the treatment of the AMD with calcinated coal mining waste. A. cepa roots were exposed to untreated and treated AMD. The results indicated that the AMD treatment with calcinated coal mining waste resulted in an increase in the $\mathrm{pH}$ and an expressive removal of aluminum, iron, manganese and zinc. A high sub-chronic toxicity was observed when A. cepa was exposed to the untreated AMD. However, after the treatment no toxicity was detected. In conclusion, the use of calcinated coal mining waste associated with toxicological tests on $A$. cepa represents an alternative system for the treatment and biomonitoring of these types of environmental contaminants. Tabrez and Ahmad (2012) analysed Trichloroethylene (TCE) genotoxic, which is usually present in the air, soil and water as pollutant. For the genotoxicity analysis of TCE in natural milieu, wastewater collected from two different stations of northern India namely Saharanpur and Aligarh, U.P were supplemented with 50 and $100 \mathrm{mg} / \mathrm{L}$ of trichloroethylene. TCE alone was found to be nongenotoxic up to the range of $1000 \mathrm{mg} / \mathrm{L}$ concentration. However, the test water samples supplemented with 100 $\mathrm{mg} / \mathrm{L}$ of TCE, exhibited a significant increase in the genotoxicity. A. серa genotoxicity test also showed around $25 \%$ increase in CA's frequencies following $100 \mathrm{mg} / \mathrm{L} \mathrm{TCE}$ supplementation. However, supplementation of $50 \mathrm{mg} / \mathrm{L}$ TCE to the test water samples could not enhance the genotoxicity to a significant extent. From results, it can be concluded that TCE itself was non-genotoxic, but it may be toxic at a concentration of $100 \mathrm{mg} / \mathrm{L}$ under certain environmental conditions. Yahaya et al. (2012) monitored the cytotoxicity and mutagenicity of cement dust. A. cepa roots were exposed to cement dust over three different periods of time at about $100 \mathrm{~m}$ from a cement factory. The test groups (groups 2-4) were exposed to the dust for 2 weeks, 4 weeks and 6 weeks, respectively. The elemental analysis of the A. cepa in the test groups revealed significant levels of calcium, silicon, aluminum, chromium and lead compared to the control group. Also, significant differences were found to exist among the levels of the elements detected in the A. cepa in various test groups. There was a direct linear relationship between the concentrations of calcium and root length growths of the A. cepa across the groups. The CA's such as stickiness, cmitosis, chromosomal bridge, chromosome fragmentation, vagrant chromosomes, bi-nucleus chromosomes and multipolar anaphase were observed, which increased with the length of exposure. The findings of the research highlight the toxicity of cement dust and the need for pollution control measures to safeguard plants and animals in the environment. Similarly, Aguiar et al. (2016) studied the toxicity of industry treating stones i.e., marbles and granites, includes a stage of plate polishing, in which resins and abrasives are used, producing a fine grained waste with high moisture content. The A. cepa bulbs were germinated in Petri dishes with filter paper moistened in the liquid phase of the effluent and germinated roots were prepared for analysis of CA's. The MI values were same in all treatments, however, seeds exposed to the polishing waste showed high MN induction, nuclear buds and other CA's. Authors concluded that the analysed wastes have mutagenic potential that is correlated with high content of phenolic compounds identified in the samples.

Chakraborty et al. (2009) tested fly ash (by-product of coal-fired electricity generation plants) for toxicity using A. cepa assay from thermal power plant in West Bengal, India. Fly ash sample was mixed with sand in different proportions and A. cepa bulbs were germinated in fly ash for five days. The results indicated that fly ash at $100 \%$ concentration inhibitted root growth and mitotic indices; induced binucleated cells as a function of the proportion, but was not toxic at very low concentration. The metals like $\mathrm{Zn}, \mathrm{Pb}, \mathrm{Cu}, \mathrm{Ni}$, $\mathrm{Cd}$ and As were present in fly ash sample and partially correlated with fly ash toxicity. Authors suggested the use of fly ash at very low concentrations in order to protect the ecosystem from any potential adverse effects. Maiti et al. (2016) studied the toxicity of hair dye and estimated it's discharge to the environment. Recommended dose precisely mentioned in instructions booklet of the hair dye was used to expose A. cepa roots along with other bioassays. A. cepa MN assay showed various CA's along with cell division retardation. Including A. cepa results, all bioassays confirmed cytotoxicity and mutagenicity of hair dyes on living cells.

Roychoudhury and Giri (1989) reported the effects of 4 permitted food dyes, i.e., fast green FCF, indigo carmine, orange $\mathrm{G}$ and tartrazine, and the non-permitted dye metanil yellow on chromosomes of A. cepa. A significant increase in polyploid cells was observed in all cases. High doses of dyes induced chromosome breaks and MN formation. Although all dyes produced mitotic aberrations, metanil yellow and fast green FCF showed comparatively stronger clastogenic activity.

Blagojević et al. (2009) evaluated melted snow using A cepa anaphase-telophase test from Belgrade, the capital city of Serbia. Falling snow collects pollutants from the air 
and could cause genotoxicity. Snow samples were collected from two sites, characterized by differences in pollution intensity. At the more polluted site the analyses showed a very high degree of both toxicity and genotoxicity in the first year of the study. At the other site the situation was much better but not without warning signals. The results showed that standard analyses for the presence of certain contaminants in the air do not give an accurate picture of the possible consequences of urban air pollution because the genotoxic potential remains hidden. The $A$. cepa test has been demonstrated to be very convenient for evaluation of air pollution through analyses of melted snow samples.

Glasencnik et al. (2002) assessed the air pollution on the genetic material of $A$. cepa cytogenetic analysis. Roots were exposed at eight sampling plots in the vicinity of major Slovene local emission sources in 1999 and CA's were measured at each sampling plot. No significant differences in the frequency of CA were found among localities in the clay pot experiment. However, under field experiments the significantly differed CA frequencies were observed among sampling plots. Results confirmed that genotoxic agent in air were present. Similarly, Glasenčnik et al. (2004) later expoused $A$. cepa to field conditions at six research plots in the most polluted areas, Slovenia in the vegetation seasons in 1999 and 2000 and MI and CA end points were analysed. Significant differences in the MU and CA's at different sampling plots were observed and the correlation between the ozone concentration and the MI was determined. Based on results, the selected sites were decleared polluted.

Tkalec et al. (2009) measured the effects of exposure to radiofrequency electromagnetic fields (RF-EMFs) on seed germination, primary root growth as well as mitotic activity and mitotic aberrations in root meristematic cells of $A$. cepa. Seeds were exposed for $2 \mathrm{~h}$ to EMFs of 400 and $900 \mathrm{MHz}$ at field strengths of $10,23,41$ and $120 \mathrm{Vm}^{-1}$. The effect was also measured for longer exposure time $(4 \mathrm{~h})$ and field modulation $23 \mathrm{Vm}^{-1}$. Germination rate and root length did not change significantly after exposure to radiofrequency fields under any of the treatment conditions. At $900 \mathrm{MHz}$, exposures to EMFs of higher field strengths (41 and $120 \mathrm{~V} \mathrm{~m}^{-1}$ ) or to modulated fields showed a significant increase of the MI value, while the percentage of mitotic abnormalities increased after all exposure treatments. On the other hand, at $400 \mathrm{MHz}$ the MI increased only after exposure to modulated EMF. At this frequency, higher numbers of mitotic abnormalities were found after exposure to modulated EMF as well as after exposure to EMFs of higher strengths (41 and $120 \mathrm{~V} \mathrm{~m}^{-1}$ ). The types of aberration induced by the EMFs of both frequencies were quite similar, mainly consisting of lagging chromosomes, vagrants, disturbed anaphases and chromosome stickiness. Results showed that non-thermal exposure to the radiofrequency fields can induce mitotic aberrations field frequencies dependent manner as well as field strength and modulation.

Olorunfemi et al. (2012) studied the cytotoxic and genotoxic effects of ballast water using A. cepa. A. cepa roots were treated with different concentrations $(0.5 \%, 1 \%, 5 \%$, and $10 \%$ ) of ballast water for $48 \mathrm{~h}$ and analysed for cytological effects. All concentrations induced CA's in the root tip cells along with MI reduction in increasing concentration manner and root growth inhibition with an $\mathrm{EC}_{50}$ value of $15 \%$ after $72 \mathrm{~h}$ was recorded. Authors highlighted that lack of enforced regulations on the exchange of ballast water from oceangoing ships can led to indiscriminate discharge of toxic agent in the environment. Alos (Olorunfemi et al., 2014b) reported DNA damage in $A$. cepa by exposing roots to ballast water at $0.5,1,5$ and $10 \%$ concentrations.

Singh et al. (2014) studied the genotoxic effects of surface water from five sites of Hooghly River in West Bengal, India, along the banks of which many shipbuilding and scrap industries are located using $\mathrm{MN}$ and CA's end points in $A$. сера. A. сера exposed roots showed morphological distortions, MN inductions and CA's. The MI was lower than $50 \%$ in the treated samples and authors concluded that the workers of local shipbuilding and scrap industries, the residents of nearby areas and the aquatic biodiversity are vulnerable to health hazardous if exposed to contaminated water.

In view of application of pyrolysis for biomass conversion to oil, Holan et al. (2014) studied the pyrolysis of oils produced from different sources (poplar, beech and spruce) for any toxic and/or genotoxic effects using $A$. серa assay. The oils were diluted with water to gain different concentrations and water-soluble fractions of the oils were tested for toxicity. The macroscopic and microscopic analysis of roots, exposed to different concentrations affectd the roots (shape and root length) depending on oil concentrations as well as the MI values were also found variable among treatment. Other abnormalities i.e., damaged dividing cells were in following order poplar-oil > beech-oil and so on. The results indicate that all the three pyrolysis oils exert a toxic, cytotoxic and genotoxic effect. The differences in toxicity and genotoxicity between the oils are probably due to variations in chemical composition, as a result of the different biomass feedstocks used in the pyrolysis oils. A. cepa test proved to be a sensitive indicator of toxicity and genotoxicity.

Silva (Silva, 2014) evaluated the toxicity and genotoxicity of different sizes of silica nanoparticles (SiNP) using A. cepa along with other bioassays. Specimen were exposed to TM40 (22 nm), HS30 (12 nm), SM30 (7 nm) with concentrations ranging from 0.19 to $163.8 \mathrm{~g} / \mathrm{L}$ (TM40) and 0.29 to $122.85 \mathrm{~g} / \mathrm{L}$ (HS30 and SM30), and tested for germination rate, growth and DNA damage. Within each test SiNP present a size dependent chronic toxicity. All particles revealed phytotoxicity in $A$. серa along with genotoxicity. Author revealed that SiNP size can strongly influence toxicity.

Electronic waste is very common in the environment, and was also investigated for cytogenic effect on $A$. cepa from Iloabuchi electronic market, Diobu, Rivers State, Nigeria (Babatunde and Anabuike, 2015). Roots exposed to concentrations of 5\%,10\%, 25\%, 50\%, and $100 \%$ showed root growth inhibition at all concentrations in concentration dependent manner. Various morphological defects of the onion roots were observed including short, 
crochet roots, C-tumor roots and severe toxic effects. In the in vivo genotoxity assay, all samples lowered the frequency of mitotic cells in concentration dependent along with significant induction of aberrations cells, which were correlated with higher metal concentration in e-waste leachate.

\section{CONCLUSIONS}

From literature survey, it is concluded that the watersheds and soil contaminated with industrial wastewater have cytotoxic, genotoxic and mutagenic properties. All kinds of wastes, wastewater, and soils irrigated with wastewater and contaminated river water showed severe effects on genetic material of $A$. cepa from root inhibition to DNA damage. However, chromosomal aberrations were found most common.. Furthermore, $A$. cepa test was found to be sensitive and efficient to detect the toxic agents present in industrial effluents and other contaminated bodies. Thus, A. cepa can be used for evaluation of toxicity of industrial wastes as well as other adjoining territories and their results can be used as a warning for biological and ecological systems. Furthermore, there is need to standardize the $A$. cepa test, because factors such as solvent extraction, sampling season, $\mathrm{pH}$, temperature and exposure of $A$. cepa seed to background pollutants may affect the sensitivity of this test. Because every sphere of life is contaminated and the establishment of international centers of stock seed for the production and supply of unexposed seed for experimentation may be useful and results, thus obtained can be used as biomarker to identify toxicological status of the industrial wastewater with assurance of authenticity.

\section{REFERENCES}

Abbas, W., Bokhari, T.H., Bhatti, I.A., Iqbal, M., 2015. Degradation Study of Disperse Red F3BS by Gamma Radiation $/ \mathrm{H}_{2} \mathrm{O}_{2}$. Asian Journal of Chemistry 27, 282-286.

Abbasi, U., Jin, W., Pervez, A., Bhatti, Z.A., Tariq, M., Shaheen, S., Iqbal, A., Mahmood, Q., 2016. Anaerobic microbial fuel cell treating combined industrial wastewater: Correlation of electricity generation with pollutants. Bioresource technology 200, 1-7.

Abdel-Azeem, E.A., 2014. The detrimental effects and mutagenical potential of Schiff base sulfadiazine derivative engineered particles on Allium cepa (L). Report and Opinion 6, 48-58.

Abdel Migid, H.M., Azab, Y.A., Ibrahim, W.M., 2007. Use of plant genotoxicity bioassay for the evaluation of efficiency of algal biofilters in bioremediation of toxic industrial effluent. Ecotoxicology and environmental safety 66, 57-64.

Abu, N.E., Asomba, C., Ubani, C., 2015. Chemical composition and mutagenic assessment of petrochemical effluents on onion (Allium cepa) root tip mitosis. African Journal of Biotechnology 14, 2740-2747.
Abu, N.E., Ezeugwu, S.C., 2008. Risk evaluation of industrial wastewater on plants using onion (Allium cepa l.) chromosome aberration assay. Journal of Tropical Agriculture, Food, Environment and Extension 7, 242 248.

Abu, N.E., Mba, K., 2011. Mutagenecity testing of phamarceutical effluents on Allium cepa root tip meristems. Journal of Toxicology and Environmental Health Sciences 3, 44-51.

Adeel, S., Gulzar, T., Azeem, M., Saeed, M., Hanif, I., Iqbal, N., 2017. Appraisal of marigold flower based lutein as natural colourant for textile dyeing under the influence of gamma radiations. Radiation Physics and Chemistry 130, 35-39.

Adeel, S., Usman, M., Haider, W., Saeed, M., Muneer, M., Ali, M., 2015. Dyeing of gamma irradiated cotton using Direct Yellow 12 and Direct Yellow 27: improvement in colour strength and fastness properties. Cellulose 22, 20952105.

Adegbite, A., Sanyaolu, E., 2009. Cytotoxicity testing of aqueous extract of bitter leaf (Vernonia amygdalina Del.) using the Allium cepa chromosome aberration assay. Scientific Research and Essays 4, 1311-1314.

Adelanwa, M., Osazee, O., Adelanwa, E., 2011. Screening of cytotoxicity and genotoxicity in selected boreholes water by the use of Allium cepaassay in Benin Metropolis, Nigeria. Journal of Environmental Issues and Agriculture in Developing Countries 3, 149.

Adesola, B., Ogundipe, K., Sangosanya, K.T., Akintola, B.D., Oluwa, A., Hassan, E., 2016. Comparative study on the biosorption of $\mathrm{Pb}(\mathrm{II}), \mathrm{Cd}(\mathrm{II})$ and $\mathrm{Zn}$ (II) using Lemon grass (Cymbopogon citratus): Kinetics, isotherms and thermodynamics. . Chemistry International 2, 89-102.

Adeyemo, O.A., Farinmade, A.E., 2016. Genotoxic and cytotoxic effects of food flavor enhancer, monosodium glutamate (MSG) using Allium cepa assay. African Journal of Biotechnology 12.

Aguiar, L.L., Tonon, C.B., Nunes, E.T., Braga, A.C.A., Neves, M.A., de Oliveira David, J.A., 2016. Mutagenic potential of fine wastes from dimension stone industry. Ecotoxicology and Environmental Safety 125, 116-120.

Ahmed, F.A.W., 2014. Cytotoxic and Genotoxic Potency Screening of WIDE-SPEC Pesticide on Allium cepa L. Root Meristem Cells. Journal of Natural Sciences Research 4, 100-108.

Akinbola, T.I., Adeyemi, A., Morenikeji, O.A., Bakare, A.A., Alimba, C.G., 2011. Hospital waste incinerator bottom ash leachate induced cyto-genotoxicity in Allium cepa and reproductive toxicity in mice. Toxicology and Industrial Health 27, 505-514.

Akinboro, A., Mohammed, K., Rathnasamy, S., Muniandy, V.R., 2011. Genotoxicity Assessment of Water Samples from the Sungai Dua River in Pulau Pinang, Malaysia, Using the Allium cepa Test. Tropical Life Sciences Research 22, 2335.

Akinsemolu, A.A., Nwangburuka, C.C., Ogunwenmo, K.O., 2015. Evaluation of Tobacco Industrial Wastewater for 
Genotoxic Characteristics on Allium cepa L. Root Cell Mitosis. Journal of Advances in Biology and Biotechnology 2, 165-173.

Akintonwa, A., Awodele, O., Olofinnade, A., Anyakora, C., Afolayan, G., Coker, H., 2009. Assessment of the mutagenicity of some pharmaceutical effluents. American Journal of Pharmacology and Toxicology 4, 144-150.

Al, G., Özdemir, U., Aksoy, Ö., 2013. Cytotoxic effects of Reactive Blue 33 on Allium cepa determined using Taguchi's L8 orthogonal array. Ecotoxicology and Environmental Safety 98, 36-40.

Albertini, R.J., Anderson, D., Douglas, G.R., Hagmar, L., Hemminki, K., Merlo, F., Natarajan, A., Norppa, H., Shuker, D.E.G., Tice, R., 2000. IPCS guidelines for the monitoring of genotoxic effects of carcinogens in humans. Mutation Research/Reviews in Mutation Research 463, 111-172.

Alberts, B., Johnson, A., Lewis, J., Raff, M., Roberts, K., Walter, P., Bray, D., Watson, J., 2008. Molecular Biology of the Cell (New York: Garland Science, 2008). Classic Textbook, $5^{\text {th }}$ Edition.

Ali, Ö., 2010. Cytotoxicity of Hibiscus rosa-sinensis flower extract. Caryologia 63, 157-161.

Alimba, C.G., Ogunkanmi, A.L., Ogunmola, F.J., 2013. Cytotoxic and genotoxic assessment of textile effluent using Allium assay. Toxicology 9, 45.

Amadi, S., Ukpaka, C., 2015. Role of molecular diffusion in the recovery of water flood residual oil. Chemistry International 2, 103-114.

Ameta, S., Punjabi, P., Kothari, S., Sancheti, A., 2003. Effect of Untreated and Photocatalytically Treated Dyeing Industry Effluent on Growth and Biochemical Parameters of Allium cepa (Onion). Pollution Research 22, 389-392.

Amin, A.W., 2002. Cytotoxicity Testing of Sewage Water Treatment Using Allium cepa Chromosome Aberrations Assay. Pakistan Journal of Biological Sciences 5, 184-188.

Andrade-Vieira, L.F., de Campos, J.M.S., Davide, L.C., 2012. Effects of Spent Pot Liner on mitotic activity and nuclear DNA content in meristematic cells of Allium cepa. Journal of Environmental Management 107, 140-146.

Andrade, L., Campos, J., Davide, L., 2008. Cytogenetic alterations induced by SPL (spent potliners) in meristematic cells of plant bioassays. Ecotoxicology and Environmental Safety 71, 706-710.

Andrioli, N., Wulff, A., Mudry, M., 2006. Allium cepa como biomonitor de toxicidad y genotoxicidad de metronidazol. Theoría 15, 9-16p.

Arambašić, M.B., Bjelić, S., Subakov, G., 1995. Acute toxicity of heavy metals (copper, lead, zinc), phenol and sodium on Allium cepa L., Lepidium sativum L. and Daphnia magna St.: Comparative investigations and the practical applications. Water Research 29, 497-503.

Arkhipchuk, V., Goncharuk, V., Chernykh, V., Maloshtan, L., Gritsenko, I., 2004. Use of a complex approach for assessment of metamizole sodium and acetylsalicylic acid toxicity, genotoxicity and cytotoxicity. Journal of Applied Toxicology 24, 401-407.
Arya, S., Mukherjee, A., 2014. Sensitivity of Allium cepa and Vicia faba towards cadmium toxicity. Journal of Soil Science and Plant Nutrition 14, 447-458.

Asadi, A., Zinatizadeh, A.A., Van Loosdrecht, M., 2016. A novel continuous feed and intermittent discharge airlift bioreactor (CFIDAB) for enhanced simultaneous removal of carbon and nutrients from soft drink industrial wastewater. Chemical Engineering Journal 292, 13-27.

Asgher, M., Iqbal, H.M.N., 2013. Enhanced catalytic features of sol-gel immobilized MnP isolated from solid state culture of Pleurotus ostreatus IBL-02. Chinese Chemical Letters 24, 344-346.

Ashar, A., Iqbal, M., Bhatti, I.A., Ahmad, M.Z., Qureshi, K., Nisar, J., Bukhari, I.H., 2016. Synthesis, characterization and photocatalytic activity of $\mathrm{ZnO}$ flower and pseudo-sphere: Nonylphenol ethoxylate degradation under UV and solar irradiation. Journal of Alloys and Compounds 678, 126136.

Asita, A.O., Makhalemele, R.e., 2008. Genotoxicity of Chlorpyrifos, Alpha-thrin, Efekto virikop and Springbok to onion root tip cells. African Journal of Biotechnology 7.

Asita, A.O., Matobole, R., 2010. Comparative study of the sensitivities of onion and broad bean root tip meristematic cells to genotoxins. African Journal of Biotechnology 9, 4465-4470.

Asita, O.A., Makhalemele, R., 2009. Genotoxic effects of dithane, malathion and garden ripcord on onion root tip cells. African Journal of Food, Agriculture, Nutrition and Development 9.

Așkin Çelik, T., Aslantürk, Ö.S., 2010. Evaluation of cytotoxicity and genotoxicity of Inula viscosa leaf extracts with Allium test. Journal of BioMed Research 2010.

Aslantürk, Ö.S., Çelik, T.A., 2006. Protective effect of lycopene on ethyl methane sulfonate induced chromosome aberrations in Allium cepa. Caryologia 59, 220-225.

Ateeq, B., Abul Farah, M., Niamat Ali, M., Ahmad, W., 2002. Clastogenicity of pentachlorophenol, 2,4-D and butachlor evaluated by Allium root tip test. Mutation Research/Genetic Toxicology and Environmental Mutagenesis 514, 105-113.

Athanásio, C.G., Prá, D., Rieger, A., 2014. Water Quality of Urban Streams: The Allium cepa Seeds/Seedlings Test as a Tool for Surface Water Monitoring. The Scientific World Journal 2014.

Babarinde, A., Ogundipe, K., Sangosanya, K.T., Akintola, B.D., Elizabeth Hassan, A.-O., 2016. Comparative study on the biosorption of $\mathrm{Pb}(\mathrm{II}), \mathrm{Cd}(\mathrm{II})$ and $\mathrm{Zn}(\mathrm{II})$ using Lemon grass (Cymbopogon citratus): kinetics, isotherms and thermodynamics. Chemistry International 2, 89-102.

Babarinde, A., Onyiaocha, G.O., 2016. Equilibrium sorption of divalent metal ions onto groundnut (Arachis hypogaea) shell: kinetics, isotherm and thermodynamics. Chemistry International 2, 37-46.

Babatunde, B., Anabuike, F., 2015. In Vivo Cytogenotoxicity of Electronic Waste Leachate from Iloabuchi Electronic 
Market, Diobu, Rivers State, Nigeria on Allium Cepa. Challenges 6, 173-187.

Babu, K., Deepa, M., Shankar, S.G., Rai, S., 2008. Effect of nanosilver on cell division and mitotic chromosomes: a prefatory siren. Internetional Journal of Nanotechnology $2,2$.

Bagatini, M., Vasconcelos, T., Laughinghouse IV, H., Martins, A., Tedesco, S., 2009. Biomonitoring hospital effluents by the Allium cepa L. test. Bulletin of Environmental Contamination and Toxicology 82, 590-592.

Bakare, A.A., 2001. The potential mutagenic effect of the leachates of rural solid waste landfill on Allium cepa (L.). SINET: Ethiopian Journal of Science 24, 283-291.

Bakare, A.A., Ademeso, M., Adetunji, A., Alabi, A., 2011. Pharmaceutical effluent induced chromosome aberration in rat bone marrow cells. Archives of Applied Science Research 3, 345-352.

Bakare, A.A., Adeyemi, A.O., Adeyemi, A., Alabi, O.A., Osibanjo, 0., 2012. Cytogenotoxic effects of electronic waste leachate in Allium cepa. Caryologia 65, 94-100.

Bakare, A.A., Okunola, A.A., Adetunji, O.A., Jenmi, H.B., 2009. Genotoxicity assessment of a pharmaceutical effluent using four bioassays. Genetics and Molecular Biology 32, 373-381.

Balan, K., Pratheebaa, P., Palvannan, T., 2013. Application of the laccase, produced on coconut flesh by Pleurotus florida for dye decolorization. Journal of Water Chemistry and Technology 35, 273-280.

Balan, K., Sathishkumar, P., Palvannan, T., 2012. Decolorization of malachite green by laccase: Optimization by response surface methodology. Journal of the Taiwan Institute of Chemical Engineers 43, 776782.

Balapure, K., Jain, K., Bhatt, N., Madamwar, D., 2016. Exploring bioremediation strategies to enhance the mineralization of textile industrial wastewater through sequential anaerobic-microaerophilic process. International Biodeterioration \& Biodegradation 106, 97 105.

Bandyopadhyay, A., 2015. Cytogenetic Responses of Plant Root Meristematic Cells to the Arsenic (III) Contaminated Groundwater in Eastern Parts of Burdwan District, WB, India. International Journal of Plant and Environment 1, 15.

Barberio, A., Barros, L., Voltolini, J., Mello, M., 2009. Evaluation of the cytotoxic and genotoxic potential of water from the River ParaÃba do Sul, in Brazil, with the Allium cepa L. test. Brazilian Journal of Biology 69, 837842.

Barbério, A., Barros, L., Voltolini, J., Mello, M., 2009. Evaluation of the cytotoxic and genotoxic potential of water from the River Paraíba do Sul, in Brazil, with the Allium cepa L. test. Brazilian Journal of Biology 69, 837842.

Barbério, A., Voltolini, J., Mello, M., 2011. Standardization of bulb and root sample sizes for the Allium cepa test. Ecotoxicology 20, 927-935.
Barbosa, J., Cabral, T., Ferreira, D., Agnez-Lima, L., de Medeiros, S.B., 2010. Genotoxicity assessment in aquatic environment impacted by the presence of heavy metals. Ecotoxicology and Environmental Safety 73, 320-325.

Barbosa, R., Lapa, N., Lopes, H., Günther, A., Dias, D., Mendes, B., 2014. Biomass fly ashes as low-cost chemical agents for $\mathrm{Pb}$ removal from synthetic and industrial wastewaters. Journal of Colloid and Interface Science 424, 27-36.

Barrera-Díaz, C., Frontana-Uribe, B., Bilyeu, B., 2014. Removal of organic pollutants in industrial wastewater with an integrated system of copper electrocoagulation and electrogenerated $\mathrm{H}_{2} \mathrm{O}_{2}$. Chemosphere 105, 160-164.

Baruah, B., Das, M., 1997. Effect of paper mill effluent on plankton population of wetland. Environment and Ecology 15, 770-777.

Behbahani, A., Eghbali, H., Ardjmand, M., Noufal, M.M.M., Williamson, H.C., Sayar, O., 2016. A novel bio-compatible sorbent based on carbon nanostructure modified by porphyrin for heavy metal separation from industrial wastewaters. Journal of Environmental Chemical Engineering 4, 398-404.

Behboodi, B.S., Samadi, L., 2004. Detection of apoptotic bodies and oligonucleosomal DNA fragments in cadmium-treated root apical cells of Allium cepa Linnaeus. Plant Science 167, 411-416.

Bellani, L., Rinallo, C., Bennici, A., 1991. Cyto-morphological alterations in Allium roots induced by surfactants. Environmental and Experimental Botany 31, 179183181185.

Bernardes, P.M., Andrade-Vieira, L.F., Aragão, F.B., Ferreira, A., da Silva Ferreira, M.F., 2015. Toxicity of Difenoconazole and Tebuconazole in Allium cepa. Water, Air, \& Soil Pollution 226, 1-11.

Bertani, R., Biasin, A., Canu, P., Della Zassa, M., Refosco, D., Simionato, F., Zerlottin, M., 2016. Self-heating of dried industrial tannery wastewater sludge induced by pyrophoric iron sulfides formation. Journal of Hazardous Materials 305, 105-114.

Bhat, S.A., Singh, J., Vig, A.P., 2014. Genotoxic assessment and optimization of pressmud with the help of exotic earthworm Eisenia fetida. Environmental Science and Pollution Research 21, 8112-8123.

Bhat, S.A., Singh, J., Vig, A.P., 2015. Vermistabilization of sugar beet (Beta vulgaris L) waste produced from sugar factory using earthworm Eisenia fetida: Genotoxic assessment by Allium cepa test. Environmental Science and Pollution Research 22, 11236-11254.

Bhatta, P., Sakya, S., 2009. Study of mitotic activity and chromosomal behaviour in root meristem of Allium cepa L. treated with magnesium sulphate. Ecoprint: An International Journal of Ecology 15, 83-88.

Bhatti, H.N., Zaman, Q., Kausar, A., Noreen, S., Iqbal, M., 2016. Efficient remediation of $\mathrm{Zr}(\mathrm{IV})$ using citrus peel waste biomass: Kinetic, equilibrium and thermodynamic studies. Ecological Engineering 95, 216-228. 
Bianchi, J., Casimiro Fernandes, T.C., Marin-Morales, M.A., 2016. Induction of mitotic and chromosomal abnormalities on Allium cepa cells by pesticides imidacloprid and sulfentrazone and the mixture of them. Chemosphere 144, 475-483.

Bianchi, J., Espindola, E.L.G., Marin-Morales, M.A., 2011. Genotoxicity and mutagenicity of water samples from the Monjolinho River (Brazil) after receiving untreated effluents. Ecotoxicology and Environmental Safety 74, 826-833.

Bianchi, J., Mantovani, M.S., Marin-Morales, M.A., 2015. Analysis of the genotoxic potential of low concentrations of Malathion on the Allium cepa cells and rat hepatoma tissue culture. Journal of Environmental Sciences 36, 102-111.

Bidau, C.J., Amat, A.G., Yajía, M., Martí, D.A., Riglos, A.G., Silvestroni, A., 2004. Evaluation of the genotoxicity of aqueous extracts of Ilex paraguariensis St. Hil.(Aquifoliaceae) using the Allium test. Cytologia 69, 109-117.

Bilal, M., Asgher, M., Iqbal, M., Hu, H., Zhang, X., 2016 . Chitosan beads immobilized manganese peroxidase catalytic potential for detoxification and decolorization of textile effluent. International Journal of Biological Macromolecules 89, 181-189.

Bilal, M., Asgher, M., Shahid, M., 2016b. Enhanced Catalytic Potentiality of Ganoderma lucidum IBL-05 Manganese Peroxidase Immobilized on Sol-Gel Matrix. Journal of Molecular Catalysis B: Enzymatic 128, 82-93.

Bilal, M., Asgher, M., Shahid, M., Bhatti, H.N., $2016 \mathrm{c}$. Characteristic features and dye degrading capability of agar-agar gel immobilized manganese peroxidase. International Journal of Biological Macromolecules 86, 728-740.

Bilal, M., Iqbal, M., Hu, H., Zhang, X., 2016d. Mutagenicity and cytotoxicity assessment of biodegraded textile effluent by Ca-alginate encapsulated manganese peroxidase. Biochemical Engineering Journal 109, 153-161.

Bilal, M., Iqbal, M., Hu, H., Zhang, X., 2016e. Mutagenicity and cytotoxicity assessment of biodegraded textile effluent by Ca-alginate encapsulated manganese peroxidase. Biochemical Engineering Journal 109, 153-161.

Bilal, M., Iqbal, M., Hu, H., Zhang, X., 2016f. Mutagenicity, cytotoxicity and phytotoxicity evaluation of biodegraded textile effluent by fungal ligninolytic enzymes. Water Science and Technology 73(10), 2332-2344.

Bittencourt De Souza, L.F., Laughinghouse IV, H.D., Pastori, T., Tedesco, M., Kuhn, A.W., Canto-Dorow, T.S.D., Tedesco, S.B., 2010. Genotoxic potential of aqueous extracts of Artemisia verlotorum on the cell cycle of Allium cepa. International Journal of Environmental Studies 67, 871877.

Blagojević, J., Stamenković, G., Vujošević, M., 2009. Potential genotoxic effects of melted snow from an urban area revealed by the Allium cepa test. Chemosphere 76, 13441347.
Blanc, L.R., Moreira, F.d.S., Gonçalves, A.M., Manchester, R.S.S.A., Baroni, L., Faria, M.C.d.S., Bomfeti, C.A., Barbosa, F., Rodrigues, J.L., 2013. Contamination in a Brazilian River: A Risk of Exposure to Untreated Effluents. Journal of Environmental Quality 42, 1596-1601.

Bohórquez-Echeverry, P., Duarte-Castañeda, M., León-López, N., Caicedo-Carrascal, F., Vásquez-Vásquez, M., CamposPinilla, C., 2012. Selection of a bioassay battery to assess toxicity in the affluents and effluents of three watertreatment plants. Universitas Scientiarum 17, 152-166.

Bokhari, T.H., Abbas, W., Munir, M., Zuber, M., Usman, M., Iqbal, M., Bhatti, I.A., Bukhari, I.H., Khan, M.K., 2015. Impact of $\mathrm{UV} / \mathrm{TiO}_{2} / \mathrm{H}_{2} \mathrm{O}_{2}$ on Degradation of Disperse Red F3BS. Asian Journal of Chemistry 27, 2825.

Bolle, P., Mastrangelo, S., Tucci, P., Evandri, M.G., 2004. Clastogenicity of atrazine assessed with the Allium cepa test. Environmental and Molecular Mutagenesis 43, 137141.

Boorman, G.A., 1999. Drinking water disinfection byproducts: review and approach to toxicity evaluation. Environmental Health Perspectives 107, 207.

Bordes, M.C., Vicent, M., Moreno, R., García-Montaño, J., Serra, A., Sánchez, E., 2015. Application of plasma-sprayed TiO2 coatings for industrial (tannery) wastewater treatment. Ceramics International 41, 14468-14474.

Bouatay, F., Meksi, N., Adeel, S., Salah, F., Mhenni, F., 2016. Dyeing Behavior of the Cellulosic and Jute Fibers with Cationic Dyes: Process Development and Optimization Using Statistical Analysis. Journal of Natural Fibers 13, 423-436.

Bratu, M.M., Doroftei, E., Negreanu-Pirjol, T., Hostina, C., Porta, S., 2012. Determination of antioxidant activity and toxicity of Sambucus nigra fruit extract using alternative methods. Food Technology and Biotechnology 50, 177 182.

Brkanac, S.R., Vujčić, V., Cvjetko, P., Baković, V., Oreščanin, V., 2014. Removal of landfill leachate toxicity and genotoxicity by two treatment methods. Archives of Industrial Hygiene and Toxicology 65, 89-99.

Cabaravdic, M., 2010. Induction of chromosome aberrations in the Allium cepa test system caused by the exposure of cells to benzo (a) pyrene. Medical Archives 64, 215.

Camacho-Muñoz, D., Martín, J., Santos, J.L., Aparicio, I., Alonso, E., 2014. Concentration evolution of pharmaceutically active compounds in raw urban and industrial wastewater. Chemosphere 111, 70-79.

Camparoto, M.L., de Oliveira Teixeira, R., Mantovani, M.S., Vicentini, V.E.P., 2002. Effects of Maytenus ilicifolia Mart. and Bauhinia candicans Benth infusions on onion roottip and rat bone-marrow cells. Genetics and Molecular Biology 25, 85-90.

Carbajo, J.B., Perdigón-Melón, J.A., Petre, A.L., Rosal, R., Letón, P., García-Calvo, E., 2015. Personal care product preservatives: Risk assessment and mixture toxicities with an industrial wastewater. Water Research 72, 174185. 
Carita, R., Marin-Morales, M., 2008. Induction of chromosome aberrations in the Allium cepa test system caused by the exposure of seeds to industrial effluents contaminated with azo dyes. Chemosphere 72, 722-725.

Carruyo, I., Fernández, Y., Marcano, L., Montiel, X., Torrealba, Z., 2008. Correlation of toxicity with lead content in root tip cells (Allium cepa L.). Biological Trace Element Research 125, 276-285.

Çavuşoğlu, D., Tabur, S., Çavuşoğlu, K., 2016. The Effects of Aloe vera L. Leaf Extract on Some Physiological and Cytogenetical Parameters in Allium cepa L. Seeds Germinated under Salt Stress. Cytologia 81, 103-110.

Çavuşoğlu, K., Kaya, A., Yilmaz, F., Yalçin, E., 2012a. Effects of cypermethrin on Allium cepa. Environmental toxicology 27, 583-589.

Çavuşoğlu, K., Yalçın, E., Türkmen, Z., Yapar, K., Çavuşoğlu, K., Çiçek, F., 2011. Investigation of Toxic Effects of the Glyphosate on Allium cepa. Tarım Bilimleri DergisiJournal o f Agricultural Sciences 17, 131-142.

Çavuşoğlu, K., Yalçin, E., Türkmen, Z., Yapar, K., Sağir, S., 2012b. Physiological, anatomical, biochemical, and cytogenetic effects of thiamethoxam treatment on Allium cepa (amaryllidaceae) L. Environmental Toxicology 27, 635-643.

Çelik, T.A., Aslantürk, O., 2009. Investigation of cytotoxic and genotoxic effects of Ecballium elaterium juice based on Allium test. Methods and Findings in Experimental and Clinical Pharmacology 31, 591-596.

Chae, J.-S., Choi, S.-A., Kim, Y.-H., Oh, S.-C., Ryu, C.-K., Ohm, T.I., 2016. Experimental study of fry-drying and melting system for industrial wastewater sludge. Journal of Hazardous Materials 313, 78-84.

Chahal, V., Nagpal, A., Katnoria, J.K., 2012. Genotoxicity Evaluation of Soil Sample from Agricultural Field under Wheat Cultivation. Botany Research Internationa 5, 1-3.

Chaik, M., El Fadeli, S., Sedki, A., Lekouch, N., 2011. Mutagenic and genotoxic effects of wastewater detected by the Allium test. Mediterranean Journal of Chemistry 1, 163172.

Chakraborty, R., Mukherjee, A., 2010. Technical note: Vetiver can grow on coal fly ash without DNA damage. International Journal of Phytoremediation 13, 206-214.

Chakraborty, R., Mukherjee, A.K., Mukherjee, A., 2009. Evaluation of genotoxicity of coal fly ash in Allium cepa root cells by combining comet assay with the Allium test. Environmental monitoring and assessment 153, 351357.

Chandra, S., Chauhan, L., Murthy, R., Saxena, P., Pande, P., Gupta, S., 2005. Comparative biomonitoring of leachates from hazardous solid waste of two industries using Allium test. Science of the total environment 347, 46-52.

Chandra, S., Chauhan, L., Pande, P., Gupta, S., 2004. Cytogenetic effects of leachates from tannery solid waste on the somatic cells of Vicia faba. Environmental Toxicology 19, 129-133.

Chandra, S., Gupta, S., 2002. Genotoxicity of leachates of tannery solid waste in root meristem cells of Allium cepa.
Journal of Ecophysiology and Occupational Health 2, 225234.

Chang, L., Meier, J., Smith, M., 1997. Application of plant and earthworm bioassays to evaluate remediation of a leadcontaminated soil. Archives of Environmental Contamination and Toxicology 32, 166-171.

Chaparro, T., Botta, C., Pires, E., 2010. Biodegradability and toxicity assessment of bleach plant effluents treated anaerobically. Water Science and Technology 62, 13121319.

Chaparro, T., Pires, E., 2011. Anaerobic treatment of cellulose bleach plant wastewater: chlorinated organics and genotoxicity removal. Brazilian Journal of Chemical Engineering 28, 625-638.

Chaparro, T., Pires, E., 2015. Post-treatment of anaerobic effluent by ozone and ozone/UV of a kraft cellulose pulp mill. Water Science and Technology 71, 382-389.

Chauhan, L., Gupta, S., 2005a. Combined cytogenetic and ultrastructural effects of substituted urea herbicides and synthetic pyrethroid insecticide on the root meristem cells of Allium cepa. Pesticide Biochemistry and Physiology 82, 27-35.

Chauhan, L., Saxena, P., Gupta, S., 1999. Cytogenetic effects of cypermethrin and fenvalerate on the root meristem cells of Allium cepa. Environmental and experimental botany 42, 181-189.

Chauhan, L., Saxena, P., Sundararaman, V., Gupta, S., 1998. Diuron-induced cytological and ultrastructural alterations in the root meristem cells of Allium cepa. Pesticide Biochemistry and Physiology 62, 152-163.

Chauhan, L.K.S., Dikshith, T.S.S., Sundararaman, V., 1986. Effect of deltamethrin on plant cells I. Cytological effects on the root meristems of Allium cepa. Mutation Research/Genetic Toxicology 171, 25-30.

Chauhan, L.K.S., Gupta, S.K., 2005b. Combined cytogenetic and ultrastructural effects of substituted urea herbicides and synthetic pyrethroid insecticide on the root meristem cells of Allium cepa. Pesticide Biochemistry and Physiology 82, 27-35.

Cheballah, K., Sahmoune, A., Messaoudi, K., Drouiche, N., Lounici, H., 2015. Simultaneous removal of hexavalent chromium and COD from industrial wastewater by bipolar electrocoagulation. Chemical Engineering and Processing: Process Intensification 96, 94-99.

Chong, M.N., Tneu, Z.Y., Poh, P.E., Jin, B., Aryal, R., 2015. Synthesis, characterisation and application of TiO2zeolite nanocomposites for the advanced treatment of industrial dye wastewater. Journal of the Taiwan Institute of Chemical Engineers 50, 288-296.

Chou, P.-H., Liu, T.-C., Lin, Y.-L., 2014. Monitoring of xenobiotic ligands for human estrogen receptor and aryl hydrocarbon receptor in industrial wastewater effluents. Journal of Hazardous Materials 277, 13-19.

Christofoletti, C.A., Francisco, A., Fontanetti, C.S., 2012. Biosolid soil application: toxicity tests under laboratory conditions. Applied and Environmental Soil Science 2012. http://dx.doi.org/10.1155/2012/518206. 
Christofoletti, C.A., Pedro-Escher, J., Fontanetti, C.S., 2013. Assessment of the genotoxicity of two agricultural residues after processing by diplopods using the Allium cepa assay. Water, Air, \& Soil Pollution 224, 1-14.

Chukwujekwu, J.C., Van Staden, J., 2014. Cytotoxic and genotoxic effects of water extract of Distephanus angulifolius on Allium cepa Linn. South African Journal of Botany 92, 147-150.

Corrêa Martins, M.N., Souza, V.V.d., Silva Souza, T.d., 2016. Cytotoxic, genotoxic and mutagenic effects of sewage sludge on Allium cepa. Chemosphere 148, 481-486.

Cotelle, S., Masfaraud, J.F., Férard, J.F., 1999. Assessment of the genotoxicity of contaminated soil with the Allium/Vicia-micronucleus and the Tradescantiamicronucleus assays. Mutation Research/Fundamental and Molecular Mechanisms of Mutagenesis 426, 167-171.

Crebelli, R., Conti, L., Marchini, S., Monarca, S., Feretti, D., Zerbini, I., Zani, C., Veschetti, E., Cutilli, D., Ottaviani, M., 2002. [Genotoxic and ecotoxic effects of urban waste water disinfected with sodium hypochlorite or peracetic acid]. Annali di igiene: medicina preventiva e di comunita 15, 277-302.

Crebelli, R., Conti, L., Monarca, S., Feretti, D., Zerbini, I., Zani, C., Veschetti, E., Cutilli, D., Ottaviani, M., 2005. Genotoxicity of the disinfection by-products resulting from peracetic acid-or hypochlorite-disinfected sewage wastewater. Water Research 39, 1105-1113.

da Costa Filho, B.M., da Silva, V.M., Silva, J.d.O., Hora Machado, A.E.d., Trovó, A.G., 2016. Coupling coagulation, flocculation and decantation with photo-Fenton process for treatment of industrial wastewater containing fipronil: Biodegradability and toxicity assessment. Journal of Environmental Management 174, 71-78.

da Costa Machado Matos Carvalho, I.M., Cavalcante, A.A.M., Dantas, A.F., Pereira, D.L.A., Rocha, F.C.C., Oliveira, F.M., Da Silva, J., 2011. Environmental mutagenicity and toxicity caused by sodium metabisulfite in sea shrimp harvesting in PiauÃ, Brazil. Chemosphere 82, 1056-1061.

da Costa, T.C., de Brito, K.C.T., Rocha, J.A.V., Leal, K.A., Rodrigues, M.L.K., Minella, J.P.G., Matsumoto, S.T., Vargas, V.M.F., 2011. Runoff of genotoxic compounds in river basin sediment under the influence of contaminated soils. Ecotoxicology and Environmental Safety 75(1), 6372.

da Lima, R., Feitosa, L.O., Grillo, R., Pereira, A.E., Fraceto, L.F., 2012. Evaluation of the genotoxicity of polymeric microparticles containing ametryn herbicide. Journal of Environmental Science and Engineering. A 1, 10.

da Silva Francisconi, M., De Bona, A., da Silveira, F.Z., Geremias, R., Pich, C.T., 2013. Use of coal mining waste for river water treatment and evaluation of this process using physicochemical parameters and bioassays. Ecotoxicology and Environmental Contamination 8, 4551.

da Silva, R.M.G., do Amaral, E.A., de Oliveira Moraes, V.M., Silva, L.P., 2013. Determination of heavy metals and genotoxicity of water from an artesian well in the city of
Vazante-MG, Brazil. African Journal of Biotechnology 12, 6938.

Dash, S., Panda, K.K., Panda, B.B., 1988. Biomonitoring of low levels of mercurial derivatives in water and soil by Allium micronucleus assay. Mutation Research/Environmental Mutagenesis and Related Subjects 203, 11-21.

Dayana, G.P., Joela, O.L., Tatiana, R.C., 2012. Removal of organic matter and toxicity in hospital wastewaters by ozone. Dyna 79, 105-115.

de Andrade, L.R., Sandin Brito, A., de Souza Melero, A.M.G., Zanin, H., José Ceragioli, H., Baranauskas, V., Silva Cunha, K., Pierre Irazusta, S., 2014. Absence of mutagenic and recombinagenic activity of multi-walled carbon nanotubes in the Drosophila wing-spot test and Allium cepa test. Ecotoxicology and Environmental Safety 99, 92-97.

de Campos Ventura-Camargo, B., Marin-Morales, M.A., 2016. Micronuclei and chromosome aberrations derived from the action of Atrazine herbicide in Allium cepa meristematic cells. SDRP Journal of Earth Sciences \& Environmental Studies 1, 1-7.

De Lima, R., Feitosa, L., Pereira, A.d.E.S., De Moura, M.R., Aouada, F.A., Mattoso, L.H.C., Fraceto, L.F., 2010. Evaluation of the genotoxicity of chitosan nanoparticles for use in food packaging films. Journal of Food Science 75, N89-N96.

de Maio Lacerda, P.M., Asano, A., Morita, A., Balsamo, P., Nascimento, R., Teixeira, E., Irazusta, S., 2014. Ecotoxicological assessment of irrigation water for vegetables in a watershed region of Greater São Paulo. Ecotoxicology and Environmental Contamination 9, 2127.

De Marco, A., Romanelli, M., Stazi, M.A., Vitagliano, E., 1986. Induction of micronucleated cells in Vicia faba and Allium cepa root tips treated with nitrilotriacetic acid (NTA). Mutation Research/Genetic Toxicology 171, 145-148.

de Oliveira Meneguetti, D.U., da Silva, F.C., Zan, R.A., Ramos, L.J., 2012. Adaptation of the micronucleus technique in Allium cepa, for mutagenicity analysis of the Jamari river valley, western Amazon, Brazil. JJournal of Environmental and Analytical Toxicology 2, 21610525.1000127.

de Souza Pohren, R., da Costa, T.C., Vargas, V.M.F., 2013. Investigation of sensitivity of the Allium cepa test as an alert system to evaluate the genotoxic potential of soil contaminated by heavy metals. Water, Air, \& Soil Pollution 224, 1-10.

Defaveri, T.M., da Silveira, F.Z., Bortolotto, T., Geremias, R., Zocche, J.J., Pich, C.T., 2009. Evaluation of acid mine drainage treatment using Artemia sp. and Allium cepa as bioindicators of toxicity and genotoxicity, 26 th Annual American Society of Mining and Reclamation Meeting, Proceedings America Society of Mining and Reclamation, pp. 110-110.

do Canto, T., Pich, C.T., Geremias, R., 2013. Bioensaio de toxicidade em percolados no aterro sanitário do 
município de Araranguá (Santa Catarina, Brasil). Revista Biociências 19, 53.

Düsman, E., Luzza, M., Savegnago, L., Lauxen, D., Vicentini, V.E.P., Tonial, I.B., Sauer, T.P., 2014. Allium cepa L. as a bioindicator to measure cytotoxicity of surface water of the Quatorze River, located in Francisco Beltrão, Paraná, Brazil. Environmental Monitoring and Assessment 186, 1793-1800.

Dutta, S., 1999. Study of the physico-chemical properties of effluent of the paper mill that affected the paddy plants. Journal of Environment and Pollution 6, 181-188.

Edemaa, N.E., Okungbowa, F.I., 2011. Fungi isolated from produced water and water-soluble fraction of crude oil, Series B: Biological Sciences, p. 163.

Egito, L.C.M., Medeiros, M.G., Medeiros, S.R.B., Agnez-Lima, L.F., 2007. Cytotoxic and genotoxic potential of surface water from the Pitimbu river, northeastern/RN Brazil. Genetics and Molecular Biology 30, 435-441.

El-Ghamery, A., El-Kholy, M., Abou El-Yousser, M., 2003. Evaluation of cytological effects of $\mathrm{Zn2}+$ in relation to germination and root growth of Nigella sativa L. and Triticum aestivum L. Mutation Research/Genetic Toxicology and Environmental Mutagenesis 537, 29-41.

El-Shahaby, O., Migid, H.M.A., Soliman, M., Mashaly, I., 2003. Genotoxicity screening of industrial wastewater using the allium chromosome aberration assay. Pakistan Journal of Biological Sciences 6, 23-28.

Eremektar, G., Selcuk, H., Meric, S., 2007. Investigation of the relation between COD fractions and the toxicity in a textile finishing industry wastewater: Effect of preozonation. Desalination 211, 314-320.

Eren, Y., Özata, A., 2014. Determination of mutagenic and cytotoxic effects of Limonium globuliferum aqueous extracts by Allium, Ames, and MTT tests. Revista Brasileira de Farmacognosia 24, 51-59.

Espinoza-Quiñones, F., Szymanski, N., Palácio, S., Módenes, A., Rizzutto, M.d.A., Silva Jr, F., Oliveira, A., Oro, A., Martin, N., 2009. Inhibition effect on the Allium cepa L. root growth when using hexavalent chromium-doped river waters. Bulletin of Environmental Contamination and Toxicology 82, 767-771.

Evandri, M.G., Mastrangelo, S., Costa, L.G., Bolle, P., 2003. In vitro assessment of mutagenicity and clastogenicity of BDE-99, a pentabrominated diphenyl ether flame retardant. Environmental and Molecular Mutagenesis 42, 85-90.

Evandri, M.G., Tucci, P., Bolle, P., 2000. Toxicological evaluation of commercial mineral water bottled in polyethylene terephthalate: a cytogenetic approach with Allium cepa. Food Additives \& Contaminants 17, 1037 1045.

Evseeva, T., Măstrenko, T., Geras' kin, S., Belykh, E., 2005. The influence of cadmium and potassium on the level of cytogenetic effects induced by thorium-232 in Allium cepa root meristem. Tsitologiia i Genetika 40, 50-58.

Evseeva, T.I., Gerasâkin, S.A., Shuktomova, I.I., 2003. Genotoxicity and toxicity assay of water sampled from a radium production industry storage cell territory by means of Allium-test. Journal of environmental radioactivity $68,235-248$.

Expósito, A.J., Durán, A., Monteagudo, J.M., Acevedo, A., 2016. Solar photo-degradation of a pharmaceutical wastewater effluent in a semi-industrial autonomous plant. Chemosphere 150, 254-257.

Fajardo, D.R.M., Magdugo, R.P., Deiparine, I.G., 2015. Cytological Effects of Sediment Elutriate from Tambis River System, Barobo, Surigao Del Sur in Allium cepa Root Meristem. Journal of Cytology \& Histology 2015, 8.

Fatima, R.A., Ahmad, M., 2005. Certain antioxidant enzymes of Allium cepa as biomarkers for the detection of toxic heavy metals in wastewater. Science of The Total Environment 346, 256-273.

Fatima, R.A., Ahmad, M., 2006a. Genotoxicity of industrial wastewaters obtained from two different pollution sources in northern India: a comparison of three bioassays. Mutation Research/Genetic Toxicology and Environmental Mutagenesis 609, 81-91.

Fatima, R.A., Ahmad, M., 2006b. Allium cepa derived EROD as a potential biomarker for the presence of certain pesticides in water. Chemosphere 62, 527-537.

Fawole, O., Yekeen, T., Ayandele, A., Akinboro, A., Azeez, M., Adewoye, S., 2008. Polluted Alamuyo River: Impacts on surrounding wells, microbial attributes and toxic effects on Allium cepa root cells. African Journal of Biotechnology 7, 12.

Fazili, N.A., Ahmad, M., 2014. In vitro analysis of the phytotoxic and genotoxic potential of Aligarh wastewater and Mathura refinery wastewater. Toxicology Reports 1, 981-986.

Fenech, M., 2002. Biomarkers of genetic damage for cancer epidemiology. Toxicology 181, 411-416.

Feretti, D., Zerbini, I., Ceretti, E., Villarini, M., Zani, C., Moretti, M., Fatigoni, C., Orizio, G., Donato, F., Monarca, S., 2008. Evaluation of chlorite and chlorate genotoxicity using plant bioassays and in vitro DNA damage tests. Water Research 42, 4075-4082.

Feretti, D., Zerbini, I., Zani, C., Ceretti, E., Moretti, M., Monarca, S., 2007. Allium cepa chromosome aberration and micronucleus tests applied to study genotoxicity of extracts from pesticide-treated vegetables and grapes. Food Additives and Contaminants 24, 561-572.

Fernandes, R., Matos, C., Alvarenga, P., Palma, P., Soares, A., Palma, V., Barbosa, I., 2010. Evaluation of surface water quality using an ecotoxicological approach: a case study of the Alqueva Reservoir (Portugal). Environmental Science and Pollution Research 17(3), 703-16

Fernandes, T.C.C., Mazzeo, D.E.C., Marin-Morales, M.A., 2007 a. Mechanism of micronuclei formation in polyploidizated cells of Allium cepa exposed to trifluralin herbicide. Pesticide Biochemistry and Physiology 88, 252-259.

Fernandes, T.C.C., Mazzeo, D.E.C., Marin-Morales, M.A., 2007b. Mechanism of micronuclei formation in polyploidizated cells of Allium cepa exposed to trifluralin herbicide. Pesticide Biochemistry and Physiology 88, 252-259. 
Fernández Freire, P., Peropadre, A., Rosal, R., Pérez Martín, J.M., Hazen, M.J., 2015. Toxicological assessment of third generation (G3) poly (amidoamine) dendrimers using the Allium cepa test. Science of The Total Environment doi:10.1016/j.scitotenv.2015.07.137.

Firbas, P., Amon, T., 2013. Allium chromosome aberration test for evaluation effect of cleaning municipal water with Constructed Wetland (CW) in Sveti Tomaž, Slovenia. Journal of Bioremediation and Biodegradation 4, 189193.

Firbas, P., Amon, T., 2014. Chromosome damage studies in the onion plant Allium cepa L. Caryologia 67, 25-35.

Fiskejo, G., 1985. The Allium test as a standard in environmental monitoring. Hereditas 102, 99-112.

Fiskesjo, G., 1981. Allium test on copper in drinking water. Vatten 37, 233-240.

Fiskesjo, G., 1988. The Allium test--an alternative in environmental studies: the relative toxicity of metal ions. Mutation Research/Fundamental and Molecular Mechanisms of Mutagenesis 197, 243-260.

Fiskesjo, G., 1993. The Allium test in wastewater monitoring. Environmental toxicology and water quality 8, 291-298.

Fisun, K., Rasgele, P.G., 2009. Genotoxic effects of raxil on root tips and anthers of Allium cepa L. Caryologia 62, 1-9.

Flores Navarro, F., Scicia Oliveira, B., Dani Campos Pereira, F., de Campos Severi Aguiar, G.D., Marin Morales, M.A., 2015. The toxic effects of Cacti-Nea ${ }^{\mathrm{TM}}$ on germination of $A$. сера. Toxicology Letters 238, S70-S71.

Frank, V., Harangozó, M., 1994. Heavy metals in industrial wastewater determined by radionuclide X-ray fluorescence analysis and their effects on Allium cepa root tip cells. Journal of Radioanalytical and Nuclear Chemistry 187, 137-141.

Freire, P.F., Martín, J.M.P., Peropadre, A., Aquilino, M., Rosal, R., Hazen, M.J., 2014. Toxic effects induced by poly(amidoamine) dendrimers in Allium cepa. Toxicology Letters 229, Supplement, S190.

Frescura, V.D.-S., Laughinghouse IV, H.D., Tedesco, S.B., 2012. Antiproliferative effect of the tree and medicinal species Luehea divaricata on the Allium cepa cell cycle. Caryologia 65, 27-33.

Gajalakshmi, K., Ruban, P., 2014. Evaluation of physico chemical parameters and cytotoxic effect of Orathupalayam dam in Tirupur District. International Journal of Agricultural Policy and Research 2, 191-197.

Gana, J.M., Ordóñez, R., Zampini, C., Hidalgo, M., Meoni, S., Isla, M.I., 2008. Industrial effluents and surface waters genotoxicity and mutagenicity evaluation of a river of Tucuman, Argentina. Journal of Hazardous Materials 155, 403-406.

Gangadhara, R., Prasad, N., 2016. Studies on optimization of transesterification of certain oils to produce biodiesel. Chemistry International 2, 59-69.

Garaj-Vrhovac, V., Oreščanin, V., Gajski, G., Gerić, M., Ruk, D., Kollar, R., Brkanac, S.R., Cvjetko, P., 2013. Toxicological characterization of the landfill leachate prior/after chemical and electrochemical treatment: a study on human and plant cells. Chemosphere 93, 939-945.

Gatsios, E., Hahladakis, J.N., Gidarakos, E., 2015. Optimization of electrocoagulation (EC) process for the purification of a real industrial wastewater from toxic metals. Journal of Environmental Management 154, 117-127.

Gatta, G., Libutti, A., Gagliardi, A., Beneduce, L., Brusetti, L., Borruso, L., Disciglio, G., Tarantino, E., 2015. Treated agro-industrial wastewater irrigation of tomato crop: Effects on qualitative/quantitative characteristics of production and microbiological properties of the soil. Agricultural Water Management 149, 33-43.

Geras'kin, S., Oudalova, A., Michalik, B., Dikareva, N., Dikarev, V., 2011. Geno-toxicity assay of sediment and water samples from the Upper Silesia post-mining areas, Poland by means of Allium-test. Chemosphere 83(8), 1133-46.

Geras'kin, S., Oudalova, A., Michalik, B., Dikareva, N., Dikarev, V., 2011. Geno-toxicity assay of sediment and water samples from the Upper Silesia post-mining areas, Poland by means of Allium-test. Chemosphere 83, 11331146.

Geremias, R., Bortolotto, T., Wilhelm-Filho, D., Pedrosa, R.C., de Fávere, V.T., 2012. Efficacy assessment of acid mine drainage treatment with coal mining waste using Allium cepa L. as a bioindicator. Ecotoxicology and Environmental Safety 79, 116-121.

Geremias, R., De Fávere, V.T., Pedrosa, R.C., Fattorini, D., 2011. Bioaccumulation and adverse effects of trace metals and polycyclic aromatic hydrocarbons in the common onion Allium cepa as a model in ecotoxicological bioassays. Chemistry and Ecology 27, 515-522.

Geremias, R., Fattorini, D., Fávere, V.T., Pedrosa, R.C., 2010. Bioaccumulation and toxic effects of copper in common onion Allium cepa L. Chemistry and Ecology 26, 19-26.

Ghodake, G., Seo, Y.D., Lee, D.S., 2011. Hazardous phytotoxic nature of cobalt and zinc oxide nanoparticles assessed using Allium cepa. Journal of hazardous materials 186, 952-955.

Ghosh, M., Bhadra, S., Adegoke, A., Bandyopadhyay, M., Mukherjee, A., 2015. MWCNT uptake in Allium cepa root cells induces cytotoxic and genotoxic responses and results in DNA hyper-methylation. Mutation Research/Fundamental and Molecular Mechanisms of Mutagenesis 774, 49-58.

Glasencnik, E., Ribaric-Lasnik, C., Muller, M., Grill, D., Batic, F., 2002. Impact of air pollution on genetic material of the shallot (Allium cepa L. var. ascalonicum), exposed in the vicinity of major slovene local emission sources in 1999. Phyton-Horn 42, 237-250.

Glasenčnik, E., Ribarič-Lasnik, C., Savinek, K., Zaluberšek, M., Mueller, M., Batič, F., 2004. Impact of air pollution on genetic material of shallot (Allium cepa L. var. ascalonicum) exposed at differently polluted sites in Slovenia. Journal of Atmospheric Chemistry 49, 363-376.

Glińska, S., Bartczak, M., Oleksiak, S., Wolska, A., Gabara, B., Posmyk, M., Janas, K., 2007. Effects of anthocyanin-rich 
extract from red cabbage leaves on meristematic cells of Allium cepa L. roots treated with heavy metals. Ecotoxicology and Environmental Safety 68, 343-350.

Glińska, S., Gabara, B., 2011. The effects of the anthocyaninrich extract from red cabbage leaves on Allium cepa L. root tip cell ultrastructure. Ecotoxicology and Environmental Safety 74, 93-98.

Goh, S., Zhang, J., Liu, Y., Fane, A.G., 2015. Membrane Distillation Bioreactor (MDBR) - A lower Green-HouseGas (GHG) option for industrial wastewater reclamation. Chemosphere 140, 129-142.

Gomez-Mendikute, A., Cajaraville, M., 2003. Comparative effects of cadmium, copper, paraquat and benzo [a] pyrene on the actin cytoskeleton and production of reactive oxygen species (ROS) in mussel haemocytes. Toxicology in Vitro 17, 539-546.

Gömürgen, A.N., 2005. Cytological effect of the potassium metabisulphite and potassium nitrate food preservative on root tips of Allium cepa L. Cytologia 70, 119-128.

Gonçalves, P.S., Oliveira, C.S., Mendes, M.P., Duarte, M.N., Rodrigues, W.C., 2015. Efficiency of the aquatic macrophyte salvinia auriculata in purification of urban effluents, validated by allium test (Allium Cepa L.). Revista Eletrônica TECCEN 8, 29-35.

Goncharuk, V., Chebotareva, R., Kovalenko, V., Pasichnaya, E., 2011. The Biotesting of tap water treated with silicon mineral using plant test organisms. Journal of Water Chemistry and Technology 33, 328-332.

González, P.S., Maglione, G.A., Giordana, M., Paisio, C.E., Talano, M.A., Agostini, E., 2012. Evaluation of phenol detoxification by Brassica napus hairy roots, using Allium cepa test. Environmental Science and Pollution Research 19, 482-491.

Goujon, E., Richard, C., Goupil, P., Ledoigt, G., 2015. Cytotoxicity on Allium cepa of the two main sulcotrione photoproducts, $\quad$ xanthene-1,9-dione-3,4-dihydro-6methylsulphonyl and 2-chloro-4-mesylbenzoic acid. Pesticide Biochemistry and Physiology 124, 37-42.

Goujon, E., Sta, C., Trivella, A., Goupil, P., Richard, C., Ledoigt, G., 2014. Genotoxicity of sulcotrione pesticide and photoproducts on Allium cepa root meristem. Pesticide Biochemistry and Physiology 113, 47-54.

Grillo, R., Clemente, Z., Oliveira, J.L.d., Campos, E.V.R., Chalupe, V.C., Jonsson, C.M., Lima, R.d., Sanches, G., Nishisaka, C.S., Rosa, A.H., Oehlke, K., Greiner, R., Fraceto, L.F., 2015. Chitosan nanoparticles loaded the herbicide paraquat: The influence of the aquatic humic substances on the colloidal stability and toxicity. Journal of Hazardous Materials 286, 562-572.

Grippa, G.d.A., Nati, N., Matsumoto, S.T., 2012. Evaluation of water samples from a river by cytologic analysis in Allium cepa. Cytologia 77, 3-9.

Grisales Penagos, D., Ortega López, J., Rodriguez Chaparro, T., 2012. Remoción de la materia orgánica y toxicidad en aguas residuales hospitalarias aplicando ozono. Dyna 79, 109-115.
Grisolia, C.K., de Oliveira, A.B.B., Bonfim, H., de NazarÃ@) Klautau-GuimarÃ£es, M., 2005. Genotoxicity evaluation of domestic sewage in a municipal wastewater treatment plant. Genetics and Molecular Biology 28, 334-338.

Grisolia, C.K., Takahashi, C.S., 1991. Evaluation of mutagenic effect of the antihypertensive drug methyldopa (Aldomet) on mammalian systems in vivo and in vitro and on Allium cepa. Mutation Research/Genetic Toxicology 259, 127-132.

Grover, I., Kaur, S., 1999. Genotoxicity of wastewater samples from sewage and industrial effluent detected by the Allium root anaphase aberration and micronucleus assays. Mutation Research/Fundamental and Molecular Mechanisms of Mutagenesis 426, 183-188.

Gulzar, T., Adeel, S., Hanif, I., Rehman, F., Hanif, R., Zuber, M., Akhtar, N., 2015. Eco-friendly dyeing of gamma ray induced cotton using natural quercetin extracted from acacia bark (A. nilotica). Journal of Natural Fibers 12, 494-504.

Gupta, A., 1997. Pollution load of paper mill effluent and its impact on biological environment. Journal of Ecotoxicology \& Environmental Monitoring 7, 101-112.

Gupta, A.K., Ahmad, M., 2012a. Assessment of cytotoxic and genotoxic potential of refinery waste effluent using plant, animal and bacterial systems. Journal of Hazardous Materials 30, 92-99.

Gupta, A.K., Ahmad, M., 2012b. Effect of refinery waste effluent on tocopherol, carotenoid, phenolics and other antioxidants content in Allium cepa. Toxicology and Industrial Health, 29(7), 652-661.

Gupta, K., Gaumat, S., Mishra, K., 2012. Studies on phytogenotoxic assessment of tannery effluent and chromium on Allium cepa. Journal of Environmental Biology 33, 557.

Guzy, J., Zgórska, A., Ziembińska, A., 2012. Comet assay optimization with Allium cepa as an indicator for ecotoxicological usage. Architecture Civil Engineering Environment 5, 109-116.

Hardy, M., Aufderheide, J., Krueger, H., Mathews, M., Porch, J., Schaefer, E., Stenzel, J., Stedeford, T., 2011. Terrestrial toxicity evaluation of decabromodiphenyl ethane on organisms from three trophic levels. Ecotoxicology and Environmental Safety 74, 703-710.

Haroun, S., Al Shehri, A., 2001. Cytogenetic effects of Calotropis procera extract on Vicia faba L. Cytologia 66, 373-378.

Hassan, G., Yassein, A., 2016. Cytogenotoxicity evaluation of water contami-nated with some textile azo dyes using rapd mark-ers and chromosomal aberrations of onion (Allium cepa) root cells. Egyptian Journal of Genetics And Cytology 43, 7.

Hemachandra, C.K., Pathiratne, A., 2015. Assessing toxicity of copper, cadmium and chromium levels relevant to discharge limits of industrial effluents into inland surface waters using common onion, Allium cepa bioassay. Bulletin of environmental contamination and toxicology 94, 199-203. 
Hemanth Kumar, N.K., Taj, M., Jagannath, S., 2015. Clastogenicity of sugar factory effluent using Allium assay. Research in Plant Biology 5, 10.

Herrero, O., Pérez Martín, J.M., Fernández Freire, P., Carvajal López, L., Peropadre, A., Hazen, M.J., 2012. Toxicological evaluation of three contaminants of emerging concern by use of the Allium cepa test. Mutation Research/Genetic Toxicology and Environmental Mutagenesis 743, 20-24.

Holan, I.O., 2014. Toxic and Genotoxic Effects of Fast Pyrolysis Oils Generated from Three Different Feedstocks Evaluated in an Allium cepa Chromosome Aberration Assay.

Hoshina, M.M., Marin-Morales, M.A., 2009. Micronucleus and chromosome aberrations induced in onion (Allium cepa) by a petroleum refinery effluent and by river water that receives this effluent. Ecotoxicology and Environmental Safety 72, 2090-2095.

Houk, V.S., 1992. The genotoxicity of industrial wastes and effluents:: A review. Mutation Research/Reviews in Genetic Toxicology 277, 91-138.

Howe, J., Wagner, M.R., 1999. Effects of pulpmill effluent irrigation on the distribution of elements in the profile of an arid region soil. Environmental Pollution 105, 129135.

Hu, Q.-Y., Li, M., Wang, C., Ji, M., 2015. Influence of powdered activated carbon addition on water quality, sludge properties, and microbial characteristics in the biological treatment of commingled industrial wastewater. Journal of Hazardous Materials 295, 1-8.

Huang, J., Ou, Y., Yew, T.W.D., Liu, J., Leng, B., Lin, Z., Su, Y., Zhuang, Y., Lin, J., Li, X., Xue, Y., Pan, Y., 2016. Hepatoprotective effects of polysaccharide isolated from Agaricus bisporus industrial wastewater against CCl4induced hepatic injury in mice. International Journal of Biological Macromolecules 82, 678-686.

Husaain, D., Najam-ul-Haq, M., Saeed, A., Jabeen, F., Athar, M., Naeem Ashiq, M., 2015. Synthesis of poly GMA/DVB and its application for the removal of Malachite Green from aqueous medium by adsorption process. Desalination and Water Treatment 53, 2518-2528.

Ilbaş, A.İ., Gönen, U., Yilmaz, S., Dadandi, M.Y., 2012. Cytotoxicity of Aloe vera gel extracts on Allium cepa root tip cells. Turkish Journal of Botany 36, 263-268.

Inceer, H., Beyazoglu, O., Ergul, H.A., 2000. Cytogenetic effects of wastes of copper mine on root tip cells of Allium cepa L. Pakistan Journal of Biological Sciences 3, 10.

Iqbal, J., Cecil, F., Ahmad, K., Iqbal, M., Mushtaq, M., Naeem, M., Bokhari, T., 2013a. Kinetic study of $\mathrm{Cr}$ (III) and $\mathrm{Cr}$ (VI) biosorption using Rosa damascena phy-tomass: a rose waste biomass. Asian Journal of Chemistry 25, 20992103.

Iqbal, J.M., Cecil, F., Ahmad, K., Iqbal, M., Mushtaq, M., Naeem, M.A., Bokhari, T.H., 2013b. Kinetic study of $\mathrm{Cr}$ (III) and Cr(VI) biosorption using Rosa damascena phytomass: a rose waste biomass. Asian Journal of Chemistry 25, 2099.

Iqbal, M., 2016. Vicia faba bioassay for environmental toxicity monitoring: a review. Chemosphere 144, 785-802.
Iqbal, M., Abbas, M., Arshad, M., Hussain, T., Khan, A.U., Masood, N., Tahir, M.A., Hussain, S.M., Bokhari, T.H., Khera, R.A., 2015a. Gamma I Radiation Treatment for Reducing Cytotoxicity and Mutagenicity in Industrial Wastewater. Polish Journal of Environmental Studies 24, 2745-2750.

Iqbal, M., Abbas, M., Arshad, M., Hussain, T., Khan, A.U., Masood, N., Tahir, M.A., Hussain, S.M., Bokhari, T.H., Khera, R.A., 2015b. Gamma radiation treatment for reducing cytotoxicity and mutagenicity in industrial wastewater. Polish Journal of Environmental Studies 24, 2745-2750.

Iqbal, M., Bhatti, I.A., 2014. Re-utilization option of industrial wastewater treated by advanced oxidation process. Pakistan Journal of Agriculture Sciences 51, 1141-1147.

Iqbal, M., Bhatti, I.A., 2015a. Gamma radiation $/ \mathrm{H}_{2} \mathrm{O}_{2}$ treatment of a nonylphenol ethoxylates: Degradation, cytotoxicity, and mutagenicity evaluation. Journal of Hazardous Materials 299, 351-360.

Iqbal, M., Bhatti, I.A., Shahid, M., Jan, N., $2016 a$. Physicochemical characterization, microbial decontamination and shelf life analysis of walnut (Juglans regia L) oil extracted from gamma radiation treated seeds. Biocatalysis and Agricultural Biotechnology 6, 116-122.

Iqbal, M., Bhatti, I.A., Zia-ur-Rehman, M., Bhatti, H.N., Shahid, M., 2014. Efficiency of Advanced Oxidation Processes for Detoxification of Industrial Effluents. Asian Journal of Chemistry 26, 4291-4296.

Iqbal, M., Iqbal, N., Bhatti, I.A., Ahmad, N., Zahid, M., 2016b. Response surface methodology application in optimization of cadmium adsorption by shoe waste: A good option of waste mitigation by waste. Ecological Engineering 88, 265-275.

Iqbal, M., Khera, R.A., 2015. Adsorption of copper and lead in single and binary metal system onto Fumaria indica biomass. Chemistry International 1, 157b-163b.

Iqbal, M., Nisar, J., 2015. Cytotoxicity and mutagenicity evaluation of gamma radiation and hydrogen peroxide treated textile effluents using bioassays. Journal of Environmental Chemical Engineering 3, 1912-1917.

Islam, A., Islam-ud-Din, Iqbal, M., 2015. Application of Response Surface Methodology for the Extraction of Dye from Henna Leaves. Asian Journal of Chemistry 27, 17071710.

Ivanova, E., Staikova, T., Velcheva, I., 2005. Cytogenetic testing of heavy metal and cyanide contaminated river waters in a mining region of Southwest Bulgaria. Journal of Cell and Molecular Biology 4, 00-106.

Ivanova, E., STAIKOVA, T., Velcheva, I., 2008. Cytotoxicity and genotoxicity of heavy metal-and cyanide-contaminated waters in some regions for production and processing of ore in Bulgaria. Bulgarian Journal of Agricultural Science 14, 262-268.

Jadhav, J., Kalyani, D., Telke, A., Phugare, S., Govindwar, S., 2010. Evaluation of the efficacy of a bacterial consortium for the removal of color, reduction of heavy metals, and 
toxicity from textile dye effluent. Bioresource Technology $101,165-173$.

Jadhav, S.B., Chougule, A.S., Shah, D.P., Pereira, C.S., Jadhav, J.P., 2015. Application of response surface methodology for the optimization of textile effluent biodecolorization and its toxicity perspectives using plant toxicity, plasmid nicking assays. Clean Technologies and Environmental Policy 17, 709-720.

Jadhav, S.B., Phugare, S.S., Patil, P.S., Jadhav, J.P., 2011. Biochemical degradation pathway of textile dye Remazol red and subsequent toxicological evaluation by cytotoxicity, genotoxicity and oxidative stress studies. International Biodeterioration \& Biodegradation 65, 733743.

Jadhav, S.B., Surwase, S.N., Kalyani, D.C., Gurav, R.G., Jadhav, J.P., 2012a. Biodecolorization of azo dye Remazol Orange by Pseudomonas aeruginosa $\mathrm{BCH}$ and toxicity (oxidative stress) reduction in Allium cepa root cells. Applied Biochemistry and Biotechnology 168, 1319-1334.

Jadhav, V., Jadhav, A., Chandagade, C., Raut, P., 2012 b. Genotoxicity of bisphenol A on root meristem cells of Allium cepa: A cytogenetic approach. Asian Journal of Water, Environment and Pollution 9, 39-43.

Jafarinejad, S., 2016a. Control and treatment of sulfur compounds specially sulfur oxides (SOx) emissions from the petroleum industry: a review. Chemistry International 2, 242-253.

Jafarinejad, S., 2016b. Odours emission and control in the petroleum refinery: A review. Current Science Perspectives 2, 78-82.

Jamal, M.A., Muneer, M., Iqbal, M., 2015. Photo-degradation of monoazo dye blue 13 using advanced oxidation process. Chemistry International 1, 12-16.

Jeon, C., 2015. Adsorption behavior of silver ions from industrial wastewater onto immobilized crab shell beads. Journal of Industrial and Engineering Chemistry 32, 195200.

Jha, S.K., Nayak, A.K., Sharma, Y.K., 2009. Fluoride toxicity effects in onion (Allium cepa L.) grown in contaminated soils. Chemosphere 76, 353-356.

Jiang, Z., Qin, R., Zhang, H., Zou, J., Shi, Q., Wang, J., Jiang, W., Liu, D., 2014. Determination of $\mathrm{Pb}$ genotoxic effects in Allium cepa root cells by fluorescent probe, microtubular immunofluorescence and comet assay. Plant and Soil 383, 357-372.

Jordan, M.A., Wilson, L., 1998. The use and action of drugs in analyzing mitosis. Methods in Cell Biology 61, 267-295.

Jos, A., Repetto, G., Rios, J., Hazen, M., Molero, M., Del Peso, A., Salguero, M., Fernández-Freire, P., Pérez-Martın, J., Cameán, A., 2003. Ecotoxicological evaluation of carbamazepine using six different model systems with eighteen endpoints. Toxicology in Vitro 17, 525-532.

Jos, A., Repetto, G., Ríos, J.C., del Peso, A., Salguero, M., Hazen, M.J., Molero, M.L., Fernández-Freire, P., Pérez-Martín, J.M., Labrador, V., 2005. Ecotoxicological evaluation of the additive butylated hydroxyanisole using a battery with six model systems and eighteen endpoints. Aquatic Toxicology 71, 183-192.

Jothy, S.L., Chen, Y., Kanwar, J.R., Sasidharan, S., 2013. Evaluation of the Genotoxic Potential against-RadicalMediated DNA Damage and Acute Oral Toxicity of Standardized Extract of Polyalthia longifolia Leaf. Evidence-Based Complementary and Alternative Medicine 2013.

Juchimiuk, J., Maluszynska, J., 2005. Transformed roots of Crepis capillaries a sensitive system for the evaluation of the clastogenicity of abiotic agents. Mutation Research/Genetic Toxicology and Environmental Mutagenesis 565, 129-138.

Júnior, H.M., Da Silva, J., Arenzon, A., Portela, C.S., de Sá Ferreira, I.C.F., Henriques, J.A.P., 2007. Evaluation of genotoxicity and toxicity of water and sediment samples from a Brazilian stream influenced by tannery industries. Chemosphere 67, 1211-1217.

Junior, H.M., Silva, J., Arenzon, A., Portela, C.S., Ferreira, I.C.F.S., Henriques, J.A.P., 2007. Evaluation of genotoxicity and toxicity of water and sediment samples from a Brazilian stream influenced by tannery industries. Chemosphere 67, 1211-1217.

Kadam, A., Dhabbe, R., Kokate, M., Gaikwad, Y., Garadkar, K., 2014. Preparation of $\mathrm{N}$ doped TiO 2 via microwaveassisted method and its photocatalytic activity for degradation of Malathion. Spectrochimica Acta Part A: Molecular and Biomolecular Spectroscopy 133, 669-676.

Kang, R., Qiu, L., Fang, L., Yu, R., Chen, Y., Lu, X., Luo, X., 2016. A novel magnetic and hydrophilic ion-imprinted polymer as a selective sorbent for the removal of cobalt ions from industrial wastewater. Journal of Environmental Chemical Engineering 4, 2268-2277.

Kannangara, D., Pathiratne, A., 2015a. Toxicity assessment of industrial wastewaters reaching Dandugan Oya, Sri Lanka using a plant based bioassay. Journal of the National Science Foundation of Sri Lanka 43, 10.

Kannangara, D., Pathiratne, A., 2015b. Toxicity assessment of industrial wastewaters reaching Dandugan Oya, Sri Lanka using a plant based bioassay. Journal of the National Science Foundation of Sri Lanka 43, 153-163.

Kanu, I., Achi, O., 2011. Industrial effluents and their impact on water quality of receiving rivers in Nigeria. Journal of Applied Technology in Environmental Sanitation 1, 7586.

Kanu, I., Achi, O., Ezeronye, O., Anyanwu, E., 2006. Seasonal variation in bacterial heavy metal biosorption in water samples from Eziama river near soap and brewery industries and the environmental health implications. International Journal of Environmental Science \& Technology 3, 95-102.

Karaismailoglu, M.C., 2014. Investigation of the cytotoxic and genotoxic effects of Artemisia annua methanol extract with the Allium test. Ekoloji 23, 64-74.

Karaismailoglu, M.C., 2015. Investigation of the potential toxic effects of prometryne herbicide on Allium cepa root tip cells with mitotic activity, chromosome aberration, 
micronucleus frequency, nuclear DNA amount and comet assay. Caryologia, 1-7.

Kataeva, M., Kotseruba, V., Terekhina, N., Kutlunina, N., Beljaeva, A., 2012. Allium Root-Micronucleus (AlliumMCN) Test on the Genotoxicity of Soil Samples Contaminated with Heavy Metals. World Applied Sciences Journal 17, 992-1000.

Katnoria, J., Arora, S., Nagpal, A., 2008. Genotoxic potential of agricultural soils of amritsar. Asian Journal of Scientific Research 1, 122-129.

Katnoria, J.K., Arora, S., Bhardwaj, R., Nagpal, A., 2011. Evaluation of genotoxic potential of industrial waste contaminated soil extracts of Amritsar, India. Journal of Environmental Biology/Academy of Environmental Biology, India 32, 363.

Kaymak, F., Muranli, F.G., 2005. The cytogenetic effects of avenoxan on Allium cepa and its relation with pollen sterility. Acta Biologica Hungarica 56, 313-321.

Kern, D.I., de Oliveira Schwaickhardt, R., Lutterbeck, C.A., Kist, L.T., Alcayaga, E.A.L., Machado, E.L., 2015. Ecotoxicological and Genotoxic Assessment of Hospital Laundry Wastewaters. Archives of environmental Contamination and Toxicology 68, 64-73.

Kern, D.I., Schwaickhardt, R.d.O., Mohr, G., Lobo, E.A., Kist, L.T., Machado, E.L., 2013. Toxicity and genotoxicity of hospital laundry wastewaters treated with photocatalytic ozonation. Science of The Total Environment 443, 566-572.

Khan, M.W.A., Ahmad, M., 2006. Detoxification and bioremediation potential of a Pseudomonas fluorescens isolate against the major Indian water pollutants. Journal of Environmental Science and Health Part A 41, 659-674.

Kirsch-Volders, M., Vanhauwaert, A., De Boeck, M., Decordier, I., 2002. Importance of detecting numerical versus structural chromosome aberrations. Mutation Research/Fundamental and Molecular Mechanisms of Mutagenesis 504, 137-148.

Klančnik, K., Drobne, D., Valant, J., Dolenc Koce, J., 2011. Use of a modified Allium test with nanoTiO2. Ecotoxicology and Environmental Safety 74, 85-92.

Klauck, C., Rodrigues, M., Silva, L., 2015. Evaluation of phytotoxicity of municipal landfill leachate before and after biological treatment. Brazilian Journal of Biology 75, 57-62.

Klauck, C.R., Rodrigues, M.A.S., da Silva, L.B., 2013. Toxicological evaluation of landfill leachate using plant (Allium cepa) and fish (Leporinus obtusidens) bioassays. Waste Management \& Research, 0734242X13502388.

Knoll, M.F., Silva, A.C.F., CantoDorow, T.S., Tedesco, S.B., 2006a. Effects of Pterocaulon polystachyum DC.(Asteraceae) on onion (Allium cepa) root-tip cells. Genetics and Molecular Biology 29, 539-542.

Knoll, M.F., Silva, A.C.F.d., Canto-Dorow, T.S.d., Tedesco, S.B., 2006b. Effects of Pterocaulon polystachyum DC.(Asteraceae) on onion (Allium cepa) root-tip cells. Genetics and Molecular Biology 29, 539-542.
Kong, M., Ma, T., 1999. Genotoxicity of contaminated soil and shallow well water detected by plant bioassays. Mutation Research/Fundamental and Molecular Mechanisms of Mutagenesis 426, 221-228.

Konuk, M., Liman, R., Cigerci, I., 2007. Determination of genotoxic effect of boron on Allium cepa root meristematic cells. Pakistan Journal of Botany 39, 73.

Koparal, A.S., Yavuz, Y., Gürel, C., Ögütveren, Ã.B.I., 2007. Electrochemical degradation and toxicity reduction of $\mathrm{CI}$ Basic Red 29 solution and textile wastewater by using diamond anode. Journal of Hazardous Materials 145, 100-108.

Kopliku, D., Mesi, A., 2012a. Correlative Evaluation between Experimental Copper and Lead Ion Concentrations and Root Length of Allium cepa L. in Some Riverside Points of NënShkodra Lowland\#. Journal of International Environmental Application and Science 7, 913.

Kopliku, D., Mesi, A., 2012b. Toxicity Screening of Water Sources in Flooded Agricultural Areas of Nen-Shkodra Lowland Using Allium cepa L. Assay. Journal of Environmental Science and Engineering. A 1, 1197.

Kopliku, D., Mesi, A., Golemi, S., 2012. Citotoxicity and genotoxicity screening of some aquatic bodies in Shkodra region using Allium cepa L. test. Mediterranean Journal of Social Sciences 3, 271-276.

Kovalchuk, O., Kovalchuk, I., Arkhipov, A., Telyuk, P., Hohn, B., Kovalchuk, L., 1998. The Allium cepa chromosome aberration test reliably measures genotoxicity of soils of inhabited areas in the Ukraine contaminated by the Chernobyl accident. Mutation Research/Genetic Toxicology and Environmental Mutagenesis 415, 47-57.

Kuchy, A.H., Wani, A.A., Kamili, A.N., Skinder, B.M., 2015. Pattern of the sensitivity of allium cepa root meristem cells to endosri, nuvan and kvistin pesticides. International Journal of Plant, Animal and Environmental Sciences 5, 218-226.

Kuhn, A.W., Tedesco, M., Boligon, A.A., Athayde, M.L., Laughinghouse, I., Dail, H., Tedesco, S.B., 2015. Chromatographic analysis and antiproliferative potential of aqueous extracts of Punica granatum fruit peels using the Allium cepa test. Brazilian Journal of Pharmaceutical Sciences 51, 241-248.

Kulshrestha, Y., Husain, Q., 2007. Decolorization and degradation of acid dyes mediated by salt fractionated turnip (Brassica rapa) peroxidases. Toxicological \& Environmental Chemistry 89, 255-267.

Kumar, D., Rajeshwari, A., Jadon, P.S., Chaudhuri, G., Mukherjee, A., Chandrasekaran, N., Mukherjee, A., 2015. Cytogenetic studies of chromium (III) oxide nanoparticles on Allium cepa root tip cells. Journal of Environmental Sciences 38, 150-157.

Kumar, S., Kumar, R., Bandopadhyay, A., 2006. Innovative methodologies for the utilisation of wastes from metallurgical and allied industries. Resources, Conservation and Recycling 48, 301-314.

Kumari, A., Sharma, S., Vig, A., 2013. Evaluation of anticytogenetic effects of Brassica oleraceae L. var. Italica 
in Allium cepa L. International Journal of Pharmacy and Pharmaceutical Sciences 4, 120-124.

Kumari, M., Khan, S.S., Pakrashi, S., Mukherjee, A., Chandrasekaran, N., 2011. Cytogenetic and genotoxic effects of zinc oxide nanoparticles on root cells of Allium cepa. Journal of Hazardous Materials 190, 613-621.

Kumari, M., Mukherjee, A., Chandrasekaran, N., 2009a. Genotoxicity of silver nanoparticles in Allium cepa. Science of The Total Environment 407, 5243-5246.

Kumari, M., Mukherjee, A., Chandrasekaran, N., 2009b. Genotoxicity of silver nanoparticles in Allium cepa. Science of the total environment 407, 5243-5246.

Kuraś, M., Nowakowska, J., Śliwińska, E., Pilarski, R., Ilasz, R., Tykarska, T., Zobel, A., Gulewicz, K., 2006. Changes in chromosome structure, mitotic activity and nuclear DNA content from cells of Allium Test induced by bark water extract of Uncaria tomentosa (Willd.) DC. Journal of Ethnopharmacology 107, 211-221.

Kuroda, K., Chosei, T., Nakahara, N., Hatamoto, M., Wakabayashi, T., Kawai, T., Araki, N., Syutsubo, K., Yamaguchi, T., 2015. High organic loading treatment for industrial molasses wastewater and microbial community shifts corresponding to system development. Bioresource Technology 196, 225-234.

Kusumaningrum, H.P., Lunggani, A.T., Nurhakim, M.A., 2012. Chromosomes and Mitotic Cell Division Phase In Onion Roots After 24 Hours Acetoorcein Soaking Time. BIOMA $14,46-48$.

Kwankua, W., Sengsai, S., Kuleung, C., Euawong, N., 2010. Sunlight decreased genotoxicity of azadirachtin on root tip cells of Allium cepa and Eucrosia bicolor. Ecotoxicology and Environmental Safety 73, 949-954.

Kwasniewska, J., NaLecz-Jawecki, G., Skrzypczak, A., PLaza, G., Matejczyk, M., 2012. An assessment of the genotoxic effects of landfill leachates using bacterial and plant tests. Ecotoxicology and Environmental Safety 75(1), 55-62.

Lateef, A., 2004. The microbiology of a pharmaceutical effluent and its public health implications. World Journal of Microbiology and Biotechnology 20, 167-171.

Lateef, A., Ufuoma, P., Yekeen, T., 2007. Bacteriology and genotoxicity of some pharmaceutical wastewaters in Nigeria. International Journal of Environment and Health 1, 551-562.

Laughinghouse Iv, H.D., Prá, D., Silva-Stenico, M.E., Rieger, A., Frescura, V.D.-S., Fiore, M.F., Tedesco, S.B., 2012. Biomonitoring genotoxicity and cytotoxicity of Microcystis aeruginosa (Chroococcales, Cyanobacteria) using the Allium cepa test. Science of The Total Environment 432, 180-188.

Lee, C.-G., Song, M.-K., Ryu, J.-C., Park, C., Choi, J.-W., Lee, S.-H., 2016. Application of carbon foam for heavy metal removal from industrial plating wastewater and toxicity evaluation of the adsorbent. Chemosphere 153, 1-9.

Lee, J., Pandey, B.D., 2011. Bio-processing of solid wastes and secondary resources for metal extraction-A review. Waste Management 32, 3-18.
Lee, M.-T., Li, C.-W., Liu, J.-C., 2015. Recovery of fluoride as perovskite-like minerals from industrial wastewater. Separation and Purification Technology 156, Part 3, 1057-1063.

Leite, A.d.S., Dantas, A.F., Oliveira, G.L.d.S., Gomes Júnior, A.L., de Lima, S.G., Citó, A.M.d.G.L., de Freitas, R.M., MeloCavalcante, A.A.d.C., Dantas Lopes, J.A., 2015. Evaluation of toxic, cytotoxic, mutagenic, and antimutagenic activities of natural and technical cashew nut shell liquids using the Allium cepa and Artemia salina bioassays. BioMed Research International 2015, 10.1155/2015/626835.

Leme, D.M., Angelis, D.F., Marin-Morales, M.A., 2008. Action mechanisms of petroleum hydrocarbons present in waters impacted by an oil spill on the genetic material of Allium cepa root cells. Aquatic Toxicology 88, 214-219.

Leme, D.M., Marin-Morales, M.A., 2008. Chromosome aberration and micronucleus frequencies in Allium cepa cells exposed to petroleum polluted water--A case study. Mutation Research/Genetic Toxicology and Environmental Mutagenesis 650, 80-86.

Leme, D.M., Marin-Morales, M.A., 2009. Allium cepa test in environmental monitoring: A review on its application. Mutation Research/Reviews in Mutation Research 682, 71-81.

Lerda, D., 1992. The effect of lead on Allium cepa L. Mutation Research Letters 281, 89-92.

Lerda, D., Biagi Bistoni, M., Pelliccioni, P., Litterio, N., 2010. Allium cepa as a biomonitor of ochratoxin A toxicity and genotoxicity. Plant Biology 12, 685-688.

Levan, A., 1938. The effect of colchicine on root mitoses in Allium. Hereditas 24, 471-486.

Liman, R., 2013. Genotoxic effects of Bismuth (III) oxide nanoparticles by Allium and Comet assay. Chemosphere 93, 269-273.

Liman, R., AkyÄ \pm l, D., Eren, Y., Konuk, M., 2010a. Testing of the mutagenicity and genotoxicity of metolcarb by using both Ames/Salmonella and Allium test. Chemosphere 80, 1056-1061.

Liman, R., Ciğerci, İ.H., Akyıl, D., Eren, Y., Konuk, M., 2011. Determination of genotoxicity of Fenaminosulf by Allium and Comet tests. Pesticide Biochemistry and Physiology 99, 61-64.

Liman, R., Ciğerci, İ.H., Öztürk, N.S., 2015. Determination of genotoxic effects of Imazethapyr herbicide in Allium cepa root cells by mitotic activity, chromosome aberration, and comet assay. Pesticide Biochemistry and Physiology $118,38-42$.

Liu, D., Jiang, W., Gao, X., 2003. Effects of cadmium on root growth, cell division and nucleoli in root tip cells of garlic. Biologia Plantarum 47, 79-83.

Liu, D., Jiang, W., Wang, W., Zhao, F., Lu, C., 1994. Effects of lead on root growth, cell division, and nucleolus of Allium cepa. Environmental Pollution 86, 1-4.

Liu, J., Huang, K., Xie, K., Yang, Y., Liu, H., 2016. An ecological new approach for treating $\mathrm{Cr}(\mathrm{VI})$-containing industrial 
wastewater: Photochemical reduction. Water Research 93, 187-194.

LIU, X.-d., YI, H.-l., LI, H.-h., 2009. Genotoxicity of potassium cyanide in Vicia faba and AUium cepa root tips. Journal of Safety and Environment 2, 002.

Lovatel, R.H., Neves, R.M., Oliveira, G.R., Mauler, R.S., Crespo, J.S., Carli, L.N., Giovanela, M., 2015. Disinfection of biologically treated industrial wastewater using montmorillonite/alginate/nanosilver hybrids. Journal of Water Process Engineering 7, 273-279.

Lubini, G., Fachinetto, J., Laughinghouse, H., Paranhos, J., Silva, A., Tedesco, S., 2008. Extracts affecting mitotic division in root-tip meristematic cells. Biologia 63, 647-651.

Lutterbeck, C.A., Kern, D.I., Machado, Ê.L., Kümmerer, K., 2015. Evaluation of the toxic effects of four anti-cancer drugs in plant bioassays and its potency for screening in the context of waste water reuse for irrigation. Chemosphere 135, 403-410.

Ma, T.H., Xu, Z., Xu, C., McConnell, H., Valtierra Rabago, E., Adriana Arreola, G., Zhang, H., 1995. The improved Allium/Vicia root tip micronucleus assay for clastogenicity of environmental pollutants. Mutation Research/Environmental Mutagenesis and Related Subjects 334, 185-195.

Machado, E.L., de Sales Dambros, V., Kist, L.T., Lobo, E.A.A., Tedesco, S.B., Moro, C.C., 2012. Use of ozonization for the treatment of dye wastewaters containing Rhodamine B in the agate industry. Water, Air, \& Soil Pollution 223, 1753-1764.

Magdaleno, A., Juárez, Á.B., Dragani, V., Saenz, M.E., Paz, M., Moretton, J., 2014. Ecotoxicological and genotoxic evaluation of Buenos Aires city (Argentina) hospital wastewater. Journal of Toxicology 2014, 1-10.

Magdaleno, A., Mendelson, A., de Iorio, A.F., Rendina, A., Moretton, J., 2008. Genotoxicity of leachates from highly polluted lowland river sediments destined for disposal in landfill. Waste Management 28, 2134-2139.

Mahmood, A., Malik, R.N., 2014. Human health risk assessment of heavy metals via consumption of contaminated vegetables collected from different irrigation sources in Lahore, Pakistan. Arabian Journal of Chemistry 7, 91-99.

Maiti, S., Sasmal, K., Sinha, S.S., Singh, M., 2016. Analysis of cytotoxicity and genotoxicity on E. coli, human blood cells and Allium cepa suggests a greater toxic potential of hair dye. Ecotoxicology and Environmental Safety 124, 248-254.

Majewska, A., Wolska, E., Śliwińska, E., Furmanowa, M., Urbańska, N., Pietrosiuk, A., Zobel, A., Kuraś, M., 2003. Antimitotic effect, G2/M accumulation, chromosomal and ultrastructure changes in meristematic cells of Allium cepa L. root tips treated with the extract from Rhodiola rosea roots. Caryologia 56, 337-351.

Majolagbe, A.O., Adeyi, A.A., Osibanjo, O., 2016a. Vulnerability assessment of groundwater pollution in the vicinity of an active dumpsite (Olusosun), Lagos, Nigeria. Chemistry International 2, 232-241.
Majolagbe, A.O., Adeyi, A.A., Osibanjo, 0., 2016b. Vulnerability assessment of groundwater pollution in the vicinity of an active dumpsite (Olusosun), Lagos, Nigeria. Chemistry International 2, 232-241.

Manzoor, Q., Nadeem, R., Iqbal, M., Saeed, R., Ansari, T.M., 2013. Organic acids pretreatment effect on Rosa bourbonia phyto-biomass for removal of $\mathrm{Pb}(\mathrm{II})$ and $\mathrm{Cu}(\mathrm{II})$ from aqueous media. Bioresource Technology 132, 446-452.

Marcano, L., Carruyo, I., Del Campo, A., Montiel, X., 2002. Effect of Cadmium on the Nucleoli of Meristematic Cells of Onion Allium cepa L: An Ultrastructural Study. Environmental Research 88, 30-35.

Marcano, L., Carruyo, I., Del Campo, A., Montiel, X., 2004. Cytotoxicity and mode of action of maleic hydrazide in root tips of Allium cepa L. Environmental research 94, 221-226.

Marcano, L., Carruyo, I., Fernandez, Y., Montiel, X., Torrealba, Z., 2006. Determination of vanadium accumulation in onion root cells (Allium cep a L.) and its correlation with toxicity. Biocell 30, 259-267.

Marin-Morales, M.A., 2015. Micronuclei and chromosome aberrations derived from the action of Atrazine herbicide in Allium cepa meristematic cells. SDRP Journal of Earth Sciences \& Environmental Studies 1, 7.

Maselli, B.d.S., Luna, L.A., Palmeira, J.d.O., Tavares, K.P., Barbosa, S., Beijo, L.A., Umbuzeiro, G.A., Kummrow, F., 2015. Ecotoxicity of raw and treated effluents generated by a veterinary pharmaceutical company: a comparison of the sensitivities of different standardized tests. Ecotoxicology 24, 795-804.

Masood, F., Malik, A., 2013a. Cytotoxic and genotoxic potential of tannery waste contaminated soils. Science of The Total Environment 444, 153-160.

Masood, F., Malik, A., 2013b. Mutagenicity and genotoxicity assessment of industrial wastewaters. Environmental Science and Pollution Research 20, 7386-7397.

Matsumoto, S.T., Mantovani, M.S., Malaguttii, M.I.A., Dias, A.L., Fonseca, I.C., Marin-Morales, M.A., 2006. Genotoxicity and mutagenicity of water contaminated with tannery effluents, as evaluated by the micronucleus test and comet assay using the fish Oreochromis niloticus and chromosome aberrations in onion root-tips. Genetics and Molecular Biology 29, 148-158.

Matsumoto, S.T., Marin-Morales, M.A., 2004. Mutagenic potential evaluation of the water of a river that receives tannery effluent using the Allium cepa test system. Cytologia 69, 399-408.

Mauro, M., Pesarini, J., Marin-Morales, M., Monreal, M., Monreal, A., Mantovani, M., Oliveira, R., 2014. Evaluation of the antimutagenic activity and mode of action of the fructooligosaccharide inulin in the meristematic cells of Allium cepa culture. Genetics and Molecular Research 13, 4808-4819.

Mazzeo, D.E.C., Fernandes, T.C.C., Levy, C.E., Fontanetti, C.S., Marin-Morales, M.A., 2015. Monitoring the natural 
attenuation of a sewage sludge toxicity using the Allium cepa test. Ecological Indicators 56, 60-69.

Mazzeo, D.E.C., Fernandes, T.C.C., Marin-Morales, M.A., 2011 a. Cellular damages in the Allium cepa test system, caused by BTEX mixture prior and after biodegradation process. Chemosphere 85(1), 13-18.

Mazzeo, D.E.C., Marin-Morales, M.A., 2015. Genotoxicity evaluation of environmental pollutants using analysis of nucleolar alterations. Environmental Science and Pollution Research 22, 9796-9806.

Md Nazmul, H., Akhtaruzzaman, M., Md Zakir, S., 2013. Estimation of Vitamins B-Complex (B 2, B 3, B 5 and B 6) of Some Leafy Vegetables Indigenous to Bangladesh by HPLC Method. Journal of Analytical Sciences, Methods and Instrumentation 3, 24-29.

Meier, J.R., Chang, L.W., Jacobs, S., Torsella, J., Meckes, M.C., Smith, M.K., 1997. Use of plant and earthworm bioassays to evaluate remediation of soil from a site contaminated with polychlorinated biphenyls. Environmental Toxicology and Chemistry 16, 928-938.

Mekki, L., 2014. Genoprotectivity of methanol and ethanol extracted leaf sap of Trigonella foenum-graecum in Allium cepa root assay. Acta Biologica Hungarica 65, 8595.

Mellado-García, P., Puerto, M., Prieto, A.I., Pichardo, S., Martín-Cameán, A., Moyano, R., Blanco, A., Cameán, A.M., 2016. Genotoxicity of a thiosulfonate compound derived from Allium sp. intended to be used in active food packaging: In vivo comet assay and micronucleus test. Mutation Research/Genetic Toxicology and Environmental Mutagenesis 800-801, 1-11.

Melnick, R.L., Dunnick, J.K., Sandler, D.P., Elwell, M.R., Barrett, J.C., 1994. Trihalomethanes and Other Environmental Factors That Contribute to Colorectal Cancer. Environmental Health Perspectives 102, 586.

Mendes, S.S., Andrade, J.A., Xavier, M.A., Secundo Junior, J.A., Pantaleão, S.M., Estevam, C.S., Garcia, C.A., Ferrari, S.F., 2012. Genotoxicity test of Maytenus rigida and Aristolochia birostris in the radicular meristem of the onion, Allium cepa. Revista Brasileira de Farmacognosia 22, 76-81.

Meneses-Jácome, A., Diaz-Chavez, R., Velásquez-Arredondo, H.I., Cárdenas-Chávez, D.L., Parra, R., Ruiz-Colorado, A.A., 2016. Sustainable Energy from agro-industrial wastewaters in Latin-America. Renewable and Sustainable Energy Reviews 56, 1249-1262.

Meng, Z., Zhang, L., 1992. Cytogenetic damage induced in human lymphocytes by sodium bisulfite. Mutation Research/Genetic Toxicology 298, 63-69.

Mesi, A., Kopliku, D., 2011. Allium Test of Root Growth for Toxicity Assessment as a Standard in Environmental Monitoring of Some Aqueous Sources of Malësia e Madhe $^{\wedge}$ sup\#. Journal of International Environmental Application and Science 6, 654.

Mesi, A., Kopliku, D., 2012. Toxicity Evaluation of Leachates from some Urban Wastewater Collectors of Shkodra using Allium cepa L. Test. Journal of International Environmental Application and Science 7, 907-912.

Mesi, A., Kopliku, D., 2013. Cytotoxic and Genotoxic Potency Screening of Two Pesticides on Allium cepa L. Procedia Technology 8, 19-26.

Mesi, A.D., Kopliku, D., 2014. Toxic potency evaluation of metal-doped river water $(\mathrm{Cr}, \mathrm{Cu}$ and $\mathrm{Pb})$, on Allium cepa L. An Albanian case. Athens Journal of Sciences 1, 255267.

Mishra, K., 1993. Cytotoxic effects of distillary waste on Allium cepa L. Bulletin of environmental contamination and toxicology 50, 199-204.

Mishra, K., Sanwal, E., Tandon, P., Gupta, K., 2015. Influence of pesticide effluent on Allium cepa L.(onion) plants. International Journal of Environment 4, 95-105.

Misik, M., Knasmueller, S., Ferk, F., Cichna-Markl, M., Grummt, T., Schaar, H., Kreuzinger, N., 2011. Impact of ozonation on the genotoxic activity of tertiary treated municipal wastewater. Water Research 45(12), 3681-91.

Mišík, M., Pichler, C., Rainer, B., Filipic, M., Nersesyan, A., Knasmueller, S., 2014. Acute toxic and genotoxic activities of widely used cytostatic drugs in higher plants: possible impact on the environment. Environmental Research 135, 196-203.

Mohammed, K.P., Aarey, A., Tamkeen, S., Jahan, P., 2015. Forskolin: Genotoxicity assessment in Allium cepa. Mutation Research/Genetic Toxicology and Environmental Mutagenesis 777, 29-32.

Mohan Murali Achary, V., Jena, S., Panda, K.K., Panda, B.B., 2008. Aluminium induced oxidative stress and DNA damage in root cells of Allium cepa L. Ecotoxicology and environmental safety 70, 300-310.

Monarca, S., Feretti, D., Collivignarelli, C., Guzzella, L., Zerbini, I., Bertanza, G., Pedrazzani, R., 2000. The influence of different disinfectants on mutagenicity and toxicity of urban wastewater. Water Research 34, 4261-4269.

Monarca, S., Rizzoni, M., Gustavino, B., Zani, C., Alberti, A., Feretti, D., Zerbini, I., 2003. Genotoxicity of surface water treated with different disinfectants using in situ plant tests. Environmental and Molecular Mutagenesis 41, 353-359.

Mondrala, S., Eastmond, D.A., 2010. Topoisomerase II inhibition by the bioactivated benzene metabolite hydroquinone involves multiple mechanisms. Chemicobiological Interactions 184, 259-268.

Mosuro, A., Bakare, A., Koofreh, M., Ngenwi, A., 1999. Genetic safety evaluation of pesticides using Allium cepa assay. Cytology and Genetics 34, 173-182.

Moudgal, C., Lipscomb, J., Bruce, R., 2000. Potential health effects of drinking water disinfection by-products using quantitative structure toxicity relationship. Toxicology 147, 109-131.

Mumtaz, M.W., Mukhtar, H., Dilawer, U.A., Hussain, S.M., Hussain, M., Iqbal, M., Adnan, A., Nisar, J., 2016. Biocatalytic transesterification of Eruca sativa oil for the production of biodiesel. Biocatalysis and Agricultural Biotechnology 5, 162-167. 
Muneer, M., Adeel, S., Ayub, S., Zuber, M., Ur-Rehman, F., Kanjal, M., Iqbal, M., Kamran, M., 2016. Dyeing behaviour of microwave assisted surface modified polyester fabric using disperse orange 25: improvement in colour strength and fastness properties. Oxidation Communications 39, 1430-1439.

Musanovic, J., Ramic, N., Nefic, H., Dzubur, A., 2013. Chromosome aberration and irregular cell cycle in Allium cepa root cells caused by different concentrations of Alprazolam. International Journal of Collaborative Research on Internal Medicine \& Public Health 5, 407418.

Mushtaq, M., Bhatti, H.N., Iqbal, M., Noreen, S., 2016. Eriobotrya japonica seed biocomposite efficiency for copper adsorption: Isotherms, kinetics, thermodynamic and desorption studies. Journal of Environmental Management 176, 21-33.

Mustafa, Y., Suna Arikan, E., 2008. Genotoxicity testing of quizalofop-P-ethyl herbicide using the Allium cepa anaphase-telophase chromosome aberration assay. Caryologia 61, 45-52.

Nadaf, N.H., Ghosh, J.S., 2014. Biodegradation of a Paper and Pulp Effluent by Rhodococcus sp. NCIM 2891 and Its Toxicity Assessments. Clean-Soil, Air, Water 42, 456-461.

Nadeem, R., Manzoor, Q., Iqbal, M., Nisar, J., 2016 a. Biosorption of $\mathrm{Pb}$ (II) onto immobilized and native Mangifera indica waste biomass. Journal of Industrial and Engineering Chemistry 35, 184-195.

Nagaonkar, D., Shende, S., Rai, M., 2015. Biosynthesis of copper nanoparticles and its effect on actively dividing cells of mitosis in Allium cepa. Biotechnology progress 31, 557-565.

Nagarajan, N., Gunasekaran, P., Rajendran, P., 2014. Impacts of electroplating industrial effluents on plants, potable water and genotoxicity to meristamatic cells of onion root tips. The Scitech Journal 1, 25-31.

Nantes, C., Pesarini, J., Mauro, M., Monreal, A., Ramires, A., Oliveira, R., 2014. Evaluation of the antimutagenic activity and mode of action of carrageenan fiber in cultured meristematic cells of Allium cepa. Genetics and Molecular Research 13, 9523.

Netto, E., Madeira, R., Silveira, F., Fiori, M., Angioleto, E., Pich, C., Geremias, R., 2013. Evaluation of the toxic and genotoxic potential of acid mine drainage using physicochemical parameters and bioassays. Environmental Toxicology and Pharmacology 35, 511516.

Nielsen, M., Rank, J., 1994. Screening of toxicity and genotoxicity in wastewater by the use of the use of the Allium test. Hereditas 121, 249-254.

Nisar, J., Razaq, R., Farooq, M., Iqbal, M., Khan, R.A., Sayed, M., Shah, A., Rahman, I.u., 2017. Enhanced biodiesel production from Jatropha oil using calcined waste animal bones as catalyst. Renewable Energy 101, 111-119.

Nisar, J., Sayed, M., Khan, F.U., Khan, H.M., Iqbal, M., Khan, R.A., Anas, M., 2016. Gamma - irradiation induced degradation of diclofenac in aqueous solution: Kinetics, role of reactive species and influence of natural water parameters. Journal of Environmental Chemical Engineering 4, 2573-2584.

Njoku, K., Akinola, M., Tommy, I., 2015. Genotoxicity of Industrial Paint Effluent on the Root Meristem of Allium Cepa. IOSR Journal of Environmental Science, Toxicology and Food Technology 9, 11-17.

Nunes, E.A., De Lemos, C.T., Gavronski, L., Moreira, T.N., Oliveira, N.C.D., Da Silva, J., 2011. Genotoxic assessment on river water using different biological systems. Chemosphere 84, 47-53.

Obek, E., Sasmaz, A., 2011. Bioaccumulation of aluminum by Lemna gibba L. from secondary treated municipal wastewater effluents. Bulletin of Environmental Contamination and Toxicology 86, 217-220.

Oberholster, P., Botha, A.-M., Cloete, T., 2008. Biological and chemical evaluation of sewage water pollution in the Rietvlei nature reserve wetland area, South Africa. Environmental Pollution 156, 184-192.

Obidoska, G., Jasińska, D., 2008. Phytotoxicity and potential genotoxicity of Radiowo municipal landfill leachate. Annals of Warsaw University of Life Sciences-SGGW. Land Reclamation 40, 39-44.

Obidoska, G., Lichmira, K., 2013. Assessment of the toxicity and genotoxicity of Vistula river water at selected points in Warsaw using Allium test. Annals of Warsaw University of Life Sciences-SGGW. Horticulture and Landscape Architecture 34, 15-20.

Obute, G., Ekeke, C., Izuka, D., 2016. Genotoxicity Assessment of Refined Petroleum Products and Popular Local Soft drink (Zobo) in Daily Use in Nigeria. Research Journal of Mutagenesis 6, 22.

Obute, G.C., Osuji, L.C., Kalio, C., 2005. Genotoxicity of petroleum refinery waste water in Nigeria. Global Journal of Environmental Sciences 3, 55-58.

Odeigah, P., Nurudeen, O., Amund, O., 1997. Genotoxicity of oil field wastewater in Nigeria. Hereditas 126, 161-167.

Ohe, T., Watanabe, T., Wakabayashi, K., 2004. Mutagens in surface waters: a review. Mutation Research/Reviews in Mutation Research 567, 109-149.

Oliveira-Martins, C.R., Grisolia, C.K., 2009. Toxicity and genotoxicity of wastewater from gasoline stations. Genetics and Molecular Biology 32, 853-856.

Olorunfemi, D., Akpaja, E., Agbi, B., 2011a. Cytological and toxicological properties of a medicinal mushroom. Nigerian Journal of Pharmaceutical and Applied Science Research 1, 36-43.

Olorunfemi, D., Duru, E., Okieimen, F., 2012. Induction of chromosome aberrations in Allium cepa L. root tips on exposure to ballast water. Caryologia 65, 147-151.

Olorunfemi, D., Ogieseri, U., Akinboro, A., 2011b. Genotoxicity Screening of Industrial Effluents using Onion bulbs (Allium cepa L.). Journal of Applied Sciences and Environmental Management 15, 6.

Olorunfemi, D., Okieimen, E., Muokebe, I., 2015a. Evaluation of bilge water-induced plant genotoxicity using randomly 
amplified polymorphic dna (RAPD). Ife Journal of Science $17,121-129$.

Olorunfemi, D., Okoloko, G., Bakare, A., Akinboro, A., 2011c. Cytotoxic and genotoxic effects of cassava effluents using the Allium cepa assay. Research Journal of Mutagenesis 1, $1-9$.

Olorunfemi, D., Olomukoro, J., Anani, O., 2015b. Toxicity Evaluation and cytogenetic screening of process water using a plant bioassay. Nigerian Journal of Basic and Applied Sciences 23, 31-37.

Olorunfemi, D., Olorunfemi, O., Agbozu, I., 2014a. Genotoxicity assessment of contaminated drinking water sources in a rural community in Edo State of Nigeria. Journal of Geoscience and Environment Protection 2, 52.

Olorunfemi, D., Omo-Ataga, S., Ofomata, R., 2013. Studies on the physicochemical properties and genotoxicity of effluents from a dairy industry. Bayero Journal of Pure and Applied Sciences 5, 11-18.

Olorunfemi, D.I., 2013. Cytogenotoxicity assessment of a University borehole water supply using the Allium cepa test. Journal of Scientific Research and Development 14, 623-627.

Olorunfemi, D.I., Duru, L., Olorunfemi, O.P., 2015c. Genotoxic effects of bilge water on mitotic activity in Allium cepa L. Caryologia https://doi.org/10.1080/00087114.2015.1032574

Olorunfemi, D.I., Ehwre, E.O., 2011. Chromosomal Aberrations Induced in Root Tips of Allium cepa by Squeezed Garri Extracts. Report and Opinion 2, 166-171.

Olorunfemi, D.I., Okieimen, E.A., Ovwemuvwose, J., 2014 b. DNA integrity of onion (Allium cepa L.) root cells exposed to ballast water. Studia Universitatis" Vasile Goldis" Arad. Seria Stiintele Vietii (Life Sciences Series) 24, 305.

Onwuamah, C.K., Ekama, S.O., Audu, R.A., Ezechi, O.C., Poirier, M.C., Odeigah, P.G.C., 2014. Exposure of Allium cepa root cells to zidovudine or Nevirapine induces cytogenotoxic changes. PloS one 9, e90296.

Onyemaobi, O., Williams, G., Adekoya, K., 2012. Cytogenetic effects of two food preservatives, sodium metabisulphite and sodium benzoate on the root tips of Allium cepa Linn. Ife Journal of Science 14, 155.

Oriaku, V.A., Otubanjo, O.A., Aderemi, A.O., Otitoloju, A.A., 2011. Genotoxic endpoints in Allium cepa and clarias gariepinus exposed to textile effluent. International Journal of Environmental Protection 1, 48-52.

Oyeyemi, I.T., Bakare, A.A., 2013. Genotoxic and antigenotoxic effect of aqueous extracts of Spondias mombin L., Nymphea lotus L. and Luffa cylindrica L. on Allium cepa root tip cells. Caryologia 66, 360-367.

Ozakca, D.U., Silah, H., 2013. Genotoxicity effects of Flusilazole on the somatic cells of Allium cepa. Pesticide Biochemistry and Physiology 107, 38-43.

Özkara, A., Akyıl, D., Eren, Y., Erdoğmuş, S.F., 2015a. Potential cytotoxic effect of Anilofos by using Allium cepa assay. Cytotechnology 67, 783-791.

Özkara, A., Akyll, D., Eren, Y., Erdoğmuş, S.F., Konuk, M., Sağlam, E., 2015b. Assessment of cytotoxic and genotoxic potential of pyracarbolid by Allium test and micronucleus assay. Drug and Chemical Toxicology 38, 337-341.

Paiva, T., Garcias, G., Martino-Roth, M., 2008. Increasing mutagenicity of São Gonçalo Channel waters based on the Allium cepa test. Genetics and Molecular Research 8, 299-309.

Pakrashi, S., Jain, N., Dalai, S., Jayakumar, J., Chandrasekaran, P.T., Raichur, A.M., Chandrasekaran, N., Mukherjee, A., 2014. In vivo genotoxicity assessment of titanium dioxide nanoparticles by Allium cepa root tip assay at high exposure concentrations. PloS one 9, e87789.

Pal, R., Nandi, S., 1990. Cytological abnormalities induced by mercury water pollutants on Allium cepa L. Bulletin of Environmental Contamination and Toxicology 45, 767774.

Palacio, S.M., Espinoza-Quiñones, F.R., Galante, R.M., Zenatti, D.C., Seolatto, A.A., Lorenz, E.K., Zacarkim, C.E., Rossi, N., Rizzutto, M.d.A., Tabacniks, M.H., 2005. Correlation between heavy metal ions (Copper, Zinc, Lead) concentrations and root length of Allium cepa L. in polluted river water. Brazilian Archives of Biology and Technology 48, 191-196.

Palma, P., Alvarenga, P., Palma, V., Matos, C., Fernandes, R.M., Soares, A., Barbosa, I.R., 2010. Evaluation of surface water quality using an ecotoxicological approach: a case study of the Alqueva Reservoir (Portugal). Environmental Science and Pollution Research 17, 703716.

Palma, P., Köck-Schulmeyer, M., Alvarenga, P., Ledo, L., Barbosa, I., De Alda, M.L., Barceló, D., 2014. Risk assessment of pesticides detected in surface water of the Alqueva reservoir (Guadiana basin, southern of Portugal). Science of The Total Environment 488, 208219.

Panda, K.K., Patra, J., Panda, B.B., 1997. Persistence of cadmium-induced adaptive response to genotixicity of maleic hydrazide and methyl mercuric chloride in root meristem cells of Allium cepa L.: Differential inhibition by cycloheximide and buthionine sulfoximine. Mutation Research/Genetic Toxicology and Environmental Mutagenesis 389, 129-139.

Pandey, B.K., Vyas, S., Pandey, M., Gaur, A., 2016 a. Characterisation of municipal solid waste generated from Bhopal, India. Current Science Perspectives 2, 52-56.

Pandey, B.K., Vyas, S., Pandey, M., Gaur, A., 2016b. Municipal solid waste to energy conversion methodology as physical, thermal, and biological methods. Current Science Perspectives 2, 39-46.

Pandey, H., Kumar, V., Roy, B.K., 2014. Assessment of genotoxicity of some common food preservatives using Allium cepa L. as a test plant. Toxicology Reports 1, 300308.

Panneerselvam, N., Palanikumar, L., Gopinathan, S., 2012. Chromosomal aberrations induced by Glycidol in Allium cepa L. root meristem cells. International Journal of Pharma Sciences and Research 3, 300-304. 
Papa, M., Ceretti, E., Viola, G.C.V., Feretti, D., Zerbini, I., Mazzoleni, G., Steimberg, N., Pedrazzani, R., Bertanza, G., 2016. The assessment of WWTP performance: Towards a jigsaw puzzle evaluation? Chemosphere 145, 291-300.

Pastori, T., Flores, F.C., Boligon, A.A., Athayde, M.L., da Silva, C.d.B., do Canto-Dorow, T.S., Tedesco, S.B., 2013. Genotoxic effects of Campomanesia xanthocarpa extracts on Allium cepa vegetal system. Pharmaceutical Biology 51, 1249-1255.

Patel, T.L., Patel, B.C., Kadam, A.A., Tipre, D.R., Dave, S.R., 2015. Application of novel consortium TSR for treatment of industrial dye manufacturing effluent with concurrent removal of ADMI, COD, heavy metals and toxicity. Water Science and Technology 71, 1293-1300.

Pathiratne, A., Hemachandra, C.K., De Silva, N., 2015. Efficacy of Allium cepa test system for screening cytotoxicity and genotoxicity of industrial effluents originated from different industrial activities. Environmental Monitoring and Assessment 187, 1-12.

Patil, A.V., Jadhav, J.P., 2013. Evaluation of phytoremediation potential of Tagetes patula L. for the degradation of textile dye Reactive Blue 160 and assessment of the toxicity of degraded metabolites by cytogenotoxicity. Chemosphere 92, 225-232.

Patnaik, A.R., Achary, V.M.M., Panda, B.B., 2013. Chromium (VI)-induced hormesis and genotoxicity are mediated through oxidative stress in root cells of Allium cepa L. Plant growth regulation 71, 157-170.

Patra, J., Sahoo, M.K., Panda, B.B., 2005. Salicylic acid triggers genotoxic adaptation to methyl mercuric chloride and ethyl methane sulfonate, but not to maleic hydrazide in root meristem cells of Allium cepa L. Mutation Research/Genetic Toxicology and Environmental Mutagenesis 581, 173-180.

Paul, A., Nag, S., Sinha, K., 2013. Cytological effects of blitox on root mitosis of Allium cepa. International Journal of Scientific and Research 3, 1-7.

Pavlica, M., Besendorfer, V., Rosa, J., Papes, D., 2000. The cytotoxic effect of wastewater from the phosphoric gypsum depot on common oak (Quercus robur L.) and shallot (Allium cepa var. ascalonicum). Chemosphere 41, 1519-1527.

Paz, M., Muzio, H., Mendelson, A., Magdaleno, A., Tornello, C., Balbis, N., Moretton, J., 2006. Evaluation of genotoxicity and toxicity of Buenos Aires city hospital wastewater samples. Journal of the Brazilian Society of Ecotoxicology 1, 1-6.

Pedrazzani, R., Ceretti, E., Zerbini, I., Casale, R., Gozio, E., Bertanza, G., Gelatti, U., Donato, F., Feretti, D., 2012. Biodegradability, toxicity and mutagenicity of detergents: Integrated experimental evaluations. Ecotoxicology and Environmental Safety 84, 274-281.

Pedro-Escher, J., Christofoletti, C.A., Ansoar-Rodríguez, Y., Fontanetti, C.S., 2016. Sugarcane vinasse, a residue of ethanol industry: toxic, cytotoxic and genotoxic potential using the Allium cepa test. Journal of Environmental Protection 7, 602.
Pekol, S., 2014. Ecotoxicological assessment of metalworking fluids using the Allium cepa test procedure. Chemistry and Ecology 30, 66-75.

Pekol, S., Caglar, A., Aydinli, B., 2012. The toxic and environmental evaluation of pyrolytic liquids by Allium cepa test. Chemistry and Ecology 28, 65-73.

Pelegrini, N.N.d.B., Pelegrini, R.T., Paterniani, J.E.S., 2007. Ecotoxicological evaluation of leachate from the Limeira sanitary landfill with a view to identifying acute toxicity. Ambiente \& Água-An Interdisciplinary Journal of Applied Science 2, 34-43.

Pepper, I., Galanti, N., Sans, J., Lopez-Saez, J.F., 1988. Reversible inhibition of root growth and cell proliferation by pentavalent arsenic in Allium cepa L. Environmental and Experimental Botany 28, 9-18.

Pereyra, M.T., Fernández Band, B.S., Lista, A.G., 2015. Measurement uncertainty estimated from accuracy assessment by using an in-house reference material: two case studies for the extraction of total PAH in industrial wastewater sludges. Microchemical Journal 119, 145151.

Pervaiz, M., Butt, K.M., Raza, M.A., Rasheed, A., Ahmad, S., Adnan, A., Iqbal, M., 2015. Extraction and applications of aluminum hydroxide from bauxite for commercial consumption. Chemistry International 1, 99-102.

Pesnya, D.S., 2013. Cytogenetic effects of chitosan-capped silver nanoparticles in the Allium cepa test. Caryologia: International Journal of Cytology, Cytosystematics and Cytogenetics 66, 275-281.

Peter, U.C., Chinedu, U., 2016a. Model prediction for constant area, variable pressure drop in orifice plate characteristics in flow system. Chemistry International 2, 80-88.

Peter, U.C., Chinedu, U., 2016b. Model prediction for constant area, variable pressure drop in orifice plate characteristics in flow system. Chemistry International 2, 80-88.

Petriccione, M., Forte, V., Valente, D., Ciniglia, C., 2013. DNA integrity of onion root cells under catechol influence. Environmental Science and Pollution Research 20, 48594871.

Phugare, S., Patil, P., Govindwar, S., Jadhav, J., 2010. Exploitation of yeast biomass generated as a waste product of distillery industry for remediation of textile industry effluent. International Biodeterioration \& Biodegradation 64, 716-726.

Phugare, S.S., Kalyani, D.C., Surwase, S.N., Jadhav, J.P., 2011. Ecofriendly degradation, decolorization and detoxification of textile effluent by a developed bacterial consortium. Ecotoxicology and Environmental Safety 74, 1288-1296.

Pierantozzi, P., Zampini, C., Torres, M., Isla, M.I., Verdenelli, R.A., Meriles, J.M., Maestri, D., 2012. Physico-chemical and toxicological assessment of liquid wastes from olive processing? related industries. Journal of the Science of Food and Agriculture 30, 216-23. 
Pokhrel, D., Viraraghavan, T., 2004. Treatment of pulp and paper mill wastewater-a review. Science of the total environment 333, 37-58.

Polat, D., Balcı, İ., Özbelge, T.A., 2015. Catalytic ozonation of an industrial textile wastewater in a heterogeneous continuous reactor. Journal of Environmental Chemical Engineering 3, 1860-1871.

Poonkuzhali, K., Manivannan, M., Palvannan, T., 2013. Assessing the chelating ability of Aerva Lanata: Adsorption of chromium from tannery effluent and its toxicity measurement. Journal of Water Chemistry and Technology 35, 133-138.

Poonkuzhali, K., Sathishkumar, P., Boopathy, R., Palvannan, T., 2011a. Aqueous state laccase thermostabilization using carbohydrate polymers: Effect on toxicity assessment of azo dye. Carbohydrate Polymers 85, 341-348.

Poonkuzhali, K., Sathishkumar, P., Boopathy, R., Palvannan, T., 2011b. Aqueous state laccase thermostabilization using carbohydrate polymers: Effect on toxicity assessment of azo dye. Carbohydrate Polymers 85, 341-348.

Porras Torres, M., Cardozo Lopez, J., Chaparro, T.R., 2013. Removal of organic matterand toxicity in an upflow immobilized biomass anaerobic reactor treating hospital wastewater: preliminary evaluation. Dyna 80, 124-130.

Prajitha, V., Thoppil, J.E., 2016. Genotoxic and antigenotoxic potential of the aqueous leaf extracts of Amaranthus spinosus Linn. using Allium cepa assay. South African Journal of Botany 102, 18-25.

Prasad, A.A., Satyanarayana, V., Rao, K.B., 2013. Biotransformation of Direct Blue 1 by a moderately halophilic bacterium Marinobacter sp. strain HBRA and toxicity assessment of degraded metabolites. Journal of Hazardous Materials 262, 674-684.

Prieto, M.A., Prieto, I., Vázquez, J.A., Ferreira, I.C.F.R., 2015. An environmental management industrial solution for the treatment and reuse of mussel wastewaters. Science of The Total Environment 538, 117-128.

Pulate, P., Tarar, J., 2014. Cytogenetic effects of tilt on root tip meristem of onion Allium cepa L. International Journal of Plant, Animal and Environmental Sciences 4, 53-57.

Pyatkova, S., Eslina, A., Kozmin, G., 2009. Toxicity assessment of natural waters from the Obninsk radioactive waste repository. Radioprotection 44, 291-294.

Qin, R., Jiao, Y., Zhang, S., Jiang, W., Liu, D., 2010. Effects of aluminum on nucleoli in root tip cells and selected physiological and biochemical characters in Allium cepa var. agrogarum L. BMC plant biology 10, 225.

Quilang, J.P., Guzman, M., Hitta-Catalan, M., Rubio, R.O., Jacinto, S.D., Santiago, E.C., 2008. Effects of polychlorinated biphenyls (pcbs) on root meristem cells of common onion (Allium cepa L.) and on early life stages of Zebrafish (Danio rerio). Philippine Journal of Science 137, 141-151.

Qureshi, K., Ahmad, M., Bhatti, I., Iqbal, M., Khan, A., 2015a. Cytotoxicity reduction of wastewater treated by advanced oxidation process. Chemistry International 1, 53-59.
Radić, S., Stipaničev, D., Vujčić, V., Rajčić, M.M., Širac, S., Pevalek-Kozlina, B., 2010. The evaluation of surface and wastewater genotoxicity using the Allium cepa test. Science of The Total Environment 408, 1228-1233.

Ragunathan, I., Panneerselvam, N., 2007. Antimutagenic potential of curcumin on chromosomal aberrations in Allium cepa. Journal of Zhejiang University Science B 8, 470-475.

Rainho, C.R.d., Kaezer, A., Aiub, C.A., Felzenszwalb, I., 2010. Ability of Allium cepa L. root tips and Tradescantia pallida var. purpurea in N-nitrosodiethylamine genotoxicity and mutagenicity evaluation. Anais da Academia Brasileira de Ciências 82, 925-932.

Rajeshwari, A., Kavitha, S., Alex, S.A., Kumar, D., Mukherjee, A., Chandrasekaran, N., Mukherjee, A., 2015. Cytotoxicity of aluminum oxide nanoparticles on Allium cepa root tipeffects of oxidative stress generation and biouptake. Environmental Science and Pollution Research 22, 11057-11066.

Ramos de Rainho, C., Machado Corrêa, S., Luiz Mazzei, J., Alessandra Fortes Aiub, C., Felzenszwalb, I., 2013. Genotoxicity of polycyclic aromatic hydrocarbons and nitro-derived in respirable airborne particulate matter collected from urban areas of Rio de Janeiro (Brazil). BioMed research international 2013, http://dx.doi.org/10.1155/2013/765352.

Rank, J., 2003. The method of Allium anaphase-telophase chromosome aberration assay. Ekologija 1, 38-42.

Rank, J., Lopez, L., Nielsen, M., Moretton, J., 2002. Genotoxicity of maleic hydrazide, acridine and DEHP in Allium cepa root cells performed by two different laboratories. Hereditas 136, 13-18.

Rank, J., Nielsen, M., 1994. Evaluation of the Allium anaphasetelophase test in relation to genotoxicity screening of industrial wastewater. Mutation Research/Environmental Mutagenesis and Related Subjects 312, 17-24.

Rank, J., Nielsen, M.H., 1993. A modified Allium test as a tool in the screening of the genotoxicity of complex mixtures. Hereditas 118, 49-53.

Rank, J., Nielsen, M.H., 1997a. Allium cepa anaphasetelophase root tip chromosome aberration assay on $\mathrm{N}$ methyl-N-nitrosourea, maleic hydrazide, sodium azide, and ethyl methanesulfonate. Mutation Research/Genetic Toxicology and Environmental Mutagenesis 390, 121127.

Rank, J., Nielsen, M.H., 1997b. Allium cepa anaphasetelophase root tip chromosome aberration assay on $\mathrm{N}$ methyl-N-nitrosourea, maleic hydrazide, sodium azide, and ethyl methanesulfonate. Mutation Research/Genetic Toxicology and Environmental Mutagenesis 390, 121127.

Rank, J., Nielsen, M.H., 1998. Genotoxicity testing of wastewater sludge using the Allium cepa anaphasetelophase chromosome aberration assay. Mutation Research/Genetic Toxicology and Environmental Mutagenesis 418, 113-119. 
Rashid, A., Bhatti, H.N., Iqbal, M., Noreen, S., 2016. Fungal biomass composite with bentonite efficiency for nickel and zinc adsorption: a mechanistic study. Ecological engineering 91, 459-471.

Rathore, H.S., Choubey, P., 2005. Prevention of Acetaminophen-Induced Mitodepression with Myrobalan (Fruit of Terminalia chebula) in Allium cepa Model. Iranian Journal of Pharmacology \& Therapeutics 4, 100 104.

Ravindran, B., Kumari, S.S., Stenstrom, T., Bux, F., 2015. Evaluation of phytotoxicity effect on selected crops using treated and untreated wastewater from different configurative domestic wastewater plants. Environmental technology, 1-26.

Rencüzoğullari, E., Kayraldiz, A., Ila, H.B., Çakmak, T., Topaktaş, M., 2001. The cytogenetic effects of sodium metabisulfite, a food preservative in root tip cells of Allium cepa L. Turkish Journal of Biology 25, 361-370.

Renjana, P.K., Thoppil, J.E., 2015. Toxicological evaluation of root methanolic extract of strobilanthes heyneanus nees using Allium Test. International Journal of Pharmaceutical Sciences and Drug Research 5, 125-128.

Roa, O., Yeber, M., Venegas, W., 2012. Genotoxicity and toxicity evaluations of ECF cellulose bleaching effluents using the Allium cepa L. Test. Brazilian Journal of Biology 72, 471-477.

Rodrigues, F.P., Angeli, J.P.F., Mantovani, M.S., Guedes, C.L.B., JordÃ£o, B.Q., 2010. Genotoxic evaluation of an industrial effluent from an oil refinery using plant and animal bioassays. Genetics and Molecular Biology 33, 169-175.

Rodríguez, Y.A., Christofoletti, C.A., Pedro, J., Bueno, O.C., Malaspina, O., Ferreira, R.A.C., Fontanetti, C.S., 2015. Allium cepa and Tradescantia pallida bioassays to evaluate effects of the insecticide imidacloprid. Chemosphere 120, 438-442.

Roig, N., Sierra, J., Nadal, M., Martí, E., Navalón-Madrigal, P., Schuhmacher, M., Domingo, J.L., 2012. Relationship between pollutant content and ecotoxicity of sewage sludges from Spanish wastewater treatment plants. Science of The Total Environment 425, 99-109.

Ronchi, V.N., Bonatti, S., Turchi, G., 1986. Preferential localization of chemically induced breaks in heterochromatic regions of Vicia faba and Allium cepa chromosomes-I. Exogenous thymidine enhances the cytologic effects of 4-epoxyethyl-1,2-epoxy-cyclohexane. Environmental and Experimental Botany 26, 115-126.

Roy, A.K., Sharma, A., Talukder, G., 1989. A time-course study on effects of aluminium on mitotic cell division in Allium sativum. Mutation Research Letters 227, 221-226.

Roy, S., Nagarchi, L., Das, I., Mangalam Achuthananthan, J., Krishnamurthy, S., 2015. Cytotoxicity, Genotoxicity, and Phytotoxicity of Tannery Effluent Discharged into Palar River Basin, Tamil Nadu, India. Journal of toxicology 2015.

Roychoudhury, A., Giri, A.K., 1989. Effects of certain food dyes on chromosomes of Allium cepa. Mutation Research/Genetic Toxicology 223, 313-319.
Saeed, M., Adeel, S., Ilyas, M., Shahzad, M.A., Usman, M., Haq, E.-u., Hamayun, M., 2016. Oxidative degradation of Methyl Orange catalyzed by lab prepared nickel hydroxide in aqueous medium. Desalination and Water Treatment 57, 12804-12813.

Saghirzadeh, M., Gharaati, M.R., Mohammadi, S., GhiassiNejad, M., 2008. Evaluation of DNA damage in the root cells of Allium cepa seeds growing in soil of high background radiation areas of Ramsar - Iran. Journal of Environmental Radioactivity 99, 1698-1702.

Saleh Alqasoumi, T.H.K., Mohammed Al-Yahya, Ibrahim AlMofleh and Syed Rafatullah, 2012. Effect of Acute and Chronic Treatment of Common Spices in Swiss Albino Mice: A Safety Assessment Study. International Journal of Pharmacology 8, 80-90.

Salles, F.J., de Toledo, M.C.B., César, A.C.G., Ferreira, G.M., Barbério, A., 2016. Cytotoxic and genotoxic assessment of surface water from São Paulo State, Brazil, during the rainy and dry seasons. Ecotoxicology 25, 633-645.

Samuel, O.B., Osuala, F.I., Odeigah, P.G.C., 2010. Cytogenotoxicity evaluation of two industrial effluents using Allium cepa assay. African Journal of Environmental Science and Technology 4, 21-27.

Santana, G., Deus, M., Sousa, J., Ferreira, P., Fernandes, H., Peron, A., 2016. Antimitotic and antimutagenic action of the Hymenaea stigonocarpa bark on dividing cells. Brazilian Journal of Biology, http://dx.doi.org/10.1590/1519-6984.23014.

Sarkar, A.K., Ghorai, S., Patra, A.S., Mishra, B.K., Mandre, N.R., Pal, S., 2015. Modified amylopectin based flocculant for the treatment of synthetic effluent and industrial wastewaters. International Journal of Biological Macromolecules 72, 356-363.

Sarojini, S., Manimegala, G., Prakash, M., Ananthakrishnasamy, S., Gunasekaran, G., Karmegam, N., 2010. Vermicomposting of lignite flyash reduces its genotoxicity and phytotoxicity and improves its agronomic values for application as soil amendments in farmlands: a study of vermicomposted flyash on onion crops (Allium cepa). Dynamic Soil, Dynamic Plant 4, 135138.

Sasmaz, A., 2008. Translocation and accumulation of boron in roots and shoots of plants grown in soils of low boron concentration in Turkey's Keban $\mathrm{Pb}-\mathrm{Zn}$ mining area. International Journal of Phytoremediation 10, 302-310.

Sasmaz, A., 2009. The distribution and accumulation of selenium in roots and shoots of plants naturally grown in the soils of Keban's Pb-Zn-F mining area, Turkey. International Journal of Phytoremediation 11, 385-395.

Sasmaz, A., Dogan, I.M., Sasmaz, M., 2016a. Removal of Cr, Ni and Co in the water of chromium mining areas by using Lemna gibba L. and Lemna minor L. Water and Environment Journal 30, 235-242.

Sasmaz, A., Obek, E., 2009. The accumulation of arsenic, uranium, and boron in Lemna gibba L. exposed to secondary effluents. Ecological Engineering 35, 15641567. 
Sasmaz, A., Obek, E., 2012. The accumulation of silver and gold in Lemna gibba L. exposed to secondary effluents. Chemie der Erde-Geochemistry 72, 149-152.

Sasmaz, A., Obek, E., Hasar, H., 2008. The accumulation of heavy metals in Typha latifolia L. grown in a stream carrying secondary effluent. Ecological Engineering 33, 278-284.

Sasmaz, A., Sasmaz, M., 2009. The phytoremediation potential for strontium of indigenous plants growing in a mining area. Environmental and Experimental Botany 67, 139144.

Sasmaz, A., Yaman, M., 2008. Determination of uranium and thorium in soil and plant parts around abandoned leadzinc-copper mining area. Communications in Soil Science and Plant Analysis 39, 2568-2583.

Sasmaz, M., Akgül, B., Sasmaz, A., 2015a. Distribution and accumulation of selenium in wild plants growing naturally in the Gumuskoy (Kutahya) Mining Area, Turkey. Bulletin of Environmental Contamination and Toxicology 94, 598-603.

Sasmaz, M., Akgul, B., Yıldırım, D., Sasmaz, A., 2016 b. Bioaccumulation of thallium by the wild plants grown in soils of mining area. International Journal of Phytoremediation 18, 1164-1170.

Sasmaz, M., Akgül, B., Yıldırım, D., Sasmaz, A., 2016c. Mercury uptake and phytotoxicity in terrestrial plants grown naturally in the Gumuskoy (Kutahya) mining area, Turkey. International Journal of Phytoremediation 18, 69-76.

Sasmaz, M., Obek, E., Sasmaz, A., 2016d. Bioaccumulation of Uranium and Thorium by Lemna minor and Lemna gibba in $\mathrm{Pb}-\mathrm{Zn}-\mathrm{Ag}$ Tailing Water. Bulletin of Environmental Contamination and Toxicology 97, 832-837.

Sasmaz, M., Topal, E.I.A., Obek, E., Sasmaz, A., 2015b. The potential of Lemna gibba $\mathrm{L}$. and Lemna minor $\mathrm{L}$. to remove $\mathrm{Cu}, \mathrm{Pb}, \mathrm{Zn}$, and $\mathrm{As}$ in gallery water in a mining area in Keban, Turkey. Journal of Environmental Management 163, 246-253.

Şaşmaz, S., Gedikli, S., Aytar, P., Güngörmedi, G., Çabuk, A., Hür, E., Ünal, A., Kolankaya, N., 2011. Decolorization potential of some reactive dyes with crude laccase and laccase-mediated system. Applied Biochemistry and Biotechnology 163, 346-361.

Satar, R., Husain, Q., 2011. Catalyzed degradation of disperse dyes by calcium alginate-pectin entrapped bitter gourd (Momordica charantia) peroxidase. Journal of Environmental Sciences 23, 1135-1142.

Saxena, P., Chauhan, L., Chandra, S., Gupta, S., 2004. Genotoxic effects of diuron contaminated soil on the root meristem cells of Allium sativum: a possible mechanism of chromosome damage. Toxicology Mechanisms and Methods 14, 281-286.

Saxena, P., Chauhan, L., Gupta, S., 2005. Cytogenetic effects of commercial formulation of cypermethrin in root meristem cells of Allium sativum: Spectroscopic basis of chromosome damage. Toxicology 216, 244-252.
Saxena, P.N., Gupta, S.K., Murthy, R.C., 2010. Carbofuran induced cytogenetic effects in root meristem cells of Allium cepa and Allium sativum: A spectroscopic approach for chromosome damage. Pesticide Biochemistry and Physiology 96, 93-100.

Sayed, M., 2015. Efficient removal of phenol from aqueous solution by the pulsed high-voltage discharge process in the presence of $\mathrm{H}_{2} \mathrm{O}_{2}$. Chemistry International 1, 81-86.

Sayles, G.D., Acheson, C.M., Kupferle, M.J., Shan, Y., Zhou, Q., Meier, J.R., Chang, L., Brenner, R.C., 1999. Land treatment of PAH-contaminated soil: performance measured by chemical and toxicity assays. Environmental science \& Technology 33, 4310-4317.

Seow, W.Y., Hauser, C.A.E., 2016. Freeze-dried agarose gels: A cheap, simple and recyclable adsorbent for the purification of methylene blue from industrial wastewater. Journal of Environmental Chemical Engineering 4, 1714-1721.

Serra de Lima Moraes, D., Jordao, B.Q., 2001. Evaluation of the genotoxic potential of municipal waste water discharged into the Paraguay river during periods of flood and drought. Environmental Toxicology 16, 113-116.

Seth, C., Misra, V., Chauhan, L., Singh, R., 2008a. Genotoxicity of cadmium on root meristem cells of Allium cepa: cytogenetic and Comet assay approach. Ecotoxicology and Environmental Safety 71, 711-716.

Shaikh, S., Nazam, N., Lone, M.I., Ahmad, W., 2012. Dichlorophen and Dichlorovos mediated genotoxic and cytotoxic assessment on root meristem cells of Allium cepa. Science Diliman 24, 10.

Sharma, A., Kumar, M., Kaur, S., 2011. Cuminum cyminum Linn. and Coriandrum sativum Linn. extracts modulate chromium genotoxicity in Allium cepa chromosomal aberration assay. The Nucleus 54, 99-105.

Sharma, S., Nagpal, A., Vig, A.P., 2012. Genoprotective potential of Brassica juncea (L.) Czern. against mercuryinduced genotoxicity in Allium cepa L. Turkish Journal of Biology 36, 622-629.

Shashank, B., Suresh, D., 2013. Toxicity effects of municipal sewage effluent on onion roots. Journal of Environmental Research and Development 7, 1547.

Shibata, F., Hizume, M., 2002. The identification and analysis of the sequences that allow the detection of Allium cepa chromosomes by GISH in the allodiploid A. wakegi. Chromosoma 111, 184-191.

Shimizu, N., Itoh, N., Utiyama, H., Wahl, G.M., 1998. Selective entrapment of extrachromosomally amplified DNA by nuclear budding and micronucleation during $\mathrm{S}$ phase. The Journal of Cell Biology 140, 1307-1320.

Shimizu, N., Shimura, T., Tanaka, T., 2000. Selective elimination of acentric double minutes from cancer cells through the extrusion of micronuclei. Mutation Research/Fundamental and Molecular Mechanisms of Mutagenesis 448, 81-90.

Shindy, H., 2016. Basics in colors, dyes and pigments chemistry: A review. Chemistry International 2, 29-36. 
Shoukat, S., Bhatti, H.N., Iqbal, M., Noreen, S., 2017. Mango stone biocomposite preparation and application for crystal violet adsorption: A mechanistic study. Microporous and Mesoporous Materials 239, 180-189.

Siddiqui, A.H., Tabrez, S., Ahmad, M., 2011. Validation of plant based bioassays for the toxicity testing of Indian waters. Environmental Monitoring and Assessment 179, 241253.

Sik, L., Acar, O., Aki, C., 2009. Genotoxic effects of industrial wastewater on Allium cepa L. African Journal of Biotechnology 8, 5.

Silva, D.S., Barboza, B., Garcia, A.C., Oliveira, B.d., Estevam, C.S., A Neto, V., Santos, A.L., Dias, A.S., Scher, R., Pantaleao, S.M., 2013. Investigation of protective effects of Erythrina velutina extract against MMS induced damages in the root meristem cells of Allium cepa. Revista Brasileira de Farmacognosia 23, 273-278.

Silva, G.H.d., 2014. Chronic effects of silica nanoparticles in Vibrio fischeri, Raphidocelis subcaptata, Danio rerio and Allium cepa, Centro de Energia Nuclear na Agricultura. Universidade de São Paulo 10.11606/D.64.2014.tde12122014-100850.

Silveira, M.A.D., Ribeiro, D.L., dos Santos, T.A., Vieira, G.M., Cechinato, C.N., Kazanovski, M., d'Arce, L.P.G., 2015. Mutagenicity of two herbicides widely used on soybean crops by the Allium cepa test. Cytotechnology 68, 1-8.

Singh, M., Das, A., Singh, D., Maiti, P., Shabbir, M., Das, A., 2014. High genotoxicity of shipyard contaminants on Allium cepa and calf thymus DNA. Environmental Chemistry Letters 12, 321-327.

Singh, P., 2015. Toxic effect of Chromium on genotoxicity and Cytotoxicity by use of Allium Cepa L. International Journal of Research in Engineering and Applied Sciences 5, 1-10.

Singh, V., Tiwari, A., Das, M., 2016. Phyco-remediation of industrial waste-water and flue gases with algal-diesel engenderment from micro-algae: A review. Fuel 173, 9097.

Sinha, V.S., Kumar, N., 2014. Assessment of Mito-inhibitory and Genotoxic effects of two Organophosphate Pesticides in the root tip cells of Allium cepa L. Annals of Plant Sciences 3, 699-703.

Skipperud, L., Salbu, B., Hageb $\AA^{3}$, E., 1998. Speciation of trace elements in discharges from the pulp industry. The Science of the Total Environment 217, 251-256.

Ślusarczyk, J., Dudek, M., Wierzbicka, M., Suchocki, P., Kuraś, M., 2014. Antimitotic effect of Selol and sodium selenate (IV) on Allium test cells. Caryologia 67, 250-259.

Ślusarczyk, J., Wierzbicka, M., Suchocki, P., Kuraś, M., 2016. Ultrastructural changes in onion (Allium cepa L.) root tip meristem cells treated with Selol and sodium selenate (IV). Caryologia 68, 1-11.

Smaka-Kincl, V., Stegnar, P., Lovka, M., Toman, M.J., 1996. The evaluation of waste, surface and ground water quality using the Allium test procedure. Mutation Research/Genetic Toxicology 368, 171-179.
Sobral, O., Marin-Morales, M.A., Ribeiro, R., 2013. Could contaminant induced mutations lead to a genetic diversity overestimation? Ecotoxicology 22, 838-846.

Solanke, P., 2008. An Evaluation of the Genotoxic Effects of the Seed Decoction of Cassia tora Linn.(Leguminosae) in an Allium cepa Model. Ethnobotanical Leaflets 2008, 125.

Soliman, M.I., 2001. Genotoxicity testing of neem plant (Azadirachta indica A. Juss.) using the Allium cepa chromosome aberration assay. Journal of Biological Sciences 1, 1021-1027.

Somashekar, R., Arekal, G.D., 1983. Chromosomal aberrations induced by electroplating waste water. Cytologia 48, 621-625.

Sondhi, N., Bhardwaj, R., Kaur, S., Kumar, N., Singh, B., 2008. Isolation of 24-epibrassinolide from leaves of Aegle marmelos and evaluation of its antigenotoxicity employing Allium cepa chromosomal aberration assay. Plant Growth Regulation 54, 217-224.

Soodan, R.K., Katnoria, J.K., Nagpal, A., 2014. Allium cepa Root Chromosomal Aberration Assay: An Efficient Test System for Evaluating Genotoxicity of Agricultural Soil. International Journal of Science and Research 3, 245-250.

Souza, T., Hencklein, F., Angelis, D., GonÃßalves, R., Fontanetti, C., 2009. The Allium cepa bioassay to evaluate landfarming soil, before and after the addition of rice hulls to accelerate organic pollutants biodegradation. Ecotoxicology and Environmental Safety 72, 1363-1368.

Sridhar, M., Ana, G., Wai-Ogosu, O., 2009. Characteristics of Effluent from a Chemical Fertilizer Industry in Southern Nigeria. Journal of Applied Science, Engineering and Technology http://dx.doi.org/10.4314/jaset.v7i1.42723.

Srivastava, K., Mishra, K.K., 2009. Cytogenetic effects of commercially formulated atrazine on the somatic cells of Allium cepa and Vicia faba. Pesticide Biochemistry and Physiology 93, 8-12.

Srivastava, R., Kumar, D., Gupta, S., 2005. Bioremediation of municipal sludge by vermitechnology and toxicity assessment by Allium cepa. Bioresource Technology 96, 1867-1871.

Staykova, T.A., Ivanova, E.N., Velcheva, I.G., 2005. Cytogenetic effect of heavy-metal and cyanide in contaminated waters from the region of southwest Bulgaria. Journal of Cell and Molecular Biology 4, 41-46.

Sudhakar, R., Ninge Gowda, K., Venu, G., 2001. Mitotic abnormalities induced by silk dyeing industry effluents in the cells of Allium cepa. Cytologia 66, 235-240.

Sueviriyapan, N., Suriyapraphadilok, U., Siemanond, K., Quaglia, A., Gani, R., 2016. Industrial wastewater treatment network based on recycling and rerouting strategies for retrofit design schemes. Journal of Cleaner Production 111, Part A, 231-252.

Sultan, A.Ö., Çelik, T.A., 2009. Genotoxic and antimutagenic effects of Capparis spinosa L. on the Allium cepa L. root tip meristem cells. Caryologia 62, 114-123.

Suryavathi, V., Sharma, S., Saxena, P., Pandey, S., Grover, R., Kumar, S., Sharma, K., 2005. Acute toxicity of textile dye 
wastewaters (untreated and treated) of Sanganer on male reproductive systems of albino rats and mice. Reproductive Toxicology 19, 547-556.

Tabet, M., Abda, A., Benouareth, D.E., Liman, R., Konuk, M., Khallef, M., Taher, A., 2015. Mutagenic and genotoxic effects of Guelma's urban wastewater, Algeria. Environmental Monitoring and Assessment 187, 1-13.

Tabrez, S., Ahmad, M., 2011. Oxidative stress-mediated genotoxicity of wastewaters collected from two different stations in northern India. Mutation Research/Genetic Toxicology and Environmental Mutagenesis 726, 15-20.

Tabrez, S., Ahmad, M., 2012. Genotoxicity of trichloroethylene in the natural milieu. International Journal of Hygiene and Environmental Health 215, 333-338.

Tahir, M.A., Bhatti, H.N., Iqbal, M., 2016a. Solar Red and Brittle Blue direct dyes adsorption onto Eucalyptus angophoroides bark: Equilibrium, kinetics and thermodynamic studies. Journal of Environmental Chemical Engineering 4, 2431-2439.

Tahir, N., Bhatti, H.N., Iqbal, M., Noreen, S., 2016b. Biopolymers composites with peanut hull waste biomass and application for Crystal Violet adsorption. International Journal of Biological Macromolecules 94, 210-220.

Tamis, J., Lužkov, K., Jiang, Y., Loosdrecht, M.C.M.v., Kleerebezem, R., 2014. Enrichment of Plasticicumulans acidivorans at pilot-scale for PHA production on industrial wastewater. Journal of Biotechnology 192, Part A, 161-169.

Tanti, B., Buragohain, A.K., Dutta, S., Gurung, L., Shastry, M., Borah, S.P., 2009. Studies on the cytotoxic effect of oil refinery sludge on root meristem. Advances in Environmental Biology 3, 10-15.

Tartar, G., Kaymak, F., Muranli, F.D.G., 2006. Genotoxic Effects of Avenoxan on Allium cepa L. and Allium sativum L. Caryologia 59, 241-247.

Teena, M., Soumya, K., Sudha, K., 2016. Cytotoxic effect of sewage effluent on root tip cells of Allium cepa L. South Indian Journal Of Biological Sciences 2, 18-23.

Teerarak, M., Bhinija, K., Thitavasanta, S., Laosinwattana, C., 2009. The impact of sodium chloride on root growth, cell division, and interphase silver-stained nucleolar organizer regions (AgNORs) in root tip cells of Allium cepa L. Scientia Horticulturae 121, 228-232.

Thakkar, R.R., Wang, O.L., Zerouga, M., Stillwell, W., Haq, A., Kissling, R., Pierce, W.M., Smith, N.B., Miller, F.N., Ehringer, W.D., 2000. Docosahexaenoic acid reverses cyclosporin A-induced changes in membrane structure and function. Biochimica et Biophysica Acta (BBA)General Subjects 1474, 183-195.

Timothy, O., Idu, M., Olorunfemi, D.I., Ovuakporie-Uvo, O., 2014. Cytotoxic and genotoxic properties of leaf extract of Icacina trichantha Oliv. South African Journal of Botany 91, 71-74.

Tipirdamaz, R., Gomurgen, A.N., Kolankaya, D., Dogan, M., 2003. Determination of toxicity of pulp-miii effluents by using Allium test. Tarim Bilimleri Dergisi 9, 93-97.
Tkalec, M., Malarić, K., Pavlica, M., Pevalek-Kozlina, B., Vidaković-Cifrek, Ž., 2009. Effects of radiofrequency electromagnetic fields on seed germination and root meristematic cells of Allium cepa L. Mutation Research/Genetic Toxicology and Environmental Mutagenesis 672, 76-81.

Torres, M.P., López, J.C., Chaparro, T.R., 2014. Removal of organic matter and toxicity in an upflow immobilized biomass anaerobic reactor treating hospital wastewater: preliminary evaluation. Dyna 80, 124-130.

Tripathy, S., Bijayinee, S., Samad, I., Das, R., 2013. Endosulfan: A potential genotoxicant on Allium cepa root tip cells. Journal of Agricultural Biotechnology and Sustainable Development 5, 29.

Tripathy, S., Rao, D., 2015. Mitotic aberrations induced by orange red (a food additive dye) as a potential genotoxicant on root tip cells of onion (Allium cepa L.). International Food Research Journal 22, 383-392.

Tripathy, S.K., Patel, S., 2015. Abnormal mitosis in root meristem cells of Allium cepa L. induced by a fabric dye reactive turquoise blue (Procion MX). African Journal of Biotechnology 13, 15-20.

Trushin, M.V., Ardashov, O.V., Volcho, K.P., Arkharova, I.A., Salakhutdinov, N.F., 2013a. Genotoxicological Safety Assessment of a New Antiparkinsonian Substance ( $(1 \mathrm{r}$, 2r, 6s)-3-methyl-6-(Prop-1-en-2-yl) cyclohex-3-ene-1, 2diol). World Journal of Medical Sciences 8, 355-358.

Trushin, M.V., Ratushnyak, A.Y., Arkharova, I.A., Ratushnyak, A.A., 2013b. Genetic alterations revealed in Allium cepatest system under the action of some xenobiotics. World Applied Sciences Journal 22, 342-344.

Turkmen, Z., Cavusoglu, K., Yapar, K., Yalcin, E., 2009. Protective role of Royal Jelly (honeybee) on genotoxicity and lipid peroxidation, induced by petroleum wastewater, in Allium cepa L. root tips. Environmental Technology 30, 1205-1214.

Turkoglu, S., 2007. Genotoxicity of five food preservatives tested on root tips of Allium cepa L. Mutation Research/Genetic Toxicology and Environmental Mutagenesis 626, 4-14.

Türkoğlu, Ş., 2008. Evaluation of genotoxic effects of sodium propionate, calcium propionate and potassium propionate on the root meristem cells of Allium cepa. Food and Chemical Toxicology 46, 2035-2041.

Türkoğlu, Ş., 2012. Determination of genotoxic effects of chlorfenvinphos and fenbuconazole in Allium cepa root cells by mitotic activity, chromosome aberration, DNA content, and comet assay. Pesticide Biochemistry and Physiology 103, 224-230.

Udalova, A.A., Geras'kin, S.A., Dikarev, V.G., Dikareva, N.S., 2014. Assessment of cyto- and genotoxicity of natural waters in the vicinity of radioactive waste storage facility using Allium-test. Radiatsionnaia Biologiia, Radioecologiia 54, 97-106.

Ukaegbu, M.C., Odeigah, G., 2009. The genotoxic effect sewage effluent on Allium cepa. Report and Opinion 1, 36-41. 
Ukpaka, C., 2016a. BTX Degradation: The concept of microbial integration. Chem Int 3, 8-18.

Ukpaka, C., 2016b. Development of model for bioremediation of crude oil using moringa extract. Chemistry International 2, 19-28.

Ukpaka, C., 2016c. Empirical model approach for the evaluation of $\mathrm{pH}$ and conductivity on pollutant diffusion in soil environment. Chemistry International 2, 267-278.

Ukpaka, C., 2016d. Predictive model on the effect of restrictor on transfer function parameters on pneumatic control system. Chemistry International 2, 128-135.

Ukpaka, C., Wami, E., Amadi, S., 2015. Effect of pollution on metal corrosion: A case study of carbon steel metal in acidic media. Current Science Perspectives 1, 107-111.

Ukpaka, C.P., 2016e. BTX Degradation: The concept of microbial integration. Chemistry International 3, 8-18.

Ukpaka, C.P., 2016f. Empirical model approach for the evaluation of $\mathrm{pH}$ and conductivity on pollutant diffusion in soil environment. Chemistry International 2, 267-278.

Ukpaka, C.P., 2016g. Predictive model on the effect of restrictor on transfer function parameters on pneumatic control system. Chemistry International 2, 128-135.

Ukpaka, C.P., Collins, I., 2016. Characteristics concept for the examination of total hydrocarbon content and total organic carbon in contaminated soil zone. Current Science Perspectives 2, 69-77.

Amadi, S.A ., Ukpaka C.P., 2016h. Role of molecular diffusion in the recovery of water flood residual oil. Chemistry International 2, 103-114.

Ullah, I., Nadeem, R., Iqbal, M., Manzoor, Q., 2013. Biosorption of chromium onto native and immobilized sugarcane bagasse waste biomass. Ecological Engineering 60, 99107.

Umebese, C., Azeez, T., Adekoya, K., 2013. Role of ethylenediamine tetraacetic acid and salicylic acid in alleviating cytogenetic toxicity of copper in roots of Allium cepa (L.). Genetics and Plant Physiology 3, 98-108.

Vasiliadou, I.A., Pariente, M.I., Martinez, F., Melero, J.A., Molina, R., 2016. Modeling the integrated heterogeneous catalytic fixed-bed reactor and rotating biological contactor system for the treatment of poorly biodegradable industrial agrochemical wastewater. Journal of Environmental Chemical Engineering 4, 23132321.

Ventura-Camargo, B., Maltempi, P., Marin-Morales, M., 2011. The use of the cytogenetic to identify mechanisms of action of an azo dye in Allium cepa meristematic cells. Journal of Environmental and Analytical Toxicology 1, 2161-0525.1000109.

Viana, L.Á., Düsman, E., Vicentini, V.E.P., 2014. Mutagenicity and cytotoxicity of liquid waste, press water and pond water, produced in the cassava flour industry, and of antitoxic sodium thiosulfate. Journal of the Science of Food and Agriculture 94, 453-458.

Vianna Villela, I., Marques De Oliveira, I., Coelho Silveira, J., Ferraz Dias, J., Pegas Henriques, J.A., Da Silva, J., 2007. Assessment of environmental stress by the micronucleus and comet assays on Limnoperna fortunei exposed to GuaÃba hydrographic region samples (Brazil) under laboratory conditions. Mutation research. Genetic Toxicology and Environmental Mutagenesis 628, 76-86.

Vidaković, Ž., Papeš, D., Tomić, M., 1993. Toxicity of waste drilling fluids in modified Allium test. Water, air, and soil pollution 69 , 413-423.

Vujošević, M., Anđelković, S., Savić, G., Blagojević, J., 2008. Genotoxicity screening of the river Rasina in Serbia using the Allium anaphase-telophase test. Environmental Monitoring and Assessment 147, 75-81.

Vujosevic, M., Blagojevic, J., Martinovic _Vitanovic, V., Kalafatic, V., 2001. The presence of genotoxic substances in the river Danube five months after bombardment of the industrial zone of Pancevo as revealed by the Allium anaphase-telophase genotoxicity assay. Acta veterinaria 51, 373-382.

Vymazal, J., 2014. Constructed wetlands for treatment of industrial wastewaters: A review. Ecological Engineering 73, 724-751.

Wang, A.-J., Wang, L.-P., 2007. The study on induced effect of detergent by the micronucleus test of Allium cepa root tips. Journal of Hebei North University (Natural Science Edition) 2, 007.

Wängberg, S., Bergström, B., Blanck, H., Svanberg, O., 1995. The relative sensitivity and sensitivity patterns of shortterm toxicity tests applied to industrial wastewaters. Environmental Toxicology and Water Quality 10, 81-90.

Wardini, T.H., Notodarmojo, P.A., 2015. TPK Sarimukti, Cipatat, West Bandung compost toxicity test using Allium test, the $5^{\text {th }}$ international conference on mathematics and natural sciences. aip publishing, pp. 110006, http://dx.doi.org/110010.111063/110001.4930777.

Wasi, S., Tabrez, S., Ahmad, M., 2011. Detoxification potential of Pseudomonas fluorescens SM1 strain for remediation of major toxicants in Indian water bodies. Water, Air, \& Soil Pollution 222, 39-51.

Watharkar, A.D., Jadhav, J.P., 2014. Detoxification and decolorization of a simulated textile dye mixture by phytoremediation using Petunia grandiflora and, Gailardia grandiflora: a plant-plant consortial strategy. Ecotoxicology and Environmental Safety 103, 1-8.

Watharkar, A.D., Rane, N.R., Patil, S.M., Khandare, R.V., Jadhav, J.P., 2013. Enhanced phytotransformation of Navy Blue RX dye by Petunia grandiflora Juss. with augmentation of rhizospheric Bacillus pumilus strain PgJ and subsequent toxicity analysis. Bioresource Technology 142, 246-254.

White, P.A., Rasmussen, J.B., 1998. The genotoxic hazards of domestic wastes in surface waters1. Mutation Research/Reviews in Mutation Research 410, 223-236.

Whysner, J., Vijayaraj Reddy, M., Ross, P.M., Mohan, M., Lax, E.A., 2004. Genotoxicity of benzene and its metabolites. Mutation Research/Reviews in Mutation Research 566, 99-130.

Wierzbicka, M., 1994. Resumption of mitotic activity in Allium cepa L. root tips during treatment with lead salts. Environmental and Experimental Botany 34, 173-180. 
Wierzbicka, M., 1999a. Comparison of lead tolerance in Allium cepa with other plant species. Environmental Pollution 104, 41-52.

Wierzbicka, M., 1999b. The effect of lead on the cell cycle in the root meristem of Allium cepa L. Protoplasma 207, 186-194.

Wu, L., Yi, H., Yi, M., 2010. Assessment of arsenic toxicity using Allium/Vicia root tip micronucleus assays. Journal of Hazardous Materials 176, 952-956.

Xing, H., Liang, W., Hou, S., 1995. The affection of chemical industrial affluent on the nuclear structure of tip cells in Allium cepa. Acta Botanica Boreali-Occidentalia Sinica 16, 262-266.

Yahaya, T., Okpuzor, J., Esther, 0.0., 2012. Investigation of Cytotoxicity and Mutagenicity of Cement Dust Using Allium cepa Test. Research Journal of Mutagenesis 2, 1018.

Yekeen, T.A., Adeboye, M.K., 2013. Cytogenotoxic effects of cypermethrin, deltamethrin, lambdacyhalothrin and endosulfan pesticides on Allium cepa root cells. African Journal of Biotechnology 12, 6000.

Yen, N.T., Oanh, N., Reutergardh, L.B., Wise, D.L., Lan, N.T.T., 1996. An intergrated waste survey and environmental effects of COGIDO, a bleached pulp and paper mill in Vietnam, on the receiving waterbody. Resources, Conservation and Recycling 18, 161-173.

Yi, H., Meng, Z., 2003. Genotoxicity of hydrated sulfur dioxide on root tips of Allium sativum and Vicia faba. Mutation Research/Genetic Toxicology and Environmental Mutagenesis 537, 109-114.

Yi, H., Wu, L., Jiang, L., 2007a. Genotoxicity of arsenic evaluated by Allium-root micronucleus assay. Science of the total environment 383, 232-236.

Yi, H., Wu, L., Jiang, L., 2007b. Genotoxicity of arsenic evaluated by Allium-root micronucleus assay. Science of The Total Environment 383, 232-236.

Yıldız, M., Ciğerci, İ.H., Konuk, M., Fatih Fidan, A., Terzi, H., 2009. Determination of genotoxic effects of copper sulphate and cobalt chloride in Allium cepa root cells by chromosome aberration and comet assays. Chemosphere 75, 934-938.

Younas, A., Nadeem, N., Iqbal, M., 2015. Ag(I), Cr(VI) and $\mathrm{Pb}$ (II) adsorption from aqueous media onto different adsorbent types. Asian Journal of Chemistry 27, 33083314.

Younis, S.A., Al-Hakkak, Z.S., Al-Rawi, F.I., Hagop, E.G., 1989. Physiological and cytogenetic effects of phosphine gas in Allium cepa (L.). Journal of Stored Products Research 25, 25-30.

Yuet Ping, K., Darah, I., Yusuf, U.K., Yeng, C., Sasidharan, S., 2012. Genotoxicity of Euphorbia hirta: an Allium cepa assay. Molecules 17, 7782-7791.

Yüzbașioglu, D., scedil, ioglu, D., 2003. Cytogenetic effects of fungicide afugan on the meristematic cells of Allium cepa L. Cytologia 68, 237-243.
Zakharov, Y., Bondareva, L., 2015. Simulation of domestic and industrial wastewater disposal in flooded mine workings. Procedia Engineering 117, 389-396.

Zarpelon, F., Galiotto, D., Aguzzoli, C., Carli, L.N., Figueroa, C.A., Baumvol, I.J.R., Machado, G., Crespo, J.d.S., Giovanela, M., 2016. Removal of coliform bacteria from industrial wastewaters using polyelectrolytes/silver nanoparticles self-assembled thin films. Journal of Environmental Chemical Engineering 4, 137-146.

Zheng, X., Zhang, Z., Yu, D., Chen, X., Cheng, R., Min, S., Wang, J., Xiao, Q., Wang, J., 2015. Overview of membrane technology applications for industrial wastewater treatment in China to increase water supply. Resources, Conservation and Recycling 105, Part A, 1-10.

Visit us at: http://bosaljournals.com/chemint/ Submissions are accepted at: editorci@bosaljournals.com 